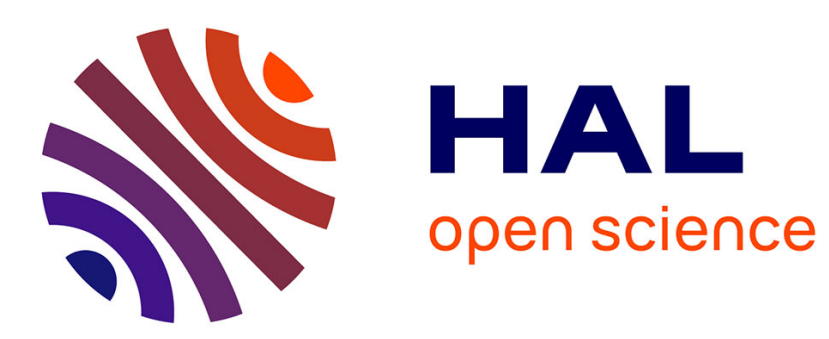

\title{
Mixed and hybrid finite element methods
}

Jean Roberts, Jean-Marie Thomas

\section{To cite this version:}

Jean Roberts, Jean-Marie Thomas. Mixed and hybrid finite element methods. [Research Report] RR-0737, INRIA. 1987. inria-00075815

\section{HAL Id: inria-00075815 https://hal.inria.fr/inria-00075815}

Submitted on 24 May 2006

HAL is a multi-disciplinary open access archive for the deposit and dissemination of scientific research documents, whether they are published or not. The documents may come from teaching and research institutions in France or abroad, or from public or private research centers.
L'archive ouverte pluridisciplinaire HAL, est destinée au dépôt et à la diffusion de documents scientifiques de niveau recherche, publiés ou non, émanant des établissements d'enseignement et de recherche français ou étrangers, des laboratoires publics ou privés. 


\section{Rapports de Recherche}

UNITÉ DE RECHERCHE INRIA-ROCQUENCOURT

$\mathbf{N}^{\circ} \mathbf{7 3 7}$

\section{MIXED AND HYBRID FINITE ELEMENT METHODS}

Institut National de Recherche en Informatique et en Automatique $+$

a)

Domaine de Voluceau Rocquencourt -

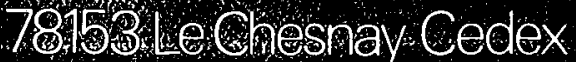
Firance

Jean E. ROBERTS Jean-Marie THOMAS 


\section{MIXED AND HYBRID METHODS}

$* * * * * * * * * * * * *$

\section{METHODES MIXTES ET HYBRIDES}

\section{$* * * * * * * * * * * * *$ \\ Jean E. ROBERTS ${ }^{1}$ - Jean-Marie THOMAS ${ }^{2}$}

1 - INRIA, B.P. 105, 78153 Le Chesnay Cédex

2 - Département de Mathématiques, Université de Pau, 64000 Pau

*This work will appear as a chapter in Handbook of Numerical Analysis vol.3 : Finite element methods, P.G. CIARLET \& J.L. LIONS editors, North Holland, Amsterdam. 


\begin{abstract}
This work, essentially expository in nature, is a survey of mixed and hybrid finite element methods and is meant to serve as a basic reference on the subject. Detailed descriptions of these methods including convergence results and error estimates are given for a second order elliptic problem in $\mathbb{R}^{n}$, for $n=2,3$. References for extensions of these methods to more complicated problems are given.
\end{abstract}

\title{
Rêsumé
}

Ce travail est un exposé des méthodes d'éléments finis mixtes et hybrides qui devrait servir de référence sur ce sujet. Des descriptions détaillées de ces méthodes, incluant des résultats de convergence et des estimations des erreurs, sont données pour un problème elliptique du deuxième ordre dans $\mathbb{I}^{n}$, pour $n=2,3$. On donne des références pour des extensions de ces méthodes à des problèmes plus compliqués.

Key words : mixed finite element methods, hybrid finite element methods

Mots-clés : méthodes d'éléments finis mixtes, méthodes d'éléments finis hybrides 


\section{CHAPTER I :}

\section{INTRODUCTION}

1 Preliminary remarks.

2 Conforming and equilibrium variational formulations for a model boundary value problem.

3 First examples of mixed and hybrid formulations.

4 General orientation.

\section{CHAPTER II :}

FINITE ELEMENT TYPE INTERPOLATION OF SCALAR AND VECTORIAL FUNCTIONS

5 Lagrangian interpolation ; an $\mathrm{H}^{1}$-approximation of scalar functions.

6 Interpolation of the moments of a vectorial function; an $H$ (div)-approximation of vector valued functions.

7 Examples of finite element type approximation for non triangular geometric forms.

\section{CHAPTER III :}

ABSTRACT THEORY OF APPROXIMATION OF SOLUTIONS OF PROBLEMS WITH

\section{LAGRANGIAN MULTIPLIERS}

8 General remarks

9 Analysis and approximation of the solution of a variational problem (theory of Babuska).

10 Analysis and approximation of the solution of a variational problem (theory of Brezzi).

11 Effects of numerical integration. 


\section{CHAPTER IV :}

MIXED FINTTE ELEMENT METHODS

12 Examples of primal mixed methods for a Dirichlet problem.

13 Examples of dual mixed methods for a Dirichlet problem.

14 Examples of mixed methods for a Neumann problem.

15 Examples of mixed finite element methods for a Robin's boundary condition.

16 Other examples of mixed finite element methods.

17 Solution of the linear system.

\section{CHAPTER V : \\ HYBRID FINITE ELEMENT METHODS}

18 Examples of primal hybrid finite element methods.

19 Primal hybrid methods and nonconforming methods.

20 Examples of dual hybrid finite element methods.

21 Hybridization of equilibrium methods.

\section{CHAPTER VI :}

\section{EXTENSIONS AND VARIATIONS}

22 Other examples of mixed and hybrid methods.

23 Extensions and variations of the theory. 


\section{CHA P TER I}

\section{INTRODUCTION}

\section{1 - PRELIMINARY REMARKS}

The terms "mixed finite element method", "hybrid finite element method", and even "mixed - hybrid finite element method" come essentially from the vocabulary of structural mechanics as do the terms "conforming finite element method" and "equilibrium finite element method" ; cf. in particular the historical account of hybrid and mixed elements given by PIAN (1983). Consider for simplicity the linear elasticity problem describing the displacement $\underline{u}$ of an elastic body $\Omega$. A finite element method based on the variational formulation associated with the principle of minimization of the potential energy form is called a displacement method as the procedure yields an approximation of the displacement field $u_{\sim}$. However, in structural mechanics one is often more interested in the stress tensor $\underset{\approx}{\sigma}$ than in the displacement. Methods have been devised for computing an approximation of $\underset{\approx}{\sigma}$ from the approximation of $\underset{\sim}{u}$. Alternatively the stress may be characterized as the minimum of the complementary energy form on the space of symmetric tensors satisfying a relation expressing the equilibrium between the internal and boundary forces acting on $\Omega$, and finite element methods based on the associated variational form are termed equilibrium methods. The drawback of these methods is that it is not usually easy to construct an approximation space of tensors satisfying the equilibrium relation and having the required amount of regularity, $C^{0}$ in this instance. Mixed methods and hybrid methods were devised to avoid this difficulty. 
With the aid of Lagrangian multipliers the above contrained minimization problem may be turned into a saddle point problem. The Hellinger-Reissner principle characterizes the pair $(\underset{\approx}{\sigma}, u)$ as the saddle point of a Lagrangian where $\sigma$ is allowed to vary over the space of symmetric tensors subject only to regularity contraints, the equilibrium relation now being incorporated in the Lagrangian. Finite element methods based on the corresponding variational principle are called mixed methods. Mixed methods were first introduced in the literature in HERMANN (1967) for the plate problem. We shall say, more generally, that a finite element method is a mixed method if it involves the simultaneous approximation of two or more vector fields defined on the physical domain. These will in general be the principal unknown and an expression involving one or more of its derivatives.

An alternative approach is taken in the development of hybrid methods. The idea is to retain the equilibrium requirement for functions in the space in which the stress is approximated but to relax the regularity requirement. Given a finite element mesh on the domain $\Omega$, a mesh dependent Lagrangian is constructed for which the unique saddle point is $(\underset{\approx}{\sigma}, \underline{\text { ) }}$ ) where $\underset{\approx}{\sigma}$ varies over a space of tensors satisfying the equilibrium condition but defined only on the union of the interiors of the elements. The Lagrangian multiplier $\phi$ is a vector field defined on the union of the boundaries of the elements which turns out to be none other than the trace of the displacement $\underline{u}$. Finite element methods based on the associated variational formulation are hybrid methods. It is equally possible, starting from the original displacement formulation, to define a Lagrangian, again mesh dependent, by introducing a multiplier $\lambda$ to relax the regularity requirement on the space in which the displacement $\underset{\sim}{u}$ is sought. The space of multipliers is again a space of vector fields on the union of the boundaries of the elements, and $u$ is looked for in a space of vector fields defined on the interiors of the elements. There is a unique saddle point $(\underline{u}, \lambda)$ for this Lagrangian and, morever, the multiplier $\lambda$ is the normal constraint $\underset{\sigma}{\sigma} \nu$ on the boundaries of the elements, $\nu$ denoting a field of unit normal vectors on the union of the boundaries. Methods obtained from such a Lagrangian are also termed hybrid methods. Again we shall adopt a general tcrminology saying that the method is a hybrid method if it involves the simultaneous approximation of a vector field defined on the union of the elements of the discretization and another defined on the union of the boundaries of the elements. The first reference for hybrid methods is JONES (1964).

An aperçu of these methods is given in CIARLET (1978, Chapter 7).

Besides structural mechanics mixed and hybrid methods have been found useful in many fields. In fluid mechanics for the incompressible stokes problem, for 
instance, the role of the displacement is played by the fluid pressure, that of the stress by the fluid velocity, that of the equilibrium condition by the incompressibility condition.

The above ideas will be developped in detail in Sections 2 and 3 in the context of a simpler model problem. First, however, we need to describe the functional framework in which we shall work.

Let $\Omega$ be a bounded, open subset of $R^{n}$, with $n=2$ or $n=3$, having a boundary $\Gamma$, $\Gamma=\partial \Omega$, which is piecewise $c^{1}$. For each natural number $m$ denote by $H^{m}(\Omega)$ the Sobolev space of order $m$ of scalar valued functions on $\Omega$, defined recursively by

$$
\left\{\begin{array}{c}
H^{0}(\Omega)=L^{2}(\Omega) ; \\
H^{m}(\Omega)=\left\{v \in H^{m-1}(\Omega) ; \forall \alpha=\left(\alpha_{1}, \ldots, \alpha_{n}\right) \in \mathbb{I N}^{n} \text { with } \sum_{i=1}^{n} \alpha_{i}=m\right. \\
\left.\partial^{\alpha} v=\frac{\partial^{m} v}{\partial x_{1} \alpha_{1} \ldots \partial x_{n}^{\alpha}} \in L^{2}(\Omega)\right\}
\end{array}\right.
$$

We put

$$
\begin{cases}\|v\|_{0, \Omega}=\left(\int_{\Omega} v^{2}(x) d x\right)^{3 / 2} \\ |v|_{m, \Omega}=\left(\sum_{|\alpha|=m}\left\|\partial^{a} v\right\|_{0, \Omega}^{2}\right)^{\frac{1}{2}} & \text { for all } m \geq 1 \\ \|v\|_{m, \Omega}=\left(\|v\|_{m-1, \Omega}^{2}+|v|_{m, \Omega}^{2}\right)^{3 / 2} & \text { for all } m \geq 1\end{cases}
$$

The product space

$$
\left(H^{m}(\Omega)\right)^{n}=\left\{\underline{q}=\left(q_{i}\right)_{1 \leq i \leq n} ; q_{i} \in H^{m}(\Omega) \text { for all } i=1, \ldots, n\right)
$$

will be equiped with the following seminorm and norm :

$$
\left\{\begin{array}{l}
|q|_{m, \Omega}=\left(\sum_{i=1}^{n}\left|q_{i}\right|_{m, \Omega}^{2}\right)^{\frac{1}{2}}, \\
\|q\|_{m, \Omega}=\left(\sum_{i=1}^{n}\left\|q_{i}\right\|_{m, \Omega}^{2}\right)^{3 / 2} .
\end{array}\right.
$$

We shall make use of the following result from functional analysis : 
THEOREM 1.1. The mapping $\mathrm{v} \longrightarrow \mathrm{V}_{\Gamma}$ defined a priori for functions $\mathrm{v}$ regular on $\bar{\Omega}$, can be extended to a continuous linear mapping called the trace map of $\mathrm{H}^{1}(\Omega)$ into $L^{2}(\Gamma)$. In other words there exists a constant $C$, depending only on $\Omega$, such that we have

$$
\|v\|_{0, \Gamma} \leq c\|v\|_{1, \Omega}
$$$$
\text { for all } \mathrm{v} \in \mathrm{H}^{1}(\Omega) \text {. }
$$

It can be shown that on the kernel $H_{0}^{1}(\Omega)$ of the trace mapping the correspondence $\mathrm{v} \longrightarrow>\left.\mathrm{v}\right|_{1, \Omega}$ defines a norm equivalent to the norm $\mathrm{v} \longrightarrow\|v\|_{1, \Omega}$; i.e. the Friedrichs-Poincaré inequality holds on $\mathrm{H}_{0}^{1}(\Omega)$. The image of the trace mapping, denoted $\mathrm{H}^{\frac{1 / 2}{2}}(\Gamma)$, is a Hilbert space with norm

$$
\|\psi\|_{\xi_{2}, \Gamma}=\inf _{\left\{v \in H^{1}(\Omega) ;\left.\right|_{\Gamma}=\psi\right\}}\|v\|_{1, \Omega}
$$

Thus for each vector $q=\left(q_{i}\right)_{1 \leq i \leq n} \in\left(H^{1}(\Omega)\right)^{n}$ the $n$ traces $\left.q_{i}\right|_{\Gamma}$ are defined and belong to $L^{2}(\Gamma)$, and in particular, the linear combination $q \cdot \underline{\nu}=\sum_{i=1}^{n} q_{\hat{1}}{ }_{\Gamma} \nu_{i}$, where $\underline{\nu}=\left(\nu_{i}\right)_{1 \leq i \leq n}$ denotes the unit exterior normal vector to $\Omega$, is in $L^{2}(\Gamma)$. Imposing less regularity on each component $q_{i}$, we can still define the normal trace as an element of a space of distributions on $\Gamma$, cf. Theorem 1.2 below. First, though, we need to introduce two more spaces. The dual space of $H^{3 / 2}(\Gamma)$ is denoted $H^{-\frac{1}{2}}(\Gamma)$ and is a Hilbert space with norm

$$
\|\mu\|_{-\frac{1}{2}, \Gamma}=\sup _{\left\{\psi \in H^{3 / 2}(\Gamma) ;\|\psi\|_{3, \Gamma, \Gamma}=1\right\}}\langle\mu, \psi\rangle_{\Gamma^{\prime}}
$$

where $\langle\cdot, \cdot\rangle_{\Gamma}$ is the duality pairing between $H^{\frac{3}{2}}(\Gamma)$ and $H^{-\frac{1}{2}}(\Gamma)$. The space $H(\operatorname{div} ; \Omega)$ is defined by

$$
H(\operatorname{div} ; \Omega)=\left\{\underline{q}=\left(q_{i}\right)_{1 \leq i \leq n} \in\left(L^{2}(\Omega)\right)^{n} ; \operatorname{div} \underline{q}=\sum_{i=1}^{n} \frac{\partial q_{i}}{\partial x_{i}} \in L^{2}(\Omega)\right\}
$$

and is a Hilbert space with norm

$$
\|\underline{q}\|_{H(\text { div } ; \Omega)}=\|q\|_{0, \Omega}^{2}+\|\operatorname{div} q\|_{0, \Omega^{2}}^{2 / 2} .
$$


$\underline{\text { THEOREM 1.2 }}$. The mapping $\mathrm{q} \longrightarrow q \cdot \underline{\sim}$ defined a priori from $\left(\mathrm{H}^{\mathrm{L}}(\Omega)\right)^{\mathrm{n}}$ into $\mathrm{L}^{2}(\Gamma)$ can be extended to a continuous, linear mapping from $\mathrm{H}\left(\right.$ div; $\Omega$ ) onto $\mathrm{H}^{-\frac{1}{2}}(\Gamma)$. Further we have the following characterization of the norm on $H^{-\frac{1}{2}}(\Gamma)$ :

$$
\|\mu\|_{-\frac{1}{2}, \Gamma}=\inf _{(\underline{q} \in \mathrm{H}(\operatorname{div} ; \Omega) ; q \cdot \nu=\mu)}\|q\|_{H(\operatorname{div} ; \Omega)}
$$

A demonstration of the first part of this theorem can be found in TEMAM (1977, Theorem 1.2, p.9). The characterization of the norm was given by THOMAS (1977, Chapter I). To avoid, whenever possible, working in this space $H^{-\frac{1}{2}}(\Gamma)$ which contains all the functions of $\mathrm{L}^{2}(\Gamma)$, we define, with the aid of Theorem 1.2 , the space

$$
H(\operatorname{div} ; \Omega)=\left(\underline{\mathrm{q}} \in \mathrm{H}(\operatorname{div} ; \Omega) ; \underline{\mathrm{q}} \bullet \underline{\nu} \in \mathrm{L}^{2}(\Gamma)\right)
$$

which is a Hilbert space with norm

$$
\|\underline{q}\|_{H(\operatorname{div} ; \Omega)}=\left\{\left\|q \mathbb{P}_{H(\operatorname{div} ; \Omega)}+\right\| \underline{q} \cdot \underline{\nu} \mathbb{P}_{O, \Gamma}\right\}^{3 / \frac{1}{2}}
$$

We shall make use of the following version of Green's formula :

$$
\begin{array}{ll}
\int_{\Omega}(v \operatorname{div} q+\operatorname{grad} v \cdot q) d x=\int_{\Gamma} v \underline{q} \cdot \nu d \sigma & \text { for } \operatorname{all} v \in H^{1}(\Omega) \text { and } \\
\underline{q} \in H(\operatorname{div} ; \Omega)
\end{array}
$$

Remark 1.1 . The reader not familiar with the notation $H^{\frac{1}{2}}(\Gamma)$ or $H^{-\frac{3}{2}}(\Gamma)$ may find it somewhat surprizing. It is natural in that these spaces can also be defined as Sobolev spaces of order $s$, for $s$ not necessarily an integer. The study of such spaces is not within the scope of this work but may be found in LIONS \& MAGENES (1968, Chapter I) or in ADAMS (1975, Chapter 7).

Other examples of functional spaces will be used in the following, and corresponding trace theorems will be stated. However, before ending this section we would like to give necessary and sufficient conditions for a locally regular scalar function to belong to $H^{1}(\Omega)$ and for a locally regular vector function to belong to $\mathrm{H}(\operatorname{div} ; \Omega)$. More precisely, let $T_{h}$ be a decomposition of $\dot{\Omega}$ into compact sets $T, \bar{\Omega}=\underset{T \in T_{h}}{U} T$, such that the interiors of the sets $T$ of $T_{h}$ are pairwise disjoint and such that the boundary $\partial T$ of an element $T$ of $T_{h}$ is piecewise $C^{1}$. For the sake of simplicity we shall make the following abuses of notation; we shall write 


$$
\begin{array}{ll}
H^{m}(T) \text { for } H^{m}(\stackrel{\circ}{T}), & \|\cdot\|_{m, T} \text { for }\|\cdot\|_{m, \stackrel{\circ}{T}} \\
H(\operatorname{div} ; T) \text { for } H(\operatorname{div} ; \stackrel{\circ}{T}), & \|\cdot\|_{H(\operatorname{div} ; T)} \text { for }\|\cdot\|_{H(\operatorname{div} ; \stackrel{\circ}{T})} ;
\end{array}
$$

etc.

THEOREM 1.3. Let $T_{\mathrm{h}}$ be such a decomposition of $\bar{\Omega}, \bar{\Omega}=\underset{\mathrm{T} \in T_{\mathrm{h}}}{\mathrm{T}}$. A function $v \in L^{2}(\Omega)$, whose restriction $v_{T}$ may be identified with a function $v_{T} \in H^{1}(T)$ for each $T \in T_{h}$, belongs to $\mathrm{H}^{1}(\Omega)$ if and only if for each interface $T^{\prime}=T_{1} \cap T_{2}$ with $\mathrm{T}_{1}, \mathrm{~T}_{2} \in \mathrm{T}_{\mathrm{h}}$, the traces of $\mathrm{v}_{\mathrm{T}_{1}}$ and of $\mathrm{v}_{\mathrm{T}_{2}}$ on $\mathrm{T}^{\prime}$ coincide :

$$
\left.v_{T_{1}}\right|_{T^{\prime}}-v_{T_{2} \mid T^{\prime}} \quad \begin{array}{ll}
\text { for all } T^{\prime}=T_{1} \cap T_{2} \\
\text { with } T_{1}, T_{2} \in T_{h} .
\end{array}
$$

Similarly a function $\mathrm{q} \in\left(\mathrm{L}^{2}(\Omega)\right)^{\mathrm{n}}$, whose restriction $\mathrm{q} / \mathrm{T}$ may be identified with a function $q_{T} \in H$ (div; $T$ ) for each $T \in T_{h}$, belongs to $H$ (div; $\Omega$ ) if and only if for each interface $\mathrm{T}^{\prime}=\mathrm{T}_{1} \cap \mathrm{T}_{2}$ with $\mathrm{T}_{1}, \mathrm{~T}_{2} \in \mathrm{T}_{\mathrm{h}}$, the normal trace of ${ }_{-} \mathrm{T}_{1}$ coincides with the negative of that of $\mathrm{q}_{\mathrm{T}_{2}}$ :

$$
\begin{array}{ll}
\left.\left(\underline{q}_{\mathrm{T}_{1}} \cdot{ }_{-} \mathrm{T}_{1}\right)\right|_{\mathrm{T}^{\prime}}+\left.\left({ }_{-\mathrm{T}_{2}} \cdot{ }_{-\mathrm{T}_{2}}\right)\right|_{\mathrm{T}^{\prime}}=0 \quad \text { for all } \mathrm{T}^{\prime}=\mathrm{T}_{1} \cap \mathrm{T}_{2} \\
\text { with } \mathrm{T}_{1}, \mathrm{~T}_{2} \in T_{\mathrm{h}^{\prime}}
\end{array}
$$

where $\nu_{\mathrm{T}}$ is the unit exterior normal vector of $\mathrm{T}$.

Clearly we have ${ }_{\sim}^{\nu} \mathrm{T}_{1}=-{ }_{-} \mathrm{T}_{2}$ on $\mathrm{T}^{\prime}=\mathrm{T}_{1} \cap \mathrm{T}_{2}$ so that if $\nu$ is any nontrivial vector orthogonal to $T^{\prime}$ we have

$$
\underline{\mathrm{q}}_{\mathrm{T}_{1}} \cdot \underline{\nu}=\underline{\sim}_{\mathrm{T}_{2}} \cdot \underline{\nu} \quad \text { on } \mathrm{T}^{\prime}=\mathrm{T}_{1} \cap \mathrm{T}_{2}
$$




\section{2 - CONFORMING AND EQUILIBRIUM VARIATIONAL FORMULATIONS FOR A MODEL BOUNDARY VALUE} PROBLEM.

Many physical phenomena can be modelled by a system of first order partial. differential equations on a domain $\Omega \subset R^{n}$ of the form

$$
p_{i}=\sum_{j=1}^{n} a_{i j} \frac{\partial u}{\partial x_{j}} \quad \text { in } \Omega, 1 \leq i \leq n,
$$

where the vertnr function $\underline{p}=\left(p_{i}\right)_{1 \leq i \leq n}$ satisfies the equilibrium relation

$$
\operatorname{div} \underset{\sim}{p}+f=0 \quad \text { in } \Omega,
$$

and the boundary conditions are of the form

$$
(\underline{p} \cdot \underline{\nu}-g)+t(u-a)=0 \quad \text { on } \Gamma \text {. }
$$

where $\Gamma$ is the boundary of $\Omega$; cf. DUVAUT \& LIONS (1972, pp. 16-17) for example. For a review of such boundary conditions, cf. DAUTRAY \& LIONS (1985, pp.230-240). The coefficients $a_{i j}$ of the system, defined on $\Omega$ and the function $t$ defined on $\Gamma$ are known functions dependant on the physical problem. The function $f$ defined on $\Omega$ and the functions $g$ and $\bar{u}$ on $\Gamma$ are data functions. The function $t$ is allowed to be infinite, $t=+\infty$, on a part $\Gamma_{0}$ of $\Gamma$ thus implying that $u=\bar{u}$ on $\Gamma_{0}$. The steady state heat equation with convection terms neglected is an example of such a model; $u$ denotes the temperature, $\underline{p}$ is the heat flow, and $\underline{p} \cdot \underline{\nu}, \underline{\sim}$ a unit vector normal to $\Gamma$, is the flux across $\Gamma$. Linear models for elasticity problems involve an analogous system relating the displacement field $\underline{u}=\left(u_{i}\right)_{1 \leq i \leq n}$ and the stress field $\underset{\sigma}{\sigma}=\left(\sigma_{i j}\right)_{1 \leq i, j \leq n}$. Hooke's law gives the stress field in terms of the linearized displacement field; $\left.\epsilon_{i j}(u)=\frac{3 / 2}{\partial x_{j}}+\frac{\partial u_{j}}{\partial x_{i}}\right)$ is the analogue of (2.1).

For these examples corresponding to fundamental problems of engineering, we see that it is at least as important to calculate an approximation of $p$, or even the moments of $\underline{p} \cdot \nu_{\sim}$ on $\Gamma$ or of $\underset{\sim}{p} \cdot \nu$ on a surface contained in $\Omega$ with normal vector $\nu$, as to calculate an approximation of $u$ or of the trace $u_{\Gamma}$ or of the trace of $u$ on a surface lying in $\Omega$. On the other hand, from a strictly theoretical point of view, an apparently obvious approach would seem to be to begin by simplifying the study of system (2.1), (2.2) by eliminating the unknown $\underset{\sim}{p}$, thus considering $u$ as the solution of the second order partial differential equation 


$$
-\sum_{i, j=1}^{n} \frac{\partial}{\partial x_{i}}\left(a_{i j} \frac{\partial u}{\partial x_{j}}\right)=f
$$

in $\Omega$.

The classical methods for the numerical approximation of problem (2.1) - (2.3) are based on the following approach : one determines a function $u_{h}$ satisfying (2.4) in a more or less weak sense--using for instance standard finite difference or finite element methods. Once the function $u_{h}$ has been calculated, if it is necessary to obtain an approximation of $p$, the simplest procedure is to consider the function $p_{h}$ whose $i^{\text {th }}$ component, $1<i \leq n$, is given by $\left(p_{h}\right)_{i}-\sum_{j=1}^{n} a_{i j} \frac{\partial u_{h}}{\partial x_{j}}$. This naive treatment has the inconvenience of producing a function ${\underset{w}{h}}_{\text {which satisfies }}$ only in a very weak sense the equilibrium condition and for which the two normal traces ${\underset{\sim}{h}}_{h} \nu_{-T}$, across an interface $T \cdot \subset \Omega$ do not agree. Experience shows that it is better, a priori, to choose a method which solves directly for the desired quantities rather than to try to obtain them in an indirect fashion as in the example above. Our objective here is to propose several such methods.

We shall consider in this chapter, by way of introduction, the case in which the coefficient functions $a_{i j}$ are constant in $\Omega$, and the matrix $\left(a_{i j}\right)$ is symmetric and positive definite. Thus the relations (2.1) are invertible:

$$
\frac{\partial u}{\partial x_{i}}=\sum_{j=1}^{n} A_{i j} P_{j}, \quad 1 \leq i \leq n
$$

and the inverse matrix $\left(A_{i j}\right)$ is also symmetric and positive definite. For the moment we shall restrict our attention to the two following model problems :

The homogeneous Dirichlet problem. Given $f$ in. $L^{2}(\Omega)$, find $\underline{p} \in H($ div; $\Omega$ ) and $u \in H_{0}^{1}(\Omega)$ satisfying (2.1) and (2.2).

The homogeneous Neumann problem. Given $f$ in $L^{2}(\Omega)$ with $\int_{\Omega} f d x=0$, find $\underline{p} \in H_{0}(\operatorname{div} ; \Omega)$ and $u \in H^{1}(\Omega) / R$ satisfying (2.1) and (2.2), where for the sake of simplicity we suppose that $\Omega$ is connected.

Just as $H_{0}^{1}(\Omega)$ denotes the kernel of the trace mapping of $H^{1}(\Omega)$ into $L^{2}(\Gamma)$, $\mathrm{H}_{0}(\mathrm{div} ; \Omega)$ denotes the kernel of the normal trace mapping of $\mathrm{H}(\mathrm{div} ; \Omega)$ into $\mathrm{L}^{2}(\Gamma)$. $A$ specific representative of the equivalence class of $u$ in the quotient space $\mathrm{H}^{1}(\mathrm{a}) / \mathrm{R}$, for $\mathrm{u}$ a solution of the homogeneous Neumann problem, may be chosen by requiring further that a relation such as $\int_{\Omega} u d x=0$ or such as $\int_{\Gamma} u d x=0$ be satisfied. (Using the standard abuse of notation we write $u$ for an element of $H^{1}(\Omega)$ as well as for its equivalence class in $\left.H^{1}(\Omega) / R\right)$. 
The solution $(p, u)$ in the case of the homogeneous Dirichlet or the homogeneous Neumann problem may be characterized as the unique solution of a minimization problem. For the homogeneous Dirichlet problem put

$$
W^{f}=\{q \in H(\operatorname{div} ; \Omega) ; \operatorname{div} q+f=0\}, \quad V=H_{0}^{1}(\Omega),
$$

and for the homogeneous Neumann problem

$$
W^{f}=\left\{\underline{q} \in H_{0}(\operatorname{div} ; \Omega) ; \operatorname{div} q+f=0\right\}, \quad V=H^{1}(\Omega) / R
$$

Then in either case $(\underline{p}, u)$ is the unique element of $w^{f} \times V$ satisfying (2.1) or equivalently (2.5). On $\mathrm{w}^{\mathrm{f}} \times \mathrm{V}$ define the functional

$$
I(q, v)=\frac{1}{2} \int_{\Omega} \sum_{i=1}^{n}\left(\left(q_{i}-\sum_{j=1}^{n} a_{i j} \frac{\partial v}{\partial x_{j}}\right)\left(\sum_{k=1}^{n} A_{i k} q_{k}-\frac{\partial v}{\partial x_{i}}\right)\right) d x .
$$

For each pair $(q, v)$ in $w^{f} \times v$ we have

$$
I(q, v)=\frac{1}{2} \int_{\Omega} \sum_{i, k=1}^{n}\left(\left(q_{i}-\sum_{j=1}^{n} a_{i j} \frac{\partial v}{\partial x_{j}}\right)\left(A_{i k}\left(q_{k}-\sum_{\ell=1}^{n} a_{k l} \frac{\partial v}{\partial x_{l}}\right)\right)\right) d x \geq 0,
$$

with the equality being realized, as $[A]$ is positive definite, only in case (q,v) satisfies the relation $q_{i}=\sum_{j=1}^{n} a_{i j} \frac{\partial v}{\partial x_{j}}$ for all $i, i=1, \ldots, n$; i.e. if $(\underline{q}, v)$ satisfies (2.1). Thus the solution $(\underline{p}, u)$ of (2.1) - (2.2) with the homogeneous Dirichlet or the homogeneous Neumann condition is the unique element of $W^{f} \times V$ satisfying

$$
I(\underline{p}, u)=0
$$

or in other words the unique element minimizing $I$ on $\mathrm{W}^{f} \mathrm{xV}$. This presentation was inspired by that in BARLOW (1986).

With this characterization of the solution $(\underset{p}{p}, u)$ of $(2.1)-(2.3)$, it is natural to try to construct an approximation in the following manner. Let $v_{h}$ be a finite dimensional subspace of $V$ and $w_{h}^{f}$ a finite dimensional, affine subspace of $H(\operatorname{div} ; \Omega)$ or $H_{0}(\operatorname{div} ; \Omega)$ contained in $W^{f}$. We consider the following problem : find $\left(\underline{p}, u_{h}\right)$ satisfying 
(2.9) $\quad\left\{\begin{array}{l}\left(\underline{\sim}_{h}, u_{h}\right) \in w_{h}^{f} \times v_{h}, \\ I\left(\underline{p}_{h}, u_{h}\right)=\underset{\left(q_{h}, v_{h}\right) \in w_{h}^{f} \times v_{h}}{\inf } I\left(\underline{q}_{h}, v_{h}\right) .\end{array}\right.$

Problem (2.9) has a unique solution $\left(\underline{p}_{h}, u_{h}\right) ;$ the uniqueness is an immediate consequence of the characterizations given by (2.15) and (2.16) below. Furthermore, the calculation of the value $I\left(\underline{p}_{h}, u_{h}\right)>0$ furnishes, a posteriori, a control of the error committed in the approximation.

Formulation (2.9) thus seems to provide a natural coupling between the approximations $\underline{\sim}_{h}$ of $\underset{\sim}{p}$ and $u_{h}$ of $u$. In fact it does not at all. To see this one has only to note that, with the choice (2.6D) as well as with the choice $(2.6 \mathrm{~N})$; we have

$$
I(q, v)=J_{*}(q)+J^{f}(v)
$$

with

$$
\begin{aligned}
& J^{f}(v)=\frac{1}{2} \int_{\Omega} \sum_{i, j=1}^{n} a_{i j} \frac{\partial v}{\partial x_{j}} \frac{\partial v}{\partial x_{i}} d x-\int_{\Omega} f v d x, \\
& J_{*}(q)=\frac{1}{2} \int_{\Omega} \sum_{i, j=1}^{n} A_{i j} q_{j} q_{i} d x .
\end{aligned}
$$

Hence if $(\underline{p}, u)$ is the solution of $(2.8)$, then $u$ is characterized as being the unique solution of the minimization problem : find u satisfying

$$
\left\{\begin{array}{l}
u \in v ; \\
J^{f}(u)=\inf _{v \in V} J^{f}(v) ;
\end{array}\right.
$$

and $\underline{p}$ as being that of the minimization problem : find $\underline{p}$ satisfying

$$
\left\{\begin{array}{l}
\underline{p} \in W^{f} ; \\
J_{*}(\underline{p})=\inf _{q \in W^{f}} J_{*}(q) .
\end{array}\right.
$$

Since $I(\underline{p}, u)=0$, we have the relation

$$
J^{f}(u)+J_{*}(\underline{p})=0
$$


known in mechanics as the complementary energy principle.

The solution $\left(\underline{p}_{h}, u_{h}\right)$ of $(2.9)$ is characterized with the aid of the following two minimization problems : find $u_{h}$ satisfying

$$
\left\{\begin{array}{l}
u_{h} \in v_{h}, \\
J^{f}\left(u_{h}\right)=\inf _{\substack{v_{n} \in v_{h}\\
}} J^{f}\left(v_{h}\right)
\end{array}\right.
$$

find $\stackrel{\sim}{p}_{h}$ satisfying

$$
\left\{\begin{array}{l}
{\underset{\sim}{p}}_{h} \in w_{h}^{f}, \\
J_{*}\left(\underline{p}_{h}\right)=\inf _{q_{h} \in W_{h}^{f}} J_{*}\left(q_{h}\right) .
\end{array}\right.
$$

Since the approximate solution $u_{h} \in v_{h}$ is an element of $v$, we say that $u_{h}$ is obtained by a conforming approximation method. Similarly, since the approximate solution ${ }_{\sim}^{p} \in W_{h}^{f}$ is an element of $w^{f}$ we say that $\underline{p}_{h}$ is obtained by an equilibrium approximation method. If $\bar{J}$ is the energy defined by $\bar{J}=J^{f}(u)=-J_{*}(\underset{\sim}{)}$ ), cf. (2.15), we remark that the conforming approximation permits us to calculate an approximation $J^{f}\left(u_{h}\right)$ of $j$ which is of necessity excessive, whereas the equilibrium approximation permits us to calculate an approximation $-J_{*}\left(p_{h}\right)$ of $\bar{J}$ which necessarily too small :

$$
-J_{*}\left(\underline{p}_{h}\right) \leq-J_{*}(\underline{p})=\bar{J}=J^{f}(u) \leq J^{f}\left(u_{h}\right)
$$

Remark 2.1. The possibility to thus obtain an a posteriori bound on the energy $\vec{J}$ is what motivated the development of equilibrium approximation methods, $c f$. FRAEIJS DE VEUBEKE (1965), (1973), (1975), FRAEIJS DE VEUBEKE \& HOGGE (1972). 
Remark 2.2 . Let $g$ be a function in $L^{2}(\Gamma)$ satisfying $\int_{\Gamma} g$ ds $=0$. If $(\underline{p}, u)$ is a solution of the system

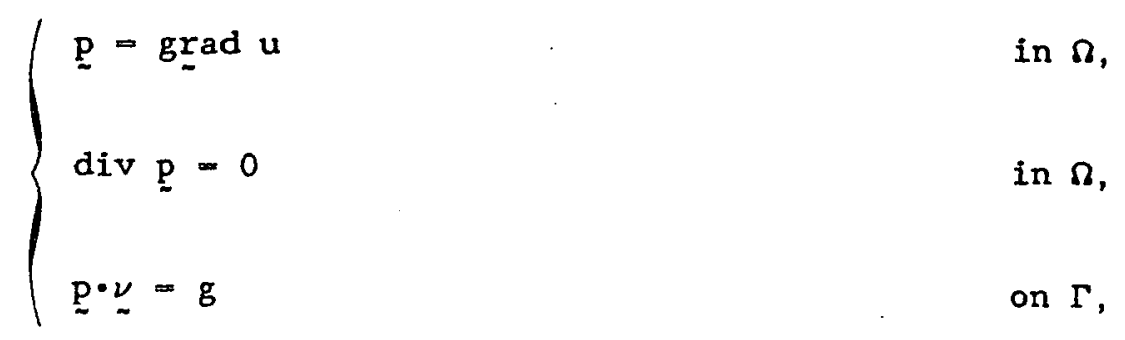

for $\Omega$ a connected open subset of $R^{n}$, then as above one can verify that the function $u$, defined to within a constant, minimizes on $H^{1}(\Omega) / \mathbf{R}$ the functional

$$
J_{g}(v)=\frac{1}{2} \int_{\Omega}|\operatorname{grad} v|^{2} d x-\int_{\Gamma} g v d \sigma,
$$

whereas the vector function p minimizes the functional

$$
J_{*}(q)=\frac{1}{2} \int_{\Omega}|q|^{2} d x
$$

on the affine manifold of vector functions $q$ with div $q=0$ on $\Omega$ and $q \cdot \underline{\nu}=g$ on $\Gamma$. If $u$ minimizes $J_{g}(v)$ then we have div ( $\underset{\sim}{\operatorname{rad}} u$ ) $=0$; and if $\underline{p} \operatorname{minimizes} J_{*}(q)$, we have rot $\underline{p}=0$ since then $\underline{p}$ has a scalar potential. These principles are known in fluid mechanics as Dirichlet's principle and Kelvin's principle, respectively ; cf SERRIN J. (1959, Section 24). In the standard terminology of fluid mechanics, the

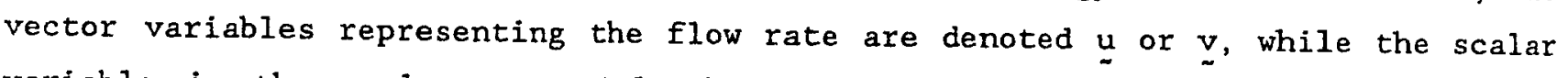
variable is the scalar potential of the velocity field or the pressure and is generally denoted $\phi, \psi$, or $p$.

Remark 2.3. We shall see later on, cf. Remark 3.1; that the two minimization problems (2.13) and (2.14) (or similarly (2.19) and (2.20)) are dual problems in the sense of the mathematical theory of duality in convex anaiysis. Usually (2.13) is considered to be the primal problem and (2.14) its dual. In keeping with standard practice we shall say that a formulation is primal, respectively dual, when the elimination of certain variables yields the conforming formulation (2.13), respectively the equilibrium formulation (2.14). 
To conclude this presentation of conforming and equilibrium approximations we give the variational formulations associated with these minimization problems :

Conforming variational formulation. Find a solution $u$ of

(2.21) $\left\{\begin{array}{l}u \in v, \\ \sum_{i, j=1}^{n} \int_{\Omega} a_{i j} \frac{\partial u}{\partial x_{j}} \frac{\partial v}{\partial x_{i}} d x=\int_{\Omega} f v d x \quad \text { for all } v \in v,\end{array}\right.$

which leads one to seek an approximation $u_{h}$ of u satisfying

$$
\left\{\begin{array}{l}
u_{h} \in v_{h}, \\
\sum_{i, j=1}^{n} \int_{\Omega} a_{i j} \frac{\partial u_{h}}{\partial x_{j}} \frac{\partial v_{h}}{\partial x_{i}} d x=\int_{\Omega} f v_{h} d x \quad \text { for all } v_{h} \in v_{h} .
\end{array}\right.
$$

Equilibrium variational formulation. Find a solution $\underline{p}$ of

$$
\left\{\begin{array}{l}
\underline{p} \in \mathrm{w}^{\mathrm{f}}, \\
\sum_{i, j=1}^{\mathrm{n}} \int_{\Omega} A_{i j} \mathrm{p}_{j} q_{i} \mathrm{dx}=0
\end{array}\right.
$$

which leads one to seek an approximation ${\underset{\sim}{h}}_{h}$ of $\underset{\sim}{p}$ satisfying

$$
\begin{cases}p_{h} \in w_{h}^{f}, & \text { for all } q_{h} \in w_{h}^{0} ; \\ \sum_{i, j=1}^{n} \int_{\Omega} A_{i j} p_{h j} q_{h i} d x=0 & \text {. }\end{cases}
$$

where $w^{O}$, respectively $w_{h}^{O}$, is $w^{f}$, respectively $w_{h}^{f}$, for $f=0$ and hence is a subspace of $\mathrm{H}(\operatorname{div} ; \Omega)$ or $\mathrm{H}_{0}(\operatorname{div} ; \Omega)$ depending on whether the problem considered is the homogeneous Dirichlet problem or the homogeneous Neumann problem. 


\section{3 - FIRST EXAMPLES OF MIXED AND HYBRID FORMULATIONS}

The actual construction of a solution $\underline{\mathrm{p}}_{h}$ of problem (2.24) (equilibrium formulation) poses several practical difficulties. If $T_{h}$ is a triangulation of $\bar{n}$, $\bar{\Omega}=\underset{T \in T_{h}}{U} T$, and $W_{h}^{0}$ is a finite dimensional subspace of $w^{0}$ associated with $T_{h}$, then for a test function $q_{h}$ to belong to $w_{h}^{0}$ it must satisfy the following two constraints :

i) The normal traces of $q_{h}$ must be "continuous across the interface" between any two abutting finite elements $T_{1}$ and $T_{2}$ of $T_{h}$, i.e. $\underline{-}_{h} \cdot{ }_{-} T_{1}$ and $-q_{h} \cdot \nu_{*} T_{2}$ must coincide on $T^{\prime}=T_{1} \cap T_{2}$ (cf. the second part of Theorem 1.3).

ii) Within each finite element $T$ of $T_{h}$ the divergence of $q_{h}$ must vanish; i.e. $\operatorname{div}\left(\underline{q}_{h \mid T}\right)=0$ for all $T \in T_{h}$; thus, in particular $\int_{\partial T} \underline{q}_{h} \nu_{-T} d \sigma=0$.

We do not intend to attack head on the practical problem of constructing a basis for the subspace $w_{h}^{0}$. To circumvent this difficulty we shall free ourselves of one of the constraints that should be satified by the test functions by using the technique of Langrangian multipliers.

The dual mixed formulation. The affine subspace defined by (2.6D) in the case of the homogeneous Dirichlet problem and by $(2.6 \mathrm{~N})$ in the case of the homogeneous Neumann problem may be characterized as being the set of functions $q \in W$ satisfying the constraint $\operatorname{div} q+f=0$ in $\Omega$, where $W$ is the space defined by

$$
\begin{aligned}
& W=H(\operatorname{div} ; \Omega), \\
& W=H_{0}(\operatorname{div} ; \Omega) .
\end{aligned}
$$

Thus we are led to substitute for the minimization problem (2.14) the saddle point problem of the Lagrangian $\mathcal{L}_{*}(\cdot, \cdot)$ defined on $W \times L^{2}(\Omega)$ by

$$
L_{*}(q, v)=J_{*}(q)+\int_{\Omega}(\operatorname{div} q+f) v d x
$$

Thus we seek $(\underline{p}, u) \in W \times L^{2}(\Omega)$ satisfying 


$$
L_{*}(\underline{p}, \mathrm{v}) \leq L_{*}(\underline{\mathrm{p}}, \mathrm{u}) \leq \mathscr{L}_{*}(\mathrm{q}, \mathrm{u})
$$$$
\text { for all }(q, v) \in W \times L^{2}(\Omega) \text {. }
$$

The solution is characterized as being the solution of the system

$$
\begin{cases}(\underline{p}, u) \in W \times L^{2}(\Omega), & \text { for all } q \in W \\ \sum_{i, j=1}^{n} \int_{\Omega} A_{i j} p_{j} q_{i} d x+\int_{\Omega} u \text { div } q d x=0 & \\ \int_{\Omega}(d i v \underset{\sim}{p}) v d x=-\int_{\Omega} f v d x & \text { for all } v \in L^{2}(\Omega) .\end{cases}
$$

If $(\underline{p}, u)$ satisties $(3.4)$ it is clear that $\underline{p}$ satisfies $(2.23)$.

Denote, for the moment, by $\hat{u}$ the solution of (2.21). Using Green's formula together with relation (2.5), we see that

$$
\begin{aligned}
\sum_{i, j=1}^{n} \int_{\Omega} A_{i j} p_{j} q_{i} d x & =\int_{\Omega}(\operatorname{grad} \hat{u}) \cdot \underline{q} d x \\
& =-\int_{\Omega} \hat{u} \text { div } \underline{q} d x \quad \text { for all } \underline{q} \in W
\end{aligned}
$$

Hence the Lagrange multiplier associated with the constraint div $q=f$ is none other than

$$
u=\hat{u} .
$$

The primal mixed formulation. In virtue of (3.5), the solution $(p, u)$ actually belongs to $W \times V$. Using Green's formula we establish that the solution $(p, u)$ satisfies

$$
\begin{cases}\sum_{i, j=1}^{n} \int_{\Omega} A_{i j} p_{j} q_{i} d x-\int_{\Omega}(\operatorname{grad} u) \cdot q d d x=0 & \text { for all } q \in W \\ -\int_{\Omega} \underset{\sim}{p} \cdot \underset{\sim}{\operatorname{grad} v d x}=-\int_{\Omega} f v d x & \text { for all } v \in v\end{cases}
$$

As $W$ is dense in $\left(L^{2}(\Omega)\right)^{n}$, the pair $(\underline{p}, u)$ is the solution of the system 
$(3.6)$

$$
\begin{cases}(\underline{p}, u) \in\left(L^{2}(\Omega)\right)^{n} \times v, & \text { for all } q \in\left(L^{2}(\Omega)\right)^{n}, \\ \sum_{i, j=1}^{n} \int_{\Omega} A_{i j} p_{j} q_{i} d x-\int_{\Omega}(\underset{\sim}{\operatorname{rad} u) \cdot q d x=0} & \text { for all } v \in V .\end{cases}
$$

This formulation, (3.6), can be interpreted as being a characterization of the saddle point of the functional $L^{f}(\cdot, \cdot)$ defined on $\left(L^{2}(\Omega)\right)^{n} \times V$ by

$$
\mathcal{L}^{\mathrm{f}}(\underline{\mathrm{q}}, \mathrm{v})-j_{*}(\underline{q})-\int_{\Omega} \underline{q} \cdot \operatorname{grad} \mathrm{v} \mathrm{dx}+\int_{\Omega} f \mathrm{v} \mathrm{dx},
$$

where $\tilde{J}_{*}$ is the continuous extension of $J_{*}$ to the space $\left(L^{2}(\Omega)\right)^{n}$. This functional can be found in the literature written in the form

$$
\begin{aligned}
(3.7 \text { bis }) \quad \mathcal{L}^{f}(\underline{q}, v)=-\frac{1}{2} \sum_{i, j=1}^{n} \int_{\Omega} A_{i j} q_{j} q_{i} d x+\sum_{i=1}^{n} \int_{\Omega}\left(\sum_{j=1}^{n} A_{i j} q_{j}-\frac{\partial v}{\partial x_{i}}\right) q_{i} d x+ \\
+\int_{\Omega} f v d x
\end{aligned}
$$

The elimination of $\underline{p}$ from formulation (3.6) gives us again the conforming variational formulation (2.21) of the problem. This type of method is analyzed in BABUSKA, ODEN, \& LEE (1977). Such methods have proved useful in theoretical studies such as the development of new models for nonlinear elasticity, cf. CIARLET \& DESTUYNDER (1979a), (1979b), DESTUYNDER (1986).

Remark 3.1. We have on the one hand

$$
\sup _{v \in V} L^{f}(q, v)= \begin{cases}J_{\star}(q) & \text { if } q \in W^{f} \\ +\infty & \text { otherwise }\end{cases}
$$

and on the other

$$
\inf _{q \in W} f^{f}(q, v)=-\frac{1}{2} \sum_{i, j=1}^{n} \int_{\Omega} a_{i j} \frac{\partial v}{\partial x_{j}} \frac{\partial v}{\partial x_{i}} d x+\int_{\Omega} f v d x .
$$

We deduce that

$$
\inf _{q \in W} \sup _{v \in V} L^{f}(q, v)=\inf _{\underline{q} \in W^{f}} J_{*}(q)=J_{*}(\underline{p})
$$


and that

$$
\sup _{\mathrm{v} \in \mathrm{V}} \inf _{\mathrm{q} \in \mathrm{W}} \mathcal{L}^{f}(\underline{q}, \mathrm{v})-\inf _{\mathrm{v} \in \mathrm{V}} J^{f}(\mathrm{v})=-J^{f}(u)
$$

Then using the complementary energy principle (2.15), we obtain

$$
\inf _{q \in W^{f}} J_{*}(q)=\inf _{q \in W} \sup _{v \in V} L^{f}(q, v)=\sup _{v \in V} \inf _{q \in W} L^{f}(q, v)=-\inf _{v \in V} J^{f}(v) .
$$

In this form we recognize the minimization problems (2.13) and (2.14) as dual problems in the sense of optimization theory, cf. for example CIARLET (1982, p.222).

The dual hybrid formulation. To a decomposition $T_{h}$ of $\bar{\Omega}, \bar{\Omega}=U \mathrm{~T}$, is associated the affine subspace $\mathrm{Y}^{f}$ of $\left(\mathrm{L}^{2}(\Omega)\right)^{\mathrm{n}}$ defined by $\mathrm{T} \in T_{\mathrm{h}}$

$$
\begin{aligned}
\mathrm{Y}^{\mathrm{f}}= & \left(\mathrm{q} \in\left(\mathrm{L}^{2}(\Omega)\right)^{\mathrm{n}} ; \forall \mathrm{T} \in T_{\mathrm{h}}, \operatorname{div}\left(q_{\mid \mathrm{T}}\right)+\mathrm{f}_{\mid \mathrm{T}}=0\right. \\
& \text { in the distributional sense }) .
\end{aligned}
$$

In other words, $\mathrm{Y}^{\mathrm{f}}$ is the set of vector functions $q \in\left(L^{2}(\Omega)\right)^{n}$ satisfying for each $\mathrm{T} \in T_{\mathrm{h}}$

$$
\int_{T} q \cdot \operatorname{grad} v_{T} d x=\int_{T} f v_{T} d x \quad \text { for all } v_{T} \in H_{0}^{1}(T)
$$

For each $T \in T_{h}$, the restriction $q_{T}$ of the function $q \in Y^{f}$ to $T$ belongs to the space $H$ (div; $\mathrm{T})$. Further, the normal trace $\mathrm{q}_{\left.\right|_{\mathrm{T}}{ }_{*}{ }_{\mathrm{T}}}$ is defined, cf. Theorem 1.2 , to be an element of $H^{-\frac{1}{2}}(\partial T)$, the dual space of $H^{\frac{1}{2}}(\partial T)$; we denote by $\langle\cdot, \cdot\rangle_{\partial T}$ this duality. We ascertain that the affine subspace $\mathrm{W}^{f}$ may be characterized as being the set of functions $q \in \mathrm{Y}^{\mathrm{f}}$ satisfying the constraint

$$
\sum_{T \in T_{h}}\left\langle q_{T} \cdot \nu_{T}, v\right\rangle_{\partial T}=0 \quad \text { for all } v \in V
$$

This leads us to associate to the decomposition $T_{h}$ of $\bar{\Omega}$ the Lagrangian $D_{*}(\cdot, \cdot)$ defined on $\mathrm{Y}^{\mathrm{f}} \times \mathrm{V}$ by

$$
D_{*}(\underline{q}, v)=J_{*}(\underline{q})-\sum_{T \in T_{h}}\left\langle\left. q_{T} \cdot\right|^{*}, v\right\rangle_{\partial T} .
$$


(The choice of $D_{*}(q, v)$ as given in (3.10) instead of as $J_{*}(q)+\sum_{T \in T}\left\langle q_{q_{T}} \cdot \nu_{-T}, v\right\rangle_{\partial T}$ is made simply to obtain (3.13) below). Thus we seek an element $(\underset{\sim}{p}, u) \in Y^{f} \times V$ satisfying

$$
D_{*}(\underline{p}, \mathrm{v}) \leq D_{*}(\underline{\mathrm{p}}, \mathrm{u}) \leq D_{*}(\underline{\mathrm{q}}, \mathrm{u}) \quad \text { for } \operatorname{all}(\underline{\mathrm{q}}, \mathrm{v}) \in \mathrm{Y}^{\mathrm{f}} \times \mathrm{v}
$$

A pair $(\underline{p}, u)$ is such an element if and only if it is a solution of the system

$$
\begin{cases}(\underline{p}, u) \in Y^{f} \times v, & \text { for all } q \in Y^{0} \\ \sum_{i, j=1}^{n} \int_{\Omega} A_{i j} p_{j} q_{i} d x+\sum_{T \in T_{h}}\left\langle q_{T} \cdot \nu_{T}, u>_{\partial T}=0\right. & \text { for all } v \in v ; \\ \sum_{T \in T_{h}}\left\langle\underline{p}_{T} \cdot \nu_{T}, v\right\rangle_{\partial T}=0 & \text { for }\end{cases}
$$

where $Y^{0}$ is $Y^{f}$ for $f \equiv 0$. If $(\underset{p}{p}, u)$ is a solution of problem (3.12), then $p$ is the solution of (2.22). It is clear that $u$ is not uniquely defined as an element of $\mathrm{V}$; only its traces on the interfaces are determined by the first equation of (3.12). However, if $\hat{u}$ is the solution of (2.20), we can choose

$$
u=\hat{a}
$$

Remark 3.2 . In fact with the dual hybrid formulation the Lagrangian multiplier should. not be considered as a function in $V$ defined on all $\Omega$ but as an element of the product space $\prod_{T \in T_{h}} H^{\frac{3}{2}}(\partial T)$ satisfying certain matching conditions at the interfaces. This will be the point of view taken for the analysis of the method given in Chapter 5. A variation of considerable practical interest consists of taking the multiplier to be an element of $\Pi L^{2}(\partial T)$ satisfying again certain matching conditions at the interfaces. $\quad T \in T_{h}$

The primal hybrid formulation. To a decomposition $T_{h}$ of $\bar{\Omega}$ we associate in a manner similar to the above a subspace $\mathrm{X}$ of $\mathrm{L}^{2}(\Omega)$ defined by 
(3.14)

$$
X=\left\{v \in L^{2}(\Omega), \forall T \in T_{h},\left.\quad\right|_{T} \in H^{2}(T)\right\}
$$

The space $V$ may be characterized as being the space of functions $v \in X$ satisfying the constraint

$$
\sum_{\mathrm{T} \in T_{\mathrm{h}}}\left\langle\mathrm{q} \cdot \nu^{-} \mathrm{I}_{\mathrm{T}}, \mathrm{v}_{\mathrm{T}}\right\rangle_{\partial \mathrm{T}}=0 \quad \text { for all } \mathrm{q} \in \mathrm{W} .
$$

Thus we replace the minimization problem (2.13) by the saddle point problem for the Lagrangian $D^{f}(\cdot, \cdot)$ defined on $\mathrm{X} \times \mathrm{W}$ by

$$
D^{f}(v, q)=j^{f}(v)-\sum_{T \in T_{h}}\left\langle\underline{q} \cdot \underline{\nu}_{T}, v_{T}\right\rangle_{\partial T},
$$

where $J^{f}$ is the extension of $J^{f}$ to $X$ defined as follows :

$$
\bar{J}^{f}(v)=\frac{1}{2} \sum_{T \in T_{h}}\left(\int_{T} \sum_{i, j=1}^{n} a_{i j} \frac{\partial v}{\partial x_{j}} \frac{\partial v}{\partial x_{i}} d x\right)-\int_{\Omega} f v d x .
$$

Thus we seek a solution $(\underline{p}, u)$ of the system

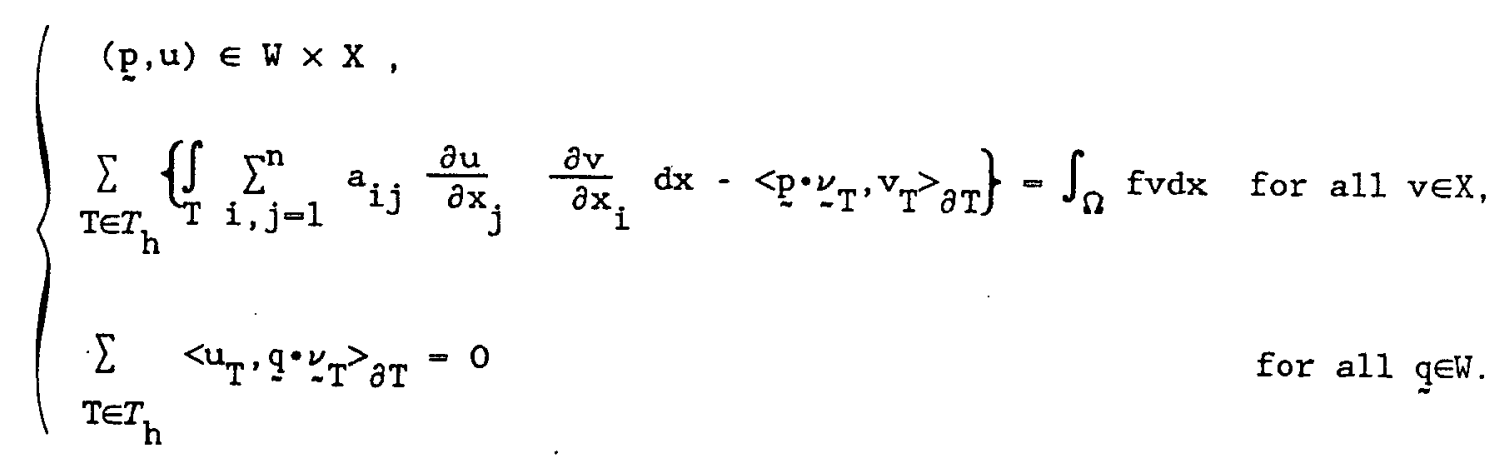

The solution $(\underline{p}, u)$ of problem (3.17) is such that $u$ is a solution of problem (2.21) and if $\hat{p}$ is the solution of (2.23) we may choose

$$
\underline{\mathrm{p}}=\hat{\mathrm{p}}_{-} \text {, }
$$

as indeed only the normal traces of $p$ on the interfaces are determined by (3.17).

Remark 3.3 . The hybrid formulations have been presented here as subordinate to a decomposition $T_{h}$ of $\bar{\Omega}$ which will, of course, coincide with the triangulation of $\bar{\Omega}$ by elements $T$ on which will be constructed approximations of functions. One 
can, however, generalize this type of hybrid formulation to one subordinate to an arbitrary decomposition of $\bar{\Omega}$ into subdomains. A particular case is that for which the decomposition is the simplest possible, a decomposition into a single "subdomain". In this case the primal hybrid formulation of the homogeneous Dirichlet problem becomes : find a saddle point $(u, \lambda) \in H^{1}(\Omega) \times H^{-\frac{1}{2}}(\Gamma)$ of the Lagrangian $f^{f}(\cdot, \cdot)$ defined by $f^{f}(v, \mu)=J^{-f}(v)-\langle\mu, v\rangle_{\Gamma}$ for all $(v, \mu) \in H^{1}(\Omega) \times$ $H^{-\frac{1}{2}}(\Gamma)$. It was for this type of formulation, adapted to a nonhomogeneous Dirichlet problem, that the first mathematical analysis of a finite element method with Lagrangian multipliers was given, cf. BABUSKA (1973).

\section{4 - GENERAL ORIENTATION}

The elementary examples of mixed formulations and of hybrid formulations presented in Section 3 may all be written in the following general form : find a pair $(\phi, \lambda)$ satisfying

(4.1) $\begin{cases}\phi \in W, \lambda \in M, & \\ a(\phi, \psi)+b(\psi, \lambda)=f(\psi) & \text { for all } \psi \in W, \\ b(\phi, \mu) & \text { for all } \mu \in M,\end{cases}$

where $W$ and $M$ are Hilbert spaces, $a(\cdot, \cdot)$ is a symmetric bilinear form on $W \times W$, $b(\cdot, \cdot)$ is a bilinear form on $W \times M, f$ is a linear form on $W$, and $g$ is a linear form on $M$. Then given a finite dimensional subspace $W_{h}$ of $W$ and a finite dimensional subspace $M_{h}$ of $M$ we consider the problem : find a pair $\left(\phi_{h}, \lambda_{h}\right)$
satisfying

$$
\left\{\begin{array}{l}
\phi_{h} \in W_{h}, \lambda_{h} \in \mathbb{M}_{h} \\
a\left(\phi_{h}, \psi_{h}\right)+b\left(\psi_{h}, \lambda_{h}\right)=f\left(\psi_{h}\right) \\
b\left(\phi_{h}, \mu_{h}\right) \\
=g\left(\mu_{h}\right)
\end{array}\right.
$$

$$
\begin{aligned}
& \text { for a11 } \psi_{h} \in W_{h}, \\
& \text { for al1 } \mu_{h} \in M_{h} .
\end{aligned}
$$

This finite dimensional problem may then be posed in the obvious manner as a problem of solving a linear system of $N_{h}$ equations in $N_{h}$ unknowns, where

$$
N_{h}=\operatorname{dim} W_{h}+\operatorname{dim} M_{h} .
$$

The matrix of this system is symmetric but can not be positive definite. Thus the first problem is to find sufficient conditions, bearing on the pair $\left(W_{h}, M_{h}\right)$ of subspaces, for the matrix of the associated linear system to be invertible. In a 
more general way Chapter III treats the numerical analysis of this type of problem in an abstract setting. The a priori estimates of the exror committed in approximating the solution $(\phi, \lambda)$ of $(4.1)$ by the solution $\left(\phi_{h}, \lambda_{h}\right)$ of $(4.2)$ are obtained as a function of the error of approximation of $(\phi, \lambda)$ in $w_{h} \times M_{h}$.

In the examples given in Section 3 , the spaces $W$ and $M$ appearing in the mixed or hybrid formulations are either $L^{2}(\Omega), H^{1}(\Omega)$, or closed subspaces of these spaces for the space of scalar functions and either $\left(L^{2}(\Omega)\right)^{n}, H(d i v ; \Omega)$, or closed subspaces of these spaces for the space of vector functions. Chapter II contains the major results concerning interpolation by finite elements of scalar functions in $H^{1}(\Omega)$ and of vector functions in $H(d i v ; \Omega)$.

Chapters II and III are independent. Both are fundamental for the analysis of the examples developed in the subsequent chapters.

Chapters IV and V treat essentially the approximation of the model problem governed by the first order system of partial differential equations

$$
\begin{aligned}
& \text { (4.4) } p_{i}=\sum_{j=1}^{n} a_{i j} \frac{\partial u}{\partial x_{j}}, \quad 1 \leq i \leq n, \\
& \text { (4.5) } \quad \operatorname{div} \underset{\sim}{p}+f=0,
\end{aligned}
$$

posed on a bounded, open domain $\Omega \subset R^{n}$ with $n=2$ or $n=3$ with boundary $\Gamma$ piecewise $C^{1}$. The coefficients $a_{i j}$ are supposed to be bounded and measurable in $\Omega$ and to satisfy the uniform ellipticity hypothesis

$$
\sum_{i, j=1}^{n} a_{i j}(x) \xi_{i} \xi_{j} \geq \alpha_{p}|\xi|^{2} \text { a.e. on } \Omega \quad \text { for all } \xi \in \mathbb{R}^{n}
$$

for some $\alpha_{p}>0$. Unlike in Sections 2 and 3 , we shall not suppose the symmetry of [a], i.e. that $a_{i j}=a_{j i}$. Also we shall not maintain throughout the following the notation particular to Sections 2 and 3 .

With the above hypotheses concerning the coefficients $a_{i j}$ the matrix [a] may be inverted to obtain

$$
\frac{\partial u}{\partial x_{i}}=\sum_{j=1}^{n} A_{i j} p_{j}, \quad 1 \leq i \leq n
$$

the coefficients $A_{i j}$ are then bounded and measurable in $\Omega$ and satisfy the uniform ellipticity hypothesis 


\subsection{2}

(4.8) $\quad \sum_{i, j=1}^{n} A_{i j}(x) \xi_{i} \xi_{j} \geq \alpha_{d}|\xi|^{2}$ a.e. on $\Omega \quad$ for all $\xi \in \mathbb{R}^{n}$,

for some $\alpha_{d}>0$.

Chapter IV is concerned with mixed methods. Primal mixed methods are treated rather rapidly, the dual mixed methods in some more detail. Several algorithms for the solution of the linear system are also given here. In Chapter $V$ hybrid methods are studied. Primal hybrid and dual hybrid methods as well as the method of hybridization of mixed methods are included. In Chapter 6 some further examples of mixed methods and of hybrid methods are mentioned, and some extensions and variants of the theory in the literature are cited. 


\section{CHA P TER II}

\section{FINITE ELEMENT TYPE INTERPOLATION OF SCALAR AND VECTORIAL FUNCTIONS}

\section{5 - LAGRANGIAN INTERPOLATION; AN H $\mathrm{H}^{1}$-APPROXIMATION OF SCALAR FUNCTIONS}

In the examples of mixed and hybrid formulations presented in section 3 , an essential role is played by two Hilbert spaces : the space $H^{1}(\Omega)$ of scalar functions $v \in L^{2}(\Omega)$ such that $\operatorname{grad}_{\sim} v \in\left(L^{2}(\Omega)\right)^{\mathrm{n}}$ and the space $H(\operatorname{div} ; \Omega)$ of vector functions $q \in\left(L^{2}(\Omega)\right)^{n}$ such that $\operatorname{div} q \in L^{2}(\Omega)$. In this chapter we shall describe several choices of "finite-element-type" subspaces of $H^{1}(\Omega)$ and of $H(d i v ; \Omega)$.

For this general presentation we shall use the most elementary setting for finite element methods (some generalizations will be presented in section 7):

i) $\Omega$ will denote a bounded, open, polyhedral subset of $\mathbf{R}^{n}, \mathbf{n}=2$ or 3 ;

ii) $T_{h}$ will be a triangulation of $\bar{\Omega}$ by $n$ simplexes $T$ of diameter no greater than $h$, ( $T$ is a triangle for $n=2$, a tetrahedron for $n=3$ ), with

$$
\bar{\Omega}=\underset{T \in T_{h}}{U}
$$

for the definition of a triangulation. cf., CIARLET (1978, p.38) for example.The boundary of an $n$ simplex $T$, i.e. the union of its $n+1$ ( $n$-i)-dimensional faces, will be denoted by $\partial T$, and in a general manner we shall use the symbol $T^{\prime}$ to represent an arbitrary ( $n-1)$-dimensional face of $T$ and we shall write $T^{\prime}<T$; $T^{\prime}$ will be an edge of $T$ when $n=2$, a triangular face when $n=3$. We shall also use the following notation:

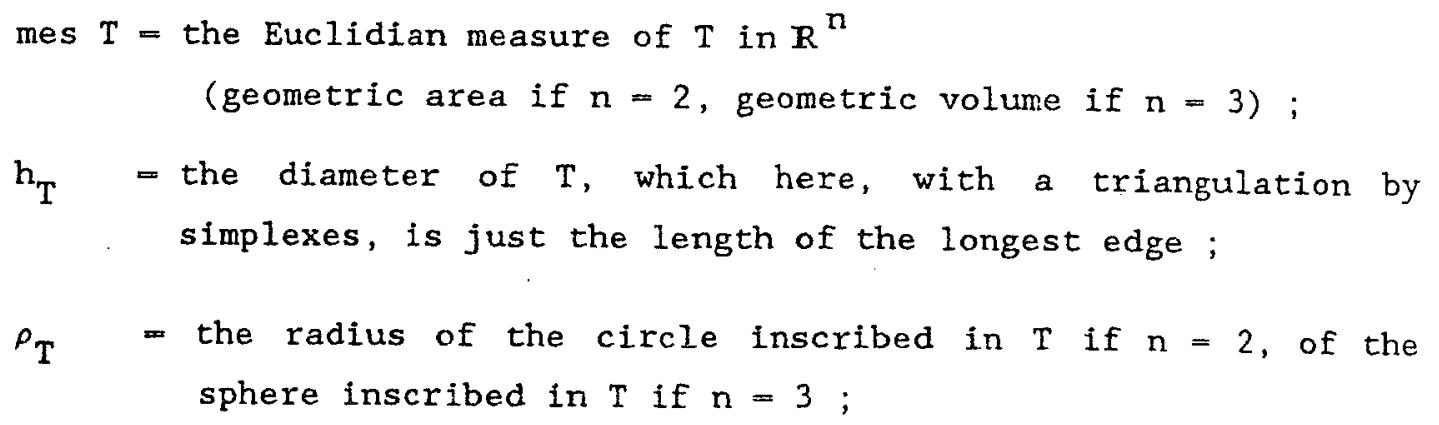


and

$$
\mathrm{h}=\max _{\mathrm{T} \in T_{\mathrm{h}}} \mathrm{h}_{\mathrm{T}}
$$

Let $H$ be a set of positive numbers. A family of triangulations $\left\{T_{h} ; \mathrm{h} \in H\right\}$ will be said to be regular if

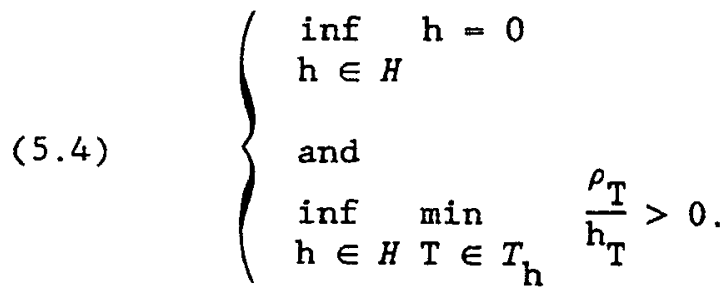

This definition is equivalent to that given by CIARLET (1978, p.124). We shall say that the family $\left\{T_{h} ; \mathrm{h} \in H\right\}$ is uniformly regular if

$$
\left\{\begin{array}{l}
\inf h=0 \\
h \in H \\
\text { and } \\
\inf _{h \in H}\left(\min _{\mathrm{T} \in T_{\mathrm{h}}} \rho_{\mathrm{T}}\right) / \underset{\mathrm{T} \in T_{\mathrm{h}}}{\left.\left(\max _{\mathrm{T}}\right)\right\}>0 .}
\end{array}\right.
$$

Thus a family of triangulations $\left\{T_{h} ; h \in H\right\}$ is uniformly regular if and only if it is regular and it satisfies the inverse hypothesis : there exists a constant $\mathrm{C}$, independent of $\mathrm{h}$, such that for each $\mathrm{h} \in H$

$$
\min _{\mathrm{T} \in T_{\mathrm{h}}} \mathrm{h}_{\mathrm{T}} \geq \mathrm{Ch},
$$

cf. CIARLET (1978, p.140).

Remark 5.1. The regularity hypothesis (5.4) is quite natural for the theory of interpolation by finite elements. However, in the chapters that follow we shall be rather abusive of the inverse hypothesis (5.6), using it often just to simplify 
the exposition of certain results.

For each natural number $k$, we denote by $P_{k}(T)$ the space of restrictions to $T$ of polynomial functions of degree $k$ in $n$ variables ; we have

$$
\operatorname{dim} P_{k}(T)=\frac{(n+k) !}{n ! k !}= \begin{cases}1 / 2(k+1)(k+2) & \text { if } n=2 \\ 1 / 6(k+1)(k+2)(k+3) & \text { if } n=3\end{cases}
$$

To an $n$ simplex $T$ and a positive integer $k$ is associated $\Sigma_{T}^{(k)}$, the principal lattice of order $k$ of the triangle $T$, i.e. the set of points of $T$ each of whose barycentric coordinates, relative to the vertices of $T$, is a multiple of $1 / k$. We have

$$
\operatorname{card} \Sigma_{T}^{(k)}=\frac{(n+k) !}{n ! k !}
$$

The Lagrangian interpolant $\mathcal{L}_{\mathrm{T}}^{(\mathrm{k})} \mathrm{v}$ on $\mathrm{T}$ of order $\mathrm{k}, \mathrm{k} \geq 1$, of a function $\mathrm{v} \in \mathrm{C}^{0}(\mathrm{~T})$ is by definition the unique function in $P_{k}(T)$ such that

$$
z_{T}^{(k)} v(a)=v(a)
$$

for all a $\in \Sigma_{\mathrm{T}}^{(\mathrm{k})}$.

Given a triangulation $T_{\mathrm{h}}$ of $\bar{\Omega}$ and a function $\mathrm{v} \in \mathrm{C}^{0}(\bar{\Omega})$, for a positive integer $\mathrm{k}$, we shall denote by $L_{h}^{(k)^{h}} v$ the function on $\bar{\Omega}$ which coincides with the polynomial function $\mathcal{L}_{T}^{(k)}\left(v_{T_{T}}\right)$ on $T$ for each simplex $T \in T_{h}$. One says that $\mathscr{L}_{h}^{(k)} \mathrm{V}$ is the Lagrangian interpolant of order $k$ on $T_{h}$ of the function $v$. It is essential to remark that $\mathcal{L}_{h}^{(k)} \mathrm{v}$ is, for each positive integer $k$, a continuous function on $\bar{\Omega}$. Thus one can easily verify that the mapping $\mathcal{L}_{h}^{(k)}$ is surjective from $c^{0}(\bar{\Omega})$ onto the space $\mathrm{L}_{\mathrm{h}}^{(k)}$ of simplicial Lagrangian interpolants defined by

$$
L_{h}^{(k)}=\left\{v \in C^{0}(\bar{\Omega}) ; \forall T \in T_{h}, \quad v_{T} \in P_{k}(T)\right\}
$$

For each $k, k \geq 1$, the space $L_{h}^{(k)}$ is a finite dimensional subspace of $H^{1}(\Omega)$. In dimensions $n=2$ and $n=3$ one may define $L_{T}^{(k)} v$ for $v \in H^{2}(T) \subset C^{0}(T)$ but not for $v \in H^{1}(T){ }^{(1)}$. With the aid of the following notation, given for each positive integer $\mathrm{m}$,

(1) We have adopted the notational convention $H^{m}(A)=H^{m}(A)$, i the interior of $A$. 


$$
\mathrm{H}^{\mathrm{m}}\left(T_{\mathrm{h}}\right)=\left\{\mathrm{v} \in \mathrm{L}^{2}(\Omega) ; \forall \mathrm{T} \in T_{\mathrm{h}},{ }^{\mathrm{v}} \mathrm{h}_{\mathrm{T}} \in \mathrm{H}^{\mathrm{m}}(\mathrm{T})\right\}
$$

one may characterize $L_{h}^{(k)}$ as being the fixed point space of the operator $\mathcal{L}_{h}^{(k)}$ on $H^{1}(\Omega) \cap H^{2}\left(T_{h}\right):$

$$
I_{h}^{(k)}=\left\{v \in H^{1}(\Omega) \cap H^{2}\left(T_{h}\right) ; v=L_{h}^{(k)} v\right\} .
$$

Thus we have

THEOREM 5.1. For each positive integer $\mathrm{k}$ and each triangulation $T_{\mathrm{h}}$ of $\bar{\Omega}$, the mapping $L_{h}^{(k)}$ defines a projection of $\mathrm{H}^{1}(\Omega) \cap \mathrm{H}^{2}\left(T_{h}\right)$ onto $\mathrm{L}_{h}^{(k)}$.

We also have

THEOREM 5.2. Suppose we are given a regular family $\left\{T_{\mathrm{h}} ; \mathrm{h} \in H\right\}$ of triangulations of $\bar{\Omega}$. For each positive integer $k$, there exists a constant $c$, independent of $h$, such that

$$
\begin{aligned}
& \left|v-L_{h}^{(k)} v\right|_{1, \Omega}+h^{-1}\left\|v-L_{h}^{(k)} v\right\|_{0, \Omega} \leq c h^{m}|v|_{m+1, \Omega} \\
& \qquad \text { for each } v \in H^{m+1}(\Omega), 1 \leq m \leq k .
\end{aligned}
$$

We shall not give here the demonstrations of these results fundamental for the study of the convergence of finite element methods but refer the reader to RAVIART \& THOMAS (1983, pp.79-103), for example, for the details of the proofs.

We would like now to make precise the idea of interpolation of the trace on the set $\partial T_{h}=\underset{T \in T_{h}}{U} \partial T$ of a scalar function $v$ continuous on $\bar{\Omega}$. For a triangulation $T_{h}$ of $\bar{\Omega}$ and a positive integer $k$, the space $L_{\partial T_{h}}^{(k)}$ is defined by

$$
\begin{aligned}
\mathrm{L}_{\partial T_{\mathrm{h}}}^{(\mathrm{k})}=\left\{\psi=\left(\psi_{\mathrm{T}}\right)_{\mathrm{T}} \in T_{\mathrm{h}} \in \underset{\mathrm{T} \in T_{\mathrm{h}}}{\Pi} \mathrm{L}^{2}(\partial \mathrm{T}) ; \exists \mathrm{v} \in \mathrm{L}_{\mathrm{h}}^{(\mathrm{k})}\right. \text { s.t. } \\
\left.\qquad \mathrm{T} \in T_{\mathrm{h}}, \mathrm{v}_{\mid \partial \mathrm{T}}=\psi_{\mathrm{T}}\right\} .
\end{aligned}
$$

The space $\mathrm{L}_{\partial T_{\mathrm{h}}}^{(\mathrm{k})}$ may also be characterized as being the space to which $\psi=\left(\psi_{\mathrm{T}}\right)_{\mathrm{T} \in T_{\mathrm{h}}}$ belongs if and only if it satisfies the following properties : 
i) $\psi_{T} \in P_{k}(\partial T) \quad$ for each $T \in T_{h}$

ii) $\psi_{T_{1}}=\psi_{T_{2}}$ on $T^{\prime}$

whenever $T^{\prime}$ is a face common to the simplexes $T_{1}$ and $T_{2}$ of $T_{h}$,

where $P_{k}(\partial T)$ is defined to be the space of restrictions to the boundary $\partial T$ of $T$ of polynomials of degree not greater than $k$ in $n$ variables or equivalently the space of functions in $n$ variables, polynomial of degree not greater than $k$ on each face $T^{\prime}$ of $T$ and continuous on $\partial T$. One may easily verify that

(5.16) $\operatorname{dim} P_{k}(\partial T)= \begin{cases}\operatorname{dim} P_{k}(T) & \text { if } k \leq n \\ \operatorname{dim} P_{k}(T)-\operatorname{dim} P_{k-n-1}(T) & \text { if } k>n .\end{cases}$

Let $\mathcal{L}_{\partial T_{\mathrm{h}}}^{(\mathrm{k})}$ be the mapping which associates to an element $\psi=\left(\psi_{\mathrm{T}}\right) \mathrm{T} \in T_{\mathrm{h}} \in \underset{\mathrm{T} \in T_{\mathrm{h}}}{\Pi} \mathrm{C}^{0}(\partial \mathrm{T})$ the element $\left(\mathcal{L}_{\partial \mathrm{T}}^{(\mathrm{k})} \psi_{\mathrm{T}}\right)_{\mathrm{T} \in T_{\mathrm{h}}}$, where $\mathcal{L}_{\partial \mathrm{T}}^{(\mathrm{k})} \psi_{\mathrm{T}}$, the $k^{\text {th }}$ order Lagrangian interpolant of

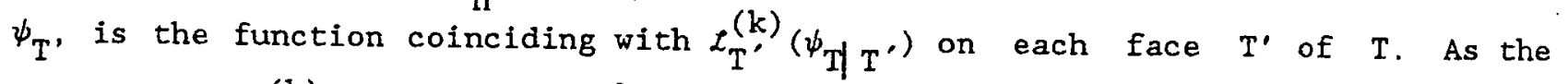
elements of $L_{h}^{(k)}$ are of class $c^{0}$ (cf. CIARLET (1978, p.95) for example), for each function $\mathrm{v} \in \mathrm{H}^{1}(\Omega) \cap \mathrm{H}^{2}\left(\partial T_{h}\right)$, we have

$$
\mathscr{L}_{\partial \mathrm{T}}^{(\mathrm{k})}\left(\mathrm{v}_{\mid \partial \mathrm{T}}\right)=\left(\mathcal{L}_{\mathrm{T}}^{(\mathrm{k})}\left(\mathrm{v}_{\mid \mathrm{T}}\right)\right) \mid \partial \mathrm{T} \quad \text { for all } \mathrm{T} \in T_{\mathrm{h}^{\prime}}
$$

or in other words, if we put

$$
\psi_{(v)}=\left(\psi(v) T^{\prime} T \in T_{h}=\left(v_{\mid} \partial T^{\prime} T \in T_{h}\right.\right.
$$

then we have

$$
\mathscr{L}_{\partial T_{h}^{(k)} \psi(v)}=\left(\left.\left(\mathcal{L}_{h}^{(k)} \mathrm{v}\right)\right|_{\partial \mathrm{T}}\right) \mathrm{T} \in T_{\mathrm{h}}
$$

THEOREM 5.3 . FOr each positive integer $\mathrm{k}$ and each triangulation $T_{\mathrm{h}}$ of $\pi$, the mapping $\mathrm{v} \rightarrow L_{\partial T_{\mathrm{h}}}^{(\mathrm{k})} \psi(\mathrm{v})$, with $\psi(\mathrm{v})$ given by $\left.(5.17)\right)$, is a surjection of $\mathrm{H}^{1}(\Omega) \cap \mathrm{H}^{2}\left(T_{\mathrm{h}}\right)$ onto $\mathrm{L}_{\partial T_{\mathrm{h}}}^{(\mathrm{k})}$. It is a bijection of $\mathrm{L}_{\mathrm{h}}^{(\mathrm{k})}$ onto $\mathrm{L}_{\partial T_{\mathrm{h}}}^{(\mathrm{k})}$ only in case $\mathrm{k} \leq \mathrm{n}$.

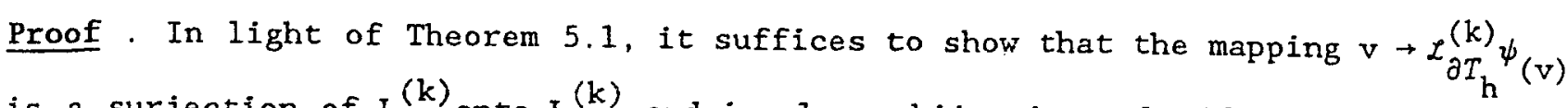
is a surjection of $\frac{(k)}{h}$ onto $L_{\partial T_{h}}^{(k)}$ and is also a bijection only if $k \leq n$. For $k \leq n$, the claim follows immediately from the facts that according to (5.16) the spaces $\mathrm{P}_{\mathrm{k}}(\mathrm{T})$ and $\mathrm{P}_{\mathrm{k}}(\partial \mathrm{T})$ have the same dimension and that for each $\psi, \psi=\left(\psi_{\mathrm{T}}\right)_{\mathrm{T} \in T_{\mathrm{h}}}$, 
belonging to $L_{\partial T_{h}}^{(k)}$, there exists a unique function $v$ in $L_{h}^{(k)}$ whose restriction to the boundary of $\mathrm{T}$ is equal to $\psi_{\mathrm{T}}$ for each $\mathrm{T}$ in $T_{h}$.

For $k>n$, the dimension of $P_{k}(T)$ is greater than that of $P_{k}(\partial T)$, so a bijection from $L_{h}^{(k)}$ onto $L_{\partial T_{h}}^{(k)}$ can not exist. To show surjectivity in this case, we have only to remark that given $\psi=\left(\phi_{T}\right)_{T \in T_{h}} \in L_{\partial T_{h}}^{(k)}$, the function $v \in L_{h}^{(k)}$, for example, defined by its values at the lattice points $a \in \underset{T \in T_{h}}{U} \Sigma_{T}^{(k)}$ as follows :

$$
v(a)= \begin{cases}\psi_{\mathrm{T}}(\mathrm{a}) & \text { if } a \in \partial \mathrm{T} \text { for some } \mathrm{T} \in T_{\mathrm{h}} \\ 0 & \text { otherwise }\end{cases}
$$

defines an antecedent of $\psi$.

Before announcing an error bound for interpolation in $L_{\partial T_{h}}^{(k)}$, we would like to define a norm on the space $\prod_{T \in T_{h}} H^{3 / 2}(\partial T)$ which will be seen to be well adapted to the analysis of dual hybrid methods. Thus, given a triangulation $T_{h}$ of $\bar{\Omega}$, we put

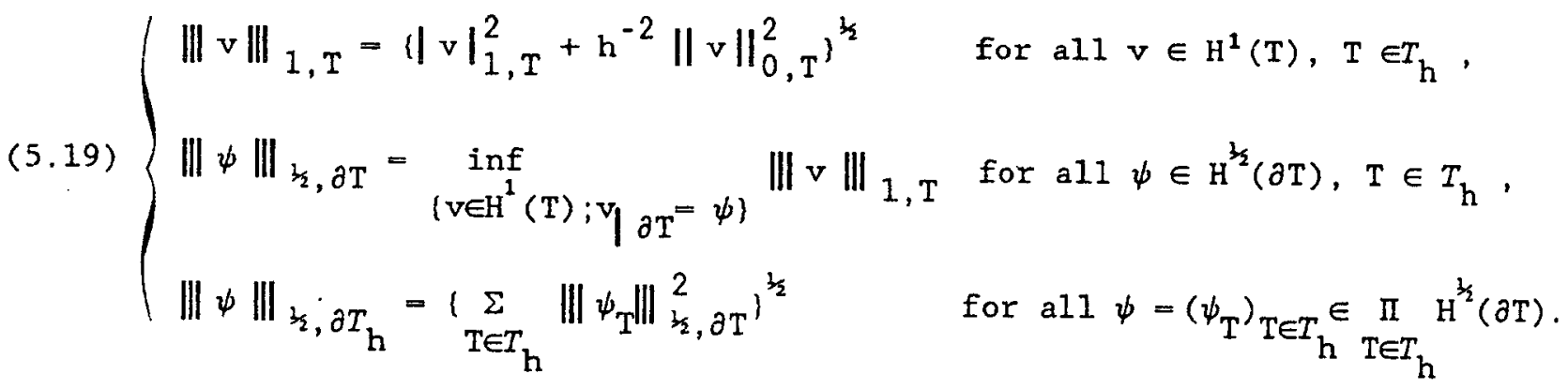

We may now state

THEORFM 5.4. Suppose $\left\{T_{\mathrm{h}} ; \mathrm{h} \in H\right\}$ is a regular family of triangulations of $\Omega$. For each positive integer $k$, there exists a constant $c$, indopendent of $h$, such that if $\mathrm{v} \in H^{\mathrm{m}+1}(\Omega), 1 \leq \mathrm{m} \leq \mathrm{k}$, and $\psi=\psi_{(\mathrm{v})}$ is determined by (5.17), then 


$$
\left\|\psi-\mathcal{L}_{\partial T_{\mathrm{h}}}^{(\mathrm{k})} \psi\right\| \|_{\xi_{\mathrm{h}}, \partial T_{\mathrm{h}}} \leq \mathrm{Ch} \mathrm{h}^{\mathrm{m}}|\mathrm{v}|_{\mathrm{m}+1, \Omega}
$$

Proof . With the norms defined in (5.19) we have

$$
\left\|\psi-L_{\partial T_{h}}^{(k)} \psi\right\|\left\|_{\xi, \partial T_{h}}^{2} \leq\right\| v-L_{h}^{(k)} v\left\|_{1, \Omega}^{2}+h^{-2}\right\| v-L_{h}^{(k)} v \|_{0, \Omega}^{2},
$$

so the demonstration is completed on applying Theorem 5.2.

\section{6 - INTERPOLATION OF THE MOMENTS OF A VECTORIAL FUNCTION ; AN H(div) APPROXIMATION OF VECTOR VALUED FUNCTIONS}

We retain here the assumptions made in Section 5 concerning the domain $\Omega \mathbf{R}^{n}$, $n=2$ or 3 , and the triangulation $T_{h}, \bar{\Omega}=\underset{T \in T_{h}}{U} T$. Given a positive integer $k$, we shall associate to each $n$-simplex $T \in T_{h}$ a subspace $D_{k}(T)$ of the product space $\left(P_{k}(T)\right)^{n}$ and an interpolation operator $E_{T}^{(k)}$ defined on a subspace $H(d i v ; T)$ of $\mathrm{H}(\mathrm{div} ; \mathrm{T})$ and having values in the space $\mathrm{D}_{\mathrm{k}}(\mathrm{T})$. Denoting by $H\left(\operatorname{div} ; T_{h}\right)$ the space defined by

$$
H\left(\operatorname{div} ; T_{\mathrm{h}}\right)=\left\{\mathrm{q} \in\left(\mathrm{L}^{2}(\Omega)\right)^{\mathrm{n}} ; \forall \mathrm{T} \in T_{\mathrm{h}}, \mathrm{q}_{\mathrm{T}} \in H(\operatorname{div} ; \mathrm{T})\right\}
$$
we can associate to each vector function $q \in H\left(\operatorname{div} ; T_{h}\right)$ the function $E_{h}^{(k)} q \in$
$\left(L^{2}(\Omega)\right)^{n}$ determined by

$$
\left(E_{\mathrm{h}}^{(\mathrm{k})} \underline{\mathrm{q}}\right) \mathrm{I}_{\mathrm{T}}=E_{\mathrm{T}}^{(\mathrm{k})}\left(\underline{\mathrm{q}}_{\mathrm{T}}\right)
$$

for all $T \in T_{\mathrm{h}}$.

The spaces $D_{k}(T)$ and the operators $E_{T}^{(k)}$ will be constructed in such a way that the following two hypotheses are satisfied

$$
\operatorname{div} \underline{q}=0 \Rightarrow \operatorname{div}\left(E_{\mathrm{T}}^{(\mathrm{k})} \mathrm{q}\right)=0
$$

for all $\mathrm{T} \in T_{\mathrm{h}}$, and for all qeH (div; $T$ ),

and

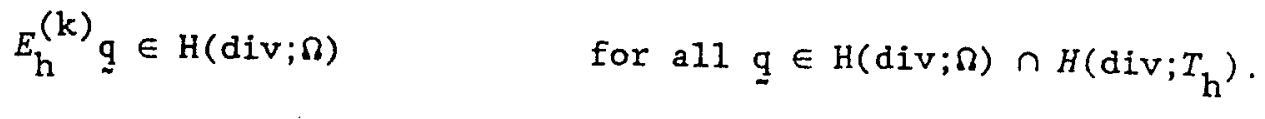

Property (6.3) is a local property of the interpolation of vector functions in $H(\operatorname{div} ; \mathrm{T})$. Its analogue for the approximation of scalar functions in $H^{1}(T)$ is that 
the interpolant of a function whose gradient vanishes also has a trivial gradient ; i.e., the interpolant of a constant function is a constant. However we did not think it necessary to state such a natural requirement.

Property (6.4) is a continuity condition for the interpolant of a vector function having a certain amount of regularity on $\bar{\Omega}$. Here of course the scalar analogue, that the interpolant $L_{h}^{(k)} v$ be in $H^{1}(\Omega)$ whenever $v \in H^{1}(\Omega) \cap H^{2}\left(T_{h}\right)$, is satisfied as the space $L_{h}^{(k)}$ is a subspace of $C^{0}(\bar{\Omega})$.

Remark 6.1 . Almost reflexively, a mathematician's first idea is to try to reduce the study of interpolation of vector functions to that of scalar functions as given in the preceeding section by choosing

$$
\left\{\begin{array}{l}
D_{k}(T)=\left(\mathrm{P}_{k}(\mathrm{~T})\right)^{\mathrm{n}}, \\
H(\mathrm{div}, \mathrm{T})=\left(\mathrm{H}^{2}(\mathrm{~T})\right)^{\mathrm{n}} \\
\frac{E_{\mathrm{T}}^{(\mathrm{k})}=\left(\mathcal{L}_{\mathrm{T}}^{(\mathrm{k})}\right) \otimes \mathrm{n}}{\mathrm{T}},
\end{array}\right.
$$

$\left(k^{\text {th }}\right.$ order Lagrangian interpolation on $\mathrm{T}$ carried out component by component).

One may check that condition (6.4) is satisfied; however, (6.3) is not. To see this consider in 2 dimensions, on the reference triangle $\hat{T}$ with vertices $(0,0)$, $(1,0)$ and $(0,1)$, the function $q=\left(q_{1}, q_{2}\right)$ given by

$$
\left\{\begin{array}{l}
q_{1}\left(x_{1}, x_{2}\right)=\frac{3}{2} x_{1}^{2}-x_{1} x_{2} \\
q_{2}\left(x_{1}, x_{2}\right)=3 / 2 x_{2}^{2}-x_{1} x_{2} .
\end{array}\right.
$$

This function $q$ is divergence free. Yet its Lagragian interpolant in $\left(P_{1}(T)\right)^{2}$ is none other than the function $\mathrm{x} \longrightarrow\left(\frac{1}{2} \mathrm{x}_{1}, \frac{1}{2} \mathrm{x}_{2}\right)$ in $\hat{\mathrm{T}}$ whose divergence is the constant function $\mathrm{x} \longrightarrow 1$.

We shall give two families of examples of $\mathrm{H}$ (div) approximation ; the first the original examples due to THOMAS (1977, Chapter III), cf. also RAVIART \& THOMAS (1977), for the 2 dimensionaI case and to NEDELEC (1980) for the 3 dimensional case, we present in some detail ; the second examples due to BREZZI, DOUGLAS, \& MARINI (1985) in the 2-dimensional case, and to BREZZI, DOUGLAS, DURAN, \& FORTIN (1987) in the 3-dimensional case will be described more briefly. Yet another family of examples of $H(d i v)$ approximation may be found in NEDELEC (1986). 
For each positive integer $k$ we put

$$
D_{k}=\left(P_{k-1}\right)^{n} \oplus \underset{\sim}{x-1}
$$

and define $D_{k}(T)$ to be the space of restrictions to $T$ of the functions of $D_{k}$. In other words, the vector function $q=\left(q_{1}, \ldots, q_{n}\right)$ belongs to $D_{k}(T)$ if and only if there exists $n+1$ scalar polynomial functions $q_{i}^{*} \in P_{k-1}(T), i=0,1, \ldots, n$, such that

$$
q_{i}(x)=q_{i}^{*}(x)+x_{i} q_{0}^{*}(x)
$$

for all $x \in T, i=1, \ldots, n$.

The expression (6.6) for $\mathrm{q}$ may be made unique by further requiring that $q_{0}^{*}$ be homogeneous of degree $k-1$. Thus we have

$$
\operatorname{dim} D_{k}(T)=(n+1) \operatorname{dim} P_{k-1}(T)-\operatorname{dim} P_{k-2}(T)
$$

which together with (5.7) gives us

$$
\operatorname{dim} D_{k}(T)=(n+k) \frac{(n+k-2) !}{(n-1) !(k-1) !},
$$

or more specifically

$$
\operatorname{dim} D_{k}(T)= \begin{cases}k(k+2), & \text { when } n=2, \\ \frac{1}{2} k(k+1)(k+3), & \text { when } n=3 .\end{cases}
$$

For each nonnegative integer $l$ we denote by $M_{\ell}(T)$ the space of linear forms on $\left(C^{0}(T)\right)^{n}$ defined by

$$
M_{\ell}(\mathrm{T})=\left\{\left(\underline{q} \rightarrow \int_{\mathrm{T}} \underline{\mathrm{q}} \cdot \underline{\mathrm{r}} \mathrm{dx}\right) \in \mathcal{L}\left(\left(\mathrm{C}^{0}(\mathrm{~T})\right)^{\mathrm{n}}, \mathbf{R}\right) ; \underset{\sim}{\mathrm{r}} \in\left(\mathrm{P}_{\ell}\right)^{\mathrm{n}}\right\}
$$

i.e. an element of $M_{\ell}(T)$ assigns to the vector function $q$ on $T$ one of its moments on $T$ of order no greater than $\ell$. In keeping with classical notation we shall write $M_{\ell}(T)=\{0\}$ for $\ell$ a negative integer, in particular for $\ell=-1$. Similarly for each nonnegative integer $\ell$ and each face $T^{\prime}$ of $T$ we denote by $M_{\ell}\left(T^{\prime}\right)$ the space of linear forms on $\left(\mathrm{C}^{0}(\mathrm{~T})\right)^{\mathrm{n}}$ defined by

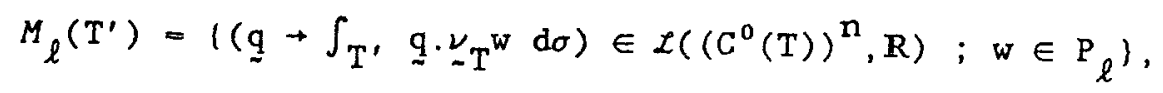

where $\nu_{T}$ is the unit exterior normal to $\partial T$, constant on each face $T^{\prime}$ of $T$. Thus an element of $M_{\ell}\left(T{ }^{\prime}\right)$ assigns to the vector function $q$ on $T$ one of the moments on $T$ ' of order no greater than $\ell$ of its exterieur normal trace $q \cdot \nu_{T}$. Now we define, for $k$ a positive integer, 
(6.10)

$$
M_{T}^{(k)}-\left(T^{\prime}<T=M_{k-1}\left(T^{\prime}\right)\right) \cup M_{k-2}(T)
$$

where $T^{\prime}<T$ mean $T^{\prime}$ is an (n-1)-dimensional face of $T$. Without change of notation we shall consider henceforth that the forms in $M_{\mathrm{T}}^{(k)}$ are defined on the space $H(\operatorname{div} ; \mathrm{T})$,

$$
H(\operatorname{div} ; \mathrm{T})=\left\{\underline{q} \in \mathrm{H}(\operatorname{div} ; \mathrm{T}) ; \mathrm{q} \cdot{ }_{\sim} \mathrm{T} \in \mathrm{L}^{2}(\partial \mathrm{T})\right\}
$$

The dimension of the space $M_{T}^{(k)}$ is given by

$$
\operatorname{dim} \frac{M}{T}=(\mathrm{k}) \mathrm{dim} \mathrm{P}_{\mathrm{k}-1}\left(\mathbf{R}^{\mathrm{n}-1}\right)+\mathrm{n} \operatorname{dim} \mathrm{P}_{\mathrm{k}-2}(\mathrm{~T})
$$

or more explicitly, by

$$
\operatorname{dim} M_{T}^{(k)}=(n+k) \frac{(n+k-2) !}{(n-1) !(k-1) !} .
$$

Thus we see that

$$
\operatorname{dim} D_{k}(T)=\operatorname{dim} M_{T}^{(k)}, \quad \quad k=1,2, \ldots
$$

THEOREM 6.1. Let $\mathrm{T}$ be an $\mathrm{n}$ simplex and $\mathrm{k} a$ positive integer. For each $q \in H(\operatorname{div} ; \mathrm{T})$ there exists one and only one function, which we shall denote $E_{\mathrm{T}}^{(\mathrm{k})} \mathrm{q}$, in $D_{k}(T)$ for which

$$
\mathrm{m}\left(E_{\mathrm{T}}^{(\mathrm{k})} \mathrm{q}\right)=\mathrm{m}(\mathrm{q})
$$$$
\text { for } a 11 \mathrm{~m} \in M_{\mathrm{T}}^{(\mathrm{k})} \text {. }
$$

The mapping $E_{\mathrm{T}}^{(\mathrm{k})}: H(\mathrm{div}, \mathrm{T}) \rightarrow \mathrm{D}_{\mathrm{k}}(\mathrm{T})$ thus defined further satisfies the commuting diagram property

i.e.

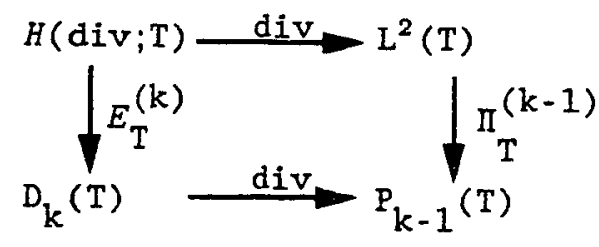

$(6.15)$

$$
\operatorname{div}\left(E_{\mathrm{T}}^{(k)} \mathrm{q}\right)=\Pi_{\mathrm{T}}^{(k-1)}(\operatorname{div} \mathrm{q})
$$

for all $\underline{\mathrm{q}} \in H(\operatorname{div} ; \mathrm{T})$,

where $\Pi_{T}^{(k-1)}$ denotes the orthogonal projection in $L^{2}(T)$ onto $P_{k-1}(T)$. 
Proof - Suppose for the moment that the operator $E_{\mathrm{T}}^{(\mathrm{k})}$ from $H(\mathrm{div} ; \mathrm{T})$ to $\mathrm{D}_{\mathrm{k}}(\mathrm{T})$ satisfying (6.14) is well defined and let $q \in H($ div; $T$ ). Then it is clear that the divergence of any element of $D_{k}(T)$, and in particular of $E_{T}^{(k)} q$, is a polynomial of degree not exceeding $k-1$. Thus using Green's formula with (6.14) we have

$$
\int_{T} \operatorname{div}\left(E_{T}^{(k)} q\right) w d x=\int_{T} \operatorname{div} q w d x \quad \text { for all } w \in P_{k-1}(T)
$$

or equivalently (6.15).

To show that given $\mathrm{q} \in H(\operatorname{div} ; \mathrm{T}),(6.14)$ does indeed determine a unique element of $D_{k}(T)$, in view of (6.13) it suffices to show that if $p \in D_{k}(T)$ and

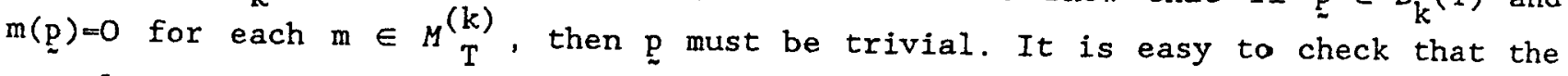
normal trace on any face $T^{\prime}$ of $T$ of any element of $D_{k}(T)$ is a polynomial of degree at most $k-1$. Thus, the normal trace of $p$ on $T$ must be trivial. The divergence of $\underset{\sim}{p}$ a priori a polynomial of degree at most $k-1$ on $T$, can then be shown to vanish by using Green's formula. It follows that $\underline{p}$ belongs to the subspace $\left(P_{k-1}(T)\right)^{n}$ of $D_{k}(T)$; to see this simply apply Euler's identity for homogeneous functions : grad $f(\underline{x}) \cdot x_{\sim}=\alpha f(x)$ whenever $f$ is homogeneous of degree $\alpha$. Next we show that if $\underline{\sim}^{\prime}$ is a vector normal to a face $T^{\prime}$ of $T$ that $\underset{\sim}{p} \underline{\sim}^{\prime}$ vanishes on $T$. As $\underset{\sim}{p} \nu^{\prime}$ vanishes on $T^{\prime}$, we have that $\underline{p} \cdot \underline{\nu}^{\prime}=b w$, where $b$ is the first degree polynomial whose zeroes form the $(n-1)$-dimensional hyperplane containing $T^{\prime}$, and $w \in P_{k-2}$. Now $w_{\sim}^{\prime} \in\left(P_{k-2}\right)^{n}$
and we have

$$
\int_{T} \underset{\sim}{p} \cdot\left(\underset{\sim}{\nu^{\prime}}\right) d x=\int_{T} b w w d x=0
$$

As $\mathrm{b}$ is either always positive or always negative on $T, w$ and hence $\underline{p}^{*}$ vanish on $T$. The theorem now follows on noting that for any $n$ of the $n+1$ faces of $T$, the corresponding normal vectors form a basis for $\mathbf{R}^{n}$.

For each $T \in T_{h}$ we have now constructed an interpolation operator $E_{T}^{(k)}$ from $H($ div; $\mathrm{T})$ into $\mathrm{D}_{\mathrm{k}}(\mathrm{T}) \subset\left(\mathrm{P}_{\mathrm{k}}(\mathrm{T})\right)^{\mathrm{n}}$ satisfying (6.15) and a fortiori (6.3). To a triangulation $T_{h}$ of $\bar{\Omega}$ we associate the space $H\left(\operatorname{div} ; T_{h}\right)$ defined by (6.1) together with (6.11):

$$
\begin{aligned}
& H\left(\operatorname{div} ; T_{\mathrm{h}}\right)=\left\{\underline{q} \in\left(\mathrm{L}^{2}(\Omega)\right)^{\mathrm{n}} ; \forall \mathrm{T} \in T_{\mathrm{h}},\right. \\
&\left.\quad \mathrm{q}_{\mathrm{T}} \in \mathrm{H}(\operatorname{div} ; \mathrm{T}) \text { and } \underline{q}_{\mathrm{T}} \cdot \nu_{-T} \in \mathrm{L}^{2}(\partial \mathrm{T})\right\}
\end{aligned}
$$

and the equilibrium interpolation operator $E_{\mathrm{h}}^{(\mathrm{k})}$ of order $\mathrm{k}$ on $H\left(\mathrm{div} ; T_{\mathrm{h}}\right.$ ) constructed from the operators $E_{T}^{(k)}$ with the aid of $(6.2)$. 
To see that $E_{\mathrm{h}}^{(\mathrm{k})}$ satisfies $(6.4)$, we note that if $\mathrm{q}$ is sufficiently regular in the sense that $\mathrm{q} \in \mathrm{H}(\operatorname{div} ; \Omega) \cap H\left(\operatorname{div} ; T_{\mathrm{h}}\right)$ then Theorem 1.3 implies

$$
\begin{array}{ll}
q \mid T_{1} \cdot \nu T_{1}+q & q_{2} \cdot \nu T_{2}=0 \quad \text { on } T^{\prime}, \text { for all } T^{\prime}=T_{1} \cap T_{2} \\
& \text { with } T_{1}, T_{2} \in T_{h} .
\end{array}
$$

Thus, for each $T^{\prime}=T_{1} \cap T_{2}$ with $T_{1}, T_{2} \in T_{h^{\prime}} \underline{r}=E_{h}^{(k)} q$ satisfies

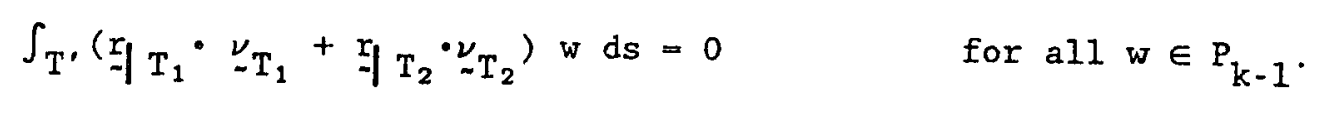

The restrictions $y_{T} \in D_{k}(T), T \in T_{h}$, are polynomial of degree at most $k$ and their normal traces on $\partial T$ are piecewise polynomial of degree at most $k-1$; indeed, on each face $T^{\prime}$ of $T, T \in T_{h}, \underset{\sim}{x} \sim_{\sim} T$ is constant. With this observation of central importance we see that $\underset{\sim}{r}$ satisfies the continuity condition $(6.17)$ and hence $\underline{r}=E_{\mathrm{h}}^{(\mathrm{k})} \mathrm{q} \in \mathrm{H}(\mathrm{div} ; \Omega) ;$ i.e. $(6.4)$ holds.

We denote by $\mathrm{E}_{\mathrm{h}}^{(\mathrm{k})}$ the finite dimensional space of equilibrium interpolants

$$
E_{h}^{(k)}=\left\{q \in H(\operatorname{div} ; \Omega) ; \forall T \in T_{h}, \underset{q_{T}}{q} \in \underset{\sim k}{D_{k}}(T)\right),
$$

which, as we have just seen, is the space of functions $q \in\left(L^{2}(\Omega)\right)^{n}$ for which the restriction $q_{T}$ may be identified with a function in $D_{k}(T)$ for each $T \in T_{h}$ and for which (6.17) holds. In analogy with (5.12), $\mathrm{E}_{\mathrm{h}}^{(\mathrm{k})}$ may also be characterized as a space of fixed points as follows :

$(6.18$ bis $) \quad E_{h}^{(k)}=\left(q \in H(\operatorname{div} ; \Omega) \cap H\left(\operatorname{div} ; T_{h}\right) ; E_{h}^{(k)}(q)=q\right)$.

Thus we have

THEOREM 6.2 . For each positive integer $\mathrm{k}$, and each triangulation $T_{\mathrm{h}}$ of $\bar{\Omega}$, the mapping $E_{h}^{(k)}$ is a projection from $\mathrm{H}(\operatorname{div} ; \Omega) \cap H\left(\operatorname{div} ; T_{h}\right)$ onto $\mathrm{E}_{\mathrm{h}}^{(\mathrm{k})}$.

The next theorem gives a bound for the error of interpolation by $E_{h}^{(k)}$ :

THEOREM 6.3. Suppose $\left\{T_{\mathrm{h}} ; \mathrm{h} \in H\right\}$ is a regular family of triangulations of $\bar{\Omega}$ and $\mathrm{k}$ is a positive integer. There exists a constant $\mathrm{C}$, independent of $\mathrm{h}$, such that 


$$
\left\|\mathrm{q}-E_{\mathrm{h}}^{(\mathrm{k})} \mathrm{q}\right\|_{0, \Omega} \leq \mathrm{Ch}^{\ell}|\mathrm{q}|_{\ell, \Omega}
$$

for all $\mathrm{q} \in\left(\mathrm{H}^{\ell}(\Omega)\right)^{\mathrm{n}}, 1 \leq \ell \leq \mathrm{k}$,

and

$$
\left\|\operatorname{div}\left(\underline{q}-E_{h}^{(k)} q\right)\right\|_{O, \Omega} \leq \mathrm{Ch}^{\ell}|\operatorname{div} q|_{\ell, \Omega}
$$

for all $\mathrm{q} \in\left(\mathrm{H}^{1}(\Omega)\right)^{\mathrm{n}}$ with $\operatorname{div} \underline{q} \in \bar{H}^{\ell}(\Omega), 0 \leq l \leq k$.

Proof Estimate (6.20) is a classical corollary of property (6.15) identifying $\operatorname{div}\left(E_{T}^{(k)} q\right)$ as the image under the orthogonal $L^{2}$-projection onto $L_{h}^{(k)}$ of $\operatorname{div} q$, and we shall not give the details of its demonstration. The argument justifying (6.19) is more technical : for each $T \in T_{h}$ we must find a bound for the quantity $\left\|q-E_{T}^{(k)} q\right\|_{O, T}=\left[\sum_{i=1}^{n} \| q_{i}-\left(E_{T}^{(k)} q_{i}\right)_{0, T}\right]^{2 / 5}$; however, this can not be done component by component as the interpolation space is not a simple tensor product of $n$ copies of the same space of polynomials. Thus it is necessary to work directly with the subspaces of vector functions of $n$ variables with values in $\mathbb{R}^{n}$. We employ the technique of reducing the question of the error bound on $T$ to that on the reference triangle $\hat{\mathrm{T}}$ as in CIARLET \& RAVIART (1972). For each $T \in T_{h}$, there exists an affine transformation $F: \hat{T} \rightarrow T=F(\hat{T})$, bijective from $\hat{T}$ onto $T$. We denote by $D F$ the linear tangent mapping and by $J$ the Jacobian of the transformation. We have here $|\mathrm{J}|=$ (mes $\mathrm{T}$ )/(mes $\hat{\mathrm{T}}$ ). To each scalar function $\hat{v} \in$ $\mathrm{H}^{1}(\hat{\mathrm{T}})$, classically is associated the function $\mathrm{v} \in \mathrm{H}^{1}(\mathrm{~T})$, defined by

$$
v(x)=\vartheta(x)
$$

for each $x=F(\hat{x}) \in T$.

Here, to each vector function $\hat{q} \in H(d i v ; \hat{T})$ we associate the function $q$, that we shall show belongs to $\mathrm{H}(\operatorname{div} ; \mathrm{T})$, defined by

$$
q(x)=\frac{1}{|J|} D F \underline{q}(\hat{x})
$$

for each $x=F(\hat{x}) \in T$.

The transformation (6.22) was constructed in such a way that

$$
\int_{T} q \cdot \operatorname{grad} v \mathrm{v} x=\int_{\hat{T}} \underset{\sim}{q} \cdot \operatorname{grad} \hat{\sim} \hat{v} \mathrm{~d} .
$$

Using Green's formula we deduce, cf. THOMAS (1976, Lemma 5.1), that

$$
\int_{T} v \operatorname{div} q d x=\int_{\hat{T}} \hat{v} \operatorname{div} \hat{q} d \hat{x}
$$


and we have, with $\hat{\sim}$ denoting of course the exterieur unit normal of $\partial \hat{\mathrm{T}}$,

$$
\int_{\partial \mathrm{T}} v \underline{q} \cdot \underline{\nu} \mathrm{d} \sigma=\int_{\partial \hat{\mathrm{T}}} \hat{\theta} \underline{\hat{q}} \cdot \hat{\underline{\nu}} \mathrm{d} \hat{\sigma}
$$

for all sufficiently regular functions $v$ and $q$. Thus $q \longrightarrow q$ is a bijective affine transformation from $\mathrm{H}(\operatorname{div} ; \hat{\mathrm{T}})$ onto $\mathrm{H}(\mathrm{div} ; \mathrm{T})$ such that

$$
\text { (mes } \mathrm{T}) \operatorname{div} \underline{q}=(\operatorname{mes} \hat{\mathrm{T}}) \operatorname{div} \underline{q}
$$

and on each face $T^{\prime}=F\left(\hat{T}^{\prime}\right)$ of $T$

$$
\left(\operatorname{mes} \mathrm{T}^{\prime}\right) \mathrm{q} \cdot \nu=\left(\operatorname{mes} \hat{\mathrm{T}}^{\prime}\right) \underset{\boldsymbol{q}}{\mathrm{q}} \cdot \hat{\nu}
$$

Furthermore, it is immediately verified that this transformation takes $D_{k}(\hat{T})$ onto $\mathrm{D}_{k}(\mathrm{~T}):$

$$
\underline{q} \in D_{k}(T) \Leftrightarrow \hat{q} \in D_{k}(\hat{T})
$$

We conclude from (6.27) and (6.28) that the interpolant in $D_{k}(T)$ of a function $q$, image of the function $\underline{q}$ defined on $\hat{T}$, is none other than the image of the interpolant in $D_{k}(\hat{T})$ of $\hat{q}$ :

$$
\left(E_{\mathrm{T}}^{(\mathrm{k})} \mathrm{q}\right) \cdot \mathrm{F}=\frac{1}{|\mathrm{~J}|} \mathrm{DF}\left(E_{\hat{\mathrm{T}}}^{(\mathrm{k})} \stackrel{\mathrm{q}}{\mathrm{L}}\right) .
$$

This property allows us to reduce the problem of obtaining an error bound for interpolation on $\mathrm{T}$ to that of obtaining an error bound for interpolation on $\hat{\mathrm{T}}$, the reference n-simplex. As the subspace $\left(P_{k-1}\right)^{n}$ of $D_{k}$ is invariant under the interpolation operator, we know that there exists a constant $\hat{C}$ depending only on $\hat{T}$ and $k$ such that.

$$
\left\|\underline{q}-E_{\hat{\mathrm{T}}}^{(\mathrm{k})} \hat{\mathrm{q}}\right\|_{O, \hat{\mathrm{T}}} \leq \hat{\mathrm{C}}|\underline{q}|_{\ell, \hat{\mathrm{T}}}
$$

$$
\begin{aligned}
& \text { for all } \underline{\underline{q}} \in\left(H^{\ell}(\hat{\mathrm{T}})\right)^{\mathrm{n}} \text { with } \\
& 1 \leq \ell \leq k
\end{aligned}
$$

With this bound and change of variable formulas we obtain for each $T$ in $T_{h}$ a local bound for $\left\|\mathrm{q}-E_{\mathrm{T}}^{(\mathrm{k})} \mathrm{q}\right\|_{\mathrm{O}, \mathrm{T}}$ and then the global bound (6.19).

Remark 6.2 . The introduction of the spaces $D_{k}$ is fundamental for our construction of an $\mathrm{H}(\mathrm{div} ; \Omega)$ approximation. We have the strict inclusions $\left(\mathrm{P}_{k-1}\right)^{n}$ $\subset D_{k} \subset\left(P_{k}\right)^{n}$, and we have chosen to index by $k$ the space $D_{k}$ strictly between $\left(P_{k-1}\right)^{n}$ and $\left(P_{k}\right)^{n}$ though in the literature one also finds this same space indexed $\therefore \quad:-1$. Our choice was made with consideration for the error bounds. As Lagrangian 
interpolation, $\mathcal{L}_{h}^{(k)}$, by functions locally in $\mathrm{P}_{k}$ leads to error bounds in $\mathrm{H}^{1}(\Omega)$ of order $h^{k}$, so the interpolation $E_{h}^{(k)}$, with $E$ as in equilibrium, by functions locally in $D_{k}$ leads to an error in $H(d i v ; \Omega)$ of order $h^{k}$.

Remark 6.3. The transformation $q \longrightarrow q$ introduced during the demonstration of Theorem 6.3 in (6.22) is none other than the transformation known in mechanics as the Piola transformation of a vector field defined on a manifold of $\mathbf{R}^{n}$, cf. for example MARSDEN \& HUGHES (1983, pp 116-118).

Next we present a few results concerning the approximation of the normal traces on the set $\partial T_{h}=\underset{T \in T_{h}}{U} \partial T$ of sufficiently regular vector functions $q$, for example $q \in\left(H^{1}(\Omega)\right)^{n}$. Given a triangulation $T_{h}$ of $\bar{\delta}$ and a positive integer $k$, we denote by $\mathrm{E}_{\partial T_{\mathrm{h}}}^{(\mathrm{k})}$ the space defined by

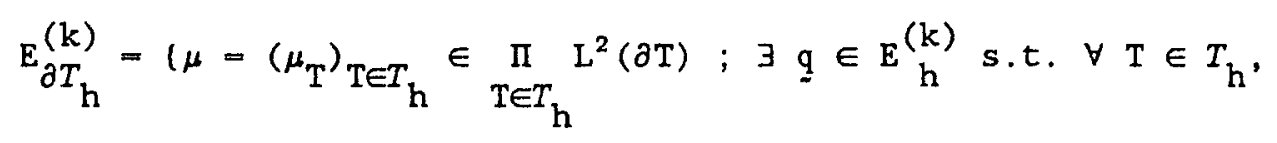

$$
\left.\underline{q} \mid \partial \mathrm{T}^{\bullet} \nu_{\mathrm{T}}=\mu_{\mathrm{T}}\right\}
$$

where $\frac{E}{h}$ (k) is the space defined by (6.18). The space $E_{\partial T_{h}}^{(k)}$ may also be characterized as the space of functions $\mu=\left(\mu_{T}\right)_{T \in T}$ satisfying the following two properties :

$$
\begin{cases}\text { i) } \mu_{T} \in D_{k}(\partial T), & \text { for each } T \in T_{h} \\ \text { and } & \\ \text { ii) } \mu_{T_{1}}+\mu_{T_{2}}=0 & \text { on each } T^{\prime}, T^{\prime}=T_{1} \cap T_{2} \text { with } T_{1}, T_{2} \in T_{h^{\prime}},\end{cases}
$$

where by $D_{k}(\partial T)$ we mean the space

$$
D_{k}(\partial T)=\left\{\mu \in L^{2}(\partial T) ; \exists \underline{q} \in D_{k}(T), \underline{q} \underline{\nu}_{-T}=\mu i\right.
$$

or equivalently the space of functions polynomial of degree at most $(k-1)$ on each face $T$ ' of $T$ with no continuity constraint at the intersection of two faces. Thus we have

$$
\operatorname{dim} D_{k}(\partial T)=(n+1) \frac{(n+k-2) !}{(n-1) !(k-1) !}
$$


Let $E_{\partial T_{h}}^{(k)}$ be the operator which associates to each element $\mu-\left(\mu_{T}\right) T \in T_{h} \in \underset{T \in T_{h}}{ } L^{2}(\partial T)$ the element $\left(E_{\partial \mathrm{T}}^{(\mathrm{k})} \mu_{\mathrm{T}}\right) \mathrm{T} \in T_{\mathrm{h}}$ where $E_{\partial \mathrm{T}}^{(\mathrm{k})}$ is the orthogonal projection from $\mathrm{L}^{2}(\partial \mathrm{T})$ onto $\mathrm{D}_{\mathrm{k}}(\partial \mathrm{T}), \mathrm{T} \in T_{\mathrm{h}}$. Associating to each vector function $\underline{q} \in H\left(\operatorname{div} ; T_{\mathrm{h}}\right) \cap \mathrm{H}(\operatorname{div} ; \Omega)$ the element

$$
{ }^{\mu}(\underline{q})=\left(\underline{q} \cdot \underline{\nu}^{T}\right){ }_{T} \in T_{h},
$$

we clearly have

$$
E_{\partial T_{h}}^{(k)} \mu_{(q)}=\left(\left(E_{h}^{(k)} \underline{q}\right) \cdot \nu_{\nu T}\right)_{T \in T} \in E_{\partial T h}^{(k)} .
$$

Hence we deduce :

THEOREM 6.4 For each positive integer $\mathrm{k}$ and each triangulation $T_{\mathrm{h}}$ of $\bar{n}$, the mapping q $\longrightarrow E_{\partial T_{\mathrm{h}}}^{(\mathrm{k})}{ }^{\mu}(\mathrm{q})$, with $\mu_{(\mathrm{q})}$ given by $(6.34)$, is a surjection of $\mathrm{H}(\operatorname{div} ; \Omega) \cap$ $H\left(\right.$ div; $T_{\mathrm{h}}$ ) onto $\mathrm{E}_{\partial T_{\mathrm{h}}}^{(\mathrm{k})}$. It is a bijection of $\mathrm{E}_{\mathrm{h}}^{(\mathrm{k})}$ onto $\mathrm{E}_{\partial T_{\mathrm{h}}}^{(\mathrm{k})}$ only in case $\mathrm{k}=1$.

Here we shall give only one result concerning error bounds for interpolation in $E_{\partial T_{h}}^{(k)}$. However, beforehand, in a manner analogous to (5.19), we define several norms that we shall find useful. Given a triangulation $T_{h}$ of $\bar{\Omega}$, we put

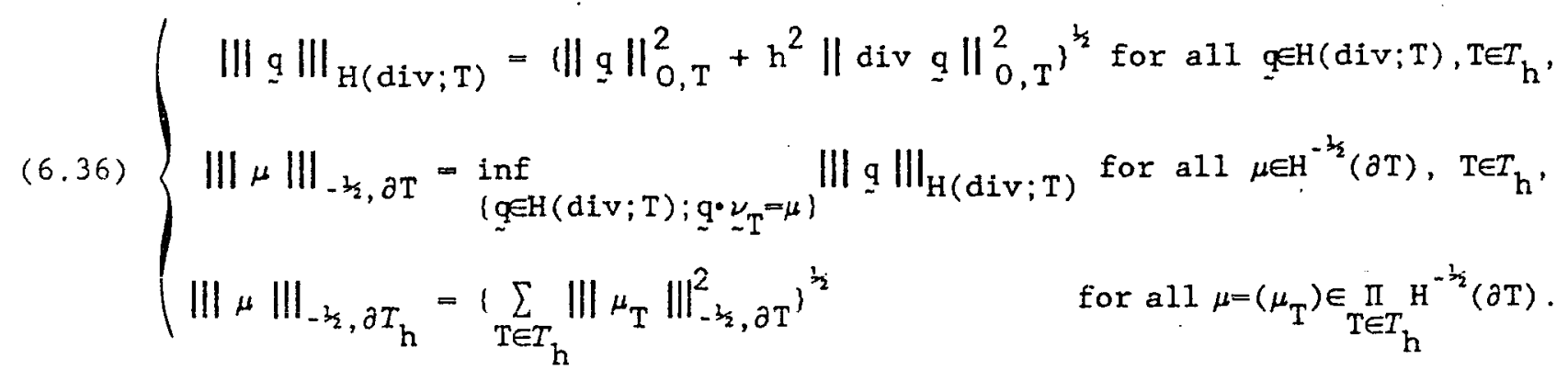

The following result will be needed for our analysis of primal hybrid methods :

THEOREM 6.5 Suppose $\left\{T_{\mathrm{h}} ; \mathrm{h} \in H\right\}$ is a regular family of triangulations of $\bar{n}$. For each positive integrer $\mathrm{k}$, there exists a constant $\mathrm{C}$, independent of $\mathrm{h}$, such that if $\mathrm{q} \in\left(\mathrm{H}^{\ell}(\Omega)\right)^{\mathrm{n}}, 1 \leq \ell \leq \mathrm{k}$, and $\mu=\mu(\mathrm{q})$ is determined by $(6.34)$, then 
(6.37)

$$
|I| q-\left.E_{\partial T_{h}}^{(\mathrm{k})} \mu||\right|_{-k_{2}, \partial T_{\mathrm{h}}} \leq \mathrm{Ch}^{\ell}|\mathrm{q}|_{\ell, \Omega} .
$$

To obtain this result we employ the bound (6.19) of order $\ell$ and the bound (6.20) of order $(\ell-1)$.

The second family of examples, is defined for integers $k, k \geq 2$. One chooses for interpolation space on the simplex $T$ the space

$$
D_{k}^{*}(T)=\left(P_{k-1}(T)\right)^{n}
$$

where we have kept the indexing convention of Remark 6.2 instead of that of BREZZI, DOUGLAS, \& MARINI (1985). For $q \in H\left(\right.$ div; $T$ ) the interpolant $E_{T}^{*(k)} q$ is defined to be the unique element of $D_{k}^{*}(T)$ such that

$$
\mathrm{m}\left(E_{\mathrm{T}}^{*(\mathrm{k})} \mathrm{q}\right)=\mathrm{m}(\underline{q}) \quad \text { for all } \mathrm{m} \in M_{\mathrm{T}}^{*(\mathrm{k})}
$$

where the set of moments $M_{T}^{*(k)}$ used to define the interpolant is the union

$$
\left.M_{T}^{*(k)}=\operatorname{T}_{T^{\prime}<T}^{(U} M_{(k-1)}\left(T^{\prime}\right)\right) \cup M \underset{k-2}{\operatorname{grad}}(T) \cup M_{k-1}^{\perp}(T)
$$

with $M_{\ell}\left(T^{\prime}\right)$ defined by $(6.9)$,

$$
M_{\ell}^{\operatorname{grad}}(T)=\left\{\left(\underline{q} \longrightarrow \int_{T} \underline{q} \cdot \operatorname{grad} w d x\right) \in \mathcal{L}\left(\left(C^{0}(T)\right)^{n}, R\right) ; w \in P_{\ell}\right\}
$$

and

$$
\begin{aligned}
& M_{\ell}^{\perp}(\mathrm{T})=\left\{\left(\underline{\mathrm{q}} \longrightarrow \int_{\mathrm{T}} \underline{\mathrm{q}} \cdot \underline{w} \mathrm{dx} \in \mathcal{L}\left(\left(\mathrm{C}^{0}(\mathrm{~T})\right)^{\mathrm{n}}, \mathrm{R}\right) ; \underset{\sim}{\mathrm{w}} \in\left(\mathrm{P}_{\ell}\right)^{\mathrm{n}},\right.\right. \\
& \underline{w} \cdot \nu_{-T}=0 \text { on } \partial T \text {, and } \operatorname{div} \underline{w}=0 \text { ). }
\end{aligned}
$$

Define the operator $E_{h}^{*(k)}$ by $(6.2)$ and the space $E_{h}^{*(k)}$ by analogy with (6.18) or equivalently $\left(6.18\right.$ bis). It can be shown that $(6.15)$ still holds with $E_{\mathrm{T}}^{(\mathrm{k})}$ replaced by $E_{\mathrm{T}}^{*(k)}$ and $\Pi_{\mathrm{T}}^{(\mathrm{k}-1)}$ replaced by $\Pi_{\mathrm{T}}^{(\mathrm{k}-2)}$, and thus so do $(6.3)$ and $(6.4)$ if $E_{\mathrm{h}}^{(\mathrm{k})}$ is also replaced by $E_{\mathrm{h}}^{*}(\mathrm{k})$. In fact the analogues of Theorem 6.1 and Theorem 6.2 obtained by replacing $E_{\mathrm{T}}^{(\mathrm{k})}, E_{\mathrm{h}}^{(\mathrm{k})}, \mathrm{D}_{\mathrm{k}}(\mathrm{T})$, and $\mathrm{E}_{\mathrm{h}}^{(\mathrm{k})}$ by $E_{\mathrm{T}}^{*(\mathrm{k})}, E_{\mathrm{h}}^{*(\mathrm{k})}, \mathrm{D}_{\mathrm{k}}^{*}(\mathrm{~T})$ and $E_{\mathrm{h}}^{*(\mathrm{k})}$ respectively remain valid. One can also demonstrate the following analogue of Theorem $6.3:$ if $\left\{T_{h} ; h \in H\right\}$ is a regular family of triangulations of $\bar{\Omega}$, then 


$$
\left\|\underline{q}-E_{h}^{*(k)} q\right\|_{0, \Omega} \leq \mathrm{Ch}^{\ell}|\mathrm{q}|_{\ell, \Omega}
$$

for all $\mathrm{q} \in\left(\mathrm{H}^{\ell}(\Omega)\right)^{\mathrm{n}}$ with

$1 \leq \ell \leq k$,

and

$$
\left\|\operatorname{div}\left(q-E_{h}^{*(k)} q\right)\right\|_{0, \Omega} \leq \mathrm{Ch}^{\ell}|\operatorname{div} q|_{\ell, \Omega}
$$

for all $q \in\left(H^{1}(\Omega)\right)^{n}$ with $\operatorname{div} \mathrm{q} \in \mathrm{H}^{\ell}(\Omega)$ for

$0 \leq \ell \leq \mathrm{k}-1$.

$\underline{\text { Remark 6.4 }}$. The space $\mathrm{E}_{\mathrm{h}}^{*(\mathrm{k})}$,

$$
\mathrm{E}_{\mathrm{h}}^{*(\mathrm{k})}=\left(\underline{\mathrm{q}} \in \mathrm{H}(\operatorname{div} ; \Omega) \cap H\left(\operatorname{div} ; T_{\mathrm{h}}\right) ; \underline{\mathrm{q}}=E_{\mathrm{h}}^{*(\mathrm{k})} \mathrm{q}\right) \text {, }
$$

is clearly a proper subspace of $E_{h}^{(k)}$. Hence fewer degrees of freedom are needed to determine the interpolants in $\mathrm{E}_{\mathrm{h}}^{*(\mathrm{k})}$ than in $\mathrm{E}_{\mathrm{h}}^{(\mathrm{k})}$. The interpolation errors in these two approximation spaces are of the same order when measured in the $L^{2}$-norm \|\|$_{O, \Omega}$; however, interpolation in $E_{h}^{*(k)}$ is less precise by one order than that in $\mathrm{E}_{\mathrm{h}}^{(\mathrm{k})}$ when measured in the $\mathrm{H}(\mathrm{div} ; \Omega)$ norm unless $|\operatorname{div} \mathrm{q}|_{\mathrm{k}-1, \Omega}=0$, i.e. unless div $q \in P_{k-2}$. Moreover one may verify that

$$
D_{k}^{*}(T)=\left\{\underline{q} \in D_{k}(T) ; \operatorname{div} \underline{g} \in P_{k-2}(T)\right\}
$$

Remark 6.5 . In fact one of the most important distinctions between the approximations in $\mathrm{E}_{\mathrm{h}}^{(\mathrm{k})}$, and in $\mathrm{E}^{*} \mathrm{~h}$ ) lies not in the interpolations themselves but in the fact that for the dual mixed method for the elliptic problem the $E_{h}^{(k)}$ interpolation for the vector variable is used in conjuntion with a locally $P_{k-1}$ interpolation for the scalar variable whereas when interpolation in $E_{h}^{*(k)}$ is used for the vector variable the natural domain for approximating the scalar variable is the space of locally $\mathrm{p}_{\mathrm{k}-2}$ functions. Thus; for example; when a piecewise constant approximation is sufficient for the scalar variable but more precision than that provided by the $\mathrm{E}_{\mathrm{h}}^{(1)}$ interpolant is desired for the vector variable, one has the option of approximating the vector function in $\mathrm{E}_{\mathrm{h}}^{*(2)}$ while retaining the locally $P_{0}$ approximation of the scalar function instead of using the full $P_{1}, E_{h}^{(2)}$ approximation. The gain in accuracy thus obtained, however, is, a priori, only in the $\left(L^{2}(\Omega)\right)^{n}$ norm. 
Using the families of examples of finite element type approximation developed in Sections 5 and 6 , we can construct approximations of order $k$, for each positive integer $k$, of functions in $H^{1}(\Omega)$ and of functions in $H(d i v ; \Omega)$ in the academic setting $\Omega$ a polyhedral domain triangulated by $n$-simplexes. To simplify vocabulary we fix $n=2$. Thus for the preceeding theory $\Omega$ was supposed to be an open, polygonal domain triangulated by triangles. Here we shall indicate a few generalizations related to the geometry of the triangulation : rectangular finite elements, curved finite elements such as curvilinear triangles, convex quadrilaterals...

We begin with the case of rectangular finite elements. We shall describe families of examples of approximation of functions in $H^{1}(\Omega)$ and of functions in $H(\operatorname{div} ; \Omega)$ when $\Omega$ is an open set of $R^{2}$ "triangulated" in the form $\Omega=\underset{T \in T}{U} \mathrm{~T}$ by rectangles $T$ having sides parallel to the axes. These families will be the rectangular analogues of the triangular families defined in the two preceding
sections.

We first consider the approximation of $\mathrm{H}^{1}$ functions. For any two nonnegative integers $k$ and $\ell$, denote by $P_{k, l}$ the space of polynomials in two variables, of degree at most $k$ in the first variable and of degree at most $\ell$ in the second variable, and by $\mathrm{P}_{k, \ell}(\mathrm{T})$ the space of restrictions to the rectangle $\mathrm{T}$ of the polynomials in $P_{k, l}$. To a rectangle $T$ and a positive integer $k$ is associated the set $\sum_{\mathrm{T}}^{(k)}$ of points of the grid on $\mathrm{T}$ obtained by subdividing each edge of $\mathrm{T}$ into $\mathrm{k}$ equal parts. The Lagrangian interpolant $L_{T}^{(k)} v$ on $T$ of order $k$ of a function $v \in C^{0}(T)$ is the unique function in $P_{k, k}(T)$ which coincides with $v$ at each point of $\sum_{T}^{(k)}$. For $T_{h}$ a triangulation of $\bar{\Omega}$ by rectangles, we define the interpolation operator $\mathcal{L}_{h}^{(k)}$ from $H^{1}(\Omega) \cap H^{2}\left(T_{h}\right)$ onto $L_{h}^{(k)}$, where $L_{h}^{(k)}$ is defined by

$$
\mathrm{L}_{\mathrm{h}}^{(\mathrm{k})}=\left\{\mathrm{v} \in \mathrm{H}^{1}(\Omega) ; \forall \mathrm{T} \in T_{\mathrm{h}}, \mathrm{v}_{\mathrm{T}} \in \mathrm{P}_{\mathrm{k}, \mathrm{k}}(\mathrm{T})\right\}
$$

by requiring that $\mathcal{L}_{\mathrm{h}}^{(\mathrm{k})} \mathrm{v}$ agree on $\mathrm{T}$ with $\mathcal{L}_{\mathrm{T}}^{(\mathrm{k})}\left(\mathrm{v}_{\mathrm{T}}\right)$ for each $\mathrm{T} \in T_{\mathrm{h}}$. The error estimate for Lagrangian interpolation given by Theorem 5.2 remain valid when the family of triangulations is a regular family of triangulations by rectangles; i.e. if $\left\{T_{h}\right.$; $h \in H\}$ is a family of triangulations $T_{h}$ of $\bar{\Omega}$ by rectangles $T$ of diameter $h_{T} \leq h$ which is regular in the sense that the ratio of the width to the length of each rectangle $T, T \in T_{h}, h \in H$, is bounded below by a positive constant independent of $h$, then there exists a constant $C$ such that 


$$
\left|v-z_{h}^{(k)} v\right|_{1, \Omega}+h^{-1}\left\|v-z_{h}^{(k)} v\right\|_{0, \Omega} \leq \mathrm{Ch}^{\mathrm{m}}|\mathrm{v}|_{\mathrm{m}+1, \Omega}
$$

$$
\text { for all } v \in \mathrm{H}^{\mathrm{m+1}}(\Omega) \text { with } 1 \leq \mathrm{m} \leq \mathrm{k} \text {. }
$$

The demonstration for this case may be found in RAVIART \& THOMAS (1983,pp. 79-103), for example.

For the approximation of vector functions on a rectangle $T$ we define the space $D_{k}(T)$ to be the product space $P_{k, k-1}(T) \times P_{k-1, k}(T)$. The equilibrium interpolant $E_{\mathrm{T}}^{(\mathrm{k})} \mathrm{g}$ of order $\mathrm{k}, \mathrm{k} \geq 1$, on $\mathrm{T}$ of the vector function $\mathrm{q} \in H$ (div; $\mathrm{T}$ ) is the unique function in $D_{k}(T)$ such that

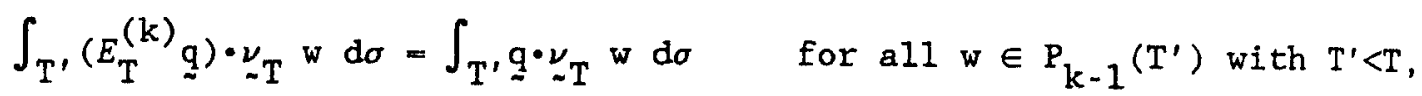

and

$$
\int_{T}\left(E_{h}^{(k)} q\right) \cdot \underline{\sim} d x=\int_{T} \underset{\sim}{q} \underline{\sim}_{r} d x \quad \text { for all } \underset{\sim}{r} \in P_{k-2, k-1}(T) \times P_{k-1, k-2}(T)
$$

For $T_{h}$ a triangulation of $\bar{n}$ by rectangles we define the interpolation operator $E_{h}^{(k)}$ from $H(\operatorname{div} ; \Omega) \cap H\left(\operatorname{div} ; T_{h}\right)$ onto $E_{h}^{(k)}$, where $E_{h}^{(k)}$ is defined by

$$
\mathrm{E}_{\mathrm{h}}^{(\mathrm{k})}=\left\{\underline{q} \in \mathrm{H}(\operatorname{div} ; \Omega) ; \forall \mathrm{T} \in T_{\mathrm{h}}, \mathrm{q}_{\mathcal{I}_{\mathrm{T}}} \in \mathrm{D}_{\mathrm{k}}(\mathrm{T})\right\} \text {, }
$$

by requiring that $E_{h}^{(k)} q$ agree on $T$ with $E_{T}^{(k)}\left(q_{T}\right)$ for each $T \in T_{h}$. Using Green's formula one can show that on each rectangle $T$

$$
\operatorname{div}\left(E_{\mathrm{T}}^{(\mathrm{k})} \mathrm{q}\right)=\Pi_{\mathrm{T}}^{(\mathrm{k}-1)}(\operatorname{div} \mathrm{q})
$$

for all $\mathrm{q} \in H(\operatorname{div} ; \mathrm{T})$,

where $\Pi_{T}^{(k-1)}$ is the orthogonal $L^{2}$ projection of $L^{2}(T)$ onto $P_{k-1, k-1}(T)$. The error estimate given by Theorem 6 remains valid in the rectangular case : if $\left\{T_{h} ; h \in H\right\}$ is a regular family of triangulations of $\bar{\Omega}$ by rectangles then there is a constant C such that

$$
\left\|\underline{q}-E_{\mathrm{h}}^{(\mathrm{k})} \mathrm{q}\right\|_{0, \Omega} \leq\left.\left.\mathrm{Ch} \mathrm{h}^{\ell}\right|_{\mathfrak{q}}\right|_{\ell, \Omega}
$$

for all $\underset{q}{\mathrm{q}} \in\left(\mathrm{u}^{\ell}(a)\right)^{2}$ with $1 \leq \ell \leq k$,

and 
$(7.8)$

$$
\left\|\operatorname{div}\left(q-E_{h}^{(k)} q\right)\right\|_{0, \Omega} \leq \mathrm{Ch}^{2}|\operatorname{div} \mathrm{q}|_{\ell, \Omega}
$$

for all $\underline{q} \in\left(\mathrm{H}^{1}(\Omega)\right)^{2}$ with div $\underline{q} \in \mathrm{H}^{\ell}(\Omega), 0 \leq \ell \leq k$.

For the demonstration in the case $n=2$ treated here, see THOMAS (1977, Chapter III) ; for the generalization to the case $n=3$, see NEDELEC (1980).

The analogue for rectangular elements of the second family described in Section 6 is obtained by choosing for $k \geq 2$ for interpolation space on the rectangle $\mathrm{T}$ the space

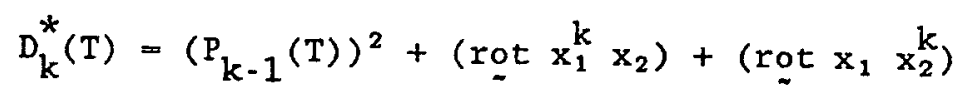

and for interpolation operator $E_{\mathrm{T}}^{*(\mathrm{k})}$ on $H($ div; $\mathrm{T})$ the mapping associating to $\mathrm{q} \in$ $H($ div; $T)$ the unique function in $D_{k}^{*}(T)$ such that

$$
\int_{T^{\prime}}\left(E_{T}^{*(k)} q\right) \cdot \nu_{T} w d \sigma=\int_{T^{\prime}} q_{*} \cdot \nu_{-T} w d o
$$

for all $w \in P_{k-1}\left(T^{\prime}\right)$ with $T^{\prime}<T$,

and

$$
\int_{T}\left(E_{T}^{*(k)} q\right) \cdot \underline{r} d x=\int_{T} \underline{q} \cdot \underline{r} d x
$$$$
\text { for all } \underset{\sim}{r} \in\left(P_{k-2}(T)\right)^{2} \text {. }
$$

Then to a triangulation $T_{h}$ of $\bar{\Omega}$ by rectangles we associate the interpolation space $E_{h}^{*(k)}$ and interpolation operator $E_{h}^{*(k)}$ from $H(d i v ; \Omega) \cap H\left(d i v ; T_{h}\right)$ onto $E_{h}^{*(k)}$ defined by analogy with the preceding example. The analogue of (7.6) holds in this case as do the error bounds (6.39) and (6.40). For the details of the construction and the demonstration of the error bounds see BREZZI, DOUGLAS, \& MARINI (1985, paragraph 5). The extension to the 3 dimensional case is given in BREZZI, DOUGLAS, DURAN, \& FORTIN (1987, paragraph 3).

$\frac{\text { Remark 7.1 }}{*}$. In the rectangular case the difference in size of the spaces $D_{k}(T)$ and $D_{k}^{*}(T)$ is more striking than in the triangular case. For $T$ a rectangle,

$$
\left\{\begin{array}{l}
\operatorname{dim} D_{k}(T)=2 k^{2}+2 k \\
\operatorname{dim} D_{k}^{*}(T)=k^{2}+k+2
\end{array}\right.
$$

and indeed, if NE is the number of elements in $T_{h}$, 


$$
\operatorname{dim} E_{h}^{(k)}-\operatorname{dim} E_{h}^{*(k)}=\left(k^{2}+k-2\right) N E=\left\{\begin{array}{l}
4 N E \text { if } k=2, \\
10 N E \text { if } k=3
\end{array}\right.
$$

However, at the level of construction of the spaces $E_{h}^{(k)}$ and $E_{h}^{*(k)}$, we note that the degrees of freedom which assure that the continuity condition (6.17) holds, i.e. those which assure the connections between the finite elements and thus contribute to the size of the algebraic system to be solved, are exactly the same for the two methods.

The situation is not the same in 3 dimensions. If $D_{k}(T)$ and $D_{k}^{*}(T)$ denote the local approximation spaces on a rectangular solid $\mathrm{T}$ as defined in NEDELEC (1980) and in BREZZI, DOUGLAS, DURAN \& FORTIN (1987) respectively, we have

$$
\left\{\begin{array}{l}
\operatorname{dim} D_{k}(T)=3 k^{2}(k+1) \\
\operatorname{dim} D_{k}^{*}(T)=3 / 2 k\left(k^{2}+3 k+8\right)
\end{array}\right.
$$

Given a triangulation $T_{h}$ of $\bar{\Omega}$ by rectangular solids, the degrees of freedom needed to guarantee the required continuity in the corresponding finite dimensional subspaces of $H\left(\operatorname{div}_{i} \Omega\right), \mathrm{E}_{h}^{(k)}$ and $E_{h}^{*(k)}$, are the moments of the normal component with respect to

$$
\begin{cases}P_{k-1, k-1}\left(T^{\prime}\right) & \text { (i.e. } k^{2} \text { moments) for elements of } E_{h}^{(k)}, \\ P_{k-1}\left(T^{\prime}\right) & \text { (i.e. } k(k+1) \text { moments) for elements of } E_{h}^{*(k)} .\end{cases}
$$

Here the gain in calculation time with interpolation by $E_{h}^{*(k)}$, the operator associated with $E_{h}^{*(k)}$, instead of $E_{h}^{(k)}$, the operator associated with $E_{h}^{(k)}$, is clear. If $N F$ is the number of 2-dimensional faces $T^{\prime}$ of elements $T$ of $T_{h}$ with $T^{\prime}$ $c \Omega$, then the difference in calculation time can be estimated in terms of the number

$$
\left(3 k^{2}-3 k\right) N F=\left\{\begin{array}{rll}
6 N F, & \text { if } k=2 \\
18 N F, & \text { if } k=3
\end{array}\right.
$$


Nevertheless, concerning the precision of the interpolation in 3 dimensions as well as in 2 dimensions, we note as in the simplicial case (cf. Remark 6.5) that while the order of approximation is the same for $E_{h}^{(k)}$ and $E_{h}^{*(k)}$ in $\left(I^{2}(\Omega)\right)^{n}$, the accuracy in $H(\operatorname{div} ; \Omega)$ of $E_{h}^{*(k)}$ is of one order less than that of $E_{h}^{(k)}$.

Remark 7.2 - Another family of examples of H(div) approximation on rectangles in 2 dimensions and in 3 dimensions may be found in BREZZI, DOUGLAS, FORTIN, \& MARINI (1987). For rectangular elements in 3 dimensions as well as for prisms, other families are given in NEDELEC (1986).

We cannot develop here a theory of approximation as general as that constructed above for finite elements of such an elementary form as a triangle or a rectangle for the case of finite elements of other geometric forms. However, we shall describe an example where the triangulation $T_{h}$ of $\bar{\Omega}$ consists of convex quadrilaterals. Thus for $T \in T_{h}$ there exists a bijection $F \in\left(P_{1,1}\right)^{2}$ such that $T=$ $F(\hat{T})$, with $\hat{T}$ the reference square having vertices $(0,0),(1,0),(1,1)$ and $(0,1)$. For each point $\hat{x} \in \dot{T}$, the linear tangent mapping $D F(\hat{x})$ and its $J$ acobien $J(\hat{x})=$ $\operatorname{det}(\operatorname{DF}(\hat{x}))$ are affine functions of the coordinates $\left(\hat{x}_{1}, \hat{x}_{2}\right)$.

For a scalar function $\mathrm{v} \in \mathrm{H}^{2}(\mathrm{~T})$ we may define a function $\mathscr{L}_{\mathrm{T}}^{(1)} \mathrm{v}$ in the space

$$
\left\{w \in H^{1}(T) ; w \circ F \in P_{1,1}(\hat{T})\right\}
$$

by interpolating the values of $\mathrm{v}$ at the four vertices of the quadrilateral $\mathrm{T}$.

Similarly for a vector function $\mathrm{q} \in H$ (div; $T$ ) we may interpolate, on each of the four edges, the average value of the normal trace to define a function $E_{\mathrm{T}}^{(1)} \mathrm{q}$ in the space

$$
\left\{\underline{\sim} \in H(\operatorname{div} ; T) ;|J| \mathrm{DF}^{-1}(\underline{r} \circ \mathrm{F}) \in \mathrm{P}_{2,1}(\hat{\mathrm{T}}) \times \mathrm{P}_{1,2}(\hat{\mathrm{T}})\right\}
$$

Even though the spaces defined in (7.17) and (7.18) are spaces of polynomial functions only if $T$ is a parallelogram, we note that in any case the trace of $\mathcal{L}_{T}^{(1)}$ on each edge $T^{\prime}$ of $T$ is an affine function and the normal trace of $E_{T}^{(1)} \mathrm{q}$ on $T^{\prime}$ is constant. Furthermore, $|\mathrm{J}| \operatorname{div}\left(E_{\mathrm{T}}^{(1)} \mathrm{q}\right)$ is constant on $\mathrm{T}$ and we have

$$
|\mathrm{J}| \operatorname{div}\left(E_{\mathrm{T}}^{(1)} \mathrm{q}\right)=\frac{1}{\operatorname{mes} \hat{\mathrm{T}}} \int_{\mathrm{T}} \operatorname{div} \mathrm{q} \mathrm{dx} .
$$

Proceeding as in each of the preceding examples, by patching together the maps $\mathcal{L}_{\mathrm{T}}^{(1)}, \mathrm{T} \in T_{\mathrm{h}}$, we define an operator $\mathcal{L}_{\mathrm{h}}^{(1)}$ on $\mathrm{H}^{1}(\Omega) \cap \mathrm{H}^{2}\left(T_{\mathrm{h}}\right)$ having as image 


\section{CHAPTER III}

\section{ABSTRACT THEORY OF APPROXIMATION OF SOLUTIONS OF PROBLEMS}

\section{WITH LAGRANGIAN MULTIPLIERS}

\section{8 - GENERAL REMARRS}

In this chapter we develop an abstract theory for the approximation of solutions of problems with Lagrangian multipliers. The main purpose for the construction of such a theory is to unify the analysis of the mixed and hybrid methods presented in Chapter $I$, and hence to provide a framework for generalizations and extensions of the analysis to that of similar problems.

Even though these methods were conceived of in the context of approximation of problems of minimization of a quadratic functional with affine constraints, the properties of constrained optimization are not at all fundamental to the analysis of the problems studied here. On the contrary, more than one familiar with conforming approximations of minimization problems seems to have been misguided by this approach. In the exposition of the abstract theory given in this chapter, we will make no restrictions which allow the class of problems studied here to be interpreted as being minimization problems on an affine manifold. The expression "with Lagrangian multiplier" will be used in the same formal sense as is the expression "variational problem".

Let us now give the general framework for this study. Let $W$ and $M$ be Hilbert spaces with inner products $((\cdot, \cdot))_{W}$ and $((\cdot, \cdot))_{M}$ respectively. The norms associated with these inner products are denoted $\|\cdot\|_{W}$ and $\|\cdot\|_{M}$. Further, let $a(\cdot, \cdot)$ and $b(\cdot, \cdot)$ be bilinear forms defined on $W \times W$ and on $W \times M$ respectively. Given the linear forms $f(\cdot)$ defined on $W$ and $g(\cdot)$ defined on $M$, we consider the following problem : find a pair $(\phi, \lambda)$ satisfying

$$
\begin{cases}(\phi, \lambda) \in \mathrm{W} \times \mathrm{M}, & \\ \mathrm{a}(\phi, \psi)+\mathrm{b}(\psi, \lambda)=\mathrm{f}(\psi) & \text { for all } \psi \in \mathrm{W} \\ \mathrm{b}(\phi, \mu) & \text { for all } \mu \in \mathrm{M}\end{cases}
$$


We introduce the product space $X=W \times M$ which is a Hilbert space with norm

$$
\|v\|_{X}=\left(\|\psi\|_{W}^{2}+\|\mu\|_{M}^{2}\right)^{\frac{1}{2}} \quad \text { for all } v=(\psi, \mu) \in X
$$

Clearly one may also formulate $(8,1)$ in the following manner : find $u=(\phi, \lambda)$ satisfying

$$
\left\{\begin{array}{l}
u \in X, \\
k(u, v)=\ell(v) \quad \text { for all } v \in X,
\end{array}\right.
$$

where $k(\cdot, \cdot)$ is a bilinear form on $\mathrm{X} \times \mathrm{X}$ given by

$$
\begin{array}{ll}
\mathrm{k}(\mathrm{u}, \mathrm{v})=\mathrm{a}(\phi, \psi)+\mathrm{b}(\psi, \lambda)+\mathrm{b}(\dot{\phi}, \mu) \quad \text { for all } \mathrm{u}=(\phi, \lambda) \in \mathrm{X} \text { and } \\
\text { for all } \mathbf{v}=(\psi, \mu) \in \mathrm{X}
\end{array}
$$

and where $\ell(\cdot)$ is a linear form defined on $X$ by

$$
\ell(v)=f(\psi)+g(\mu) \quad \text { for all } v=(\psi, \mu) \in X
$$

All questions of continuity aside, it is evident that the analysis of the variational problem (8.2) can not be carried out with the aid of the Lax-Milgram theorem since the bilinear form $k(\cdot, \cdot)$ is not $X$-elliptic : for each $v=(0, \mu) \in X$, we have $k(v, v)=0$. One may show, nevertheless, the following result :

THEOREM 8.1. Suppose that the bilinear form $\mathrm{k}(\cdot, \cdot)$ is continuous on $\mathrm{X} \times \mathrm{X}$ and that it satisfies

i)

$$
\inf _{\left\{u \in X ;\|u\|_{X}=1\right\}} \quad \begin{aligned}
& \text { sup } \\
& \left\{v \in X ;\|v\|_{X}=1\right\}
\end{aligned}
$$

and

ii).

$$
\sup _{\left\{u \in X ;\|u\|_{X}=1\right\}} k(u, v)>0
$$

$k(u, v)>0$,

for all $\mathrm{v} \in \mathrm{X}$ with $\mathrm{v} \neq 0$.

Then for each continuous linear form $\ell(\cdot)$ on $\mathbf{X}$, there exists a unique solution of problem (8.2). 
This theorem is a special case of the theorem due to BABUSKA [1971] given in the following section.

Remark 8.1. Hypothesis ii) is superfluous when the bilinear form $k(\cdot, \cdot)$ is symmetric because, in this case, i) implies ii).

\section{9 - ANALYSIS AND APPROXIMATION OF THE SOLUTION OF A VARIATIONAL}

\section{PROBLEM (THEORY OF BABUSKA)}

We consider the two Hilbert spaces $X$ and $Y$ equipped with the norms $\|\cdot\|_{X}$ and $\|\cdot\|_{Y}$, and let $k(\cdot, \bullet)$ be a bilinear form on $X \times Y$. We have

THEOREM 9.1. Suppose that $\mathrm{k}(\cdot, \cdot)$ is a continuous, bilinear form on $\mathrm{X} \times \mathrm{Y}$ which satisfies

$$
\text { i) } \begin{array}{lll}
\inf & \text { sup } & k(u, v)>0,
\end{array}
$$

and

ii)

$$
\begin{array}{lll}
\sup & k(u, v)>0 & \text { for all } v \in Y \text { with } v \neq 0 .
\end{array}
$$

Then for each continuous linear form $\ell \cdot$ ) on $\mathrm{Y}$, the problem: find $\mathrm{u}$ satisfying

(9.1) $\left\{\begin{array}{l}u \in X, \\ k(u, v)=\ell(v)\end{array}\right.$

$$
\text { for all } \mathrm{v} \in \mathrm{Y} \text {, }
$$

has a unique solution.

Proof. Define the continuous linear operator $K$ from $X$ to $Y$ by

$$
((K u, v))_{Y}=k(u, v) \quad \text { for all } u \in X \text { and for all } v \in Y \text {. }
$$


Hypothesis i) implies that the operator $K$ is injective and that its image is closed in $Y$; hypothesis ii) expresses the fact that the image of $K$ is dense in $Y$.

Remark 9.1. Conditions $i$ ) and ii) together give a necessary and sufficient condition for problem (9.1) to have a unique solution given a continuous, linear form $\ell(\cdot)$. In the case $X=Y$, the ellipticity condition,

$$
\begin{aligned}
& \inf \\
& \left\{v \in X ;\|v\|_{X}=1\right\}
\end{aligned}
$$

obviously implies conditions $i$ ) and $i$ ); thus it is a sufficient condition for problem (9.1) to have a unique solution. In this sense Theorem 9.1 generalizes the Lax-Milgram theorem.

In the next section we shall come back to and treat in detail the "particular case" where $X=W \times M$ and $k(\cdot, \cdot)$ is of the form (9.3), i.e. the case that motivates our study. First, however, we give a general result concerning the approximation of the solution of problem (9.1). Given finite dimensional subspaces $X_{h}$ and $Y_{h}$ of $X$ and $Y$ respectively, we seek the "approximate solution" $u_{h} \in X_{h}$, an approximation of the solution $u$ of problem (9.1), defined by

$$
\left\{\begin{array}{l}
u_{h} \in x_{h}, \\
k\left(u_{h}, v_{h}\right)=\ell\left(v_{h}\right)
\end{array} \quad \text { for all } v_{h} \in Y_{h} .\right.
$$

Applying Theorem 9.1 in the finite dimensional case, we obtain :

THEOREM 9.2. Suppose that $\mathrm{k}(\cdot, \cdot)$ is a bilinear form on $\mathrm{X}_{\mathrm{h}} \times \mathrm{Y}_{\mathrm{h}}$ which satisfies

and

$$
\text { i) } \begin{array}{ll}
\inf & \sup \\
\left(u_{h} \in X_{h} ; \| u_{h}, v_{h}\right)>0, &
\end{array}
$$

ii) $\sup _{\left\{\mathrm{u}_{\mathrm{h}} \in \mathrm{X}_{\mathrm{h}} ;\left\|\mathrm{u}_{\mathrm{h}}\right\|_{\mathrm{X}}=1\right\}} \mathrm{k}\left(\mathrm{u}_{\mathrm{h}}, \mathrm{v}_{\mathrm{h}}\right)>0 \quad$ for all $\mathrm{v}_{\mathrm{h}} \in \mathrm{Y}$ with $\mathrm{v}_{\mathrm{h}} \neq 0$.

Then for each linear form $\ell(\cdot)$ on $\mathrm{Y}_{\mathrm{h}}$, problem (9.2) has a unique solution. 
Remark 9.2. The space $\mathrm{X}_{\mathrm{h}}$ being finite dimensional, condition i) is equivalent to

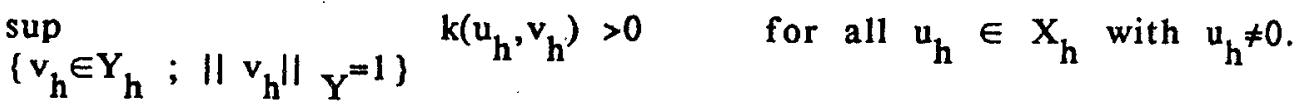

Futhermore, when the hypotheses of Theorem 9.2. are realized, one has necessarily that

$$
\operatorname{dim} Y_{h}=\operatorname{dim} X_{h}
$$

Conversely, when we know, a priori, that (9.3) is satisfied (for example when $X_{h}=Y_{h}$ ), it suffices to check that either one or the other of conditions i) and $i$ ) of Theorem 9.2 holds.

Remark 9.3. It is important to note that it is not sufficient that hypotheses $i$ ) and ii) of Theorem 9.1 be satisfied in order that the corresponding conditions of Theorem 9.2 be also. Herein lies the charm of the analysis !

Remark 9.4. Even when $\mathrm{X}=\mathrm{Y}$ and the bilinear form $\mathrm{k}(\cdot, \cdot)$ is $\mathrm{X}$-elliptic, it can be useful to consider examples for which (9.2) holds with $X_{h} \neq Y_{h}$. Such is, for instance, the case in a Petrov-Galerkin type approach to a diffusion-convection problem, cf, for example MIZUKAMI \& HUGHES [1985].

We conclude this section with an estimation of the error committed in approximating the solution $u$ of problem (9.1) by the solution $u_{h}$ of problem (9.2) :

THEOREM 9.3 Suppose that $\mathrm{K}(\cdot, \cdot)$ is a continuous, bilinear form on $\mathrm{X} \times \mathrm{Y}$ and let $\mathrm{K}$ be a number such that

$$
\sup _{\left\{v \in Y ;\|v\|_{Y}=1\right\}} k(u, v) \leq K\|u\|_{X} \quad \text { for áll } u \in X
$$


Suppose that the choice of subspaces $\mathrm{X}_{\mathrm{h}}$ and $\mathrm{Y}_{\mathrm{h}}$ is such that

$$
\operatorname{dim} Y_{h}=\operatorname{dim} X_{h}
$$

and that there exists a number $\kappa>0$ such that

$$
\sup _{\left\{v_{h} \in Y_{h} ;\left\|v_{h}\right\|_{Y}=1\right\}} k\left(u_{h}, v_{h}\right) \geq \kappa\left\|u_{h}\right\|_{X} \quad \text { for all } u_{h} \in X_{h} \text {. }
$$

Then for each linear form $\left.\ell^{\cdot}\right)$ on $\mathrm{Y}$, if $\mathrm{u}$ satisfies (9.1) and $\mathrm{u}_{\mathrm{h}}$ is the solution to (9.2), we have the error bound

$$
\left\|u-u_{h}\right\|\left\|_{X} \leq\left(1+\frac{K}{\kappa}\right) \inf _{w_{h} \in X_{h}}\right\| u-w_{h} \|_{X} .
$$

This result is due to BABUSKA [1971] ; cf. also BABUSKA \& AZIZ [1972, Theorem 6.2.1, p. 1861); a simple demonstration in the case $X_{h}=Y_{h}$ can be found in RABIER \& THOMAS [1985, Exercise 3.13], for example. For a given problem of the form (9.1), the constants $K$ and $\kappa$ which appear in (9.4) and (9.6), respectively, obviously depend on the choice of norm on $X$ and on $Y$ and on the choice of the subspaces $X_{h}$ and $Y_{h}$ - nothing prevents one from making the choice of the norms as a function of the choice of the finite dimensional subspaces. We will see some such examples later on.

For the moment we shall settle for a simple criterion for convergence when the index $h$, indicating the choice of finite dimensional subspace $x_{h}$ as well as a real parameter, tends toward 0 . The set of parameters will be denoted $H$.

THEOREM 9.4. Suppose that the norms \|\|$_{\mathrm{X}}$ and \|\|$_{\mathrm{Y}}$ on the spaces $\mathrm{X}$ and $\mathrm{Y}$, respectively, are chosen independently of the parameter $\mathrm{h} \in H$ and that condition (9.4) is satisfied. Suppose that for each $\mathrm{h} \in H$, we have been able to choose the finite dimensional subpaces $\mathrm{X}_{\mathrm{h}}$ and $\mathrm{Y}_{\mathrm{h}}$ to be of the same dimension and such that condition (9.6) is realized with a constant $\kappa>0$ independent of h. Suppose further that there exist a subspace $X$ of $\mathrm{X}$ dense in $\mathrm{X}$ and a mapping $\mathrm{r}_{\mathrm{h}}$ of $X$ into $\mathrm{X}_{\mathrm{h}}$ such that

$$
\lim _{h \rightarrow 0}\left\|w-r_{h}(w)\right\|_{X}=0 \quad \text { for all } w \in X \text {. }
$$

Then, the variational approximation method converges in the sense that we have 


$$
\lim _{h \rightarrow 0}\left\|u-u_{h}\right\|_{X}=0
$$

where $\mathrm{u}$ is the solution of (9.1) and $\mathrm{u}_{\mathrm{h}}$ the solution of (9.2).

The demonstration of this theorem is identical to that showing the convergence of the approximate solutions of an elliptic variational problem, cf. for example RAVIART \& THOMAS [1983, Theorem 3.1-3].

\section{0 - ANALYSIS AND APPROXIMATION OF THE SOLUTION OF A VARIATIONAL}

\section{PROBLEM (THEORY OF BREZZI)}

Equipped with the general results of the preceding section, we proceed with the analysis of a problem of the form (8.1) which we shall call a problem with Lagrangian multipliers : find a pair $(\phi, \lambda)$ satisfing

$$
\begin{cases}(\phi, \lambda) \in \mathrm{W} \times \mathrm{M}, & \\ \mathrm{a}(\phi, \psi)+\mathrm{b}(\psi, \lambda)=\mathrm{f}(\psi) & \text { for all } \psi \in \mathrm{W} \\ \mathrm{b}(\phi, \mu) & \text { for all } \mu \in \mathrm{M}\end{cases}
$$

In particular if a pair $(\phi, \lambda)$ satisfies $(10.1)$ with $g(\cdot)=0$, then the first argument $\phi$, is a solution of the variational problem : find $\phi$ such that

$$
\left\{\begin{array}{l}
\phi \in \mathrm{V}, \\
\mathrm{a}(\phi, \psi)=\mathrm{f}(\psi)
\end{array}\right.
$$$$
\text { for all } \psi \in \mathrm{V} \text {, }
$$

where $V$ is the subspace of $W$ defined by

$$
\mathrm{V}=\{\psi \in \mathrm{W} ; \forall \mu \in \mathrm{M}, \mathrm{b}(\psi, \mu)=0\}
$$

The following theorem is due to BREZZI [1974a, 1974b]:

THEOREM 10.1. Suppose that the bilinear form $\mathrm{a}(\cdot, \cdot)$ is continuous on $\mathrm{W} \times \mathrm{W}$ and $\mathrm{V}$-elliptic, i.e.

i)

$$
\inf _{\left\{\psi \in V ;\|\psi\|_{W}=1\right\}}^{a(\psi, \psi)>0}
$$


Suppose that the bilinear form $\mathrm{b}(\cdot, \cdot)$ is continuous on $\mathrm{W} \times \mathrm{M}$ and that it satisfies

ii)

$$
\begin{aligned}
& \text { inf sup } \quad b(\psi, \mu)>0 \text {. } \\
& \left\{\mu \in M ;\|\mu\|_{M}=1\right\}\left\{\psi \in W ;\|\psi\|_{W}=1\right\}
\end{aligned}
$$

Then for each pair of continuous linear forms $f(\cdot)$ on $\mathrm{W}$ and $g(\cdot)$ on $\mathrm{M}$, problem (10.1) has a unique solution.

Proof. The original demonstration of this theorem is given in BREZZI [1974b]; a complete demonstration may also be found in GIRAULT \& RAVIART [1979, Theorem 4.1] for example. We shall indicate here a variant of the demonstration of this fundamental result. Let $V^{\downarrow}$ denote the subspace of $W$ orthogonal to $V$; denote by $\psi^{0}$ and $\psi^{\perp}$ the orthogonal projections of $\psi \in W$ in $V$ and $V^{\perp}$, respectively. With this notation, problem (10.1) may be written: find $\phi^{\perp}, \phi^{0}$, and $\lambda$ such that

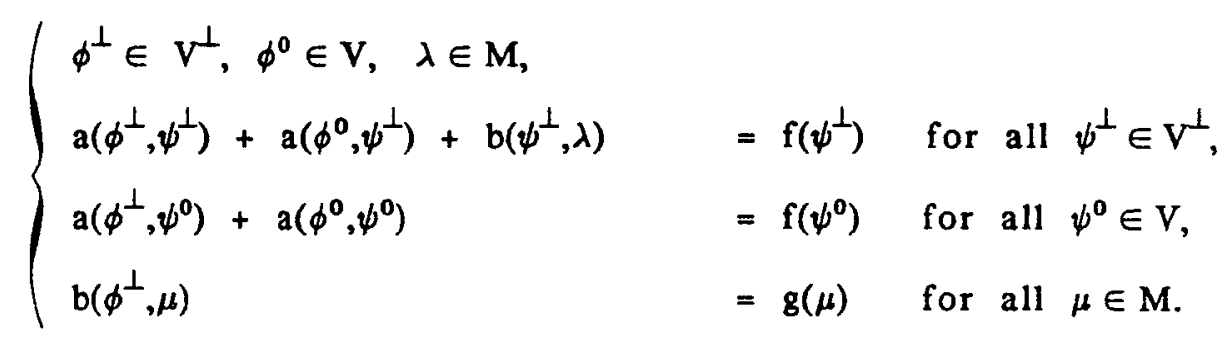

Note that we have the equality

$$
\begin{array}{llll}
\sup & \mathrm{b}(\psi, \mu) & \sup & \mathrm{b}(\psi, \mu) \\
\left\{\psi \in \mathrm{V}^{\perp} ;\|\psi\|_{\mathrm{W}}=1\right\}
\end{array} \quad \text { for all } \mu \in \mathrm{M} \text {, }
$$

and that we have the characterization of $V$ as a subset of $W$ :

$$
\psi \in \mathrm{V} \quad \text { if and only if } \sup _{\left\{\mu \in \mathrm{M} ;\|\mu\|_{\mathrm{M}}=1\right\}} \quad \mathrm{b}(\psi, \mu)=0
$$

Using hypothesis ii) of Theorem 10.1 one may then establish with the aid of Theorem 9.1 that the linear operator $B^{*}$ from $M$ into $V^{\perp}$ defined by

$$
\left(\left(\hat{\psi}, D^{*}{ }_{\mu j}\right)\right)_{\mathrm{W}}=b(\hat{\psi}, \mu) \quad \text { for ani } \dot{\psi} \in \mathrm{W}, \mu \in \mathrm{Mi}
$$


is an isomorphism of $M$ onto $V^{\perp}$. Thus the linear operator $B$ from $V^{\perp}$ into $M$ defined by

$$
((B \psi, \mu))_{M}=\left(\left(\psi, B^{*} \mu\right)\right)_{W} \quad \text { for all } \psi \in W, \mu \in \mathrm{M}
$$

is an isomorphism of $V^{\perp}$ onto $M$.

Finally, using hypothesis i) of Theorem 10.1 one may show with the aid of the Lax Milgram theorem that the linear operator $A$ from $V$ into $V$ defined by

$$
((A \phi, \psi))_{\mathrm{W}}=\mathrm{a}(\phi, \psi) \quad \text { for all } \phi \in \mathrm{V}, \psi \in \mathrm{W},
$$

is an automorphism. Thus the linear system (10.4), to which is associated a matrix of operators of the form

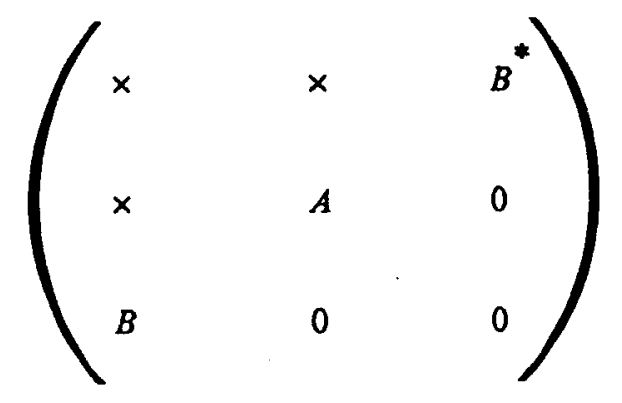

has a unique solution.

Remark 10.1. Condition ii) of Theorem 10.1 is often called the inf-sup condition; some authors refer to it as the Brezzi condition, others as the Brezzi-Babuska condition. The current trend seems to be to use the expression LBB condition, where LBB stands for LADYZHENSKAYA [1949] - BABUSKA [1971] - BREZZI [1974].

Remark 10.2. A necessary and sufficient condition for problem (10.1) to have a unique solution $(\phi, \lambda) \in W \times M$ given the linear forms $f(\cdot)$ on $W$ and $g(\cdot)$ on $M$ is that the inf-sup condition be satisfied and that the operator $A \in \mathscr{L}(\mathrm{V} ; \mathrm{V})$ associated with the bilinear form $a(\cdot, \cdot)$ be an isomorphism. This result, through presented differently, can be found in GIRAULT \& RAVIART [1985, Theorem 4.1]. 
With the goal of approximating the solution of (10.1), we take now finite dimensional subspaces of $W$ and of $M$,

$$
\mathbf{w}_{h} \subset \mathbf{W}, \quad \mathbf{M}_{\mathbf{h}} \subset \mathbf{M}
$$

and define the discrete problem : find $\left(\phi_{h}, \lambda_{h}\right)$ satisfying

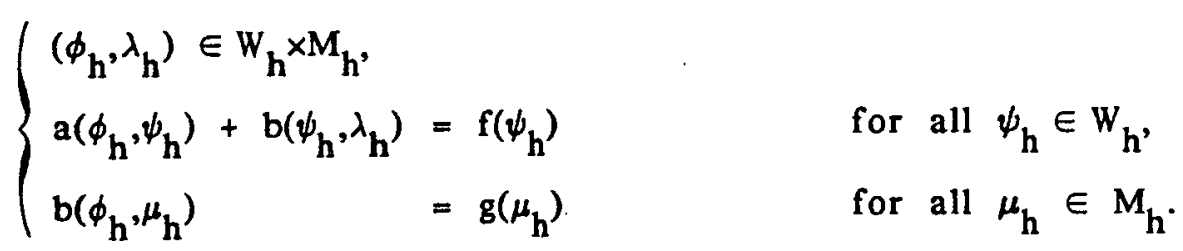

As in the continuous case, we introduce the subspace $V_{h}$ of $W_{h}$ given by

$$
\mathrm{V}_{\mathrm{h}}=\left\{\psi_{\mathrm{h}} \in \mathrm{W}_{\mathrm{h}} ; \forall \mu_{\mathrm{h}} \in \mathrm{M}_{\mathrm{h}}, \mathrm{b}\left(\psi_{\mathrm{h}}, \mu_{\mathrm{h}}\right)=0\right\}
$$

One should be careful to note that in general $V_{h}$ is not a subspace of the space $\mathrm{V}$ defined in (10.3). Transposing to the finite dimensional case the results of Theorem 10.1, we obtain

\section{THEOREM 10.2. Suppose that}

i) the bilinear form $\mathrm{a}(\cdot, \cdot)$ is $\mathrm{V}_{\mathrm{h}}$-elliptic

and

ii) the bilinear form $b(\cdot, \cdot)$ satisfies

$$
\inf _{\left\{\mu_{h} \in M_{h} ;\left\|\mu_{h}\right\|_{M}=1\right\}} \sup _{\left\{\psi_{h} \in W_{h} ;\left\|\psi_{h}\right\|_{W}=1\right\}}^{b\left(\psi_{h}, \mu_{h}\right)>0}
$$

Then for each pair of linear forms $f(\cdot)$ on $\mathrm{W}_{\mathrm{h}}$ and $\mathrm{g}(\cdot)$ on $\mathrm{M}_{\mathrm{h}}$, problem (10.6) has a unique solution.

Remark 10.3. Condition ii) of Theorem 10.2 is called the discrete inf-sup condition or again the discrete LBB condition. It says that the choice of the subspace $W_{h}$ of $W$ and that of the subspace $M_{h}$ of $M$ cannot be made independently one 
from the other, that there is a compatibility relation between the two subspaces. If we introduce the bases $\left(\psi_{h}^{(j)}\right)_{j=1, \ldots, d i m W_{h}}$ and $\left(\mu_{h}^{(i)}\right)_{i=1, \ldots, d i m M_{h}}$ of the subspaces $W_{h}$ and $M_{h}$ respectively, the discrete inf-sup condition may be interpreted as expressing the fact that the matrix $\left[B_{h}\right]$ whose $i, j$ entry is $\left[B_{h}\right]_{i, j}=b\left(\psi_{h}^{(i)}, \mu_{h}^{(i)}\right)$ has rank equal to the dimension of $M_{h}$ :

$$
\operatorname{rg}\left[B_{h}\right]=\operatorname{dim} M_{h} \text {. }
$$

In this form, the condition has been called the rank condition (cf. BABUSKA, ODEN \& LEE, [1978]. Clearly a necessary condition for this condition to hold is that

$$
\operatorname{dim} M_{h} \leq \operatorname{dim} W_{h} .
$$

Remark 10.4. Obviously if the bilinear form $a(\cdot, \cdot)$ is V-elliptic and $\mathrm{V}_{\mathrm{h}} \subset \mathrm{V}$ (as will be the case for dual mixed methods), then $a(\cdot, \cdot)$ is $V_{h}$-elliptic, or better yet if $a(\cdot, \cdot)$ is W-elliptic, so one no longer needs to have $V_{h} \subset V$ (as will be the case for dual hybrid and primal mixed methods), then $a(\cdot, \cdot)$ is $V_{h}$-elliptic. In other cases it is necessary to verify directly the $V_{h}$-ellipticity of $a(\cdot, \cdot)$, something which can be rather technical (this will be the situation for primal hybrid methods).

To verify the discrete inf-sup condition one can use the following result (cf. FORTIN, [1977]), the proof of which is immediate :

THEOREM 10.3. Suppose there is a Hilbert space WCW, an operator $R_{\mathrm{h}}$ from $\mathrm{M}_{\mathrm{h}}$ into $W$ satisfying

$$
\frac{\mathrm{b}\left(R_{\mathrm{h}} \mu_{\mathrm{h}}, \mu_{\mathrm{h}}\right)}{\left\|R_{\mathrm{h}} \mu_{\mathrm{h}}\right\|_{W}} \geq \mathrm{C} \sup _{\left\{\psi \in \mathrm{W} ;\|\psi\|_{\mathrm{W}}=1\right\}} \mathrm{b}\left(\psi, \mu_{\mathrm{h}}\right) \quad \text { for all } \mu_{\mathrm{h}} \in \mathrm{M}_{\mathrm{h}} \text {, }
$$

for some $\mathrm{C} \geq 0$, and a continuous linear operator $\pi_{h}$ from $W$ into $\mathrm{W}_{\mathrm{h}}$ such that the following diagram commutes : 


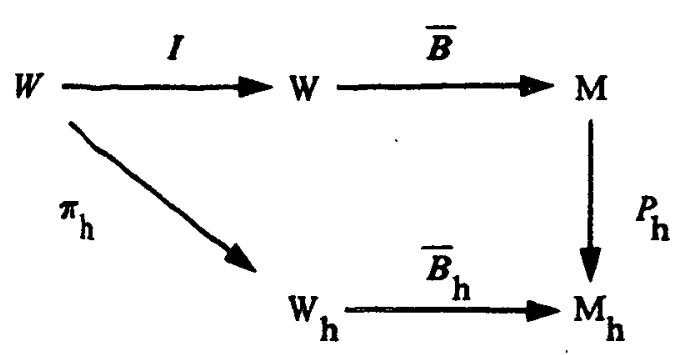

where $I$ is the inclusion of $W$ into $\mathrm{W}, \bar{B}$ and $\bar{B}_{\mathrm{h}}$ are the mappings from $\mathrm{W}$ to $\mathrm{M}$ and from $\mathrm{W}_{\mathrm{h}}$ to $\mathrm{M}_{\mathrm{h}}$, respectively, induced by $\mathrm{b}(\cdot, \cdot)^{(1)}$ and $P_{\mathrm{h}}$ is the orthogonal projection of $\mathrm{M}$ onto $\mathrm{M}_{\mathrm{h}}$. Then the inf-sup condition of Theorem 10.1 implies the discrete inf-sup condition of Theorem 10.2.

We give an estimate of the error made in approximating the solution of problem (10.1) by that of problem (10.6). This estimate was obtained by BREZZI $[1974 a, 1974 b]$.

THEOREM 10.4. Suppose that $\mathrm{a}(\cdot, \cdot)$ and $\mathrm{b}(\cdot, \cdot)$ are continuous bilinear forms on $\mathrm{W} \times \mathrm{W}$ and on $\mathrm{W} \times \mathrm{M}$ respectively. Let $\mathrm{A}$ and $\mathrm{B}$ be numbers such that

$$
\begin{array}{ll}
\mathrm{a}(\phi, \psi) \leq \mathrm{A} \quad\|\phi\|_{\mathrm{W}}\|\psi\|_{\mathrm{W}} & \text { for all } \phi \in \mathrm{W} \text { and } \psi \in \mathrm{W}, \\
\mathrm{b}(\psi, \mu) \leq \mathrm{B}\|\psi\|_{\mathrm{W}}\|\mu\|_{\mathrm{M}} & \text { for all } \psi \in \mathrm{W} \text { and } \mu \in \mathrm{M} .
\end{array}
$$

Suppose further that the subspaces $\mathrm{W}_{\mathrm{h}}$ and $\mathrm{M}_{\mathrm{h}}$ have been chosen such that there exist positive numbers $\alpha$ and $\beta$ for which we have

$$
a\left(v_{h}, v_{h}\right) \geq \alpha\left\|v_{h}\right\|_{W}^{12} \quad \text { for all } v_{h} \in v_{h}
$$

$$
\begin{array}{lll}
\inf & \sup & b\left(\psi_{h}, \mu_{h}\right) \geq \beta \\
\left\{\mu_{h} \in M_{h} ;\left\|\mu_{h}\right\|_{M^{\prime}}=1\right\} & \left\{\psi_{h} \in W_{h^{-}} ;\left\|\psi_{h}\right\|_{W^{\prime}}=1\right\}
\end{array}
$$

Then there exists a constant $C$, depending only on $\mathrm{A}, \mathrm{B}, \alpha$, and $\beta$, such that for each pair of linear forms $f(\theta)$ on $W$ and $g(\Leftrightarrow)$ on $M$, if $(\phi, \lambda)$ satisfics $(10.1)$ and $\left(\phi_{h}, \lambda_{h}\right)$ satisfies $(10.5)$, we have

(1) The symbols $B$ and $B_{h}$ have been reserved for the restrictions of $\bar{B}$ to $V^{\perp}$ and of $\bar{B}_{h}$ to $V_{h}^{\perp}$, respectively. 


$$
\left.\left\|\phi-\phi_{h}\right\|_{W}=\left\|\lambda-\lambda_{h}\right\|_{M} \leqslant C \inf _{\psi_{h} \in W_{h}}\left\|\phi-\psi_{h}\right\|_{W}+\inf _{\mu_{h} \in M_{h}}\left\|\lambda-\mu_{h}\right\|_{M}\right\} .
$$

Proof. Let $V_{h}^{\perp}$ be the subspace of $W_{h}$ orthogonal to $V$. We introduce, as in the proof of Theorem 10.1, the operator $B_{h} \in \mathscr{L}\left(\mathrm{V}_{\mathrm{h}}^{\perp}, M\right)$ and its adjoint $B_{h}^{*} \in \mathscr{L}\left(\mathrm{M}, \mathrm{V}_{\mathrm{h}}^{\perp}\right)$. The discrete inf-sup condition (10.13) signifies that the operators $B_{\mathrm{h}}$ and $B_{h}^{*}$ are invertible or more precisely that we have

$$
\left\|B_{h}^{-1}\right\|_{\mathscr{L}\left(\mathrm{M}_{\mathrm{h}}, \mathrm{V}_{\mathrm{h}}^{\perp}\right)}=\left\|B_{\mathrm{h}}^{*}-1\right\|_{\mathscr{L}\left(\mathrm{V}_{\mathrm{h}}^{\perp}, \mathrm{M}_{\mathrm{h}}\right)} \leq \frac{1}{\beta}
$$

Similary the condition of $V_{h}$-ellipticity (10.12) implies that the operator $A_{h} \in$ $\mathscr{L}\left(\mathrm{V}_{h}, \mathrm{~V}_{h}\right)$ associated with the bilinear form $a(\cdot, \cdot)$ is invertible and that we have

$$
\left.\left\|A_{\mathrm{h}}^{-1}\right\|_{\mathscr{L}\left(\mathrm{V}_{\mathrm{h}}\right.}, \mathrm{V}_{\mathrm{h}}\right) \leq \frac{1}{\alpha}
$$

It is now easy to check that the bilinear form defined with the aid of (9.3) on $X \times X$, where $X=W \times M$ (and $Y=X$ ), satisfies (9.4) with a constant $K$ depending only on $A$ and $B$ and for the choice $X_{h}=W_{h} \times M_{h}$ (and $Y_{h}=X_{h}$ ) satisfies (9.6) with a constant $\kappa>0$ which depends only on $A, \alpha$ and $\beta$. An application of Theorem 9.3 then leads us to the estimation (10.14).

Remark 10.5 . This error estimate is optimal in the product space $\mathrm{X}=\mathrm{W} \times \mathrm{M}$. This does not mean that the estimation of a single term, $\left\|\phi-\phi_{h}\right\|_{W}$ for example, is necessarily optimal. Say for instance that $g(\cdot)=0$ and $V_{h}$ is a subspace of $V$. Then we obviously have

$$
\left\|\phi-\phi_{h}\right\|_{W} \leq C \inf _{\psi_{h} \in V_{h}} \quad\left\|\phi-\psi_{h}\right\|_{W}
$$

This sort of refinement of the theory has been developed by FALK \& OSBORN [1980]. We will point out such refinements in the examples treated hereafter.

Remark 10.6. We can deduce from Theorem 10.4 a convergence theorem similar to Theorem 9.4 for a family of pairs of subspaces $\left(W_{h}, M_{h}\right)$ indexed by $h \in H$. On the other hand we point out that the approximation result of Theorem 10.4 is still of interest if the chosen norms or even the spaces $W$ and $M$ themselves change with the parameter $h$ as will indeed be the case for hybrid methods. The error bound (10.14) holds with constant $C$ independent of $h$ as long as the constants $A, B, \alpha$ and $\beta$ may be taken independently of $h$. 
Remark 10.7 - Theorem 10.3 gives sufficient conditions for concluding that the inf-sup condition for $b(\cdot, \cdot)$ on $W \times M$ is inherited by $b(\cdot, \cdot)$ on $W_{h} \times M_{h}$. However, given a family of pairs of subspaces $\left(W_{h}, M_{h}\right)$ indexed by $h \in H$, to obtain convergence results (cf. Remark 10.6) we need to know that the discrete inf-sup condition holds uniformly in the sense that there exists $\beta$ independent of $h$ such that for each $h$ $\in H$

$$
\inf _{\left\{\mu_{h} \in M_{h} ;\left\|\mu_{h}\right\|_{M}=1\right\}} \sup _{\left\{\psi_{h} \in W_{h} ;\left\|\psi_{h}\right\|_{W}=1\right\}} b\left(\psi_{h}, \mu_{h}\right) \geq \beta>0
$$

This will be the case if the norms of $R_{h}$ and $\pi_{h}$ of Theorem 10.3 are independent of $h$.

Remark 10.8. With the methods used here to analyze numerically a problem with Lagrangian multipliers of the form (10.1) whose solution is approximated by the solution of a finite dimensional problem of the form (10.6), it is easy to find sufficient conditions for analyzing a problem of the following form : find $\phi$ and $\lambda$ satisfying

$$
\begin{cases}\phi \in W, \lambda \in M & \\ a(\phi, \psi)+b(\psi, \lambda)=f(\psi) & \text { for all } \psi \in W \\ b(\phi, \mu)+d(\lambda, \mu)=g(\mu) & \text { for all } \mu \in M .\end{cases}
$$

This generalization introduces a supplementary bilinear form $d(\cdot, \cdot)$ on $M \times M$ which we suppose is continuous, and we let $D$ be a constant such that

$$
\mathrm{d}(\lambda, \mu) \leq \mathrm{D}\|\lambda\|_{\mathrm{M}}\|\mu\|_{\mathrm{M}} \quad \text { for all } \lambda \in \mathrm{M} \text { and } \mu \in \mathrm{M}
$$

Given finite dimensional subspaces $W_{h} \subset W$ and $M_{h} \subset M$, the corresponding discrete problem associated with $(10.16)$ is written as follows : find $\phi_{h}$ and $\lambda_{h}$ satisfying

$$
\begin{cases}\phi_{h} \in W_{h}, \lambda_{h} \in M_{h}, & \\ a\left(\phi_{h}, \psi_{h}\right)+b\left(\psi_{h}, \lambda_{h}\right)=f\left(\psi_{h}\right) & \text { for all } \psi_{h} \in W_{h} \\ b\left(\phi_{h}, \mu_{h}\right)+d\left(\lambda_{h}, \mu_{h}\right)=g\left(\mu_{h}\right) . & \text { for all } \mu_{h} \in M_{h} .\end{cases}
$$

If in addition to the hypotheses of Theorem 10.4 we suppose that

$$
\begin{array}{ll}
\mathrm{a}\left(\psi_{\mathrm{h}}, \psi_{\mathrm{h}}\right) \geq 0 & \text { for all } \psi_{\mathrm{h}} \in \mathrm{W}_{\mathrm{h}}, \\
\mathrm{d}\left(\mu_{\mathrm{h}}, \mu_{\mathrm{h}}\right) \leq 0 & \text { for all } \mu_{\mathrm{h}} \in \mathrm{M}_{\mathrm{h}},
\end{array}
$$


and that one of the two forms $a(\cdot, \cdot)$ and $d(\cdot, \cdot)$ is symmetric, then problem (10.18) has a unique solution $\left(\phi_{h}, \lambda_{h}\right)$. Moreover, if $(\phi, \lambda)$ denotes the solution of $(10.16)$, an error bound analogous to that given by (10.14) may be obtained with a constant $C$ depending only on A, B, D, $\alpha$ and $\beta$. Another generalization of Theorem 10.4 is obtained by assuming in addition to the hypotheses of Theorem 10.4 that there is a positive number $\delta, 0<\delta \leq 1$, such that

$$
d\left(\lambda_{h}, \mu_{h}\right) \leq(1-\delta) \frac{\alpha}{\sqrt{\alpha^{2}+A^{2}}} \frac{\beta^{2}}{A}\left\|\lambda_{h}\right\|_{M}\left\|\mu_{h}\right\|_{M} \quad \text { for all } \lambda_{h} \in M_{h} \text { and } \mu_{h} \in M_{h} .
$$

The conclusion is the same as in the above generalization except that here the constant $\mathrm{C}$ depends also on $\delta$.

The demonstration of the first generalization relies on the fact that if $H$ is a Hilbert space with inner product $\left((,)_{\mathrm{H}}\right.$ and $U \in \mathscr{L}(\mathrm{H}, \mathrm{H})$ is positive in the sense that $((U \mathrm{x}, \mathrm{x}))_{\mathrm{H}} \geq 0$, for all $\mathrm{x} \in \mathrm{H}$, then $I+U$ is invertible in $\mathscr{L}(\mathrm{H}, \mathrm{H})$ and $\left\|(I+U)^{-1}\right\|_{\mathscr{L}(\mathrm{H}, \mathrm{H})} \leq 1$. To show that the second generalization is valid one uses that if $\mathrm{H}$ is a Hilbert space and $U \in \mathscr{L}(\mathrm{H}, \mathrm{H})$ has norm in $\mathscr{L}(\mathrm{H}, \mathrm{H})$ less than $1-\theta$ for some $\theta$ in $(0,1]$, then $I+U$ is invertible in $\mathscr{L}(\mathrm{H}, \mathrm{H})$ and $\left\|\left(I+U^{-1}\right)\right\|_{\mathscr{L}(\mathrm{H}, \mathrm{H})} \leq \frac{1}{\theta}$.

Remark 10.9. An extension of the above results of BREZZI [1974b] to the case of the following generalized saddle point problem :

$$
\begin{cases}(\phi, \lambda) \in W \times M, & \\ a(\phi, \psi)+b_{1}(\psi, \lambda)=f(\psi) & \text { for all } \psi \in W, \\ b_{2}(\phi, \mu) & \text { for all } \mu \in M,\end{cases}
$$

for which the bilinear forms $b_{1}(\cdot, \cdot)$ and $b_{2}(\cdot, \cdot)$ on $W \times M$ are distinct, can be deduced from the more extensive generalization given in NICOLAIDES [1982]. 


\section{1 - EFFECTS OF NUMERICAL INTEGRATION}

Under the hypotheses of Theorem 10.2, problem (10.6), the problem with Lagrangian multiplier, is reduced, once the bases of $W_{h}$ and of $M_{h}$ have been chosen, to the resolution of an invertible linear system of order $N$, with $N=\operatorname{dim} W_{h}+\operatorname{dim} M_{h}$. Several algorithms adapted to the resolution of such linear systems will be presented in Section 17. For the moment we remark only that even the formation of the linear system can turn out to be practically impossible or simply too expensive in calculation time. Thus we are led to replace problem (10.6) by the following problem : find $\left(\phi_{h}^{*}, \lambda_{h}^{*}\right)$ satisfying

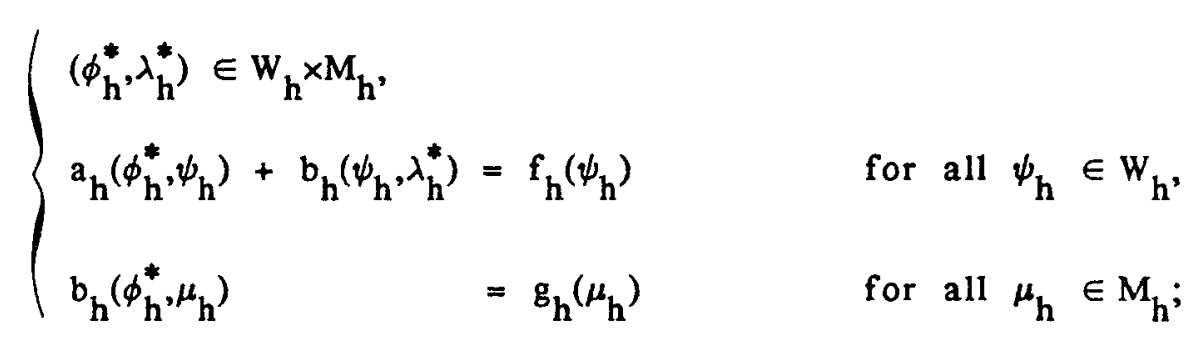

where $a_{h}(\cdot, \bullet)$ and $b_{h}(\cdot, \bullet)$ are bilinear forms on $w_{h} \times W_{h}$ and $w_{h} \times M_{h}$, respectively, and $f_{h}(\cdot)$ and $g_{h}(\cdot)$ are linear forms on $W_{h}$ and $M_{h}$, respectively. When $g_{h}(\cdot)=0$, if $\left(\phi_{h}^{*}, \lambda_{h}^{*}\right)$ is the solution of (11.1), then the first argument $\phi_{h}^{*}$ is a solution of the variational problem : find a solution $\phi_{h}^{*}$ such that

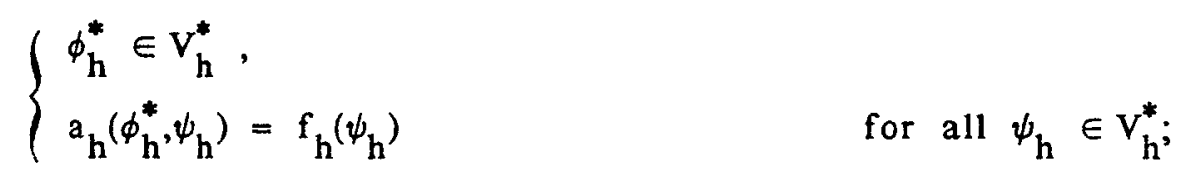

where the subspace $V_{h}^{*}$ is defined by

$$
\mathrm{V}_{\mathrm{h}}^{*}=\left\{\psi_{\mathrm{h}} \in \mathrm{W}_{\mathrm{h}} ; \forall \mu_{\mathrm{h}} \in \mathrm{M}_{\mathrm{h}}, \mathrm{b}_{\mathrm{h}}\left(\psi_{\mathrm{h}}, \mu_{\mathrm{h}}\right)=0\right\}
$$


Remark 11.1 For all of the examples that we shall consider, the physical parameters of the modelled problem actually play a role only in the expression of the bilinear form $a(\cdot, \cdot)$. The form $b(\cdot, \cdot)$ is independent of these parameters; the same is true, a fortiori, for the subspace $V$ defined by (10.3).

Futhermore, the spaces $W_{h}$ and $M_{h}$ are constructed in such a way that the exact calculation of the term $b\left(\psi_{h}, \mu_{h}\right)$ poses no practical difficulty since it always reduces to the calculation of an integral on an affine manifold of a polynomial function. In the following we shall point out (cf. Remark 11.2) where it can be of some interest to replace the bilinear form $b(\cdot, \cdot)$ by another $b_{h}(\cdot, \cdot)$.

Defining $X_{h}$ to be the product space $W_{h} \times M_{h}$ and putting

$$
\left\{\begin{array}{l}
\mathrm{k}_{h}\left(u_{h}, v_{h}\right)=a\left(\phi_{h}, \psi_{h}\right)+b\left(\psi_{h}, \lambda_{h}\right)+b\left(\phi_{h}, \mu_{h}\right) \\
\text { and } \\
f_{h}\left(v_{h}\right)=f_{h}\left(\psi_{h}\right)+g_{h}\left(\mu_{h}\right),
\end{array}\right.
$$

for $u_{h}=\left(\phi_{h}, \lambda_{h}\right)$ and $v_{h}=\left(\psi_{h}, \mu_{h}\right)$, we can write, as in Section 9, problem (11.1) in the form : find $u_{h}^{*}$ satisfying

$$
\left\{\begin{array}{l}
u_{h}^{*} \in x_{h}, \\
k_{h}\left(u_{h}^{*}, v_{h}\right)=\ell_{h}\left(v_{h}\right)
\end{array}\right.
$$$$
\text { for all } u_{h} \in X_{h} \text {. }
$$

Then in a slightly more general setting, this leads us to study the approximation of the solution $u$ of problem (9.2) by the solution $u_{h}^{*}$ of the following problem : find $u_{h}^{*}$ satisfying

$$
\left\{\begin{array}{l}
u_{h}^{*} \in x_{h}, \\
k_{h}\left(u_{h}^{*}, v_{h}\right)=l_{h}\left(v_{h}\right)
\end{array}\right.
$$$$
\text { for all } v_{h} \in Y_{h} \text {, }
$$ 
where $k_{h}(\cdot, \cdot)$ is a bilinear form on $X_{h} \times Y_{h}$ and $\ell_{h}(\cdot)$ is a linear form on $Y_{h}$, with $X_{h} \subset X$ and $Y_{h} \subset Y$. The analysis is based on the following result :

THEOREM 11.1. Suppose that $\mathrm{k}(\cdot, \cdot)$ is a bilinear form, continuous on $\mathrm{X} \times \mathrm{Y}$ and let $\mathrm{K}$ be a number such that (9.4) is satisfied. We suppose that the choice of subspaces $\mathrm{X}_{\mathrm{h}}$ and $\mathrm{Y}_{\mathrm{h}}$ is such that

$$
\operatorname{dim} Y_{h}=\operatorname{dim} X_{h}
$$

and that there exists a number $\kappa_{\mathrm{h}}>0$ such that

$$
\sup _{\left(v_{h} \in Y_{h} ;\left\|v_{h}\right\|_{Y}=1\right)} k_{h}\left(u_{h}, v_{h}\right) \geq \kappa_{h}\left\|u_{h}\right\|_{X} \quad \text { for all } u_{h} \in X_{h} .
$$

Then there exists a constant $\mathrm{C}$, depending only on $\mathrm{K}$ and $\kappa_{\mathrm{h}}$, such that if $\mathrm{u}$ is a solution of (9.2) and $\mathrm{u}_{\mathrm{h}}^{*}$ is the solution of (11.5), we have

$$
\begin{aligned}
\left\|u-u_{h}^{*}\right\|_{X} \leq & C \inf _{w_{h} \in X_{h}}\left(\left\|u-w_{h}\right\|_{X}+\sup _{v_{h} \in Y_{h}} \frac{a\left(w_{h}, v_{h}\right)-a_{h}\left(w_{h}, v_{h}\right)}{\left\|v_{h}\right\|_{Y}}\right)+ \\
& \left.+\sup _{v_{h} \in Y_{h}} \frac{\ell\left(v_{h}\right)-\ell_{h}\left(v_{h}\right)}{\left\|v_{h}\right\|_{Y}}\right\} .
\end{aligned}
$$

This is, of course, in the case $a_{h}(\cdot, \cdot)=a(\cdot, \cdot)$ and $\ell_{h}(\cdot)=\ell(\cdot)$, the result (9.7) given in Theorem 9.3. It generalizes the results of STRANG [1972] developed for the elliptic variational problem. The demonstration may be obtained by simply adapting the demonstration of Strang's result given by CIARLET [1977, pp.186187] for example.

We return now to the discrete problem (11.1), Theorem 10.4 may be generalized to the following result :

THEOREM 11.2. Suppose that $a(\cdot, \cdot)$ and $\mathrm{b}(\cdot, \cdot)$ are continuous bilinear forms on $\mathrm{W} \times \mathrm{W}$ and on $\mathrm{W} \times \mathrm{M}$ respectively, and let $\mathrm{A}$ and $\mathrm{B}$ be numbers such that (10.10) and (10.11) are satisfied. Let $\mathrm{A}_{\mathrm{h}}$ be a number such that

$$
a_{h}\left(\phi_{h}, \psi_{h}\right) \leq A_{h}\left\|\phi_{h}\right\|_{W}\left\|\psi_{h}\right\|_{W} \quad \text { for all } \phi_{h} \in W_{h} \text { and for all } \psi_{h} \in W_{h}
$$


Suppose further that there exists numbers $a_{h}>0$ and $\beta_{h}>0$ such that

$$
a_{h}\left(v_{h}, v_{h}\right) \geq \alpha_{h}\left\|v_{h}\right\|_{W}^{2}
$$

and

$$
\begin{array}{lll}
\inf & \text { sup } & b\left(\psi_{h}, \mu_{h}\right) \geq \beta_{h}
\end{array}
$$

Then there exists a constant $C$ dependent only on $\mathrm{A}, \mathrm{B}, \mathrm{A}_{\mathrm{h}}, \alpha_{\mathrm{h}}$ and $\beta_{\mathrm{h}}$ such that if $(\phi, \lambda)$ satisfies (10.1) and $\left(\phi_{\mathrm{h}}^{*}, \lambda_{\mathrm{h}}^{*}\right)$ is the solution of (11.1) then we have

$$
\begin{aligned}
& \left\|\phi-\phi_{h}^{*}\right\|_{W}+\left\|\lambda-\lambda_{h}^{*}\right\|_{M} \leq \\
& C\left\{\inf _{\psi_{h} \in W_{h}} \quad\left(\left\|\phi-\psi_{h}\right\|_{W}+\sup _{\eta_{h} \in W_{h}} \frac{a\left(\psi_{h}, \eta_{h}\right)-a_{h}\left(\psi_{h}, \eta_{h}\right)}{\left\|\eta_{h}\right\|_{W}}\right)\right. \\
& +\inf _{\mu_{h} \in M_{h}}\left(\left\|\lambda-\mu_{h}\right\|_{M}+\sup _{\eta_{h} \in W_{h}} \frac{b\left(\eta_{h}, \mu_{h}\right)-b_{h}\left(\eta_{h}, \mu_{h}\right)}{\left\|\eta_{h}\right\|_{W}}\right) \\
& \left.+\sup _{\eta_{h} \in W_{h}} \frac{f\left(\eta_{h}\right)-f_{h}\left(\eta_{h}\right)}{\left\|\eta_{h}\right\|_{W}}+\sup _{\nu_{h} \in M_{h}} \frac{g\left(\nu_{h}\right)-g_{h}\left(\nu_{h}\right)}{\left\|\nu_{h}\right\|_{M_{h}}}\right\} .
\end{aligned}
$$

We may obtain this result by applying Theorem 11.1. With the given hypotheses and the definition of the bilinear form $k_{h}(\cdot, \cdot)$ given in (11.4), condition (9.4) is satisfied with $K$ depending only on $A$ and $B$ and condition (11.6) is satisfied with $\kappa_{h}>0$ depending only on $A_{h}, \alpha_{h}$ and $\beta_{h}$. The detailed demonstration of this type of result was given by THOMAS [1977, Chapter I, Paragraph 3].

Remark 11.2. The replacement of the bilinear form $b(\cdot, \cdot)$ by the bilinear form $b_{h}(\cdot, \cdot)$ entails the replacement of the subspace $V_{h}$ defined by $(10.7)$ by the subspace $V_{h}^{*}$ defined by (11.3). The ellipticity hypothesis (11.9) can certainly be satisfied without (10.12)'s being satisfied, even in the case where $a_{h}(\cdot, \cdot)=a(\cdot, \cdot)$ on $W_{h} \times M_{h}$. Also, the discrete inf-sup condition (11.10) may hold without (10.13)'s holding. On the contrary, the converses are valid; (10.12) implies (11.9) and (10.13) implies (11.10). However, we stress that a careless choice of numerical integration technique can turn a well posed discrete problem of the form (10.6) into an ill posed discrete problem of the form (11.1). One should be aware that by replacing $b(\cdot, \cdot)$ by $b_{h}(\cdot, \cdot)$ one is actually changing the numerical method, and this must be done with some care. 


\section{CHAPTER IV}

\section{MIXED FINITE ELEMENT METHODS}

\section{2 - EXAMPLES OF PRIMAL MIXED METHODS FOR A DIRICHLET PROBLEM}

A primal mixed variational formulation of the model problem, find a solution $(\mathrm{p}, \mathrm{u})$ of the system (4.4) - (4.5) with the homogeneous Dirichlet boundary condition $u=0$ on $\Gamma$, is obtained by characterizing the pair $(p, u)$ as the solution of

$(12.1) \quad \begin{cases}(\underline{p}, u) \in\left(L^{2}(\Omega)\right)^{n} \times H_{0}^{1}(\Omega), & \\ a(\underline{p}, q)+b(\underline{q}, u)=0 & \text { for all } q \in\left(L^{2}(\Omega)\right)^{n}, \\ b(\underline{p}, v) & \text { for all } v \in H_{0}^{1}(\Omega),\end{cases}$

where

(12.2) $\quad a(\underline{p}, q)=\sum_{i, j=1}^{n} \int_{\Omega} A_{i j} p_{j} q_{i} d x$,

and

(12.3) $\quad b(q, v)=-\int_{\Omega} q \cdot \underset{\sim}{\operatorname{grad}} \mathrm{v} d x$

We are here in the context of Chapter III with

$$
\mathrm{W}=\left(\mathrm{L}^{2}(\Omega)\right)^{\mathrm{n}}, \quad\|\underline{\mathrm{q}}\|_{\mathrm{W}}=\|\underline{q}\|_{O, \Omega},
$$

and

(12.5) $\quad M=H_{0}^{1}(\Omega), \quad\|v\|_{M}=|v|_{1, \Omega}$. 
With the given hypotheses concerning the coefficients $a_{i j}$, the bilinear form $a(\cdot, \cdot)$ is continuous on $W \times W$ and $W$-elliptic with constant of ellipticity $\alpha=$ $\alpha_{d}$, cf. (4.8). The bilinear form $b(\cdot, \cdot)$ is continuous on $W \times M$ and we clearly have

$$
\left.|v|_{1, \Omega}=1 \quad \Rightarrow \quad \sup _{\{q \in W ; \| q} \|_{0, \Omega}=1\right\}
$$

Thus Theorem 10.1 guarantees that, for each $f \in L^{2}(\Omega)$, problem (12.1) has a unique solution.

In order to construct a primal mixed approximation to this solution, we introduce a finite dimensional subspace $W_{h}$ of $\left(L^{2}(\Omega)\right)^{n}$ and a finite dimensional subspace $M_{h}$ of $H_{0}^{1}(\Omega)$. From Theorems 10.2 and 10.4 we deduce:

THEOREM 12.1. Suppose that the subspaces $\mathrm{W}_{\mathrm{h}}$ and $\mathrm{M}_{\mathrm{h}}$ are compatible in the sense that the discrete inf-sup condition is satisfied:

$$
\left.\left.\beta_{h}=\inf _{\left\{v_{h} \in M_{h}\right.} ;\left|v_{h}\right|_{1, \Omega}=1\right\} \quad \sup _{\{h} \in W_{h} ;\left\|q_{h}\right\|_{0, \Omega}=1\right\} \quad b\left(q_{h}, v_{h}\right)>0 .
$$

Then the problem of finding a pair $\left(\mathrm{p}_{h}, u_{h}\right)$ satisfying

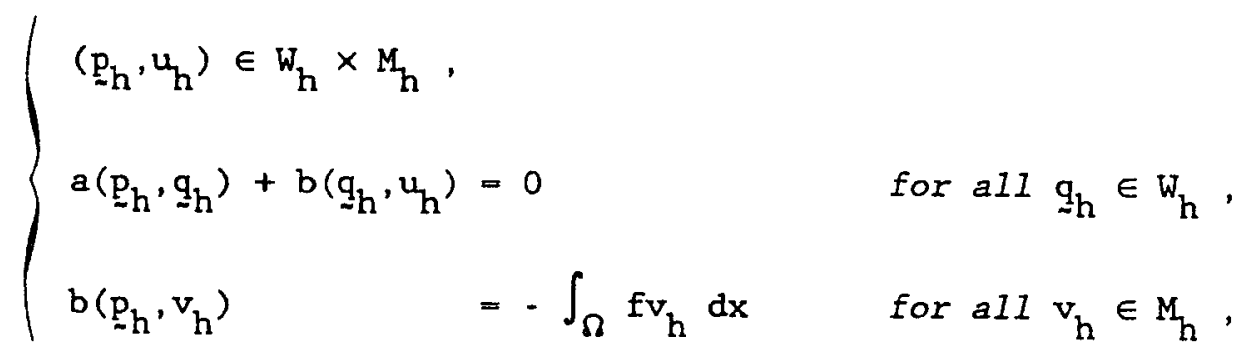

has a unique soiution. Furthermore, for each family of pairs of subspaces $\left(W_{h}, M_{h}\right)$ satisfying (12.7) uniformly in the sense that $\beta=$ inf $\beta_{h}>0$, there exists a constant $\mathrm{C}$, independent of $\mathrm{h}$, such that 
(12.9) $\left.\left\|\underset{\sim}{p}-\underline{p}_{h}\right\|_{0, \Omega}+\left|u-u_{h}\right|_{1, \Omega} \leq c \underset{q_{h} \in W_{h}}{\left\{\inf _{\underline{p}}-q_{-h}\right.} \|_{0, \Omega}+\underset{v_{h} \in \mathbb{M}_{h}}{\inf }\left|u-v_{h}\right|_{1, \Omega}\right\}$

Given a triangulation $T_{h}$ by $n$-simplexes $T$ of a domain $\bar{\Omega}$, supposed to be polyhedral, and a positive integer $k$, we take finite dimensional subspaces $W_{h}=W_{h}^{(k)}$ and $M_{h}=M_{h}^{(k)}$ given as follows :

$$
W_{h}=\left\{q \in\left(L^{2}(\Omega)\right)^{n} ; v T \in T_{h}, q_{T} \in\left(P_{k-1}(T)\right)^{n}\right\}
$$

and

$$
M_{h}=\left\{v \in H_{0}^{1}(\Omega) ; V T \in T_{h}, Y_{T} \in P_{k}(T)\right\}
$$

(With the notation (5.10) introduced in Chapter II we have $M_{h}=H_{0}^{1}(\Omega) \cap L_{h}^{(k)}$ ). Such a choice of subspaces is proposed in REDDY \& ODEN (1975); cf. also BABUSKA, ODEN, \& LEE (1977, Section 4).

THEOREM 12.2. For each positive integer $\mathrm{k}$, the subspaces $\mathrm{w}_{\mathrm{h}}$ and $\mathrm{M}_{\mathrm{h}}$ defined by $(12.10)$ and (12.11) satisfy the discrete inf-sup condition (12.7). Moreover, if $\left(T_{h} ; h \in H\right)$ is a regular family of triangulations, there is a constant $\mathrm{C}$, independent of $h$, such that if the solution $(\underset{\sim}{\mathrm{p}}, \mathrm{u})$ of $(12.1)$ belongs to $\left(\mathrm{H}^{\mathrm{k}}(\Omega)\right)^{\mathrm{n}} \times \mathrm{H}^{\mathrm{k}+1}(\Omega)$ we have the error estimate

$$
\left\|\underline{p}-\underline{p}_{h}\right\|_{0, \Omega}+\left|u-u_{h}\right|_{1, \Omega} \leq c h^{k}\left(\left.\left.\right|_{\sim} ^{p}\right|_{k, \Omega}+|u|_{k+1, \Omega}\right)
$$

In virtue of Theorem 12.1, the demonstration of Theorem 12.2 consists essentially of establishing the existence of a constant $\beta>0$ such that for each function $v_{h} \in M_{h}, v_{h} \notin 0$, there exists a function $q_{h} \in w_{h}, q_{h} \neq 0$, such that

$$
b\left(q_{h}, v_{h}\right) \geq \beta\left\|q_{h}\right\|_{0, \Omega}\left|v_{h}\right|_{1, \Omega}
$$

With the choice of subspaces given in (12.10) and (12.11), we clearly have that for each $v_{h} \in M_{h}$, grad $v_{h}$ belongs to $W_{h}$. The compatibility condition is 
thus satisfied with $\beta-1$. The proof is now terminated with an application of classical results giving error bounds for interpolation in $\left(L^{2}(\Omega)\right)^{n}$ and in $H_{0}^{1}(\Omega)$. (As $L_{h}^{(k)} u$ belongs to $M_{h}$, we may use Theorem 5.2).

Remark 12.1. When the coefficients $a_{i j}$ are sufficiently regular, for example when $a_{i j} \in C^{k}$, the regularity hypothesis $u \in H^{k+1}(\Omega)$ implies the regularity $\underset{\sim}{p} \in\left(H^{k}(\Omega)\right)^{n}$.

Remark 12.2. Even with no regularity hypothesis for the solution $(p, u)$ one can show that the primal mixed method applied in the context of Theorem 12.2 is convergent :

$$
\lim _{h \rightarrow 0} \underline{p}_{h}=\underline{p} \text { in }\left(L^{2}(\Omega)\right)^{n}, \quad \lim _{h \rightarrow 0} u_{h}=u \text { in } H_{0}^{1}(\Omega) ;
$$

cf. Remark 10.6 .

Remark 12.3. With $\mathrm{W}_{\mathrm{h}}=\mathrm{W}_{\mathrm{h}}^{(\mathrm{k})}$ as given in (12.10) and with $\mathrm{M}_{\mathrm{h}}=\mathrm{M}_{\mathrm{h}}^{\left(\mathrm{k}^{\prime}\right)}$ as given in (12.11) but with $k^{\prime}$ not necessarily equal to $k$, the compatibility hypothesis is satisfied once $k^{\prime} \leq k$. Error estimates optimal in $W \times M$ are obtained when $k^{\prime}=k$.

Remark 12.4 . Let $\hat{\mathrm{T}}$ be the reference $\mathrm{n}$-simplex and for each $\mathrm{T} \in T_{\mathrm{h}}$, let $\mathrm{F}_{\mathrm{T}}$ denote an affine bijection of $\hat{T}$ onto $T$ with Jacobien $J_{T}$. The spaces $W_{h}$ and $M_{h}$ given by (12.10) and (12.11) may also be defined as follows :

$(12.10$ bis $) W_{h}=\left(\underline{q} \in\left(L^{2}(\Omega)\right)^{n} ; V T \in T_{h}, J_{T} D F_{T}^{-1}\left(q_{T_{T}} F_{T}\right) \in\left(P_{k-1}(\hat{T})\right)^{n}\right)$,

(12.11 bis) $M_{h}=\left\{v \in H_{0}^{1}(\Omega) ; V T \in T_{h}, V_{T} \circ F_{T} \in P_{k}(\hat{T})\right\}$

It is with these definitions that the theory is extended to the case of curved finite elements where $T$ is the image of $\hat{T}$ under a bijection not required to be affine. However, we must note that for $v_{h} \in M_{h}$, grad $v_{h}$ need no longer belong to $W_{h}$. To show the compatibility relation in this case we introduce for $v_{h} \in M_{h}$, the function $\underline{q}_{h} \in W_{h}$ defined on each $T \in T_{h}$ by

$$
\left.\left(\underline{q}_{h}\right)_{T}\right) \circ F_{T}=\frac{1}{J_{T}}\left(D F_{T}\right)\left(D F_{T}\right)^{t}\left(\operatorname{grad} v_{h}{ }_{T}\right) \circ F_{T}
$$

and apply the relations $(6.23)$. 
These primal mixed methods may be easily adapted to the case in which $T_{h}$ is a triangulation by rectangles having sides parallel to the axes for $n=2$, by rectangular solids having faces parallel to the coordinate planes for $n=3$. For a regular family of such triangulations one may show :

THEOREM 12.3. (Stated here only for the case $\mathrm{n}=2, \mathrm{~T}$ a rectangle). For each positive integer $\mathrm{k}$, with $\mathrm{W}_{\mathrm{h}}=\mathrm{W}_{\mathrm{h}}^{(\mathrm{k})}$ and $\mathrm{M}_{\mathrm{h}}=\mathrm{M}_{\mathrm{h}}^{(\mathrm{k})}$ defined as follows:

$$
W_{h}=\left\{q \in\left(L^{2}(\Omega)\right)^{2} ; V T \in T_{h}, q_{T} \in P_{k-1, k}(T) \times P_{k, k-1}(T)\right\}
$$

and

$$
M_{h}=\left\{v \in H_{0}^{1}(\Omega) ; V T \in T_{h}, V_{T} \in P_{k, k}(T)\right\}
$$

the inf-sup condition, condition (12.7), is uniformly satisfied. Morever if the solution $(\underline{p}, \mathrm{u})$ of $(12.1)$ belongs to $\left(\mathrm{H}^{\mathrm{k}}(\Omega)\right)^{\mathrm{n}} \times \mathrm{H}^{\mathrm{k}+1}(\Omega)$ and $\left(\underline{\mathrm{p}}_{\mathrm{h}}, \mathrm{u}_{\mathrm{h}}\right)$ is the solution of (12.8) for $\mathrm{W}_{\mathrm{h}}$ and $\mathrm{M}_{\mathrm{h}}$ as in (12.13) and (12.14), there exists a constant $\mathrm{C}$, independent of $\mathrm{h}$, such that

$$
\left.\left\|\underline{p}-\underline{p}_{h}\right\|_{0, \Omega}+\left|u-u_{h}\right|_{1, \Omega} \leq\left. C h k{ }^{k}\right|_{k, \Omega}+|u|_{k+1, \Omega}\right) .
$$




\section{EXAMPLES OF DUAL MIXED METHODS FOR A DIRICHLET PROBLEM}

A dual mixed variational formulation of the model problem, find a solution $(\underline{p}, u)$ of system $(4.4)-(4.5)$ with the homogeneous Dirichlet boundary condition $u=0$ on $\Gamma$, is given by characterizing the pair $(\underline{p}, u)$ as the solution of

$$
\left\{\begin{array}{l}
(\underline{p}, u) \in H(\operatorname{div} ; \Omega) \times L^{2}(\Omega), \\
a(\underline{p}, q)+b(\underline{q}, u)=0 \\
b(\underline{p}, v)=-\int_{\Omega} f v d x
\end{array}\right.
$$$$
\text { for all } q \in H(\text { div; } \Omega) \text {, }
$$$$
\text { for all } \mathrm{v} \in \mathrm{L}^{2}(\Omega) \text {, }
$$

where

$$
a(p, q)=\sum_{i, j=1}^{n} \int_{\Omega} A_{i j} p_{j} q_{i} d x
$$

and

$$
b(\underline{q}, v)=\int_{\Omega} v \operatorname{div} \underline{q} d x
$$

We are again in the context of Chapter III with now

$$
W=H(\operatorname{div} ; \Omega), \quad\|q\|_{W}=\|q\|_{H(\operatorname{div} ; \Omega)},
$$

and

$$
M=L^{2}(\Omega), \quad\|v\|_{M}=\|v\|_{O, \Omega}
$$

The subspace $v$ of functions $q \in W$ for which $b(q, v)=0$ for $a i l v \in \bar{m}$ is none other than

$$
V=\{\underline{q} \in H(\operatorname{div} ; \Omega) ; \operatorname{div} q=0\}
$$

With the given hypotheses concerning the coefficients $a_{i j}$, the bilinear form $a(\cdot, \cdot)$ is continuous on $W \times W$ and $V$-elliptic with constant of ellipticity $\alpha=\alpha_{d}$, cf. (4.8). The bilinear form $b(\cdot, \cdot)$ is continuous on $W \times M$ and the inf-sup condition is satisfied : 
(13.7)

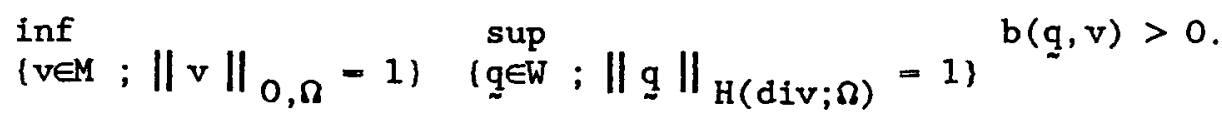

To establish (13.7) we shall use the auxillary problem. For each $v \in L^{2}(\Omega)$ denote by $\phi_{\mathrm{v}}$ the unique solution in $\mathrm{H}_{0}^{1}(\Omega)$, of $-\Delta \phi_{\mathrm{v}}-\mathrm{v}$ on $\Omega$. Then $q_{\mathrm{v}}=$ $\underset{\sim}{\operatorname{grad}} \phi_{\mathrm{v}}$ belongs to $\mathrm{H}(\operatorname{div} ; \Omega)$ and $\mathrm{b}\left(\underline{\mathrm{q}}_{\mathrm{v}}, \mathrm{v}\right)=\|\mathrm{v}\| \|_{0, \Omega}^{2}$. Since $\left\|\underline{q}_{\mathrm{v}}\right\|_{\mathrm{H}(\mathrm{div} ; \Omega)}$ $\leq\left\|\phi_{v} d_{1, \Omega}+\right\| v\left\|_{0, \Omega} \leq c\right\| v \|_{0, \Omega}$, inequality (13.7) follows. Thus we can apply Theorem 10.1 to obtain the existence and uniqueness of the solution $(p, u)$ of $(13.1)$.

In order to construct a dual mixed approximation of the solution $(\underline{p}, u)$ of (13.1), we introduce a finite dimensional subspace $W_{h}$ of $H(d i v ; \Omega)$ and a finite dimensional subspace $M_{h}$ of $L^{2}(\Omega)$. From Theorems 10.2 and 10.4 we deduce :

THEOREM 13.1. Suppose that the subspaces $\mathrm{W}_{\mathrm{h}}$ and $\mathrm{M}_{\mathrm{h}}$ are compatible in the sense that the discrete inf-sup condition is satisfied:

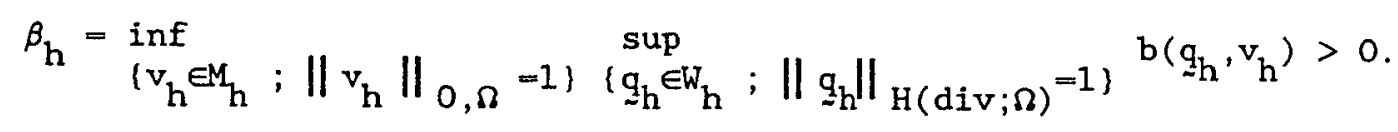

Then the problem of finding a pair $\left(\underline{p}_{h}, u_{h}\right)$ satisfying

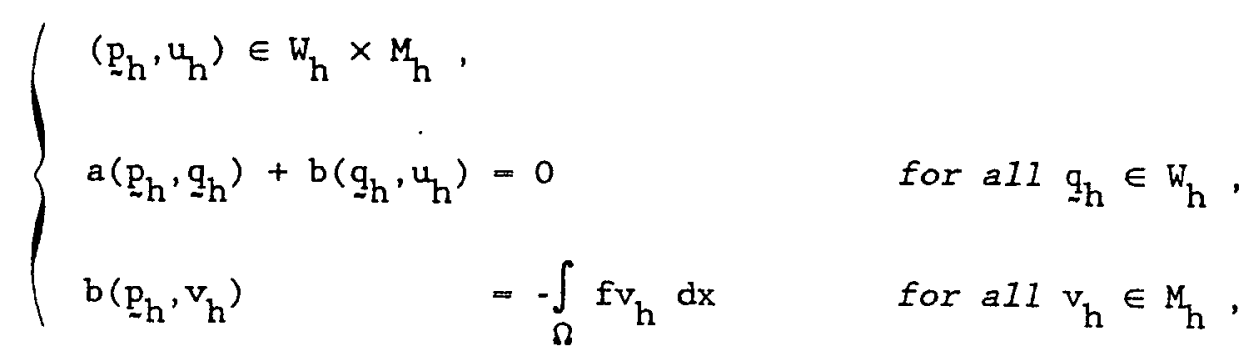

has a unique solution. Furthermore, for each family of pairs of subspaces $\left(\mathrm{W}_{h}, \mathrm{M}_{h}\right)$ such that for each $\mathrm{q}_{\mathrm{h}} \in \mathrm{W}_{\mathrm{h}}$ we have

$$
\left(\int_{\Omega} v_{h} \operatorname{div} q_{h} d x=0 \text {, for all } v_{h} \in M_{h}\right) \quad \Rightarrow \quad \operatorname{div} q_{h}=0
$$

and such that (13.8) is satisfied uniformly in the sense that $\beta=\inf \beta_{\mathrm{h}}>0$, there exists a constant $\mathrm{C}$, independent of $\mathrm{h}$, such that 


$$
\begin{aligned}
& \left\|\underline{p}-\underline{p}_{h}\right\|_{H(\operatorname{div} ; \Omega)}+\left\|u-u_{h}\right\|_{0, \Omega} \leq C\left(\inf _{q_{h} \in W_{h}}\left\|\underline{p}-q_{h}\right\|_{H(\operatorname{div} ; \Omega)}\right. \\
& \left.+\inf _{v_{h} \in M_{h}}\left\|u-v_{h}\right\|_{0, \Omega}\right) .
\end{aligned}
$$

Hypothesis (13.10) expresses the fact that the space $V_{h}$,

$$
v_{h}=\left\{q_{h} \in W_{h} ; v v_{h} \in M_{h}, b\left(q_{h} \cdot v_{h}\right)=0\right\}
$$

is a subspace of the space $V$ defined by (13.6). This hypothesis assures the uniform ellipticity of $a(\cdot, \cdot)$ on $V_{h}$, where the norm on $V_{h}$ is the $H(\operatorname{div} ; \Omega)$ norm.

Given a triangulation $T_{h}$ by $n$-simplexes $T$ of a domain $\bar{\Omega}$, supposed to be polyhedral, and a positive integer $k$, we take finite dimensional subspaces $W_{h}$ $=W_{h}^{(k)}$ and $M_{h}=M_{h}^{(k)}$ given as follows :

$$
W_{h}=\left\{\underline{q} \in H(\operatorname{div} ; \Omega) ; V T \in T_{h}, q_{T} \in D_{k}(T)\right\}
$$

and

$$
M_{h}=\left\{v \in L^{2}(\Omega) ; V T \in T_{h}, Y_{T} \in P_{k-1}(T)\right\}
$$

where $D_{k}(T)$ denotes the space of restrictions to $T$ of the functions in the space $D_{k}$ introduced in (6.5). (With the notation (6.17) introduced in Chapter II we have $W_{h}=E_{h}^{(k)}$ ). These examples were proposed and analyzed in RAVIART \& THOMAS (1977) in the 2 dimensional case and in NEDELEC (1979) in the 3-dimensional case. In the literature the space $w_{h} \times M_{h}$ or $w_{h}^{(k)} \times M_{h}^{(k)}$ is refered to as the Raviart-Thomas space of index $k$ (or sometimes $k-1$; cf. Remark (6.2)) or, in the 3-dimensional case, as the Raviart - Thomas Nedelec space.

THEOREM 13.2. For each positive integer $\mathrm{k}$, the subspaces $\mathrm{W}_{\mathrm{h}}$ and $\mathrm{M}_{\mathrm{h}}$ defined by (13.13) and (13.14) satisfy the discret inf-sup condition (13.8). Moreover, if $\left(T_{h} ; h \in H\right)$ is a regular family of triangulations, there is a constant $C$ independent of $h$ such that if the solution $(p, u)$ of (13.1) is such that $(\underline{\mathrm{p}}, \mathrm{u}) \in\left(\mathrm{H}^{\mathrm{k}}(\Omega)\right)^{\mathrm{n}} \times \mathrm{H}^{\mathrm{k}}(\Omega)$ and div $\underline{\mathrm{p}} \in \mathrm{H}^{\mathrm{k}}(\Omega)$ and if $\left(\mathrm{p}_{\mathrm{h}}, \mathrm{u}_{\mathrm{h}}\right)$ is the solution of (13.9) associated with (13.13) and (13.14), then we have the error estimate 
(13.15) $\left\|\underset{\sim}{\mathrm{p}-\mathrm{p}_{\mathrm{h}}}\right\|_{\mathrm{H}(\operatorname{div} ; \Omega)}+\left\|\mathrm{u}-\mathrm{u}_{\mathrm{h}}\right\|_{0, \Omega} \leq \mathrm{Ch}^{\mathrm{k}}\left(|\mathrm{u}|_{\mathrm{k}, \Omega}+|\underset{\sim}{\mathrm{p}}|_{k, \Omega}+|\operatorname{divp}|_{\mathrm{k}, \Omega}\right)$.

Proof . With $W_{h}$ and $M_{h}$ as given in (13.13) and (13.14) we clearly have that div $\underline{q}_{h} \in M_{h}$ for each $\underline{q}_{h} \in W_{h}$ and thus that $v_{h} \subset v$. To establish the discrete inf-sup condition we apply Theorem 10.3. Take for subspace of $W=H($ div $; \Omega)$ the space $W=\left(H^{1}(\Omega)\right)^{n}$ and for continuous linear operator $\pi_{h}$ from $W$ into $W_{h}$, the operator $\pi_{h}=E_{h}^{(k)}$, given by (6.2) together with (6.14). Then (6.15) guarantees that $(10.9)$ is satisfied. Let $R$ be any continuous linear map from $L^{2}(\Omega)$ into $\left(H^{1}(\Omega)\right)^{n}$ satisfying

$$
\operatorname{div}(\operatorname{Rv})=v,
$$

and let $R_{h}$ from $M_{h}$ into $W=\left(H^{1}(\Omega)\right)^{n}$ be defined by

$$
R_{h} v_{h}=R v_{h} \quad \text { for } a l l v_{h} \in M_{h}
$$

We note that with sufficient regularity the function $q_{-v} \in H(d i v ; \Omega)$ used to establish the inf-sup condition (13.7) belongs to $\left(\mathrm{H}^{1}(\Omega)\right)^{\mathrm{n}}$ and $\mathrm{Rv}$ could be taken to be $q_{\sim v}$. To avoid having to invoke regularity results for Dirichlet problems in polyhedral domains which would necessitate certain restrictions on the geometry of $\Omega$, it suffices to extend the functions $v \in L^{2}(\Omega)$ by zero to functions $\tilde{v} \in L^{2}(O)$ where $O$ is a ball containing $\Omega$, to solve the Dirichlet problem $\Delta \phi_{\mathrm{v}}=\tilde{\mathrm{v}}$ in $0, \phi_{\mathrm{v} / \partial O}=0$, and to define Rv to be the restriction to $\Omega$ of $\underset{\sim}{\operatorname{grad}} \phi_{\mathrm{v}}$.

To verify (10.8) we note there is a constant $C_{O}$ depending only on the ball $O$ containing $\Omega$, such that

$$
\left\|\mathrm{R}_{\mathrm{h}} \mathrm{v}_{\mathrm{h}}\right\|_{1, \Omega} \leq \mathrm{c}_{O}\left\|\mathrm{v}_{\mathrm{h}}\right\|_{0, \Omega} .
$$

Thus

$$
\frac{b\left(R_{h} v_{h}, v_{h}\right)}{\left\|R_{h} v_{h}\right\|_{W}}=\frac{\left\|v_{h}\right\|_{O, \Omega}^{2}}{\left\|R_{h} v_{h}\right\|_{1, \Omega}} \geq \frac{1}{c_{O}}\left\|v_{h}\right\|_{O, \Omega} .
$$


Since $\sup _{\left(\underline{\underline{W} W} ;\|\underline{\psi}\|_{W}=1\right)} ;\left(\underline{\psi}, v_{h}\right) \leq\|\operatorname{div} \underline{\psi}\|_{0, \Omega}\left\|v_{h}\right\|_{0, \Omega} \leq\left\|v_{h}\right\|_{0, \Omega}$. we have that $(10.8)$ holds with constant $\mathrm{C}=1 / \mathrm{C}_{O}$, and (13.8) now follows from Theorem 10.3.

Next suppose that $\left(T_{h} ; h \in H\right)$ is a regular family of triangulations. We have just seen that $R_{h}$ may be defined with norm independent of $h$, and Theorem 6.3 guarantees that for the operator $\pi_{h}=E_{h}^{(k)}$ there is a constant $C$ independent of $h$ such that

$$
\left\|\pi_{\mathrm{h}} \mathrm{q}\right\|_{\mathrm{H}(\operatorname{div} ; \Omega)} \leq \mathrm{C}\left(\mathrm{h}|\mathrm{q}|_{1, \Omega}+\|\operatorname{div} q\|_{0, \Omega}\right\}
$$

Thus the discrete inf-sup condition is satisfied uniformly and we have the error bound (13.11). Then to estimate the error of approximation

$\underset{\underline{q}_{h} \in W_{h}}{\inf }\left\|\underline{p}-q_{h}\right\|_{H(\operatorname{div} ; \Omega)}$, we take ${\underset{-q}{h}}_{h}=E_{h}^{(k)}(\underline{p})$ and use (6.19) and (6.20).

Remark 13.1. When the coefficients $a_{i j}$ are sufficiently regular, for example when $a_{i j} \in C^{k}$, the regularity hypothesis $\underset{\sim}{p} \in\left(H^{k}(\Omega)\right)^{n}$ and div $\underline{p}$ $\in H^{(k)}(\Omega)$ is enough to give the error bound (13.15). If we suppose only that p $\in\left(H^{k}(\Omega)\right)^{n}$ we may easily adapt the last part of the demonstration of Theorem 13.2 to obtain

$$
\left\|\underline{\sim}-\underline{p}_{h}\right\|_{H(\operatorname{div} ; \Omega)}+\left\|u-u_{h}\right\|_{0, \Omega} \leq c h^{k-1}\|\underline{p}\|_{k, \Omega} \text {, }
$$

or with a more careful study we obtain

$$
\left\|\underset{\sim}{p}-\underline{\sim}_{h}\right\|_{H(\operatorname{div} ; \Omega)} \leq \mathrm{Ch}^{k}\|\underline{p}\|_{k, \Omega}
$$

If in addition we suppose the regularity of the Dirichlet problem in $\Omega, a$ hypothesis which is satisfied once the open polyhedral domain $\Omega$ is convex, we have

$$
\left\|u-u_{h}\right\|_{0, \Omega} \leq C h^{k}\|u\|_{k^{*}, \Omega} \quad \text { with } k^{*}=\max (k, 2) \text {. }
$$

These results are demonstrated in FAIK \& OSBORN (1980, Section 3). 
Remark 13.2 . Even with no regularity hypothesis for the solution (p,u) one may show that the dual mixed method applied in the context of Theorem 13.2 is convergent :

$$
\lim _{h \rightarrow 0} \underline{p}_{h}=\underline{p} \quad \text { in } H(\operatorname{div} ; \Omega), \quad \lim _{h \rightarrow 0} u_{h}=u \quad \text { in } L^{2}(\Omega) .
$$

Remark 13.3 With $\mathrm{W}_{\mathrm{h}}=\mathrm{W}_{\mathrm{h}}^{(\mathrm{k})}$ as given in (13.13) and with $\mathrm{M}_{\mathrm{h}}=\mathrm{M}_{\mathrm{h}}^{\left(\mathrm{k}^{\prime}\right)}$ as given in (13.4) but with $k^{\prime}$ not necessarily equal to $k$, the compatibility hypothesis is satisfied once $k^{\prime} \leq k$. Error estimates, optimal in $W \times M$, are obtained when $k^{\prime}=k$. If $1 \leq k^{\prime}<k$ the subspace $V_{h}$ is no longer a subspace of $\mathrm{V}$; however, the method remains convergent.

Remark 13.4: let $\dot{T}$ be the reference $n$-simplex and for each $T \in T_{h}$, let $F_{T}$ denote an affine bijection of $\hat{T}$ onto $T$ with Jacobian $J_{T}$. The spaces $W_{h}$ and $M_{h}$ given by (13.13) and (13.14) may also be defined as follows:

$(13.13 \mathrm{bis}) \mathrm{W}_{\mathrm{h}}=\left\{\mathrm{q} \in \mathrm{H}(\mathrm{div} ; \Omega) ; \mathrm{v} \in \mathrm{T}_{\mathrm{h}},\left|\mathrm{J}_{\mathrm{T}}\right| \mathrm{DF}_{\mathrm{T}}^{-1}\left(q_{\mathcal{I}_{\mathrm{T}}} \circ \mathrm{F}_{\mathrm{T}}\right) \in \mathrm{D}_{\mathrm{k}}(\hat{\mathrm{T}})\right\}$

(13.14 bis) $M_{h}=\left\{v \in L^{2}(\Omega) ; V T \in T_{h}, V_{T} \circ F_{T} \in P_{k-1}(\hat{T})\right\}$

It is with these definitions that the theory is extended to the case of curved finite elements where $\mathrm{T}$ is the image of $\dot{\mathrm{T}}$ under a $\mathrm{C}^{1}$ bijection not required to be affine. To show the compatibility relation in this case, we construct for $v_{h} \in M_{h}$, the function $q_{h} \in W_{h}$ such that

$$
\operatorname{div} \mathrm{q}_{\mathrm{h}}=\theta_{\mathrm{h}} \mathrm{v}_{\mathrm{h}}
$$
where $\theta_{h} \in L^{\infty}(\Omega)$ is defined on each $T \in T_{h}$ by $\theta_{h}(x)=\sup _{y \in T} \frac{\left|J_{T}(y)\right|}{\left|J_{T}(x)\right|}$, for each
$x \in T$.

Remark 13.5. In the context of Theorem 13.2 it is obvious that for $q_{h} \in W_{h}$ we have div $q_{h} \in M_{h}$. Moreover, the proof of Theorem 13.2 shows that for each $v_{h} \in M_{h}$ there exists a function $q_{h}=\pi_{h} R_{h} v_{h} \in w_{h}$ such that div $q_{h}=v_{h}$. Thus in this case we have the characterization

$$
M_{h}=\left\{v_{h} \in L^{2}(\Omega) ; \exists q_{h} \in W_{h} \text { with div } q_{h}=v_{h}\right\}
$$


Such is not the case for the generalizations described in Remarks 13.3 and 13.4 .

For the dual mixed method analyzed above, given a triangulation $T_{h}$ of $\bar{\Omega}$ by $n$-simplexes, the vector functions in $W=H(d i v ; \Omega)$ are approximated in the space $W_{h}$ defined by (13.13) which is the space $E_{h}^{(k)}$ defined by (6.17) in Chapter 2. An alternative method is obtained by instead approximating the vector functions by functions in the space $E_{h}^{*}(k)$ also defined in Chapter 2 . For each integer $k, k \geq 2$, take $W_{h}=W_{h}^{(k)}$ and $M_{h}=M_{h}^{(k)}$ as follows :

$$
\begin{aligned}
& W_{h}=\left\{q \in H(\operatorname{div} ; \Omega) ; V T \in T_{h}, q_{T} \in D_{k}^{*}(T)\right\}, \\
& M_{h}=\left\{v \in L^{2}(\Omega) ; V T \in T_{h}, V_{T} \in P_{k-2}(T)\right\},
\end{aligned}
$$

where $D_{k}^{*}(T)=\left(P_{k-1}(T)\right)^{n} ;$ cf. (7.1). These approximation spaces were introduced and the corresponding methods analyzed in BREZZI, DOUGLAS \& MARINI (1985) in dimension $n=2$ and in BREZZI, DOUGLAS, DURAN \& FORTIN (1987) in dimension $n=3$. In the literature the space $W_{h} \times M_{h}$ or $W_{h}^{(k)} \times M_{h}^{(k)}$ is referred to as the Brezzi-Douglas-Marini space of index $k$ (or sometimes $k-1$, cf. Remark 6.2), or in the 3 dimensional case the Brezzi-Douglas-Duran-Fortin space.

As for the Raviart-Thomas (-Nedelec) spaces it is clear that if $p_{h} \in W_{h}$, then div $\underline{\sim}_{h} \in M_{h}$, and one may show that the discrete inf-sup condition (13.8) is satisfied. In fact we again have the characterization (13.19). For a regular Dirichlet problem in $\Omega$ we have the error estimates

$$
\begin{aligned}
& \left\|\underset{\sim}{\mathrm{p}}-\underline{\mathrm{p}}_{\mathrm{h}}\right\|_{0, \Omega} \leq C \mathrm{~h}^{\mathrm{k}}\|\underset{\mathrm{p}}{\mathrm{p}}\|_{\mathrm{k}, \Omega}, \\
& \left\|\mathrm{u}-\underline{\mathrm{w}}_{\mathrm{h}}\right\|_{0, \Omega} \leq C \mathrm{~h}^{\mathrm{k}-1}\|\mathrm{u}\|_{\mathrm{k}-1, \Omega}, \\
& \left\|\operatorname{div}\left(\underset{\sim}{\mathrm{p}-\underline{p}_{\mathrm{h}}}\right)\right\|_{0, \Omega} \leq C \mathrm{~h}^{\mathrm{k}-1}\|\operatorname{div} \underset{\sim}{\mathrm{p}}\| \|_{\mathrm{k}-1, \Omega} .
\end{aligned}
$$

For each of the dual mixed methods presented above for the case where $T_{\text {. }}$ is a triangulation by n-simplexes, there is a variant adapted to the case where $T_{h}$ is a triangulation by rectangles with sides parallel to the axes when $n=2$ or by rectangular solids with sides parallel to the coordinate 
planes when $n=3$. The description of these methods and the corresponding analyses may be found in the references cited above for the associated method for a triangulation by simplexes. Here we shall simply state for the case $n=2$ the analogue of Theorem 13.2 when $T_{h}$ is a triangulation of $\bar{\delta}$ by rectangles with sides parallel to the axes.

$\frac{\text { THEOREM 13.3 }}{(\mathrm{k})}:$ For each positive integer $\mathrm{k}$, the subspaces $\mathrm{W}_{\mathrm{h}}=\mathrm{W}_{\mathrm{h}}^{(\mathrm{k})}$ and $\mathrm{M}_{\mathrm{h}}=$ $\mathrm{M}_{\mathrm{h}}^{(\mathrm{k})}$ defined by

$$
W_{h}=\left\{q \in H(\operatorname{div} ; \Omega) ; V T \in T_{h}, q_{T} \in D_{k}^{D}(T)\right\}
$$

and

$$
M_{h}=\left\{v \in L^{2}(\Omega) ; V T \in T_{h}, v_{T} \in P_{k-1, k-1}(T)\right\}
$$

with $D_{k}^{\square}=P_{k, k-1} \times P_{k-1, k}$, satisfy the inf-sup condition (13.8). Moreover, if $\left(T_{\mathrm{h}} ; \mathrm{h} \in H\right)$ is a regular family of triangulations, there is a constant $C$, independent of $\mathrm{h}$, such that if the solution $(\underline{p}, \mathrm{u})$ of (13.1) is such that $(\underline{p}, u) \in\left(H^{k}(\Omega)\right)^{n} \times H^{k}(\Omega)$ and $\operatorname{div} \underset{p}{p} \in H^{k}(\Omega)$ and if $\left(\underline{p}_{h}, u_{h}\right)$ is the solution of (13.9) associated with (13.24) (13.25), we have the error estimate

$$
\left\|\underset{\sim}{p}-P_{h}\right\|_{H(\operatorname{div} ; \Omega)}+\left\|u-u_{h}\right\|_{0, \Omega} \leq \operatorname{Ch}^{k}\left(|u|_{k, \Omega}+|\underline{p}|_{k, \Omega}+|\operatorname{div} \underset{\sim}{p}|_{k, \Omega}\right) .
$$

Other dual mixed methods are presented and analysed in BREZZI, DOUGLAS, FORTIN, \& MARINI (1987) and in NEDELEC (1986); cf. Remark 7.2. 


\section{4 - EXAMPLES OF MIXED METHODS FOR A NEUMANN PROBLEM}

We consider the model problem, given $f \in L^{2}(\Omega)$ with $\int_{\Omega} f \mathrm{dx}=0$, find $u$ (to within a constant) and $p$ satisfying (4.4) and (4.5) with the homogeneous boundary condition $\underline{p} \cdot \underline{\nu}=0$ on $\Gamma$.

A primal mixed variational formulation of this problem is obtained by characterizing the pair $(\underline{p}, u)$ as the solution of

$(14.1) \begin{cases}(\underline{p}, u) \in\left(L^{2}(\Omega)\right)^{n} \times H^{1}(\Omega) / R, \\ a(\underline{p}, q)+b(q, u)=0 & \text { for all } q \in\left(L^{2}(\Omega)\right)^{n}, \\ b(\underline{p}, v) & =-\int_{\Omega} f v d x\end{cases}$

where the bilinear forms $a(\cdot, \cdot)$ and $b(\cdot, \cdot)$ are given by (12.2) and (12.3). Recalling the notation for the abstract problem of Section 10 , here we put

$$
\mathrm{W}=\left(\mathrm{L}^{2}(\Omega)\right)^{\mathrm{n}}, \quad \quad\|\mathrm{q}\|_{\mathrm{W}}=\|\mathrm{q}\|_{0, \Omega}
$$

and

$$
M=H^{1}(\Omega) / R, \quad \quad\|v\|_{M}=|v|_{1, \Omega^{\circ}}
$$

With $W_{h}$ and $M_{h}$ denoting finite dimensional subspaces of $\left(L^{2}(\Omega)\right)^{n}$ and $H^{1}(\Omega) / R$, respectively, we obtain the analogue of Theorem 12.1 , with no modification of the statement, and also the analogue of Theorem 12.2 if we specify the subspaces $W_{h}$ and $M_{h}$ as follows :

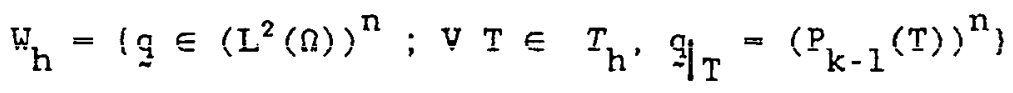

and

$$
M_{h}=\left(v \in H^{1}(\Omega) / R ; V T \in T_{h}, V_{T} \in P_{k}(T)\right)
$$

for some $k \geq 1$. In the case of a triangulation by rectangles, with the appropriate modification of the subspaces $W_{h}$ and $M_{h}$, the analogue of Theorem 12.3 is obtained. 
Thus for primal mixed methods there is no difficulty in adapting the theory for the Dirichlet problem to obtain that for the Neumann problem. The construction of examples may even be easier for the Neumann problem as the functions of $\mathrm{M}_{h}$ need no longer satisfy, a priori, the zero trace condition on $\Gamma$. The situation for dual mixed methods is quite the opposite.

A dual mixed variational formulation of the homogeneous Neumann problem

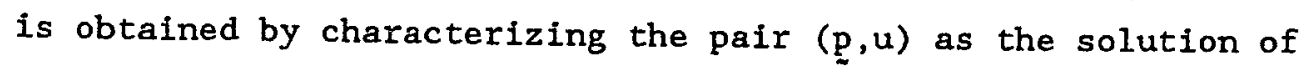

$$
\left\{\begin{array}{l}
(\underline{p}, u) \in H_{0}(\operatorname{div} ; \Omega) \times L^{2}(\Omega) / R \\
a(\underline{p}, q)+b(\underline{q}, u)=0 \\
b(\underline{p}, v) \quad=-\int_{\Omega} f v \cdot d x
\end{array}\right.
$$$$
\text { for all } q \in H_{0}(\operatorname{div} ; \Omega) \text {, }
$$$$
\text { for all } v \in L^{2}(\Omega) / R \text {, }
$$

where the bilinear forms $a(\cdot, \cdot)$ and $b(\cdot, \cdot)$ are, given by (13.2) and (13.3). For convenience, $L^{2}(\Omega) / R$ will be identified with the subspace of functions $v$ $\in L^{2}(\Omega)$ such that $\int_{\Omega} v d x=0$. Here the spaces $W$ and $M$ and their norms are defined by

$$
W=H_{0}(\operatorname{div} ; \Omega)
$$

$$
\|\mathrm{q}\|_{\mathrm{W}}=\|\mathrm{q}\|_{\mathrm{H}(\operatorname{div} ; \Omega)}
$$

and

$$
M=\left\{v \in L^{2}(\Omega) ; \int_{\Omega} v d x=0\right\}, \quad\|v\|_{M}=\|v\|_{0, \Omega} \text {. }
$$

The inf-sup condition analogous to (13.7) is also established with the aid of the dual problem. For each $v \in M$ denote by $\phi_{V}$ the unique element of $H^{1}(\Omega) / R$ satisfying $\Delta \phi_{\mathrm{v}}=\mathrm{v}$ in $\Omega$ and $\frac{\partial \phi_{\mathrm{v}}}{\partial \nu}=0$ on $\Gamma$. The function $\mathrm{q}_{\mathrm{v}}=\operatorname{grad}_{\sim} \phi_{\mathrm{v}}$ belongs to $W$ and satisfies div $q_{-v}=v$ in $\Omega$.

With $W_{h}$ and $M_{h}$ denoting finite dimensional subspaces of $H_{0}($ div; $\Omega$ ) and $M$ respectively, a demonstration similar to that given for Theorem 3.1 shows that if the discret inf-sup condition is satified, there exists a unique solution of the following problem : find $\left(\underline{p}_{h}, u_{h}\right)$ satisfying 


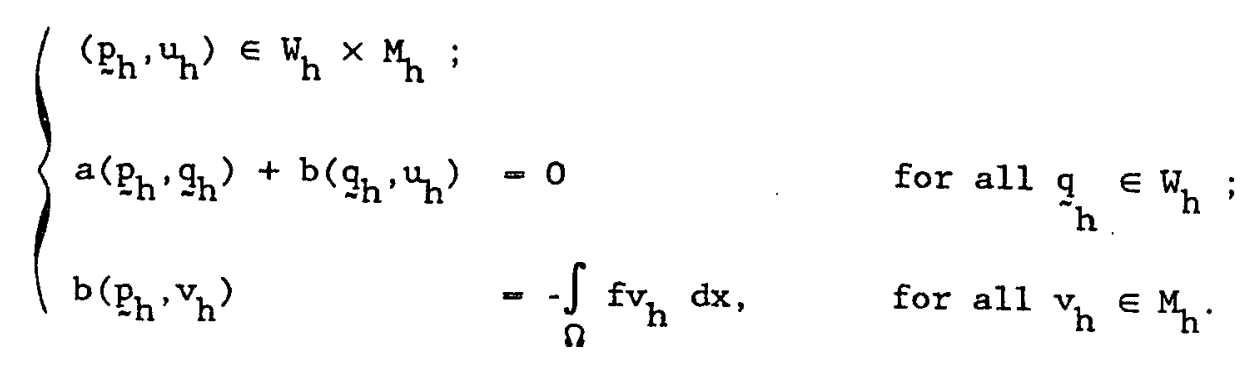

To show that the discret inf-sup condition holds for a pair of subspaces $W_{h}$ and $\mathrm{M}_{h}$ ' however, is a more delicate matter here than for the Dirichlet problem. It suffices, of course, to be able to associate to each $v_{h} \in M_{h} a q_{h}$ $\epsilon W_{h}$ with div $g_{h}=v_{h}$, but this is made more complicated here by the requirement that $\mathrm{q}_{h} \in \mathrm{w}_{\mathrm{h}} \subset \mathrm{W}$ have a vanishing normal trace on $\Gamma$. Nonetheless, one may show that the discrete inf-sup condition is satisfied when, for a triangulation $T_{h}$ by $n$-simplexes, the subspaces $W_{h}$ and $M_{h}$ are defined as follows :

$$
W_{h}=\left\{q \in H_{0}(\operatorname{div} ; \Omega) ; V T \in T_{h}, q_{T} \in D_{k}(T)\right\}
$$

and

$$
M_{h}=\left\{v \in L^{2}(\Omega) ; \int_{\Omega} v d x=0 \text { and } v T \in T_{h}, v_{T} \in P_{k-1}(T)\right\}
$$

for some positive integer $k$. We give the demonstration for the case $k=1$ :

Let $v_{h} \in M_{h}$; thus $v_{h}$ is constant on each $n$-simplex $T$ and has zero average over $\Omega$. Denote by $\Phi_{h}$ the space of functions $\phi_{h} \in H^{1}\left(T_{h}\right)$ with $\int_{\Omega} \phi_{h} d x=0$, with $\phi_{h / T}$, for each $T \in T_{h}$, an affine function, and with $\phi_{h}$ continuous across the interior faces at the barycenters of the faces $T^{\prime}$ common to two n-simplexes of $T_{h}$. For each $v_{h} \in M_{h}$, introduce the non-conforming solution $\phi_{h}\left(v_{h}\right)$ of the dual Neumann problem : find $\phi_{h}$ satisfying

$$
\left\{\begin{array}{l}
\phi_{\mathrm{h}} \in \Phi_{\mathrm{h}} \\
\sum_{\mathrm{T} \in T_{\mathrm{h}}} \int_{\mathrm{T}} \operatorname{grad} \phi_{\mathrm{h}} \cdot \underset{\sim}{\operatorname{grad}} \psi_{\mathrm{h}} \mathrm{dx}=\int_{\Omega} v_{\mathrm{h}} \psi_{\mathrm{h}} \mathrm{dx}, \quad \text { for all } \psi_{\mathrm{h}} \in \Phi_{\mathrm{h}}
\end{array}\right.
$$


Let $q_{h}=q_{h}\left(v_{h}\right)$ be the function of $\left(L^{2}(\Omega)\right)^{n}$ whose restriction to $T$, for each $T \in T_{h}$, coincides with the function of $D_{1}(T)$ having normal trace on $\partial T$ equal to that of $\left.\phi_{h}\right|_{T}$. One can verify that $q_{h}$ belongs to $W_{h}$ and that div $q_{h}=v_{h}$. One may further establish that, for a uniformly regular family of triangulations $\left(T_{h} ; h \in H\right)$, there exists a constant $C$, independent of $h$, such that

$$
\left\|\psi_{\mathrm{h}}\right\|_{O, \Omega} \leq \mathrm{C}\left(\sum_{\mathrm{T} \in T_{\mathrm{h}}}\left|\psi_{\mathrm{h}}\right|_{1, \mathrm{~T}}^{2}\right\}^{\frac{3}{2}},
$$

a result which amounts to a generalization of the Friedrichs-Poincaré inequalities to the nonconforming case. One thus obtains the existence of a (new) constant $C$, independent of $h$, such that for the preceding construction we have

(14.14) $\quad\left\|q_{h}\left(v_{h}\right)\right\|_{H(\operatorname{div} ; \Omega)} \leq c\left\|v_{h}\right\|_{O, \Omega}$,

thereby showing that the discrete inf-sup condition is satisfied uniformly. Hence we may deduce that there is a constant $C$, independent of $h$, such that

$$
\left\|\underline{p}-\underline{p}_{h}\right\|_{H(\operatorname{div} ; \Omega)}+\left\|u-u_{h}\right\|_{0, \Omega} \leq \operatorname{Ch}\left\{|u|_{1, \Omega}+|\underset{\sim}{p}|_{1, \Omega}+|\operatorname{div} p|_{1, \Omega}\right\}
$$

To verify the discrete inf-sup condition we have avoided using the regularity of the Neumann problem on polyhedral domains. Thus the above argument may be extended to the case of a problem with a Dirichlet condition on part of the boundary and a Neumann condition on the remaining part. For this type of problem, an extension of the above result to the case $k>1$ may be found in RAVIART \& THOMAS (1977a).

The uniform regularity hypothesis for the family of triangulations can be removed using the regularity of the solution of the Neumann problem for the Laplace equation in a polyhedral domain; cf. THOMAS (1987b). 


\section{CONDITION}

In this section we discuss the adaptation of mixed formulations to the following model problem : for a given function $f \in L^{2}(\Omega)$ and function $q \in$ $I^{2}(\Gamma)$, find a solution $(\underline{\sim}, u)$ of system (4.4) - (4.5) with the Robin's boundary condition

(15.1) $\quad \underline{p} \cdot \underline{\nu}+t u=g \quad$ on $\Gamma$

where $t$ is a continuous, strictly positive function on $\Gamma$. (Thus $t$ is bounded above and away from 0. )

A primal, mixed, variational formulation of this problem is obtained by characterizing the pair $(\underset{p}{p}, u)$ as the solution of

(15.2) $\begin{cases}(\underline{p} ; u) \in\left(L^{2}(\Omega)\right)^{n} \times H^{1}(\Omega), & \\ a(\underline{p}, q)+b(q, u)=0 & \text { for all } q \in\left(L^{2}(\Omega)\right)^{n}, \\ b(\underline{p}, v)+d(u, v)=-\int_{\Omega} f v d x-\int_{\Gamma} g v d \sigma & \text { for all } v \in H^{1}(\Omega),\end{cases}$

where the bilinear forms $a(\cdot, \cdot)$ and $b(\cdot, \cdot)$ are defined as for the Dirichlet and Neumann problems by $(12.2)$ and $(12.3)$ and where the bilinear form $d(\cdot, \cdot)$ is defined on $\mathrm{H}^{1}(\Omega) \times \mathrm{H}^{1}(\Omega)$ by

(15.3) $d(u, v)=-\int_{\Gamma}$ tuv do.

Problem (15.2) is the first example we have encountered of the generalization (10.16) developed in the abstract setting in Remark 10.7 with here

$(15.4) \quad W=\left(L^{2}(\Omega)\right)^{n}$ 
and

(15.5)

$$
M=H^{1}(\Omega)
$$

The numerical analysis of a primal mixed finite element method derived from formulation (15.2) is not difficult when to a triangulation $T_{h}$ of $\bar{\Omega}$ by $n$-simplexes $\mathrm{T}$ are associated the finite dimensional subspaces

$$
W_{h}=\left(q \in\left(L^{2}(\Omega)\right)^{n} ; v T \in T_{h}, q_{T} \in\left(P_{k-1}(T)\right)^{n}\right)
$$

and

$$
M_{h}=\left\{v \in H^{1}(\Omega) ; V T \in T_{h}, V_{T} \in P_{k}(T)\right\}
$$

where $k$ is a positive integer. We remark nonetheless that in this situation the inf-sup condition can never be satisfied as the functions constant on each $\mathrm{n}$-simplex belong to $\mathrm{M}_{\mathrm{h}}$.

With the dual mixed variational formulation, the solution $(\underline{p}, u)$ of system (4.4) - (4.5) with boundary condition (15.1) is characterized as the solution of

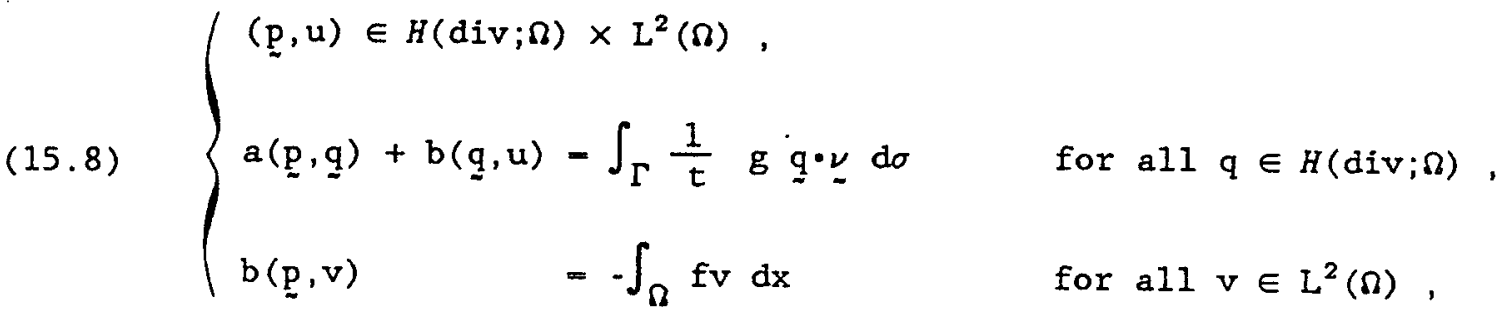

where the bilinear form $b(\cdot, \cdot)$ is defined as for the Dirichlet and Neumann problems by $(13.3)$ and where the bilinear form $a(\cdot, \cdot)$ is now given by

$$
a(\underline{p}, q)=\sum_{i, j=1}^{n} \int_{\Omega} A_{i j} p_{j} q_{i} d x+\int_{\Gamma} \frac{1}{t} \underline{p} \cdot \nu q \cdot \nu d \sigma .
$$

We thus obtain a problem of form (10.1) with now 
(15.10)

$$
\mathrm{W}-H(\operatorname{div} ; \Omega)
$$

and

$$
M=L^{2}(\Omega)
$$

There exists a continuous linear operator $\mathbf{R}$ from $\mathrm{L}^{2}(\Omega)$ into $H(\operatorname{div} ; \Omega)$ satisfying $\operatorname{div}(R v)=v$ for each $v \in L^{2}(\Omega)$. (One may use, for example, the operator $R$ from $L^{2}(\Omega)$ into $\left(H^{1}(\Omega)\right)^{n}$ constructed in the proof of Theorem 13.2). Hence the inf-sup condition is satisfied, the norms for the spaces $W$ and $M$ being given by $\|\cdot\|_{W}=\|\cdot\|_{H(\operatorname{div} ; \Omega)}$ and $\|\cdot\|_{M}=\|\cdot\|_{0, \Omega}$.

For a triangulation $T_{h}$ of $\Omega$ by $n$-simplexes $T$, take for finite dimensional subspaces $W_{h}$ and $M_{h}$

$$
W_{h}=\left\{\underline{q} \in H(\operatorname{div} ; \Omega) ; V T \in T_{h}, q_{T} \in D_{k}(T)\right)
$$

and

$$
M_{h}=\left\{v \in L^{2}(\Omega) ; V T \in T_{h}, V_{T} \in P_{k-1}(T)\right\},
$$

where $k$ is a positive integer. One can show with no new difficulties beyond those already treated for the problem with Dirichlet boundary condition that the problem, find a pair $\left({\underset{\sim}{h}}_{h}, u_{h}\right)$ satisfying

$$
\left\{\begin{array}{ll}
\left(\underline{p}_{h}, u_{h}\right) \in w_{h} \times \underline{M}_{h}, & \\
a\left(\underline{p}_{h}, q_{h}\right)+b\left(\underline{q}_{h}, u_{h}\right)=\int_{\Gamma} \frac{1}{t} g \underline{q}_{h} \cdot \nu d o & \text { for all } \underline{q}_{h} \in w_{h} \\
b\left(\underline{p}_{h}, v_{h}\right)=-\int_{\Omega} f v_{h} d x & \text { for all } v_{h} \in M_{h}
\end{array},\right.
$$

admits a unique solution $\left(\underline{p}_{h}, u_{h}\right)$. If $\left\{T_{h} ; h \in H\right)$ is a regular family of triangulations, the discrete inf-sup condition is satisfied uniformly and if further the solution $(p, u)$ is sufficiently regular, then we have

$$
\left\|\underline{p}-\underline{p}_{h}\right\|_{H(\operatorname{div} ; \Omega)}+\left\|\mathrm{u}-u_{h}\right\|_{0, \Omega}=O\left(h^{k}\right)
$$


All of the examples presented above treat only elliptic problems governed by second order partial differential equations of the form

$$
-\sum_{i, j=1}^{n} \frac{\partial}{\partial x_{i}}\left(a_{i j} \frac{\partial u}{\partial x_{j}}\right)=f
$$

The generalization of these methods to problems governed by an equation of the form

$$
-\sum_{i, j=1}^{n} \frac{\partial}{\partial x_{i}}\left(a_{i j} \frac{\partial u}{\partial x_{j}}\right)+\sum_{j=1}^{n} b_{j} \frac{\partial u}{\partial x_{j}}+c u=f
$$

poses no difficulty for the primal mixed formulation. The case is quite different for the dual mixed formulation. A mathematical analysis of this problem can be found in DOUGLAS \& ROBERTS (1982) and (1985). A generalization of these methods to the quasilinear problem where the coefficients in the governing equation depend on $u$ is analyzed in MILNER (1985). Several methods adapted to treat numerically the case where the the convection term is large in comparison with the diffusion term have been studied in JAFFRE (1984), JAFFRE \& ROBERTS (1985), JOLY (1982), and THOMAS (1987a). Another generalization of mixed methods to problems governed by an equation of the form

$$
-\sum_{i, j=1}^{n} \frac{\partial}{\partial x_{i}}\left(a_{i j} \frac{\partial u}{\partial x_{j}}\right)=f
$$

with coefficients $a_{i j}$ which can degenerate has been studied by LE ROUX (1982).

Estimations of the error made by a mixed method approximation have been given in norms other than the $\mathrm{L}^{2}$-norm and the $\mathrm{H}$ (div)-norm. Estimates for $\mathrm{u}-\mathrm{u}_{\mathrm{h}}$ in the $L^{\infty}$-norm have been given in SCHOLZ (1977), (1983), DOUGLAS \& ROBERTS (1985), and GASTALDI \& NOCHETTO (1987). Negative norm estimates in $\mathrm{H}^{-\mathrm{s}}(\Omega)$, for $s$ a nonnegative integer, are given for $u-u_{h}, \underline{p}-\underline{p}_{h}$, and $\operatorname{div}\left(\underline{p}-\underline{p}_{h}\right)$ in DOUGLAS \& ROBERTS (1985). These estimates have been exploited in DOUGLAS \& MILNER (1985) to obtain super convergence results. 
Mixed methods have been "generalized" to $4^{\text {th }}$ order problems such as those governed by an equation of the form $\Delta^{2} u=f$, where we write "generalized" with quotation marks to indicate that these methods were introduced, for the most part, before those presented for the $2^{\text {nd }}$ order problem and without the use of the general abstract theory for problems with Lagrangian multipliers. We cite in particular the work of BRAMBLE \& FALK (1983) ; BREZZI \& RAVIART (1978) ; CIARLET \& RAVIART (1974), HELLAN (1967); HERRMANN (1967), (1983); JOHNSON (1973) ; MERCIER (1974) ; MIYOSHI (1973); QUARTERONI (1980a), SCAPOLLA (1980); and SCHOLZ (1978). A precise analysis of the best known of these methods can be found in FALK \& OSBORN (1980). An excellent exposition is also given in CIARLET (1978 Chapter 7).

A generalization to problems governed by an equation of the form $(-\Delta)^{m} u=f$ for an integer $m, m \geq 2$ is proposed in BRAMBLE \& FALK (1985).

\section{7 - SOLUTION OF THE LINEAR SYSTEM}

We conclude this chapter with some remarks concerning the solution of the algebraic system resulting from a mixed method or, in particular, from a dual mixed method. We shall restrict our attention to the case of the model problem (4.1), (4.2) as in Sections 12 through 14. We assume that the continuous problem is written as a problem with Lagrangian multipliers in the form (8.1), and that finite dimensional subspaces $W_{h}$ and $M_{h}$ of the spaces $W$ and $M$ where the solution is sought have been introduced in such a way that $i$ ) and $i i)$ of Theorem 10.2 are satisfied. We thus suppose that the finite dimensional problem is of the form $(10.6)$ : find $\left(\psi_{h}, \lambda_{h}\right)$ satisfying

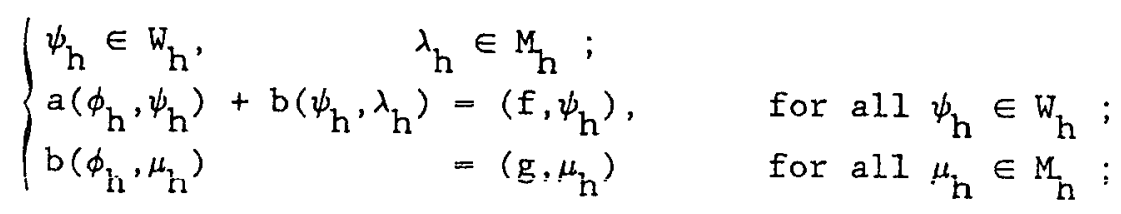

and we further suppose that $a(\cdot, \cdot)$ is symmetric. Once bases $W_{h}$ and $M_{h}$ have been chosen we may write the algebraic problem associated with (17.1) as

$$
\left(\begin{array}{cc}
A & B^{*} \\
B & 0
\end{array}\right)\left(\begin{array}{l}
\phi_{h} \\
\lambda_{h}
\end{array}\right)=\left(\begin{array}{c}
f_{h} \\
g_{h}
\end{array}\right) \text {, }
$$

where $A$ is a symmetric, dim $W_{h} \times \operatorname{dim} W_{h}$ matrix such that $\left(A \psi_{h}, \psi_{h}^{\prime}\right)=a\left(\psi_{h}, \psi_{h}^{\prime}\right)$ 
for all $\left(\psi_{h}, \psi_{h}^{\prime}\right)$ in $W_{h} \times W_{h}$, and $B$ is a dim $M_{h} \times \operatorname{dim} W_{h}$ matrix such that $\left(B \psi_{h}, \mu_{h}\right)=b\left(\psi_{h}, \mu_{h}\right)$ for all $\left(\psi_{h}, \mu_{h}\right)$ in $W_{h} \times M_{h}$. The functions $f_{h}$ and $g_{h}$ are the orthogonal projections of $f$ onto $W_{h}$ and $g$ onto $M_{h}$, respectively.

Theorem 10.2 guarantees that the matrix $L$,

$$
L=\left(\begin{array}{ll}
A & B^{*} \\
B & 0
\end{array}\right)
$$

of order $N \times N, N=\operatorname{dim} W_{h} \operatorname{dim} M_{h}$, is invertible; however, it is not positive definite. Hence direct solution of the system (17.2) is generally not feasible. On the other hand $A$ is here positive definite. So, theoretically it is always possible to eliminate $\phi_{h}$ from the system,

$$
\phi_{h}=\mathbf{A}^{-1}\left(f_{h}-B^{*} \lambda_{h}\right) \text {, }
$$

to obtain

$$
\mathrm{BA}^{-1} \mathrm{~B}^{*} \lambda_{\mathrm{h}}=-\mathrm{g}_{\mathrm{h}}+\mathrm{BA}^{-1} \mathrm{f}_{\mathrm{h}} \text {. }
$$

However, there is no reason, a priori, why $A^{-1}$ should be sparse.

For the primal mixed method, the space $W$ is $\left(L^{2}(\Omega)\right)^{n}$. So, among the degrees of freedom determining an element of $W_{h}$ there is none needed to enforce a continuity requirement. Thus $A$ (and hence $A^{-1}$ ) is block diagonal, and the structure of the linear system (17.5) is the same as that associated with the conforming problem (2.21).

For the dual mixed method the situation is different. Thus while we can not give here an exhaustive treatment of the solution of linear systems of the form (17.2), we would like to mention briefly some techniques that have been used when the system (17.2) results from a dual mixed method.

Remark 17.1 The algebraic system associated with the primal or dual hybrid methods discussed in the following chapter can, under restrictions similar to those indicated above, take the form (17.2). However, as with the primal mixed method, the form of $A$ is such that the linear system that must be solved is that of a more standard problem, again as for a conforming problem for the dual hydrid method, as for a nonconforming problem for the primal hydrid method. Thus no section analogous to this one will be given in Chapter V. 
Conjugate Gratient Method. Even for the dual mixed method where $A^{-1}$ is full, the system (17.5) can be solved using conjugate gradient iteration as $A^{-1}$ never actually has to be computed. Whenever $A^{-1} \psi$ is needed one can solve the system $\mathbf{A} \psi=\psi$ using, for example, Gaussian elimination as $\mathbf{A}$ itself is sparse. However, conjugate gradient iteration without preconditioning is known to converge slowly, and in general there seems to be no obvious preconditioner, though in BROWN (1982) and in EWING \& WHEELER (1983) several preconditioners are proposed and tested for the case of a triangulation by rectangles. Moreover, conjugate gradient iteration is known to be especially sensitive to round-off error.

It seems appropriate to mention that there has been much research concerning the use of the conjugate gradient method and its variants to solve matrix. equations involving matrices that are not necessarily symmetric or positive definite. Thus one may consider applying such a method to solve equation (17.2) even though $\mathbf{L}$ is not positive definite. In JOLY (1984) several variants of the conjugate gradient method are described and their performance in solving equations of the form (17.2) coming from the implementation of a dual mixed method are compared. As pointed out in JOLY (1984), for such systems the role of the preconditioner is no longer simply to speed up the convergence but in fact to guarantee the convergence.

Penalty Method. This method consists of replacing (17.1) by a more regular problem : find $\left(\phi_{h, \epsilon}, \lambda_{h, \epsilon}\right)$ satisfying

$$
\begin{cases}\phi_{h, \epsilon} \in \mathrm{W}_{\mathrm{h}}, & \\ \mathrm{a}\left(\phi_{\mathrm{h}, \epsilon}, \psi_{\mathrm{h}}\right)+\mathrm{b}\left(\psi_{\mathrm{h}} \lambda_{\mathrm{h}, \epsilon}\right)=\left(\mathrm{f}, \psi_{\mathrm{h}}\right), & \text { for all } \psi_{\mathrm{h}} \in \mathrm{W}_{\mathrm{h}} ; \\ \mathrm{b}\left(\phi_{\mathrm{h}, \epsilon}, \mu_{\mathrm{h}}\right)-\epsilon\left(\lambda_{\mathrm{h}, \epsilon}, \mu_{\mathrm{h}}\right)=\left(\mathrm{g}, \mu_{\mathrm{h}}\right), & \text { for all } \mu_{\mathrm{h}} \in \mathrm{M}_{\mathrm{h}}\end{cases}
$$

The corresponding algebraic problem then becomes

$$
\left(\begin{array}{cc}
\mathbf{A} & \mathbf{B}^{*} \\
\mathbf{B} & -\epsilon \mathbf{I}
\end{array}\right)\left(\begin{array}{c}
\phi_{\mathrm{h}, \epsilon} \\
\lambda_{\mathrm{h}, \epsilon}
\end{array}\right)=\left(\begin{array}{c}
f_{\mathrm{h}} \\
g_{\mathrm{h}}
\end{array}\right) \text {, }
$$


(17.8)

$$
\lambda_{h, \epsilon}=\frac{1}{\epsilon}\left(B \phi_{h, \epsilon}-g_{h}\right) \text {. }
$$

to obtain

$$
\left(A+\frac{1}{\epsilon} B^{*} B\right) \phi_{h, \epsilon}=f_{h}+\frac{1}{\epsilon} B^{*} g_{h} \text {. }
$$

For our model problem the matrix $\left(\mathbf{A}+\frac{1}{\epsilon} \mathbf{B}^{*} \mathbf{B}\right)$ is symmetric, positive definite, and sparse, hence (17.9) can be solved by known techniques. In BERCOVIER (1978, Theorem 3.1) such penalized problems are studied and it is shown that, under the more general hypotheses that i) and ii) of Theorem 10.2 and

$$
\inf _{\left\{\psi_{h} \in W_{h} ;\left\|\psi_{h}\right\|_{W_{h}}=1\right\}}\left\{\left|a\left(\psi_{h}, \psi_{h}\right)\right|+\sup _{\left\{\mu_{h} \in M_{h} ;\left\|\mu_{h}\right\|_{M_{h}}=1\right\}} b\left(\psi_{h}, \mu_{h}\right)\right\}>0
$$

hold, there is a unique solution $\left(\phi_{h, \epsilon}, \lambda_{h, \epsilon}\right)$ of (17.6), and if $\left(\phi_{h}, \lambda_{h}\right)$ is the solution of (17.1) then there is a positive constant $C$, independent of $\epsilon$, such that

$$
\left\|\phi_{h^{-}}-\phi_{h, \epsilon}\right\|_{W_{h}}+\left\|\lambda_{h}-\lambda_{h, \epsilon}\right\|_{M_{h}} \leq C \epsilon .
$$

The choice of $\epsilon$ may thus be made independently of $h$; though, as pointed out in BERCOVIER (1978), for each $h$ there is an optimal $\epsilon$.

This method is surely the simplest to implement, but it has one crucial flaw. The equation

$$
\mathbf{B} \phi_{h}=\mathrm{g}_{\mathrm{h}}
$$

is not satisfied by the solution $\phi_{h, \epsilon}$. Thus the method, at least for our model problem, is mostly of historical interest. 
Augmented Lagrangian Method . Under the hypothesis that $a(\cdot, \cdot)$ is symmetric and $W_{h}$-elliptic, $\left(\phi_{h}, \lambda_{h}\right)$ is a solution of $(17.1)$ if and only if $\left(\phi_{h}, \lambda_{h}\right)$ is a saddle point of the Lagrangian $\Gamma$ defined on $W_{h} \times M_{h}$ as follows :

$$
\Gamma\left(\psi_{h}, \mu_{h}\right)=\frac{1}{2} a\left(\psi_{h}, \psi_{h}\right)+b\left(\psi_{h}, \mu_{h}\right)-f\left(\psi_{h}\right)-g\left(\mu_{h}\right)
$$

The classical iterative method of Uzawa to obtain the saddle point of $\Gamma$ is described by the algorithm :

Intialize :

$$
\text { Let } \lambda_{h}^{(0)} \text { be an arbitrarily chosen element of } M_{h} \text {. }
$$

Calculate $\phi_{\mathrm{h}}$ :

$$
\begin{aligned}
& \text { Once } \lambda_{h}^{(n)} \text { is known, } \phi_{h}^{(n)} \text { is defined to be the element of } w_{h} \text { minimizing } \\
& \Gamma\left(\psi_{h}, \lambda_{h}^{(n)}\right) \text {; i.e. } \phi_{h}^{(n)} \text { is the solution of } \\
& \quad A \phi_{h}^{(n)}=f_{h}-B^{*} \lambda_{h}^{(n)} .
\end{aligned}
$$

Advance $\lambda_{\mathrm{h}}$ :

$$
\text { Let } \lambda_{h}^{(n+1)} \text { be defined by }
$$

$$
\lambda_{h}^{(n+1)}=\lambda_{h}^{(n)}+\rho_{n}\left(B \phi_{h}^{(n)}-g_{h}\right) .
$$

This method in general converges quite slowly. The idea of the augmented Lagrangian method is to "penalize" the Lagrangian $\Gamma$ to obtain a Lagrangian $\Gamma_{r}$ for which Uzawa's method converges more rapidy. For $r>0$, put

$$
\Gamma_{r}\left(\psi_{h}, \mu_{h}\right)=\Gamma\left(\psi_{h}, \mu_{h}\right)+\frac{r}{2}\left\|B \psi_{h}-g_{h}\right\|^{2}
$$


It is easy to see that $\left(\phi_{h}, \lambda_{h}\right)$ is a saddle point of $\Gamma_{r}$ if and only if it is a saddle point of $\Gamma$. Thus this method is not really a penalty method as $\Gamma$ does not have to tend toward $\infty$ to obtain the actual solution, and the augmented Lagrangian method does not have the defect of the earlier described penalty method. Applying Uzawa's method to $r_{r}$ one obtains the algorithm :

Initialize :

Let $\lambda_{h}^{(0)}$ be an arbitrarily chosen element of $M_{h}$.

Calculate $\phi_{\mathrm{h}}$ :

Once $\lambda_{h}^{(n)}$ is known, $\phi_{h}^{(n)}$ is defined to be the element of $w_{h}$ minimizing

$\Gamma_{r}\left(\psi_{h}, \lambda_{h}^{(n)}\right) ;$ i.e. $\phi_{h}^{(n)}$ is the solution of

$$
A_{r} \phi_{h}^{(n)}=\left(A+r B^{*} B\right) \phi_{h}^{(n)}=f_{h}-B^{*} \lambda_{h}^{(n)}+r B^{*} g_{h}
$$

Advance $\lambda_{\mathrm{h}}$ :

$$
\text { Let } \lambda_{h}^{(n+1)} \text { be defined by }
$$

$$
\lambda_{h}^{(n+1)}=\lambda_{h}^{(n)}+\rho_{n}\left(B \phi_{h}^{(n)}-g_{h}\right)
$$

The augmented Lagrangian method orginated with the work of HESTENES (1969) and POWELL (1969) and is studied extensively in FORTIN \& GLOWINSKI (1983) where the following two results are shown (theorems 2.1 and 2.2) under the hypothesis that $\mathbf{A}$ is symmetric and positive definite:

i) There is a positive constant $\alpha$ such that if $\alpha \leq \rho_{n} \leq 2 r$ for each $n$, then the sequence $\phi_{h}^{(n)}$ converges to the solution $\phi_{h}$.

ii) If $\hat{\lambda}_{h}$ is the unique element of Im B satisfying (17.1) with $\phi_{h}$, then the sequence $\lambda_{h}^{(n)}$ converges to $\lambda_{h}+\lambda^{\circ}$, where $\lambda^{\circ}$ is the componant of $\lambda_{h}^{(0)}$ in $\operatorname{Ker} \mathbf{B}^{*}$. 
Remark 17.2 . The algorithm of Uzawa, and hence that of the augmented Lagrangian, can be interpreted in the following manner: replace problem (17.1) by the virtual time dependent problem

$$
\begin{cases}a\left(\phi_{h}(t), \psi_{h}\right)+b\left(\psi_{h}, \lambda_{h}(t)\right)=\left(f, \psi_{h}\right) & \text { for all } \psi_{h} \in W_{h} \\ b\left(\phi_{h}(t), \mu_{h}\right)-\left(\frac{d}{d t} \lambda_{h}(t), \mu_{h}\right)=\left(g, \mu_{h}\right) & \text { for all } \mu_{h} \in M_{h}\end{cases}
$$

and look for the solution $\left(\phi_{h}, \lambda_{h}\right)$ as the steady state solution of (17.18). Introducing an explicit time discretization with $n^{\text {th }}$ time step $\rho_{n}=t^{n+1}-t^{n}$, one obtains

$$
\begin{cases}a\left(\phi_{h}^{(n)}, \psi_{h}\right)+b\left(\psi_{h}, \lambda_{h}^{(n)}\right)=\left(f, \psi_{h}\right) & \text { for all } \psi_{h} \in W_{h}, \\ b\left(\phi_{h}^{(n)}, \mu_{h}\right)-\left(\frac{\lambda_{h}^{(n+1)}-\lambda_{h}^{(n)}}{\rho_{n}}, \mu_{h}\right)=\left(g, \mu_{h}\right) & \text { for all } \mu_{h} \in M_{h},\end{cases}
$$

which gives the algebraic system

$$
\left\{\begin{array}{l}
\mathbf{A} \phi_{h}^{(n)}+\mathbf{B}^{*} \lambda_{h}^{(n)}=f_{h} \\
\rho_{n} B \phi_{h}^{(n)}-\left(\lambda_{h}^{(n+1)}-\lambda_{h}^{(n)}\right)=\rho_{n} g_{h}
\end{array}\right.
$$

for Uzawa's method and

$$
\left\{\begin{array}{l}
\left(A+r B^{*} B\right) \phi_{h}^{(n)}+B^{*} \lambda_{h}^{(n)}=f_{h}+r B^{*} g_{h} \\
\rho_{n} B \phi_{h}^{(n)}-\left(\lambda_{h}^{(n+1)}-\lambda_{h}^{(n)}\right)=\rho_{n} g_{h}
\end{array}\right.
$$

for the augmented Lagrangian method. 
Remark 17.3 . Uzawa's algorithm can be viewed as a gradient type algorithm for the minimization of the functional $J_{*}$ on $M_{h}$ :

$$
\begin{aligned}
J_{*}\left(\mu_{h}\right) & =-\min _{\psi_{h} \in W_{h}} \Gamma\left(\psi_{h}, \mu_{h}\right) \\
& =\frac{1}{2}\left(B^{-1} B^{*} \mu_{h}, \mu_{h}\right)-\left(B^{-1} f_{h}, \mu_{h}\right)+\frac{1}{2}\left(A^{-1} f_{h}, f_{h}\right)+\left(g_{h}, \mu_{h}\right),
\end{aligned}
$$

in that eliminating $\phi_{\mathrm{h}}^{(\mathrm{n})}$ in (17.13) and (17.14),

$$
\phi_{h}^{(n)}=A^{-1}\left(f_{h}-B^{*} \lambda_{h}^{(n)}\right)
$$

we obtain the algorithm

Initialize :

$$
\text { Let } \lambda_{h}^{(0)} \text { be an arbitrarily chosen element of } M_{h} \text {. }
$$

Advance $\lambda_{\mathrm{h}}$ :

$$
\begin{aligned}
& \text { Once } \lambda_{h}^{(n)} \text { is known, put } \\
& \lambda_{h}^{(n+1)}=\lambda_{h}^{(n)}-\rho_{n}\left(B A^{-1} B^{*} \lambda_{h}^{(n)}-B^{-1} f_{h}+g_{h}\right) .
\end{aligned}
$$

Thus the augmented Lagrangian algorithm can be viewed as a gradient type algorithm for the minimization of the dual functional $J_{*_{r}}$ on $M_{h}$ :

$$
\begin{gathered}
J_{*_{r}}\left(\mu_{h}\right)=-\min _{\psi_{h} \in W_{h}} \Gamma_{r}\left(\psi_{h}, \mu_{h}\right) \\
=\frac{1}{2}\left(B A_{r}^{-1} B^{*} \mu_{h}, \mu_{h}\right)-\left(B A_{r}^{-1}\left(f_{h}+r B^{*} g_{h}\right), \mu_{h}\right)+\frac{1}{2}\left(A_{r}^{-1}\left(f_{h}+r B^{*} g_{h}\right), f_{h}+r B^{*} g_{h}\right) .
\end{gathered}
$$

However $A_{r}^{-1}$, like $A^{-1}$, is, a priori, full. Similarly, one may eliminate $\lambda_{h}^{(n)}$ to obtain an algortihm involving $\phi_{h}^{(n)}$, but again the matrix $A_{r}^{-1}$ appears. 
Uzawa's algorithm indicates no specific method for solving the equation determining $\phi_{h}^{(n)}$. As $A$ and $A_{r}$ are positive definite and sparse, either a direct method such as Gaussian elimination or an iterative method may be used to determine $\phi_{\mathrm{h}}^{(\mathrm{n})}$ in (17.13) or (17.16). The Arrow-Hurwitz algorithm is an algorithm that may be interpreted as being a variant of the Uzawa algorithm in which the method for calculating $\phi_{h}^{(n)}$ is specified :

\section{Initialize :}

Let $\lambda_{h}^{(0)}$ and $\phi_{h}^{(0)}$ be arbitrarily chosen elements of $M_{h}$ and $W_{h}$, respectively.

Advance $\phi_{h}$ :

$$
\begin{gathered}
\text { Once } \lambda_{h}^{(n)} \text { and } \phi_{h}^{(n)} \text { are known, } \phi_{h}^{(n+1)} \text { is defined by } \\
\phi_{h}^{(n+1)}=\phi_{h}^{(n)}-\omega_{n}\left(A \phi_{h}^{(n)}+B^{*} \lambda_{h}^{(n)}-f_{h}\right) .
\end{gathered}
$$

Advance $\lambda_{h}$ :

$$
\begin{aligned}
& \text { Let } \lambda_{h}^{(n+1)} \text { be defined by } \\
& \lambda_{h}^{(n+1)}=\lambda_{h}^{(n)}+\rho_{n}\left(B \phi_{h}^{(n)} g_{h}\right) .
\end{aligned}
$$

Thus a variant of the augmented Lagrangian method is obtained by replacing $A$ by $A_{r}$ in (17.19).

Remark 17.4 : If $\omega_{n}$ is taken to be equal to $\hat{p}_{n}$, then the algorithm of Arrow-Hurwitz is also associated with the virtual time dependent problem

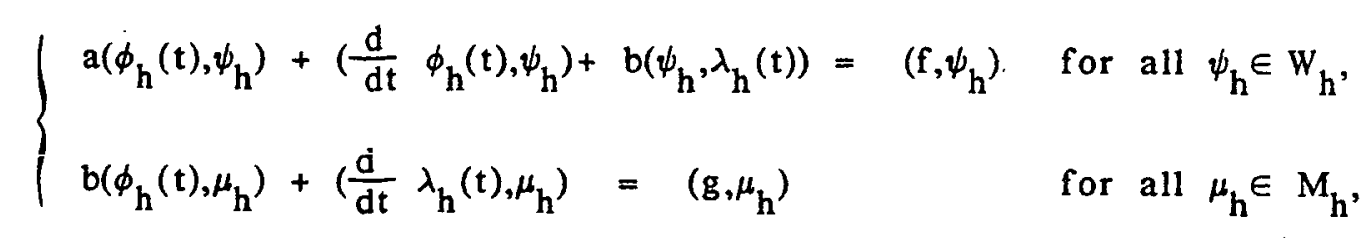

of which the solution of $(17.1)$ is the steady state solution. 
Alternating-Direction Method. These methods can be used both in 2 dimensions and in 3 dimensions when the triangulation $T_{h}$ of the domain $\Omega$ is a triangulation by rectangles. Here again one introduces a virtual time dependent problem to obtain the solution of the original problem as its steady state. The idea is, heuristically speaking, to reduce the solution of the two(or three-) dimensional problem to the solution of one-dimensional problems by advancing half (or a third of) a time step in the $x_{1}$-direction, then half (or a third of) a time step in the $x_{2}$-direction (and in the three-dimensional case, a third of a time step in the $x_{3}$-direction).

For example, in the two-dimensional case, for the Raviart-Thomas elements the subspace $W_{h}$ of $H(\operatorname{div} ; \Omega)$ in which the vectors are approximated is locally $P_{k+1, k} \times P_{k, k+1}$ and we suppose that the basis $\left\{w_{j}\right\}_{j=1}^{2 m}$ has been chosen such that $w_{h, 1}=\operatorname{span}\left(w_{j}\right\}_{j=1}^{m}$ is locally $\dot{P}_{k+1, k} \times\{0\}$, and $w_{h, 2}=$ $\operatorname{span}\left\{w_{j}\right\}_{j=m+1}^{2 m}$ is locally $\{0\} \times P_{k, k+1}$. Thus the vector $w_{h} \in w_{h}$ may be written uniquely as $w_{h}=w_{h, 1}+w_{h, 2}$ where $w_{h, 1} \in w_{h, 1}$ and $w_{h, 2} \in w_{h, 2}$, and the matrices $\mathbf{A}$ and $\mathbf{B}$ are block diagonal :

$$
\mathbf{A}=\left(\begin{array}{ll}
\mathbf{A}_{1} & 0 \\
0 & \mathbf{A}_{2}
\end{array}\right), \quad \mathbf{B}=\left(\begin{array}{ll}
\mathbf{B}_{1} & 0 \\
0 & \mathbf{B}_{2}
\end{array}\right) \text {. }
$$

As in Remark 17.4 a fictitious time dependent problem is introduced :

$$
\begin{cases}a\left(\phi_{h}(t), \psi_{h}\right)+b\left(\psi_{h}, \lambda_{h}(t)\right)=\left(f, \psi_{h}\right) & \text { for all } \psi_{h} \in W_{h}, \\ b\left(\phi_{h}(t), \mu_{h}\right)-\left(\frac{d}{d t} \lambda_{h}(t), \mu_{h}\right)=\left(g, \mu_{h}\right) & \text { for all } \mu_{h} \in M_{h} .\end{cases}
$$

The two step iterative procedure is obtained by introducing an implicit time discretization as follows : 


$$
\begin{aligned}
& \begin{cases}\mathrm{a}\left(\phi_{\mathrm{h}, 1}^{\left(\mathrm{n}^{*}\right)}+\phi_{\mathrm{h}, 2}^{(\mathrm{n})}, \psi_{\mathrm{h}}\right)+\mathrm{b}\left(\psi_{\mathrm{h}}, \lambda_{\mathrm{h}}^{\left(\mathrm{n}+\frac{1}{2}\right)}\right)=\left(\mathrm{f}, \psi_{\mathrm{h}}\right) & \text { for all } \psi_{\mathrm{h}} \in \mathrm{w}_{\mathrm{h}, 1}, \\
\mathrm{~b}\left(\phi_{\mathrm{h}, 1}^{\left(\mathrm{n}^{*}\right)}+\phi_{\mathrm{h}, 2}^{(\mathrm{n})}, \mu_{\mathrm{h}}\right)-\left(\frac{\lambda_{\mathrm{h}}^{\left(\mathrm{n}+\frac{1}{2}\right)}-\lambda_{h}^{(\mathrm{n})}}{\rho_{\mathrm{n}}}, \mu_{h}\right)=\left(\mathrm{g}, \mu_{\mathrm{h}}\right) & \text { for all } \mu_{\mathrm{h}} \in \mathrm{M}_{\mathrm{h}},\end{cases} \\
& \begin{cases}a\left(\phi_{h, 1}^{\left(n^{*}\right)}+\phi_{h, 2}^{(n+1)}, \psi_{h}\right)+b\left(\psi_{h}, \lambda_{h}^{(n+1)}\right)=\left(f, \psi_{h}\right) & \text { for all } \psi_{h} \in W_{h, 2}, \\
b\left(\phi_{h, 1}^{\left(n^{*}\right)}+\phi_{h, 2}^{(n+1)}, \mu_{h}\right)-\left(\frac{\lambda_{h}^{(n+1)}-\lambda_{h}^{\left(n+\frac{1}{2}\right)}}{\rho_{n}}, \mu_{h}\right)=\left(g, \mu_{h}\right) & \text { for all } \mu_{h} \in M_{h},\end{cases}
\end{aligned}
$$

which gives the algebraic systems

$$
\left\{\begin{array}{l}
A_{1} \phi_{h, 1}^{\left(n^{*}\right)}+B_{1}^{*} \lambda_{h}^{\left(n+\frac{1}{2}\right)}=f_{h, 1}-A_{2} \phi_{h, 2}^{(n)} \\
\rho_{n} B_{1} \phi_{h, 1}^{\left(n^{*}\right)}-\lambda_{h}^{\left(n+\frac{1}{2}\right)}=\rho_{n}\left(g_{h}-B_{2} \phi_{h, 2}^{(n)}\right)-\lambda_{h}^{(n)}
\end{array}\right.
$$

and

$$
\left\{\begin{array}{l}
\mathbf{A}_{2} \phi_{h}^{(n+1)}+\mathbf{B}_{2}^{*} \lambda_{h}^{(\mathrm{n}+1)}=\mathbf{f}_{h, 2}-\mathbf{A}_{1} \phi_{h, 1}^{\left(\mathrm{n}^{*}\right)} \\
\rho_{\mathrm{n}} \mathbf{B}_{2} \phi_{h}^{(\mathrm{n}+1)}-\lambda_{h}^{(\mathrm{n}+1)}=\rho_{\mathrm{n}}\left(\mathrm{g}_{\mathrm{h}}-\mathbf{B}_{1} \phi_{\mathrm{h}, 1}^{\left(\mathrm{n}^{*}\right)}\right)-\lambda_{h}^{\left(\mathrm{n}+\frac{1}{2}\right)}
\end{array}\right.
$$

From the first pair of the above equations $\lambda_{h}^{\left(n+\frac{1}{2}\right)}$ can be eliminated to obtain

$$
\left(\mathbf{A}_{1}+\rho_{\mathrm{n}} \mathbf{B}_{1}^{*} \mathbf{B}_{1}\right) \phi_{\mathrm{h}, 1}^{\left(\mathrm{n}^{*}\right)}=\mathrm{f}_{\mathrm{h}, 1}-\rho_{\mathrm{n}} \mathbf{B}_{1}^{*} \mathrm{~g}_{\mathrm{h}}-\left(\mathbf{A}_{2}+\rho_{\mathrm{n}} \mathbf{B}_{1}^{*} \mathbf{B}_{2}\right) \phi_{\mathrm{h}, 2}^{(\mathrm{n})}+\mathbf{B}_{1}^{*} \lambda_{\mathrm{h}}^{(\mathrm{n})},
$$

and from the second pair, eliminating $\lambda_{h}^{(n+1)}$, one obtains

$$
\left(\mathbf{A}_{2}=\rho_{\mathbf{n}} \mathbf{B}_{2}^{*} \mathbf{B}_{2}\right) \phi_{h, 2}^{(n+1)}=f_{h, 2}-\rho_{n} \mathbf{B}_{2}^{*} g_{h}-\left(A_{1}+\rho_{n} \mathbf{B}_{2}^{*} \mathbf{B}_{1}\right) \phi_{h, 1}^{\left(n^{*}\right)}+\mathbf{B}_{2}^{*} \lambda_{h}^{\left(n+\frac{1}{2}\right)} .
$$

Thus the algorithm may be given as follows : 
Initialize :

Let $\lambda_{h}^{(0)}$ be an arbitrarily chosen element of $M_{h}$.

The initialization $\phi_{\mathrm{h}, 2}^{(0)}$ of $\phi_{\mathrm{h}, 2}$ is obtained as the solution of

$$
A_{2} \phi_{h, 2}^{(0)}=f_{h, 2}-B_{2}^{*} \lambda_{h}^{(0)}
$$

Advance a half time step in the $\mathrm{x}_{1}$ direction :

$$
\text { Once } \lambda_{h}^{(n)} \text { and } \phi_{h, 2}^{(n)} \text { are known, }
$$

$$
\left(A_{1}+\rho_{n} B_{1}^{*} B_{1}\right) \phi_{h, 1}^{\left(n^{*}\right)}=f_{h, 1}-\rho_{n} B_{1}^{*} g_{h}-\left(A_{2}+\rho_{n} B_{1}^{*} B_{2}\right) \phi_{h, 2}^{(n)}+B_{1}^{*} \lambda_{h}^{(n)} .
$$

Let $\lambda_{h}^{\left(n+\frac{1}{2}\right)}$ be defined by

$$
\lambda_{h}^{\left(n+\frac{1}{2}\right)}=\lambda_{h}^{(n)}+\rho_{n}\left(B_{1} \phi_{h, 1}^{\left(n^{*}\right)}+B_{2} \phi_{h, 2}^{(n)}-g_{h}\right)
$$

Advance a half time step in the $\mathrm{x}_{2}$ direction:

Once $\lambda_{h}^{\left(n+\frac{1}{2}\right)}$ and $\phi_{h, 1}^{\left(n^{*}\right)}$ are known,

$$
\left(\mathbf{A}_{2}+\rho_{n} \mathbf{B}_{2}^{*} \mathbf{B}_{2}\right) \phi_{h, 2}^{(\mathrm{n}+1)}=\mathrm{f}_{\mathrm{h}, 2}-\rho_{\mathrm{n}} \mathbf{B}_{2}^{*} \mathrm{~g}_{\mathrm{h}}-\left(\mathbf{A}_{1}+\rho_{\mathrm{n}} \mathbf{B}_{2}^{*} \mathbf{B}_{1}\right) \phi_{\mathrm{h}, 1}^{\left(\mathrm{n}^{*}\right)}+\mathbf{B}_{2}^{*} \lambda_{\mathrm{h}}^{\left(\mathrm{n}+\frac{1}{2}\right)}
$$

Let $\lambda_{h}^{(n+1)}$ be defined by

$$
\lambda_{h}^{(n+1)}=\lambda_{h}^{\left(n+\frac{1}{2}\right)}+\rho_{n}\left(B_{1} \phi_{h, 1}^{\left(n^{*}\right)}+B_{2} \phi_{h, 2}^{(n+1)}-g_{h}\right) .
$$

Correct :

Finally to obtain $\phi_{\mathrm{h}, 1}^{(\mathrm{n}+1)}$, solve

$$
A_{1} \phi_{h, 1}^{(n+1)}=f_{h, 1}-B_{1}^{*} \lambda_{h}^{(n+1)}
$$


This proceedure without the correction step was introduced by BROWN (1982), and as given here by DOUGLAS, DURAN \& PIETRA (1987a), (1987b). It is an adaptation of the original alternating-direction procedure of PEACEMAN \& RACHFORD (1955). Also in DOUGLAS, DURAN \& PIETRA (1987a), (1987b) an alternating-direction scheme for the three-dimensional case is introduced. This procedure is based on the scheme of DOUGLAS (1962). As with the classical alternating directions itrerative schemes, only limited convergence results have been obtained; cf. the above references.

For the Brezzi-Douglas-Marini elements there is no natural decomposition of $W_{h}$ as for the Raviart-Thomas elements. Thus the above algorithm is not applicable. However, an alternating-direction procedure for these elements involving the introduction of two virtual time variables is given for 2 dimensions in DOUGLAS \& PIETRA (1986) and for 3 dimensions in DOUGLAS, DURAN \& PIETRA (1987a), (1987b).

Mixed Hybrid Method . As pointed out earlier, the reason that the algebraic system derived from the dual mixed method poses special problems is that due to the degrees of freedom imposing the interelement continuity requirements on the elements $\psi_{h}$ of $W_{h}$, the matrix $A^{-1}$ is likely to be full. A technique often used to overcome this problem is to replace the dual mixed formulation by a mixed hybrid formulation,cf. FRAEIJS DE VEUBEKE (1965). The idea is to eliminate the interelement continuity requirements from the space $W_{h}$ obtaining a space $\hat{W}_{h}$ and to impose instead the desired continuity on the solution $\hat{\phi}_{h} \in$ $\hat{\mathrm{W}}_{h}$ via Lagrangian multipliers. Problem (17.1) is thus replaced by a problem of the following form : find $\hat{\phi}_{h}, \hat{\lambda}_{h}$, and $\eta_{h}$ satisfying

$$
\left\{\begin{array}{l}
\hat{\phi}_{h} \epsilon \hat{w}_{h}, \quad \hat{\lambda}_{h} \epsilon M_{h}, \quad \eta_{h} \in N_{h}, \\
a\left(\hat{\phi}_{h}, \psi_{h}\right)+b\left(\psi_{h}, \hat{\lambda}_{h}\right)+c\left(\psi_{h}, \eta_{h}\right) \\
b\left(\hat{\phi}_{h}, \mu_{h}\right) \\
c\left(\hat{\phi}_{h}, \tau_{h}\right)
\end{array}\right.
$$

$$
\begin{array}{ll}
=\left(f, \psi_{h}\right) & \text { for all } \psi_{h} \in \hat{W}_{h}, \\
=\left(g, \mu_{h}\right) & \text { for all } \mu_{h} \in M_{h}, \\
=0 & \text { for all } \tau_{h} \in N_{h},
\end{array}
$$


where $N_{h} \underset{T^{\prime}<T \in T_{h}}{\subset} L^{2}\left(T^{\prime}\right)$ is the space of Lagrangian multipliers, and the bilinear form $c(\cdot, \cdot)$ on. $\hat{W}_{h} \times N_{h}$ is such that the induced linear map $C$ from $W_{h}$ into $N_{h}$ has as kernel the elements of $\hat{W}_{h}$ which belong in fact to $W_{h}$. The linear system thus obtained is

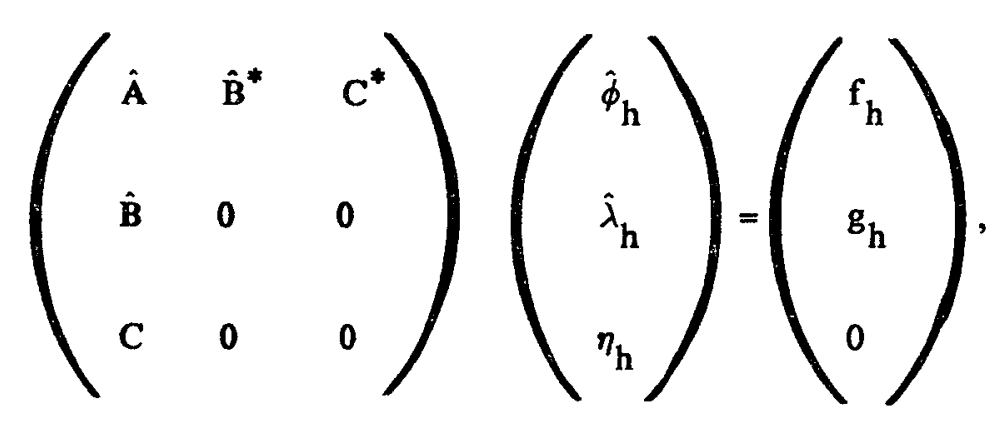

where $\hat{\mathbf{A}}$ is now block diagonal as well as symmetric and positive definite, and $\hat{\phi}_{h}$ and $\hat{\lambda}_{h}$ are equal as functions to $\phi_{h}$ and $\lambda_{h}$, respectively. Eliminating $\hat{\phi}_{h}$ from the system,

$$
\hat{\phi}_{h}=\hat{\mathbf{A}}^{-1}\left(\mathrm{f}_{\mathrm{h}}-\hat{\mathbf{B}}^{*} \hat{\lambda}_{\mathrm{h}}-\mathrm{C}^{*} \eta_{\mathrm{h}}\right)
$$

we obtain

$$
\left(\begin{array}{cc}
\hat{\mathbf{B}} \hat{\mathbf{A}}^{-1} \hat{\mathbf{B}}^{*} & \hat{\mathbf{B}} \hat{\mathbf{A}}^{-1} \mathrm{C}^{*} \\
\mathrm{C} \hat{\mathbf{A}}^{-1} \hat{\mathbf{B}}^{*} & \mathrm{C} \hat{\mathbf{A}}^{-1} \mathrm{C}^{*}
\end{array}\right)\left(\begin{array}{l}
\hat{\lambda}_{\mathrm{h}} \\
\eta_{\mathrm{h}}
\end{array}\right)=\left(\begin{array}{c}
\hat{\mathbf{B}} \hat{\mathbf{A}}^{-1} \mathrm{f}_{\mathrm{h}^{-}} \mathrm{g}_{\mathrm{h}} \\
\mathrm{C} \hat{\mathbf{A}}^{-1} \mathrm{f}_{\mathrm{h}}
\end{array}\right),
$$

and the space $N_{h}$ and the form $c(\bullet, \cdot)$ have been constructed in such a way that this system is symmetric and positive definite. But now we further note that $\hat{\mathbf{B}} \hat{\mathbf{A}}^{-1} \mathbf{B}^{*}$ is also block diagonal. Thus we have

$$
\hat{\lambda}_{h}=\left(\hat{\mathbf{B}} \hat{A}^{-1} \hat{\mathbf{B}}^{*}\right)^{-1}\left\{\hat{\mathbf{B}} \hat{A}^{-1} \mathrm{f}_{\mathrm{h}}-\mathrm{g}_{\mathrm{h}}-\hat{\mathbf{B}} \hat{\mathbf{A}}^{-1} \mathbf{C}^{*} \eta_{\mathrm{h}}\right\}
$$

and

$$
\begin{aligned}
\left\{C \hat{A}^{-1} C^{*}-C \hat{A}^{-1} \hat{\mathbf{B}}^{*}\left(\hat{\mathbf{B}} \hat{A}^{-1} \hat{\mathbf{B}}^{*}\right)^{-1} \hat{\mathbf{B}} \hat{A}^{-1} \mathbf{C}^{*}\right\} \eta_{h} \\
=\left\{C \hat{\mathbf{A}}^{-1}-C \hat{\mathbf{A}}^{-1} \hat{\mathbf{B}}^{*}\left(\hat{\mathbf{B}} \hat{\mathbf{A}}^{-1} \hat{\mathbf{B}}^{*}\right)^{-1} \hat{\mathbf{B}} \hat{A}^{-1}\right\} \mathrm{f}_{\mathrm{h}} \\
+C \hat{\mathbf{A}}^{-1} \mathbf{B}^{*}\left(\mathbf{B} \hat{\mathbf{A}}^{-1} \mathbf{B}^{*}\right)^{-1} \mathrm{~g}_{\mathrm{h}} .
\end{aligned}
$$


This system is symmetric, positive definite, and sparse. Thus we can obtain $\eta_{h}$ by solving (17.32), and then $\hat{\lambda}_{h}$ and $\hat{\phi}_{h}$ are given by (17.31) and (17.30), respectively. It is interesting to note that $\eta_{h}$ often has a physical significance. For our model problem $\eta_{h}$ represents the value of $\phi_{h}$ on the faces $T^{\prime}$ and in fact in certain cases can be used together with $\hat{\phi}_{h}$ to obtain a new approximation that converges more rapidly than $\hat{\phi}_{h}$ or equivalently $\phi_{h}$; cf. ARNOLD \& BREZZI (1985) and BREZZI, DOUGLAS \& MARINI (1985). Mixed hybrid methods will be taken up again in Section 21 . 
stress elements, Comput. Methods Appl. Mech. Engrg. $47,331-356$.

QUARTERONI, A. (1979) : Error estimates for the assumed stresses hybrid methods in the approximation of fourth order elliptic equations, R.A.I.R.O. Anal. Numér. 13,355-367.

QUARTERONI, A. (1980a) : On mixed methods for fourth order problems, Comput. Methods Appl. Mech. Engrg. $24,13-24$.

QUARTERONI, A. (1980b) : Mixed approximations of evolution problems, Comput. Methods Appl. Mech. Engrg. $24,137-163$.

RABIER, P. ; THOMAS, J.-M. (1985) : "Introduction a I'Analyse Numerique des Equations aux Derivees Partielles, Exercices". Masson, Paris.

RANNACHER, R. (1979) : On nonconforming and mixed finite element methods for plate bending problems. The linear case, R.A.I.R.O. Anal. Numèr. $13,369-387$.

RAVIART, P.-A. (1984) : Mixed finite element methods, in "The Mathematical Basis of Finite Element Methods, with Applications to Partial Differential Equations "( D.F.Griffiths, Ed.) . pp. 123-156, Clarendon Press, Oxford.

RAVIART, P.-A. ; THOMAS, J.-M. (1977a) : A mixed finite element method for second order elliptic problems, in "Mathematical Aspects of Finite Element Methods" (1. Galligani \& E. Magenes, Eds.), pp.292-315, Lecture Notes in Mothematics 606 . Springer-Verlag. Berlin.

RAVIART, P.-A. ; THOMAS, J.-M. (1977b) : Primal hybrid finite element methods for second order elliptic equations. Math. Comput. $31,391-413$.

RAVIART, P.-A. ; THOMAS, J.-M. (1979) : Dual finite element models for second order elliptic problems, in "Energy Methods in Finite Element Analysis "( R. Glowinski, E.Y. Rodin, \& O.C. Zienkiewicz, Eds.), pp.175-191, John Wiley \& Sons, Chichester.

RAVIART, P.-A. : THOMAS, J.-M. (1983): "Introduction à l'Analyse Numérique des Equations aux Dérivees Partielles, Cours". Masson, Paris.

REDOY, J.N. ; ODEN, J.T. (1973) : Convergence of mixed finite-element approximations of a class of linear boundary-value problems, J. Struct. Mech. 2, 83-108.

REDDY, J.N. ; ODEN, J.T. (1975) : Mixed finite element approximations of linear boundary-value problems, Quart. Appl. Math. 33 , 255-280.

REISSNER, E. (1984): On a certain mixed variational theorem and a proposed application. Internat. J. Numer. Meths. Engrg. 20. 1366-1368.

RUBINSTEIN, R. ; PUNCH, E. ; ATLURI, S.N. (1983) : An analysis of and remedies for, kinematic modes in hybrid stress finite elements: selection of stable, invariant stress fields, Comput. Methods Appl. Mech. Engrg. 38, 63-92. 
SAMUELSSON, A. (1979) : The global constant strain condition and the patch test, in "Energy Methods in Finite Element Analysis" (R. Glowinski, E.Y. Rodin, \& O.C. Zienkiewicz, Eds.), Pp.47-58, John Wiley \& Sons, Chichester.

SANDER, G. ; BECKERS P. (1977) : The influence of the choise of connectors in finite element method, Internat. J. Numer. Meths. Engrg. 11, 1491-1505.

SCAPOLLA, T. (1980) : A mixed finite element method for the biharmonic problem, R.A.I.R.O. Anal. Numer. $14,55-79$.

SCHOLZ, R. (1976) : Approximation von Sattelpunkten mit finiten Elementen, Tagungsband. Math. Schriften $89,53-66$.

SCHOLZ, R. (1977) ; $L$-convergence of saddle-point approximations for second order problems, R.A.I.R.0. Anal. Numer. 11, 209-216.

SCHOLZ, R. (1978) : A mixed method for fourth order problems using linear finite elements, R.A.I.R.O. Anal. Numer. $12,85-90$.

SERRIN J. (1959) : Mathematical Principles of Classical Fluid Mechanics, in "Handdbuch der Physik" (S. Flugge, Ed.), Band VIII/1: Stromungs Mechanik I. (C. Truesdell, Coed.), pp.125-260, Springer Verlog. Berlin.

SHIMODAIRA, H. (1985) : Equivalence between mixed models and displacement models using reduced integration, Internat. J. Numer. Meths. Engrg. $21,89-104$.

SPILKER, R.L. (1982) : Invariant 8-node hybrid-stress ele ments for thin and moderately thick plates, Internat. J. Numer. Meths. Engrg. 18, 1153-1178.

SPILKER, R.L. (1983) : Hybrid-stress reduced Mindlin isoparametric elements for analysis of thin plates. J. Structural Mech. 11 , 49-66.

SPILKER, R.L. ; BELYTSCHKO, T. (1983) : Bilinear Mindlin plate elements, in "Hybrid and Mixed Finite Element Methods " (S.N. Atluri, R.H. Gallogher, \& O.C. Zienkiewicz, Eds.), pp.117-136, John Wiley \& Sons, Chichester.

SPILKER, R.L. ; MASKERI, S.M. ; KANIA, E. (1981) : Plane i soparametric hybrid-stress elements, Internat. J. Numer. Meths. Engrg. 17 , 1469-1496.

SPILKER, R.L. ; MUNIR, N.I. (1980a) : The hybrid-stress model for thin plates. Internat. J. Numer. Meths. Engrg. 15. 1239-1260.

SPILKER, R.L. ; MUNIR, N.I. (1980b) : A hybrid-stress quadratic serendipity displacement Mindlin plate bending element. Comput. \& Structures $12,11-21$.

SPILKER, R.L. ; MUNIR, N.I. (1980c) : A serendipity cubicdisplacement hybrid-stress element for thin and moderately thick plates, Internat. J. Numer. Meths. Engrg. $15,1261-1278$.

STANLEY, G.M. ; PARK, K.C. ; HUGHES, T.J.R. (1986) : Treatement of large rotations of thin shells using mixed finite 


\section{CHAPTER V}

\section{HYBRID FINTTE ELEMENT METHODS}

\section{8 - EXAMPLES OF PRIMAL HYBRID FINITE ELEMENT METHODS}

We shall develop several examples of hybrid formulations for the model problem : find $p$ and $u$ satisfying the system of first order partial differential equations $(4.4)^{\circ}-(4.5)$ and the Robin's boundary condition (15.1): $p \cdot \nu+t u=g$ on $\Gamma$, with $t$ a continuous, strictly positive function on $\Gamma$. We shall indicate how to adapt the method to treat a problem with Dirichlet or Neumann boundary conditions.

To a triangulation $T_{h}$ of the domain $\bar{\Omega}$, we have associated the spaces $\mathrm{H}^{1}\left(T_{\mathrm{h}}\right)$ and $H\left(\operatorname{div} ; T_{\mathrm{h}}\right)$, cf. (5.11) and (6.16). The space $\mathrm{H}^{1}\left(T_{\mathrm{h}}\right)$ is a subspace of $\mathrm{L}^{2}(\Omega)$ isomorphic to the product space $\Pi_{\mathrm{T} \in T_{\mathrm{h}}} \mathrm{H}^{1}(\mathrm{~T})$, and the space $H\left(\mathrm{div} ; T_{\mathrm{h}}\right)$ is a

subspace of $\left(\mathrm{L}^{2}(\Omega)\right)^{\mathrm{n}}$ isomorphic to the product space $\mathrm{\Pi} H(\mathrm{div} ; \mathrm{T})$. The hybrid $T \in T_{h}$

formulations, both primal and dual, will make use of a Lagrangian multiplier belonging to the space $L^{2}\left(\partial T_{h}\right)$ which is defined to be the product space

$$
L^{2}\left(\partial T_{h}\right)=\prod_{T \in T_{h}} L^{2}(\partial T) .
$$

For the primal hybrid formulation, the subspace of multipliers will be

$$
\begin{gathered}
\mathrm{E}\left(\partial T_{\mathrm{h}}\right)=\left\{\mu=\left(\mu_{\mathrm{T}}\right) \mathrm{T} \in T_{\mathrm{h}} \in \mathrm{L}^{2}\left(\partial T_{\mathrm{h}}\right) ; \exists \underline{\sim} \in \mathrm{H}(\operatorname{div} ; \Omega)\right. \\
\text { s.t. } \left.\forall \mathrm{T} \in T_{\mathrm{h}}, \mu_{\mathrm{T}}=\underline{\mathrm{q}} \underline{\nu}_{-\mathrm{T}} \text { on } \partial \mathrm{T}\right\},
\end{gathered}
$$


where for this definition $q \cdot \nu_{T}$ denotes the normal trace on $\partial T$ of the restriction to $T$ of the vector function $q$. Thus $\underset{\sim}{q} \underline{\nu}_{T}$ is a priori an element of $H^{-\frac{1}{2}}(\partial \mathrm{T})$, cf. Remark 18.1 below.

Theorems 1.2 and 1.3 allow us to characterize $E\left(\partial T_{\mathrm{h}}\right)$ as being the subspace

$$
\begin{gathered}
\mathrm{E}\left(\partial T_{\mathrm{h}}\right)=\left\{\dot{\mu}=\left(\mu_{\mathrm{T}}\right) \mathrm{T} \in T_{\mathrm{h}} \in \mathrm{L}^{2}\left(\partial T_{\mathrm{h}}\right) ; \forall \mathrm{T}^{\prime}=\mathrm{T}_{1} \cap \mathrm{T}_{2}\right. \text { with } \\
\left.\mathrm{T}_{1}, \mathrm{~T}_{2} \in T_{\mathrm{h}^{\prime}}, \mu_{\mathrm{T}_{1}}+\mu_{\mathrm{T}_{2}}=0 \text { on } \mathrm{T}^{\prime}\right\}
\end{gathered}
$$

For the dual hybrid formulation the subspace of multipliers will be

$$
\begin{gathered}
\mathrm{L}\left(\partial T_{\mathrm{h}}\right)=\left\{\psi=\left(\psi_{\mathrm{T}}\right)_{\Gamma \in T_{\mathrm{h}}} \in \mathrm{L}^{2}\left(\partial T_{\mathrm{h}}\right) ; \exists \mathrm{v} \in \mathrm{H}^{1}(\Omega)\right. \\
\text { s.t. } \left.\forall T \in T_{h}, \quad \psi_{\mathrm{T}}=\mathrm{v} \text { on } \partial \mathrm{T}\right\},
\end{gathered}
$$

where in the expression $" \psi_{\Upsilon}=v$ on $\partial T^{*}, v$ denotes the trace on $\partial T$ of the restriction to $T$ of the function $v$. This trace is an element of $H^{\frac{1}{2}}(\partial T)$.

With Theorems 1.1 and 1.3 we may characterize $L\left(\partial T_{h}\right)$ as being the subspace

$$
\begin{aligned}
\mathrm{L}\left(\partial T_{\mathrm{h}}\right)= & \left\{\psi=\left(\psi_{\mathrm{T}}\right)_{\mathrm{T} \in T_{\mathrm{h}} \in \mathrm{T} \in T_{\mathrm{h}}} \mathrm{H}^{\frac{1}{2}}(\partial \mathrm{T}), \forall \mathrm{T}^{\prime}=\mathrm{T}_{1} \cap \mathrm{T}_{2}\right. \\
& \text { with } \left.\mathrm{T}_{1}, \mathrm{~T}_{2} \in T_{\mathrm{h}}, \quad \psi_{\mathrm{T}_{1}}=\psi_{\mathrm{T}_{2}} \text { on } \mathrm{T}^{\prime}\right\}
\end{aligned}
$$

Remark 18.1 . In fact the natural space of Lagrangian multipliers for the primal hybrid formulation is

$$
\begin{gathered}
E\left(\partial T_{\mathrm{h}}\right)=\left\{\mu=\left(\mu_{\mathrm{T}}\right) \mathrm{T} \in T_{\mathrm{h}} \in \underset{\mathrm{T} \in T_{\mathrm{h}}}{\mathrm{H}^{-\frac{1}{2}}(\partial \mathrm{T}) ; \exists \mathrm{q} \in \mathrm{H}(\operatorname{div} ; \Omega)}\right. \\
\text { s.t. } \left.\forall \mathrm{T} \in T_{\mathrm{h}}, \mu_{\mathrm{T}}=\underline{q} \cdot \underline{\nu}_{\mathrm{T}} \text { in } \mathrm{H}^{-\frac{1}{2}}(\partial \mathrm{T})\right\} .
\end{gathered}
$$

The choice (18.2) while more restrictive is less cumbersome and leads to no restriction for examples of finite element methods.

Remark 18.2. The first examples of hybrid formulations given in Section 3 were described as having as Lagrangian multipliers functions defined on all $\Omega$. Such a description is not in general well adapted for the numerical analysis of hybrid finite element methods. For hybrid formulations the Lagrangian 
multipliers are defined only on $\partial T_{h}$. It is sometimes possible to extend the multipliers to functions defined on all $\Omega$ while taking into account complementary information when necessary. Such a procedure is a post processing technique.

A primal hybrid formulation of problem (4.4) - (4.5) with the Robin's boundary condition (15.1) is given as follows : find a pair $(u, \lambda)$ satisfing

$$
\begin{cases}(u, \lambda) \in H^{1}\left(T_{h}\right) \times E\left(\partial T_{h}\right), & \text { for all } v \in H^{1}\left(T_{h}\right) \\ a(u, v)+b(v, \lambda)=\int_{\Omega} f v d x & \text { for all } \mu \in E\left(\partial T_{h}\right) \\ b(u, \mu)+d(\lambda, \mu)=-\sum_{T \in T_{h}} \int_{\partial T \cap} \frac{1}{t} g \mu_{T} d \sigma & \end{cases}
$$

where here

$$
\begin{aligned}
& a(u, v)=\sum_{T \in T_{h}} \sum_{i, j=1}^{n} \int_{T} a_{i j} \frac{\partial u}{\partial x_{j}} \frac{\partial v}{\partial x_{i}} d x, \\
& b(v, \mu)=-\sum_{T \in T_{h}} \int_{\partial T} v_{\mid T} \mu_{T} d \sigma,
\end{aligned}
$$

(in this expression, $v_{\mid T}$ denotes the trace on $\partial T$ of the restriction of $v$ to $T$ ) and

$$
\mathrm{d}(\lambda, \mu)=-\sum_{\mathrm{T} \in T_{\mathrm{h}}} \int_{\partial \mathrm{T} \cap \Gamma} \frac{1}{t} \lambda_{\mathrm{T}} \mu_{\mathrm{T}} \mathrm{d} \sigma .
$$

It is easy to show that problem (18.6) has at most one solution. If the solution ( $\mathrm{p}, \mathrm{u})$ of (15.2), which is also the solution of (15.8) and thus belongs to $H(\operatorname{div} ; \Omega) \times \mathrm{H}^{1}(\Omega)$, satisfies the regularity hypothesis that $\underset{\sim}{\mathrm{p}} \stackrel{\nu}{\nu}_{\mathrm{T}} \in \mathrm{L}^{2}(\partial \mathrm{T})$ for each $T \in T_{h}$, then the pair $\left(u,\left(\sim_{\sim} \cdot{ }_{T}\right) T \in T_{h}\right)$ is a solution of (18.6).

Thus we have

$$
\lambda_{T}=\stackrel{p}{p} \nu_{T} \quad \text { on } \partial T \quad \text { for each } T \in T_{h} .
$$

Remark 18.3. The spaces $H^{1}\left(T_{h}\right)$ and $L^{2}\left(\partial T_{h}\right)$ are supplied with Hilbert space structures in a natural way. With $W=\mathbf{H}^{1}\left(T_{h}\right)$ and with $M=E\left(\partial T_{h}\right)$ 
having the norm induced by that of $L^{2}\left(\partial T_{h}\right)$, we note that the bilinear form $b(\cdot, \cdot)$ does not satisfy the inf-sup condition on $W \times M$.

Toward the end of constructing an approximation $\left(u_{h}, \lambda_{h}\right)$ of the solution $(u, \lambda)$ of problem (18.6) we suppose that for each $T \in T_{h}$ we have two finite dimensional spaces $P_{T} \subset H^{1}(T)$ and $X_{T} \subset L^{2}(\partial T)$, and we put

$$
\mathrm{w}_{\mathrm{h}}=\left\{\mathrm{v} \in \mathrm{H}^{\mathrm{i}}\left(T_{\mathrm{h}}\right) ; \forall \mathrm{T} \in T_{\mathrm{h}}, \mathrm{v}_{\left.\mathrm{I}_{\mathrm{T}} \in \mathrm{P}_{\mathrm{T}}\right\}}\right\}
$$

and

$$
\mathbf{M}_{\mathrm{h}}=\left\{\mu=\left(\mu_{\mathrm{T}}\right)_{\mathrm{T} \in T_{\mathrm{h}}} \in \mathrm{E}\left(\partial T_{\mathrm{h}}\right) ; \forall \mathrm{T} \in T_{\mathrm{h}}, \mu_{\mathrm{T}} \in \mathrm{X}_{\mathrm{T}}\right\}
$$

We then seek $\left(u_{h}, \lambda_{h}\right)$ satisfying

$$
\begin{cases}\left(u_{h}, \lambda_{h}\right) \in W_{h} \times M_{h}, & \\ a\left(u_{h}, v_{h}\right)+b\left(v_{h}, \lambda_{h}\right)=\int_{\Omega} f v_{h} d x & \text { for all } v_{h} \in W_{h}, \\ b\left(u_{h}, \mu_{h}\right)+d\left(\lambda_{h}, \mu_{h}\right)=-\sum_{T \in T_{h}} \int_{\partial T \cap} \Gamma^{\frac{1}{t} g \mu_{h T} d \sigma} & \text { for all } \mu_{h} \in M_{h} .\end{cases}
$$

Remark 18.4. To treat the analogons problem with a Dirichlet boundary condition $u=\bar{u}$ on $\Gamma$, where $\bar{u}$ is a given function in $H^{\frac{1}{2}}(\Gamma)$ we would keep the same choice of subspaces $W_{h}$ and $M_{h}$ and look for $\left(u_{h}, \lambda_{h}\right)$ satisfying

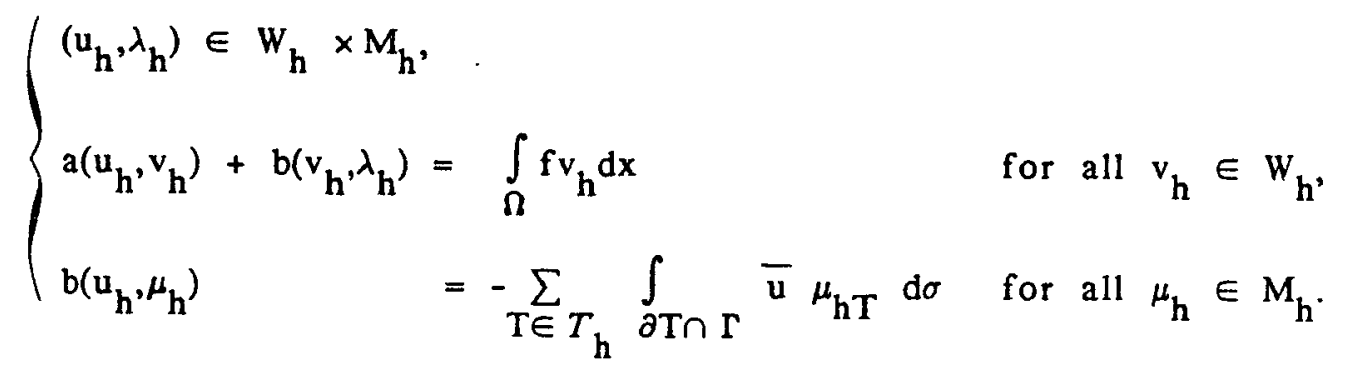

By contrast, to treat a homogeneous Neumann problem such as described in (14.1) or (14.6) we would introduce the subspace $M_{0 h}$ defined by

$$
{ }^{M_{0 h}}=\left\{\mu_{h} \in M_{h} ; V T \in T_{h}, \mu_{h T}=0 \text { ỗ̄ ôTก } \Gamma\right\}
$$

and look for $\left(u_{h}, \lambda_{h}\right)$ satisfying 


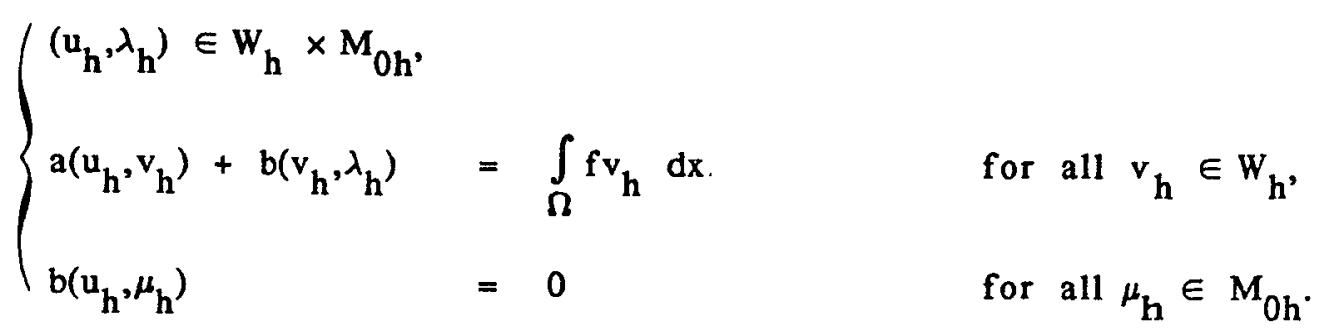

The bilinear form $a(\cdot, \bullet) \cdot$ is not $\mathbf{H}^{1}\left(T_{h}\right)$-elliptic and will not be $W_{h}$-elliptic. However, we shall introduce a hypothesis to make it $V_{h}$-elliptic, where

$$
v_{h}=\left\{v_{h} \in w_{h} ; \forall \mu_{h} \in M_{h}, b\left(v_{h}, \mu_{h}\right)=0\right\}
$$

THEOREM 18.1 . We suppose that the two subspaces $\mathrm{W}_{\mathrm{h}}$ and $\mathrm{M}_{\mathrm{h}}$ are compatible in the sense that the discrete inf-sup condition is satisfied:

$$
\left\{\mu_{h} \in M_{h} ; \forall v_{h} \in W_{h}, b\left(v_{h}, \mu_{h}\right)=0\right\}=\{0\}
$$

We suppose further that for each interface $\mathrm{T}^{\prime}=\mathrm{T}_{1} \cap \mathrm{T}_{2}$ with $\mathrm{T}_{1}, . \mathrm{T}_{2} \in T_{\mathrm{h}}$, there exists a function $\chi_{\mathrm{T}} \in \mathrm{L}^{2}\left(\mathrm{~T}^{\prime}\right)$ with $\int_{\mathrm{T}^{\prime}} \chi_{\mathrm{T}^{\prime}} \mathrm{d} \sigma \neq 0$ such that the function $\mathrm{\Lambda}=\left(\mathrm{\Lambda}_{\mathrm{T}}\right)_{\mathrm{T} \in T_{\mathrm{h}}}$ in E $\left(\partial T_{h}\right)$ defined by $\Lambda_{\mathrm{T}_{1}}=\chi_{\mathrm{T}^{\prime}}$ on $\mathrm{T}^{\prime}, \Lambda_{\mathrm{T}_{2}}=-\chi_{\mathrm{T}^{\prime}}$ on $\mathrm{T}^{\prime}$, and $\mathrm{\Lambda}_{\mathrm{T}}=0$ on all other faces for all $\mathrm{T} \in T_{\mathrm{h}^{\prime}}$, belongs to the subspace $\mathrm{M}_{\mathrm{h}}$. Similarily we suppose that for each boundary face $\mathrm{T} \subset \mathrm{C}$ of an element $\mathrm{T}_{\mathrm{o}} \in T_{\mathrm{h}}$, there exists a function $\chi_{\mathrm{T}^{\prime}} \in \mathrm{L}^{2}\left(\mathrm{~T}^{\prime}\right)$ with $\int_{\mathrm{T}^{\prime}} \chi_{\mathrm{T}^{\prime}} \mathrm{d} \sigma \neq 0$ such that the function $\mathrm{\Lambda}=\left(\Lambda_{\mathrm{T}}\right) \mathrm{T} \in T_{\mathrm{h}}$ in $\mathrm{E}\left(\partial \mathrm{T}_{\mathrm{h}}\right)$ defined by $\Lambda_{\mathrm{T}_{\mathrm{o}}}=\chi_{\mathrm{T}}$, on $\mathrm{T}^{\prime}$ and $\Lambda_{\mathrm{T}}=0$ on all other faces for all $\mathrm{T} \in T_{\mathrm{h}}$, belongs to the subspace $\mathrm{M}_{\mathrm{h}}$. Then problem (18.13) has a unique solution.

Proof. Each function $v_{h} \in v_{h}$ satisfying $a\left(v_{h}, v_{h}\right)=0$ is a function whose restriction to $T$, for each $T \in T_{h}$, is constant. The first part of the additional hypothesis guarantees that such a function may be identified with a function constant on each component of $\Omega$. The second part forces such a function to vanish on $\Gamma=\partial \Omega$. We conclude that the form $a(\cdot, \cdot)$ is $V_{h}$-elliptic and, in light of Remark 10.1, that the theorem follows.

It is important to note that the compatibility condition (18.15) that should be satisfied by the subspaces $W_{h}$ and $M_{h}$ given in the forms (18.11) and (18.12) is satisfied as soon as, for each $T \in T_{h}$, the spaces $P_{T}$ and $X_{T}$ satisfy the local compatibility condition 
(18.16)

$$
\left\{\mu \in \mathrm{X}_{\mathrm{T}} ; \forall \mathrm{v} \in \mathrm{P}_{\mathrm{T}^{\prime}} \int_{\partial \mathrm{T}} \mu \mathrm{v} \mathrm{d} \sigma=0\right\}=\{0\}
$$

By contrast, the additional hypotheses of Theorem 18.1 demand that the functions belonging to $V_{h}$ have a minimum amount of continuity at the interfaces of the triangulation. In the example that follows this continuity hypothesis will be trivially satisfied. (We will in fact choose $\chi_{T}$, to be a constant function.)

THEOREM 18.2. Let $\Omega$ be an open polygonal domain in $\mathrm{R}^{2}$ triangulated by triangles $\mathrm{T}$. Let $\mathrm{k}$ be a positive integer, and put

$$
\mathrm{k}^{*}= \begin{cases}\mathrm{k} & \text { if } \mathrm{k} \text { is odd } \\ \mathrm{k}+1 & \text { if } \mathrm{k} \text { is even. }\end{cases}
$$

For each triangle $\mathrm{T} \in T_{\mathrm{h}}$, define the subspace $\mathrm{X}_{\mathrm{T}} \subset \mathrm{L}^{2}(\partial \mathrm{T})$ by

$$
X_{T}=D_{k}(\partial T)
$$

(With the definition of the space $\mathrm{E}_{\partial T_{\mathrm{h}}}^{(\mathrm{k})}$ given by (6.30), we have $\mathrm{M}_{\mathrm{h}}=\mathrm{E}_{\partial T_{\mathrm{h}}}^{(\mathrm{k})}$ ). Suppose that the subspace $\mathrm{P}_{\mathrm{T}} \subset \mathrm{H}^{1}(\mathrm{~T})$ is chosen such that the following property is satisfied:

$$
\forall \mathbf{w} \in \mathrm{P}_{\mathbf{k}^{*}} *(\partial \mathrm{T}), \exists v \in \mathrm{P}_{\mathrm{T}} \text { s.t. } \mathbf{v}=\mathbf{w} \text { on } \partial \mathrm{T} \text {. }
$$

Then problem (18.13) has a unique solution.

Proof. The elements of the space $\mathrm{D}_{k}(\partial \mathrm{T})$ defined in (6.32) are polynomials of degree $\leq k-1$ on each edge. It is clear that condition (18.16) is satisfied if each element of $P_{k+1}(\partial T)$ vanishing at the vertices of $T$ is the trace of a function $v \in P_{T}$. Even though we have $\operatorname{dim} P_{k}(\partial T)=\operatorname{dim} D_{k}(\partial T)$, we know (cf. RAVIART \& THOMAS $\left(1977 \mathrm{~b}\right.$, lemma 4)) that the subspace of $D_{k}(\partial T)$ consisting of all those functions $\mu \in D_{k}(\partial T)$ satisfying $\int_{\partial T} \mu w d \sigma=0$ for each $w \in P_{k}(\partial T)$ is trivial only if $k$ is odd. It is of dimension one when $k$ is even.

Before giving an error estimate we need to make precise our choice of norms. For the sake of simplicity we suppose here that the triangulation is uniformly regular. To each function $\mathrm{v} \in \mathrm{H}^{1}\left(T_{\mathrm{h}}\right)$ we associate the function $\delta \mathrm{v} \in$ $L^{2}\left(\partial T_{h}\right)$, constant on each edge $T^{\prime} \in \partial T_{h}$, defined by 


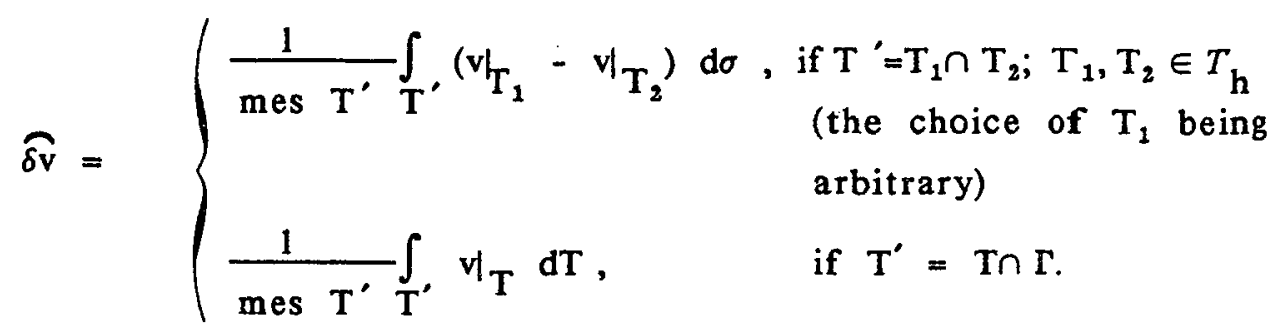

Once the hypotheses of Theorem 18.1 are satisfied, we clearly have $\delta v=0$ if $v \in H_{0}^{1}(\Omega) \oplus V_{h}$. The space $L^{2}\left(\partial T_{h}\right)$ is given the norm

$$
\|\mu\|_{0, \partial T_{h}}=\left\{\sum_{\mathrm{T} \in T_{\mathrm{h}}}\left\|\mu_{\mathrm{T}}\right\|_{0, \partial \mathrm{T}}^{2}\right\}^{\frac{1}{2}},
$$

the space $\mathrm{H}^{1}\left(T_{h}\right)$ the norm

$$
[\mathrm{v}]_{1, T_{\mathrm{h}}}=\left\{\sum_{\mathrm{T} \in T_{\mathrm{h}}}|\mathrm{v}|_{1, \mathrm{~T}^{+}}^{2} \mathrm{~h}^{-1}\|\hat{\delta \mathrm{v}}\|_{0, \partial T_{\mathrm{h}}^{2}}^{2}\right\}^{\frac{1}{2}},
$$

and the space $E\left(\partial T_{h}\right)$ the norm $\|1 \cdot\| \|_{-\frac{1}{2}, \partial T_{h}}$ defined in (6.36). One can show (by adapting, for example, the demonstration of Theorem V.4.3 in THOMAS (1977)) that the Poincaré-Friedrichs inequality,

$$
\|\mathrm{v}\|_{0, \Omega} \leq \mathrm{C} \quad[\mathrm{v}]_{1, T_{\mathrm{h}}} \quad \text { for all } \mathrm{v} \in \mathrm{H}^{1}\left(T_{\mathrm{h}}\right)
$$

is satisfied with a constant $C$ independent of $h$. (Note that the two norms on $\mathrm{H}^{1}\left(T_{\mathrm{h}}\right), \mathrm{v} \rightarrow[\mathrm{v}]_{1, T_{\mathrm{h}}}$ and $\mathrm{v} \longrightarrow\|\mathrm{v}\| \|_{1, T_{\mathrm{h}}}=\left(\sum_{\mathrm{T} \in T_{\mathrm{h}}}\left(\|\mathrm{v}\|_{1, \mathrm{~T}}^{2}+\mathrm{h}^{-2}\|\mathrm{v}\|_{0, \mathrm{~T}}^{2}\right)^{\frac{1}{2}}\right.$, are equivalent uniformly in $h$.) One may also verify that there exist constants $c$ and $C_{k}$ independent of $h$ such that

$$
\|I \mu\|\left\|_{-\frac{1}{2}}, \partial T_{h} \quad \leq c h^{\frac{1}{2}}\right\| \mu \|_{0, \partial T_{h}} \quad \text { for all } \mu \in L^{2}\left(\partial T_{h}\right) \text {, }
$$

and

$$
\left\|\mu_{\mathrm{h}}\right\|_{0, \partial T_{\mathrm{h}}} \quad \leq \mathrm{C}_{\mathrm{k}} \mathrm{h}^{-\frac{1}{2}}\|\mu\| \|_{-\frac{1}{2}, \partial T_{\mathrm{h}}} \text { for all } \mu_{\mathrm{h}} \in \mathrm{E}_{\partial T_{\mathrm{h}}}^{(\mathrm{k})} \text {. }
$$

THEOREM 18.3. We suppose the hypotheses of Theorem 18.2 except here we fix for each $\mathrm{T} \in T_{\mathrm{h}}$

$$
\mathrm{X}_{\mathrm{T}}=\mathrm{D}_{\mathrm{k}}(\partial \mathrm{T}), \quad \mathrm{P}_{\mathrm{T}}=\mathrm{P}_{\mathbf{k}^{*}}(\mathrm{~T})
$$

Then there exists a constant $\mathrm{C}$ independant of $\mathrm{h}$ such that we have

$$
\left[u-u_{h}\right]_{1, T_{h}}+\|\left. I\left|\lambda-\lambda_{h}\right| I\right|_{-\frac{1}{2}, \partial T_{h}} \leq C^{k}\left\{|u|_{k+1, \Omega}+|\underset{g}{k}|_{k, \Omega}\right\}
$$


once the solution $(\mathrm{p}, \mathrm{u})$ of (15.2) and of (15.8) belongs to $\left(\mathrm{H}^{\mathrm{k}}(\Omega)\right)^{2} \times \mathrm{H}^{\mathrm{k}+1}(\Omega) ;(\lambda$ is expressed as a function of $\mathrm{p}$ in (18.10).)

Proof. It is clear that the bilinear form $a(\cdot, \cdot)$ satisfies

$$
\mathrm{a}(\mathrm{u}, \mathrm{v}) \leq \mathrm{A} \quad[\mathrm{u}]_{1, T_{\mathrm{h}}}[\mathrm{v}]_{1, T_{\mathrm{h}}} \quad \text { for all } \mathrm{u}, \mathrm{v} \in \mathrm{H}^{1}\left(T_{\mathrm{h}}\right)
$$

and

$$
a\left(v_{h}, v_{h}\right) \geq \alpha_{p}\left[v_{h}\right]_{1, T_{h}}^{2} \quad \text { for all } v_{h} \in v_{h} \text {, }
$$

as $v_{h} \in V_{h}$ implies $\hat{\delta v}_{h}=0$. We can also establish the existence of a constant $\beta, \beta>0$, such that

$$
\sup _{v_{h} \in W_{h}} \frac{b\left(v_{h}, \mu\right)}{\left[v_{h}\right]_{1, \Omega}} \geq \beta\|\| \mu_{h} \| I_{-\frac{1}{2}, \partial T_{h}} \quad \text { for all } \mu_{h} \in M_{h}
$$

Finally we show the existence of a constant $B_{0}$ such that

$$
\begin{aligned}
& \mathrm{b}(\mathrm{v}, \mu) \leq \mathrm{B}_{0}[\mathrm{v}]_{1, T_{\mathrm{h}}} \quad\|\| \mu\|\|_{-\frac{1}{2}, \partial T_{\mathrm{h}}} \quad \text { for all } \mathrm{v} \in \mathrm{H}^{1}\left(T_{\mathrm{h}}\right) \\
& \text { and all } \mu \in \mathrm{E}_{0}\left(\partial T_{\mathrm{h}}\right) \text {, }
\end{aligned}
$$

where $\mathrm{E}_{0}\left(\partial T_{\mathrm{h}}\right)$ is the subspace of functions $\mu \in \mathrm{E}\left(\partial T_{\mathrm{h}}\right)$ such that $\int_{\mathrm{T}} \mu \mathrm{d} \sigma=0$ on each edge $T{ }^{\prime} \subset r$. Now, for a Dirichlet boundary condition or for a Neumann boundary condition, using a variant of Theorem 10.4, we can establish the existence of a constant $C$, independent of $h$, such that

$$
\begin{aligned}
& {\left[u-u_{h}\right]_{1, T_{h}}+\|\| \lambda-\lambda_{h}\|\|_{-\frac{1}{2}, \partial T_{h}} \leq C\left\{\inf _{v_{h} \in W_{h}}\left[u-v_{h}\right]_{1, T_{h}}\right.}
\end{aligned}
$$

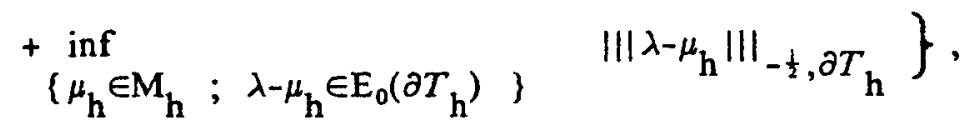

and then using (6.37) deduce the inequality (18.26).

To handle the case of Robin's boundary condition we introduce the direct sum decomposition of $M=E\left(\partial T_{h}\right)$ in the form $M=M_{0} \oplus \bar{M}$, where $M_{0}$ is the subspace of all $i \in M$ such that for each $T \in T_{h}$ with $T \cap \Gamma \neq \phi, \mu_{T}=0$ on each edge $T^{\prime}$ contained in the boundary $\Gamma$. Problem (18.6), respectively (18.13), may be put in the abstract form (10.1), respectively $(10.6)$, posed in $\left(\mathrm{H}^{1}\left(T_{\mathrm{h}}\right) \times\right.$ $\bar{M}) \times M_{0}$, with for bilinear forms 


$$
(u, \bar{\lambda}),(v, \bar{\mu}) \longrightarrow a(u, v)+b(v, \bar{\lambda})-b(u, \bar{\mu})-d(\bar{\lambda}, \bar{\mu})
$$

and

$$
(\mathrm{v}, \bar{\mu}), \mu_{0} \longrightarrow \mathrm{b}\left(\mathrm{v}, \mu_{0}\right)
$$

We then deduce as above

$$
\begin{gathered}
{\left[u-u_{h}\right]_{1, T_{h}}+\left\|\bar{\lambda}-\bar{\lambda}_{h}\right\|_{0, \partial T}+\mid\left\|\lambda_{0}-\lambda_{o h} \cdot\right\|_{-\frac{1}{2}, \partial T_{h}}} \\
\leq C h^{k}\left\{|u|_{k+1, \Omega}+|\underline{p}|_{k, \Omega}\right\} .
\end{gathered}
$$

Remark 18.5 - If the integer $\mathrm{k}$ is even, then $\mathrm{k}^{*}=\mathrm{k}+1$, and the choice $\mathrm{P}_{\mathrm{T}}=$ $P_{k+1}(T)$ is not optimal. We can obtain the same results by taking $P_{T}$ to be the space generated by $P_{k}(T)$ together with an appropriately choosen element of $\mathrm{P}_{\mathrm{k}+1}(\mathrm{~T})$. For more details see RAVIART \& THOMAS (1977b).

The primal hybrid finite element method corresponding to the subspaces $M_{h}$ and $W_{h}$ associated with the spaces $X_{T}$ and $P_{T}$ of (18.25) by (18.11) and (18.12) is convergent for each positive integer $k$, in the following sense : we can show, in the absence of any hypothesis concerning the regularity of the solution $u$, that

$$
\lim _{h \rightarrow 0}\left\|u-u_{h}\right\|_{0, \Omega}=0
$$

Approximation methods based on a principle analogous to that which was the basis for the development of primal hybrid finite element methods have been studied by BABUSKA (1973), BRAMBLE (1981), FALK (1976), and PITKÄRANTA (1979), (1980a), (1981). For a problem with a nonhomogeneous Dirichlet boundary condition $u=\tilde{u}$ on $\Gamma$, we dualize the boundary condition using a multiplier $\lambda \in \mathrm{H}^{-\frac{1}{2}}(\Gamma)$. The discrete inf-sup condition is established by means of compactness arguments; thus the results, notably existence and uniqueness, are assured only for "h sufficiently small". 


\section{PRIMAL HYBRID METHODS AND NONCONFORMING METHODS}

We consider the model Dirichtlet problem

$$
\left\{\begin{aligned}
-\Delta u & =\mathbf{f} & & \text { in } \Omega, \\
\mathbf{u} & =\bar{u} & & \text { on } \Gamma,
\end{aligned}\right.
$$

where $f$ is a given function in $L^{2}(\Omega), \bar{u}$ a given function in $H^{\frac{1}{2}}(\Gamma)$, and $\Omega$ a bounded, open domain in $\mathbb{R}^{2}$ with polygonal boundary $\Gamma$. For a triangulation $T_{\mathrm{h}}$ of $\Omega$ by triangles, the simplest primal hybrid finite element method for solving this problem is given as follows : find $\left(u_{h}, \lambda_{h}\right)$ satisfying

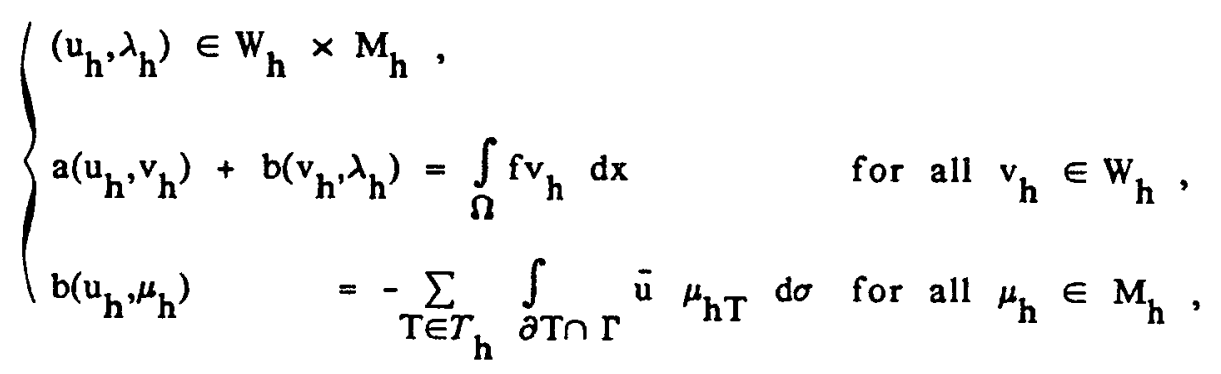

with

and

$$
\begin{aligned}
& \mathrm{W}_{\mathrm{h}}=\left\{\mathrm{v} \in \mathrm{H}^{1}\left(T_{\mathrm{h}}\right) ; \forall \mathrm{T} \in T_{\mathrm{h}}, \mathrm{v}_{\mathrm{I}_{\mathrm{T}}} \in \mathrm{P}_{1}(\mathrm{~T})\right\},
\end{aligned}
$$

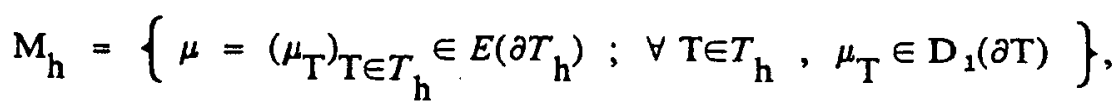

$$
\begin{aligned}
& \mathrm{a}(\mathrm{u}, \mathrm{v})=\sum_{\mathrm{T} \in T_{\mathrm{h}}} \int_{\mathrm{T}} \underset{\sim}{\operatorname{grad}} \mathrm{u} \cdot \underset{\sim}{\operatorname{grad}} \mathrm{vdx}, \\
& \mathrm{b}(\mathrm{v}, \mu)=-\sum_{\mathrm{T} \in T_{\mathbf{h}}} \int_{\partial \mathrm{T} \cap \Gamma}{ }^{\mu} \mathrm{T} \mathrm{v}_{\mathrm{TT}} \mathrm{d} \sigma .
\end{aligned}
$$

Eliminating the Lagrangian multiplier, we obtain a characterization of $u_{h}$ as the solution of the variational problem

$$
\left\{\begin{array}{l}
u_{h} \in v_{h}^{\bar{u}}, \\
a\left(u_{h}, v_{h}\right)=\int_{\Omega} f_{v_{h}} d x
\end{array}, \quad \text { for all } v_{h} \in v_{h},\right.
$$


with

$$
v_{h}^{\bar{u}}=\left\{v_{h} \in w_{h} ; \forall \mu_{h} \in M_{h} \quad b\left(v_{h}, \mu_{h}\right)=-\sum_{T \in T_{h}} \int_{\partial T \cap \Gamma} \bar{u} \mu_{h T} d \sigma\right\}
$$

and

$$
v_{h}=\left\{v_{h} \in w_{h} ; \forall \mu \in M_{h}, b\left(v_{h}, \mu_{h}\right)=0\right\}
$$

It is a simple matter to check that $v_{h}^{\bar{u}}$ is the set of functions in $W_{h}$ that are affine on each triangle $T \in T_{h}$, are continuous at the midpoint of each edge $T^{\prime}$ common to two triangles $T_{1}$ and $T_{2}$ of $T_{h}$ and have for value at the midpoint of each boundary edge $T^{\prime} \subset \Gamma$ the average value of $\bar{u}$ on $T$. The set of midpoints of the three sides of a triangle is $P_{1}$-unisolvent. Thus a function $v_{h}$ $\in V_{h}$ is uniquely determined by its values at the midpoints of the edges common to two triangles of $T_{h}$. It is not difficult to give a basis of $V_{h}$ associated with this choice of degrees of freedom for a function in $V_{h}$. Thus, problem (19.2) can be solved directly. Such a procedure is said to be a nonconforming finite element method since $V_{h}$ is not a subspace of $V=H_{0}^{1}(\Omega)$, the space of test functions for the primal variational formulation.

If the solution $u$ belongs to $\mathrm{H}^{2}(\Omega)$ we can obtain, using the results of the previous section, the error bound

$$
\left\{\sum_{T \in T_{h}}\left|u-u_{h}\right|_{1, T}^{2}\right\}^{\frac{1}{2}}+\left\|u-u_{h}\right\|_{0, T} \leq C h|u|_{2, \Omega},
$$

an estimate that is classically obtained in a direct manner, cf. STRANG (1972) or STRANG \& FIX (1973, Chapter 4.2).

After having calculated the solution of (19.2), to obtain the solution of (19.1) we have only to determine $\lambda_{h} \in M_{h}$ satisfying

$$
b\left(v_{h}, \lambda_{h}\right)=\int_{\Omega} f v_{h} d x-a\left(u_{h}, v_{h}\right) \quad \text { for each } v_{h} \in w_{h},
$$

i.e. the functions $\lambda_{h T}$, constant on each edge of $T$, are determined by

$$
\int_{\partial T} v \lambda_{h T} d \sigma=\int_{T} f v d x-\int_{T} \underset{\sim}{\operatorname{grad}} u_{h} \cdot \operatorname{grad} v d x \text { for each } v \in P_{1}(T) .
$$

Thus once the nonconforming approximation $u_{h}$ of $u$ has been calculated, a postprocessing procedure furnishes directly an approximation of the flux of grad $u$ across each edge $T^{\prime}$. If $T^{\prime}$ is the edge common to $T_{1}$ and $T_{2} \in T_{h}$, then the values $\lambda_{h T_{1}}$ and $\lambda_{h T_{2}}$ obtained from (19.4) satisfy 


$$
\lambda_{h T_{1}}+\lambda_{h T_{2}}=0, \quad \text { on } T^{\circ}=T_{1} \cap T_{2}
$$

The flux across $T^{\prime}$ from $T_{1}$ into $T_{2}$ is minus that from $T_{2}$ into $T_{1}$.

More elaborate examples of nonconforming finite elements derived from primal hybrid finite element methods for second order elliptic problems on an open set $\Omega$ in $\mathbb{R}^{2}$ may be found in RAVIART \& THOMAS (1977b). For those examples the continuity of the functions of $V_{h}$ is imposed at the Gauss points of the edges $T^{\prime}$. The example treated above, where the functions $v_{h}$ are locally affine, is readily generalized to the 3-dimensional case, where $\Omega$ is an open set of $\mathbb{R}^{3}$. However the situation quickly becomes more difficult for higher orders of approximation. An example with $k=2$ is given in FORTIN (1985).

In fact nonconforming finite element methods were first developed for elliptic problems more complicated than the model problem above, e.g. :

1) a fourth order elliptic problem where the space $V$ is a subspace of $\left(\mathrm{H}^{2}(\Omega)\right)^{2}$ and is thus made up of functions of class $C^{1}$ on $\bar{\Omega}$. Examples and analyses of such methods may be found in CIARLET (1974), (1978, pp. 362-380), CIAVALDINI \& NEDELEC (1974), and ZHONG-CI SHI (1984a).

2) Stokes problem formulated as a variational problem on a subspace of the space $V$ of divergence free functions in $\left(H^{1}(\Omega)\right)^{n}$. Analyses of such methods are given in CROUZEIX \& RAVIART (1973), FORTIN (1981), HECHT (1981) and TEMAM (1977, pp. 172-181).

3) a problem of linear elasticity formulated as a variational problem on a subspace of $\mathrm{V}=\left(\mathrm{H}^{1}(\Omega)\right)^{\mathrm{n}}$. The motivation in this case is to obtain models less rigid than those provided by conforming methods, of. in particular PIAN (1971) (1972), FRAEIJS DE VEUBEKE (1974b). The most popular noncorforming finite element for this type of problem is Wilson's brick (WILSON et al. (1973)). An analysis of the method may be found in LESAINT (1976), LESAINT \& ZLAMAL (1980), ZHONH-CI SHI (1984b) or as a particular case of primal hybrid methods in THOMAS $(1977, \mathrm{Ch}$. VI). Other interpretations using hybrid methods have been proposed in IRONS (1972), PIAN \& TONG (1986).

We conciude this section by pointing out that the probiem of finding necessary and sufficient conditions for convergence of a nonconforming finite element method has been the subject of many discussions between theoreticians and engineers, $c f$. in particular the response of IRONS \& LOIKKANEN (1983) to STUMMEL (1979), (1980a), and (1980b). When a nonconforming method can be 
interpreted as a primal hybrid method, possibly with reduced numerical integration, the study of its convergence is based in particular on the verification of the discrete inf-sup condition.

\section{EXAMPLES OF DUAL HYBRID FINITE ELEMENT METHODS}

Let $T_{h}$ be a triangulation of the domain $\bar{\Omega}$. Associated to this triangulation are the spaces $H\left(\operatorname{div} ; T_{h}\right)$ defined by $(6.16)$ and $\mathrm{L}\left(\partial T_{h}\right)$ introduced in (18.4) and (18.5). Given a function $f \in L^{2}(\Omega)$ denote by $Q^{f}\left(T_{h}\right)$ the affine manifold defined by

$$
\mathrm{Q}^{\mathrm{f}}\left(T_{\mathrm{h}}\right)=\left\{\underline{\mathrm{q}} \in H\left(\operatorname{div} ; T_{\mathrm{h}}\right) ; \forall \mathrm{T} \in T_{\mathrm{h}}, \operatorname{div}\left({\underline{q_{1}}}_{\mathrm{T}}\right)+\mathrm{f}_{\left.\right|_{\mathrm{T}}}=0\right\}
$$

or more precisely defined as the space of vector functions $q \in H\left(\right.$ div; $\left.T_{h}\right)$ which satisfy $\int_{\Omega} \underset{q}{q} \underset{\sim}{\operatorname{grad}} v \mathrm{dx}=\int_{\Omega} \mathrm{fvdx}$, for each function $v \in \mathrm{H}^{1}(\Omega)$ whose trace on $\partial T_{h}$ is trivial. In particular, $\mathrm{Q}^{0}\left(T_{\mathrm{h}}\right)$ is the vector space of functions $q \in$ $H\left(\operatorname{div} ; T_{\mathrm{h}}\right)$ for which ${ }_{-}^{q_{\mathrm{T}}}$ is divergence free for each $\mathrm{T} \in T_{\mathrm{h}}$.

A dual mixed hybrid formulation of our model problem (4.4), (4.5) with Robin's boundary condition (15.1) is given as follows : find a pair (p, $\phi)$ satisfying

$$
\begin{cases}(\underline{p}, \phi) \in Q^{f}\left(T_{h}\right) \times L\left(\partial T_{h}\right), & \\ a(\underline{p}, \underline{q})+b(\underline{q}, \phi)=0 & \text { for all } \underline{q} \in Q^{0}\left(T_{h}\right) \\ b(\underline{p}, \psi)+d(\phi, \psi)=-\sum_{T \in T_{h}} \int_{\partial T \cap \Gamma} g \psi_{T} d \sigma & \text { for all } \psi \in L\left(\partial T_{h}\right)\end{cases}
$$

with here

$$
\begin{aligned}
& a(\underset{\sim}{p}, \underset{\sim}{q})=\sum_{i, j=1}^{n} \int_{T} A_{i j} p_{j} q_{i} d x, \\
& b(\underline{q}, \psi)=-\sum_{T \in T_{h}} \int_{\partial T} \psi_{T} \stackrel{q}{q} \cdot \underline{v}_{T} d \sigma,
\end{aligned}
$$

(in this last expression, $q \cdot \nu_{\sim} T$ denotes the normal trace on $\partial \mathrm{T}$ of the restriction of $q$ to $T$ ), and

$$
\mathrm{d}(\phi, \psi)=-\sum_{\mathrm{T} \in T_{\mathrm{h}}} \int_{\partial \mathrm{T} \cap \Gamma} \mathrm{t} \phi_{\mathrm{T}} \psi_{\mathrm{T}} \mathrm{d} \sigma .
$$


After noting that, for each $T \in T_{h}$, the normal trace on the boundary of $\mathrm{T}$ is a surjective mapping of $\{\underline{\mathrm{q}} \in H(\mathrm{div} ; \mathrm{T}) ; \operatorname{div} \underline{q}=0\}$ onto $\left\{\mu \in \mathrm{L}^{2}(\partial \mathrm{T}) ; \int_{\partial \mathrm{T}} \mu \mathrm{d} \sigma=0\right\}$, it is easy to verify that problem $(20.2)$ has at most one solution. One shows that if the solution (p,u) of (15.2) and of (15.8) belongs to $\left(H(\operatorname{div} ; \Omega) \cap H\left(\operatorname{div} ; T_{h}\right)\right) \times H^{1}(\Omega)$, (this is the same regularity hypothesis as that required for the existence of a solution to the primal hybrid problem (18.6)), then the pair ( $\left.\underset{p}{ },\left(u_{\partial T}\right)_{T \in T_{h}}\right)$ is a solution of (20.2). One thus has

$$
\phi_{T}=u \quad \text { on } \partial T \quad \text { for all } T \in T_{h}
$$

Remark 20.1. If we equip the spaces $H\left(\operatorname{div} ; T_{h}\right)$ and $\mathrm{L}\left(\partial T_{\mathrm{h}}\right)$ with Hilbert space structures, then $b(\cdot, \cdot)$ considered as a bilinear form on $W \times M$, with $W=Q^{0}\left(T_{h}\right)$ a subspace of $H\left(\operatorname{div} ; T_{h}\right)$ and $\mathrm{M}=\mathrm{L}\left(\partial T_{\mathrm{h}}\right)$, does not satisfy the inf-sup condition. (This situation is analagous to the case of the primal hybrid formulation, cf. Remark 18.3.)

To construct an approximation of the solution $(p, \phi)$ of problem (20.2), we take for each $\mathrm{T} \in T_{\mathrm{h}}$ two finite dimensional subspaces, $\mathrm{Q}_{\mathrm{T}} \subset H(\operatorname{div} ; \mathrm{T})$ and $\mathrm{Y}_{\mathrm{T}}$ $\subset \mathrm{H}^{\frac{1}{2}}(\partial T)$. To a given function $f \in L^{2}(\Omega)$, we associate the function $f_{h} \in L^{2}(\Omega)$ whose restriction to $T$, for each $T \in T_{h}$, is the orthogonal projection in $L^{2}(T)$ of $\mathrm{f}_{\mathrm{T}_{\mathrm{T}}}$ into the finite dimensional space $\left\{\operatorname{div} \mathrm{q} ; \mathrm{q} \in \mathrm{Q}_{\mathrm{T}}\right\}$. We then put

$$
\begin{aligned}
& \mathrm{w}_{\mathrm{h}}^{\mathrm{f}}=\left\{\underline{\mathrm{q}} \in H\left(\operatorname{div} ; T_{\mathrm{h}}\right) ; \forall \mathrm{T} \in T_{\mathrm{h}}, \underline{\mathrm{q}}_{-1} \in \mathrm{Q}_{\mathrm{T}}\right\} \cap \mathrm{Q}^{\mathrm{f}_{\mathrm{h}}}\left(T_{\mathrm{h}}\right), \\
& \mathrm{W}_{\mathrm{h}}=\mathrm{W}_{\mathrm{h}}^{0}=\left\{\mathrm{q} \in H\left(\operatorname{div} ; T_{\mathrm{h}}\right) ; \forall \mathrm{T} \in T_{\mathrm{h}}, \stackrel{\mathrm{q}}{-} / \mathrm{T}_{\mathrm{T}} \in \mathrm{Q}_{\mathrm{T}}\right\} \cap \mathrm{Q}^{\circ}\left(T_{\mathrm{h}}\right),
\end{aligned}
$$

and

$$
\mathrm{M}_{\mathrm{h}}=\left\{\psi=\left(\psi_{\mathrm{T}}\right)_{\mathrm{T} \in T_{\mathrm{h}}} \in \mathrm{L}\left(\partial T_{\mathrm{h}}\right) ; \forall \mathrm{T} \in T_{\mathrm{h}}, \psi_{\mathrm{T}} \in \mathrm{Y}_{\mathrm{T}}\right\}
$$

We seek a pair $\left(p_{h}, \phi_{h}\right)$ satisf ying

$$
\begin{cases}\left(\underline{p}_{h}, \phi_{h}\right) \in w_{h}^{f} \times M_{h}, & \\ a\left(\underline{p}_{h}, q_{h}\right)+b\left(\underline{q}_{h}, \phi_{h}\right)=0 & \text { for all } \underline{q}_{h} \in W_{h}, \\ b\left(\underline{p}_{h}, \psi_{h}\right)+d\left(\phi_{h}, \psi_{h}\right)=-\sum_{T \in T_{h}} \int_{\partial T \cap \Gamma} g \psi_{h T} d \sigma & \text { for all } \psi_{h} \in M_{h} .\end{cases}
$$


Remark 20.2 . The analagous problem with a Neumann boundary condition p. $\nu=g$, where $g$ is a given function in $L^{2}(\Gamma)$ satisfying $\int_{\Omega} f d x+\int_{\Gamma} g d \sigma=0$, is formulated as follows : find a pair $\left(\mathfrak{\sim}_{h}, \phi_{h}\right)$ satisfying

$$
\begin{cases}\left(\stackrel{p}{h}_{h}, \phi_{h}\right) \in W_{h}^{f} \times M_{h}, & \\ a\left(\underline{p}_{h}, \underline{q}_{h}\right)+b\left(\underline{q}_{h}, \phi_{h}\right)=0 & \text { for all } \underline{q}_{h} \in W_{h}, \\ b\left(\underline{p}_{h}, \psi_{h}\right)=-\sum_{T \in T_{h}} \int_{\partial T \cap \Gamma} g \psi_{h T} d \sigma & \text { for all } \psi_{h} \in M_{h},\end{cases}
$$

where $w_{h}^{f}, w_{h}$, and $M_{h}$ are as defined in (20.7), (20.8), and (20.9).

To treat the homogeneous Dirichlet problem we seek a solution $\left(\mathrm{\sim}_{h}, \phi_{h}\right)$ of the following problem :

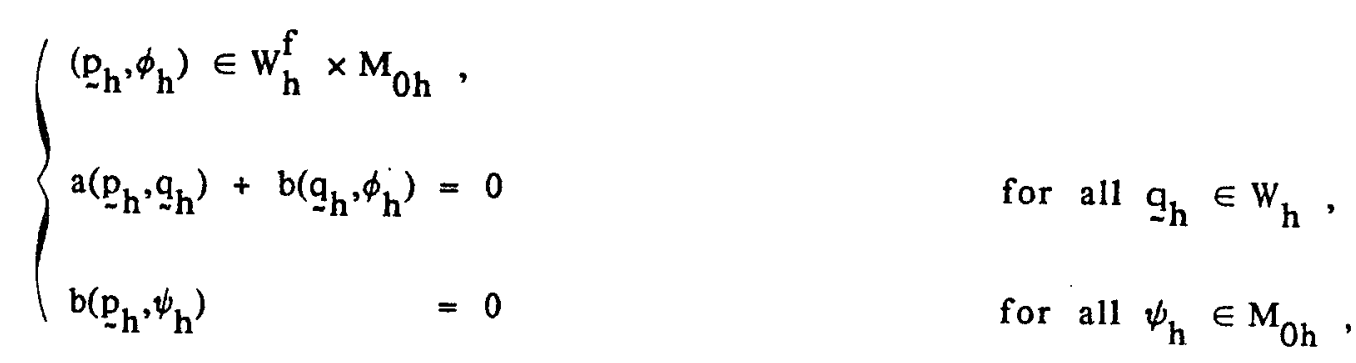

where $M_{0 h}$ is the subspace ot $M_{h}$ defined by

$$
\mathrm{M}_{0 \mathrm{~h}}=\left\{\psi_{\mathrm{h}} \in \mathrm{M}_{\mathrm{h}} ; \forall \mathrm{T} \in T_{\mathrm{h}}, \psi_{\mathrm{hT}}=0 \text { on } \partial \mathrm{T} \cap \Gamma\right\}
$$

By construction of the function $f_{h}$, the affine manifold $w_{h}^{f}$ is not empty. Applying Theorem 10.2 with Remark 10.7 we obtain with no difficulty the following theorem :

THEOREM 20.1. Problem (20.10) has a unique solution if and only if the subspaces $\mathrm{W}_{\mathrm{h}}$ and $\mathrm{M}_{\mathrm{h}}$ are compatible in the sense that the discrete inf-sup condition is satisfied :

$$
\left\{\psi_{h} \in M_{0 h} ; \forall \underline{q}_{h} \in W_{h}, b\left(\underline{q}_{h}, \psi_{h}\right)=0\right\}=\{0\}
$$

where $\mathrm{M}_{0 \mathrm{~h}}$ is the subspace of $\mathrm{M}_{\mathrm{h}}$ defined in (20.11). 
The global condition (20.12) is satisfied once, for each $T \in T_{h}$, the choice of spaces $Q_{T}$ and $Y_{T}$ satisfy the local compatibility condition

$$
\left\{\psi \in \mathrm{Y}_{\mathbf{T}} ; \forall \underline{q} \in \mathrm{Q}_{\mathbf{T}} \text { with } \operatorname{div} \underline{\mathrm{q}}=0, \int_{\partial \mathrm{T}} \psi \underline{q} \cdot \nu_{\mathrm{T}} \mathrm{d} \sigma=0\right\}=\mathrm{P}_{0}(\partial \mathrm{T})
$$

Next we develop an example where the above theory is applied.

THEOREM 20.2. Let $\Omega$ be an open polygonal domain in $\mathbb{R}^{2}$ triangulated by triangles $\mathrm{T}$. Let $\mathrm{k}$ be a positive integer, and put

$$
\mathbf{k}^{*}= \begin{cases}\mathbf{k} & \text { if } \mathbf{k} \text { is odd } \\ \mathbf{k}+1 & \text { if } \mathbf{k} \text { is even. }\end{cases}
$$

For each triangle $\mathrm{T} \in T_{\mathrm{h}}$ define the subspace $\mathrm{Y}_{\mathrm{T}} \subset \mathrm{H}^{\frac{1}{2}}(\partial \mathrm{T})$ by

$$
Y_{T}=P_{k}(\partial T)
$$

(With the definition of the space $\mathrm{L}_{\partial T_{\mathrm{h}}}^{(\mathrm{k})}$ given by (5.14), we have $\mathrm{M}_{\mathrm{h}}=\mathrm{L}_{\partial T_{\mathrm{h}}}^{(\mathrm{k})}$ ). Suppose that the space $\mathrm{Q}_{\mathrm{T}} \subset H(\mathrm{div}, \mathrm{T})$ is such that the following property is satisfied :

$$
\forall \mu \in \mathrm{D}_{\mathrm{k}} *(\partial \mathrm{T}), \exists \underline{\mathrm{q}} \in \mathrm{Q}_{\mathrm{t}} \text { s.t. } \underline{\mathrm{q}} \underline{\nu}_{-\mathrm{T}}=\mu \text { on } \partial \mathrm{T} \text {. }
$$

Then problem (20.10) has a unique solution.

To establish this theorem it clearly suffices to show that the local compatibility condition (20.13) is satisfied. A demonstration can be found in THOMAS (1976, lemma 4.1). We point out that the subspace of all $\psi \in \mathrm{P}_{\mathrm{k}}(\partial \mathrm{T})$ which satisfy $\int_{\partial \mathrm{T}} \psi \mu \mathrm{d} \sigma=0$ for each $\mu \in \mathrm{D}_{\mathrm{k}}(\partial \mathrm{T})$ with $\int_{\partial \mathrm{T}} \mu \mathrm{d} \sigma=0$ reduces to $\mathrm{P}_{0}(\partial \mathrm{T})$ only when $k$ is odd. Otherwise, when $k$ is even, it is a subspace of dimension 2.

We shall give error estimates using the norm $\|\cdot\|_{0, \Omega}$ on $\left(L_{2}(\Omega)\right)^{2}$ for

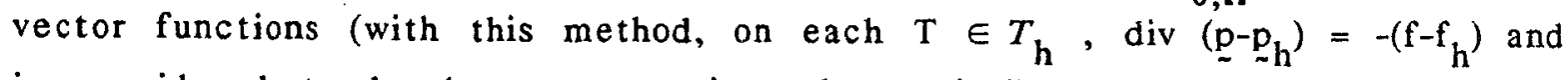
is considered to be known or estimated a priori) and the norm \|\|$\cdot \| i_{\frac{1}{2}, \partial T_{h}}$ given in (5.19) for the traces on $\partial T_{h}$ of functions in $H^{1}(\Omega)$. We suppose we have a uniformly regular family of triangulations, where the hypothesis of uniformness is made simply for ease of exposition. We note the existence of constants $c$ and $C_{k}$ independent of $h$ such that 
(20.16)

$$
\|\psi\|_{0, \partial T_{h}} \quad \leq \mathrm{ch}^{\frac{1}{2}}\|\psi\| \|_{\frac{1}{2}, \partial T_{h}}
$$

for all $\psi \in \mathrm{H}^{\frac{1}{2}}\left(\partial T_{\mathrm{h}}\right)$

and

$(20.17)$

$$
\|\| \psi_{h}\left\|_{\frac{1}{2}, \partial T_{h}} \leq C_{k} h^{-\frac{1}{2}}\right\| \psi_{h} \|_{0, \partial T_{h}}
$$$$
\text { for all } \psi_{h} \in L_{\partial T_{h}}^{(k)}
$$

THEOREM 20.3 - We suppose the hypotheses of Theorem 20.2 except here we fix for each $T \in T_{h}$

$$
Y_{T}=P_{k}(\partial T) \quad, \quad Q_{T}=D_{k}^{*(T)}
$$

Then there exists a constant $\mathrm{C}$ independent of h such that we have

$$
\left\|\underset{\sim}{p}-\underline{p}_{h}\right\|_{0, T_{h}}+\|\| \phi-\phi_{h}\|\|_{\frac{1}{2}, \partial T_{h}} \leq C h^{k}\left\{|\underline{p}|_{k, \Omega}+|u|_{k+1, \Omega}\right\}
$$

once the solution $(\mathrm{p}, \mathrm{u})$ of $(15.2)$ and of $(15.8)$ belongs to $\left(\mathrm{H}^{\mathrm{k}}(\Omega)\right)^{2} \times \mathrm{H}^{\mathrm{k}+1}(\Omega) ;(\phi$ is expressed as a function of $\mathrm{u}$ in (20.6)).

Proof. To apply the general theory developed in Chapter 3 we begin by

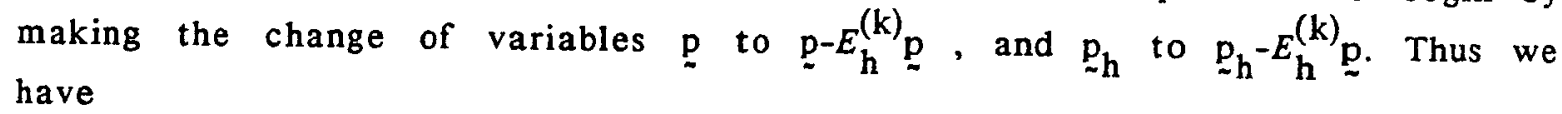

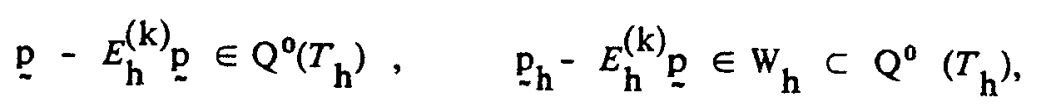

where $E_{\mathrm{h}}^{(\mathrm{k})} \underline{\mathrm{p}}$ is the equilibrium interpolant of order $\mathrm{k}$ described in Section 6 . The continuity properties and $W_{h}$-ellipticity with constants independent of $h$ relative to the norm $\|\cdot\|_{0, \Omega}$ are obvious. Further, we can establish the existence of constants $B$ and $\beta_{0}>0$, independent of $h$, such that

$$
\mathrm{b}(\mathrm{q}, \psi) \leq \mathrm{B}\|\underline{\mathrm{q}}\|_{0, \Omega}\|\| \psi\|\|_{\frac{1}{2}, \partial T_{\mathrm{h}}}
$$

and

$$
\begin{aligned}
& \text { for all } \underline{q} \in \mathrm{Q}^{0}\left(T_{\mathrm{h}}\right) \\
& \text { and all } \psi \in \mathrm{L}\left(\partial T_{\mathrm{h}}\right)
\end{aligned}
$$

$$
\sup _{q_{h} \in W_{h}} \frac{b\left(\underline{q}_{h}, \psi_{h}\right)}{\left\|q_{h}\right\|_{0, \Omega}} \geq \beta_{0} \quad\left[\psi_{h}\right]_{\frac{1}{2}, \partial T_{h}} \quad \text { for all } \psi_{h} \in M_{h}
$$

where the semi norm $[\cdot]_{\frac{1}{2}, \partial T_{h}}$ is defined by

$$
[\psi]_{\frac{1}{2}, \partial T_{h}}=\left\{\sum_{T \in T_{h}}\left\|\psi_{T}-\frac{1}{\operatorname{mes} \partial T}\left(\int_{\partial T} \psi_{T} d \sigma\right)\right\|_{\frac{1}{2}, \partial T}^{2}\right\}^{\frac{1}{2}}
$$


Using the Poincaré-Friedrichs inequality we can show that $[\cdot]_{\frac{1}{2}, \partial T_{h}}$ is a norm on $M_{0 h}$ equivalent (uniformly in $h$ ) to the norm \|\|$\cdot\|\|_{\frac{1}{2}, \partial T_{h}}$. Thus there exists $\beta>0$, independent of $h$, such that

$$
\sup _{\underline{q}_{h} \in W_{h}} \frac{b\left(\underline{q}_{h}, \psi_{h}\right)}{\left\|\underline{q}_{h}\right\|_{0}, \Omega} \geq \beta\left\|\psi_{h}\right\| \|_{\frac{1}{2}, \partial T_{h}} \quad \text { for all } \psi_{h} \in M_{0 h} \text {, }
$$

The theorem then follows easily.

Remark 20.3 . When the integer $k$ is even, we can obtain the same result while taking the space $Q_{T}$ to be the space generated by $D_{k}(T)$ together with an appropriately chosen element of $D_{k+1}(T)$. For details and other examples cf. THOMAS (1976).

The dual hybrid finite element method corresponding to the choice of subspaces $M_{h}$ and $W_{h}$ associated with (20.18) by (20.8) and (20.9) is convergent for each integer $k \geq 1$ in the following sense : (with no regularity hypothesis for the solution, one can show that):

$$
\lim _{h \rightarrow 0}\left\|\underline{p}-\underline{p}_{h}\right\|_{0, \Omega}=0
$$

The principle of the dual hybrid finite element methods given above for a second order elliptic problem corresponds to that developed by specialists in structural mechanics and termed assumed stress hybrid finite element model. We cite first of all the work of PIAN (1964), (1971), (1972), (1983) ; cf. also PIAN \& TONG (1969), (1986), TONG (1983), and the recent articles of PIAN and his collaborators where can be found a treatment of nonaffine-equivalent finite elements. Other examples are given in ATLURI (1971), ATLURI, TONG \& MURAKAWA (1983), PUNCH \& ATLURI (1984), SPILKER \& MUNIR (1980a), (1980b) and WOLF (1972a), (1972b) amoung many other publications on the subject. For a mathematical analysis of such methods we refer to the work of BREZZI (1975), (1977), of KIKUCHI (1973) and of QUARTERONI (1979).

Remark 20.4. The construction of the examples given above followed this strategy : given a space $M_{h}$, find a space $W_{h}$ large enough for the compatibility condition to be satisfied. Undê reasonable hypotheses we obtain asympotic orders of optimal error (relative to the given $W_{h}$ ). The construction of examples proposed by engineers of ten follows another strategy : given two 
spaces $W_{h}$ and $M_{h}^{*}$, the choice of spaces being consistent with the physics of the problem, we note the existence of "mechanisms" (... the homogeneous problem does not have the trivial solution as unique solution). One then tries to eliminate these mechanisms, that is to reduce $M_{h}^{*}$ to a space $M_{h}$ small enough for the compatibility condition with $W_{h}$ to be satisfied. This technique does not lead to satisfactory asymptotic orders of convergence.

\section{1 - HYBRIDIZATION OF EQUILIBRIUM METHODS}

The dual hybrid finite element method just constructed yields - when the compatibility hypothesis (18.12) is satisfied - an approximation $\mathfrak{\sim}_{h}$ of $\underset{\sim}{p}$ satisfying div ${\underset{\sim}{h}}_{h}+f_{h}=0$ in the interior of each finite element $T$; however, the reciprocity of the normal traces on the interfaces is not realized pointwise along the entire interface. In particular, the solution ${\underset{\sim}{h}}_{h}$ is not in $H($ div; $\Omega$ ).

A variant introduced by FRAEIJS DE VEUBEKE (1965) yields an approximated solution $\underset{\sim}{p_{h}} \in H($ div; $\Omega)$ satisfying div $\underset{\sim}{p_{h}}+f_{h}=0$ in $\Omega$. The mathematical analysis can be found in THOMAS (1977, Chapter VIII), cf. also RAVIART \& THOMAS (1979). These results are completed by a postprocessing technique developed in ARNOLD \& BREZZI (1985).

We thus reconsider the approximation of the solution $(p, \phi)$ of the model problem with the dual hybrid formulation (20.2) - (20.5). On the one hand, for each $\mathrm{T} \in T_{\mathrm{h}}$, we take a finite dimensional subspace $\mathrm{Q}_{\mathrm{T}} \subset H(\mathrm{div} ; \mathrm{T})$ and as before, cf. (20.7), (20.8), define the spaces

$$
\mathrm{w}_{\mathrm{h}}^{\mathrm{f}}=\left\{\underline{\mathrm{q}} \in H\left(\operatorname{div} ; T_{\mathrm{h}}\right) ; \forall \mathrm{T} \in T_{\mathrm{h}}, \underline{-q}_{\mathrm{T}} \in \mathrm{Q}_{\mathrm{T}}\right\} \cap \mathrm{Q}^{\mathrm{f}_{\mathrm{h}}}\left(T_{\mathrm{h}}\right)
$$

and

$$
\mathrm{w}_{\mathrm{h}}=\mathrm{w}_{\mathrm{h}}^{0}=\left\{\mathrm{q} \in H\left(\operatorname{div} ; T_{\mathrm{h}}\right) ; \forall \mathrm{T} \in T_{\mathrm{h}}, \underline{\mathrm{q}}_{\left.\right|_{\mathrm{T}}} \in \mathrm{Q}_{\mathrm{T}}\right\} \cap \mathrm{Q}^{0}\left(T_{\mathrm{h}}\right) .
$$

On the other hand, for each $T \in T_{h}$, we put

$$
\mathbf{Y}_{\mathbf{T}}=\left\{\psi \in \mathrm{L}^{2}(\partial \mathrm{T}) ; \exists \underset{\sim}{\mathrm{q}} \in \mathrm{Q}_{\mathrm{T}} \text { s.t. } \underline{\mathrm{q}}_{\sim_{-} \mathrm{T}}=\psi\right\}
$$

and define

$$
\begin{array}{r}
\mathrm{M}_{\mathrm{h}}=\left\{\psi=\left({ }_{\mathrm{T}}\right)_{\mathrm{T} \in T_{\mathrm{h}}} \in \mathrm{L}^{2}\left(\partial T_{\mathrm{h}}\right) ; \forall \mathrm{T} \in T_{\mathrm{h}}, \psi_{\mathrm{T}} \in \mathrm{Y}_{\mathrm{T}}\right. \text { and } \\
\left.\forall \mathrm{T}^{\prime}=\mathrm{T}_{1} \cap \mathrm{T}_{2} \text { with } \mathrm{T}_{1}, \mathrm{~T}_{2} \in T_{\mathrm{h}}, \psi_{\mathrm{T}_{1}}=\psi_{\mathrm{T}_{2}} \text { on } \mathrm{T}^{\prime}\right\}
\end{array}
$$


We seek $\left({\underset{\sim}{h}}_{h}, \phi_{h}\right)$ satisfying (cf. $(20.10)$ )

$$
\begin{cases}\left(\sim_{h}, \phi_{h}\right) \in W_{h}^{f} \times M_{h}, & \\ a\left(\underline{p}_{h}, q_{h}\right)+b\left(\underline{q}_{h}, \phi_{h}\right)=0 & \text { for all } \underline{q}_{h} \in W_{h}, \\ b\left(\underline{p}_{h}, \psi_{h}\right)+d\left(\phi_{h}, \psi_{h}\right)=-\sum_{T \in T} \int_{\partial T \cap \Gamma} g \psi_{h T} d \sigma . & \text { for all } \psi_{h} \in M_{h} .\end{cases}
$$

Remark 21.1 . Such an approximation method is not a dual hybrid approximation in the sense of the preceeding section because the spaces $Y_{T}$ are not subspaces of $H^{\frac{1}{2}}(\partial T)$, and thus the space $M_{h}$ of multipliers is not a subspace of the space $L\left(\partial T_{h}\right)$ defined in (18.4) or (18.5). Whereas examples of finite dimensional subspaces of $L^{2}(\partial T)$ and of $H^{-\frac{1}{2}}(\partial T)$ are identical, the examples of finite dimensional subspaces of $\mathrm{L}^{2}(\partial T)$ and of $\mathrm{H}^{\frac{1}{2}}(\partial \mathrm{T})$ are fundamentally different : the functions in $\mathrm{H}^{\frac{1}{2}}(\partial \mathrm{T})$ which are locally continuous are globally continuous on $\partial \mathrm{T}$.

It is clear that for each choice of spaces $\mathrm{Q}_{\mathrm{T}} \subset H(\operatorname{div} ; \mathrm{T})$ with $\mathrm{Y}_{\mathrm{T}}$ associated to $Q_{T}$ by (21.3), problem $(21.5)$ has a unique solution $\left(\underline{p}_{h}, \phi\right)$.

Suppose that the coefficient $t>0$ is constant on each face $T$ 'contained in the boundary $\Gamma$ of $\Omega$ - (this hypothesis is made solely for simplicity of exposition). Then the elimination of the multiplier $\phi_{h}$ in (21.5) yields a characterization of $\underset{\sim}{p_{h}}$ as the solution of the variational problem

$$
\left\{\begin{array}{l}
\stackrel{p}{h}_{h} \in V_{h}^{f}, \\
\sum_{i j=1}^{n} \int_{T} A_{i j} p_{h j} q_{h i} d x+\int_{\Gamma} \frac{1}{t}\left(\underline{\sim}_{h} \cdot \underset{\sim}{\nu}\right)\left({\underset{\sim}{h}}_{h} \cdot \underset{\sim}{\nu}\right) d \sigma=\int_{\Gamma} \frac{1}{t} g \underline{q}_{h} \cdot \underset{\sim}{\nu} d \sigma \\
\quad \text { for all } \underline{q}_{h} \in V_{h},
\end{array}\right.
$$


The approximation ${\underset{\sim}{p}}_{h}$ of $\underline{p}$ thus obtained is an equilibrium approximation (cf. Section 2); i.e. $\stackrel{\sim}{h}_{h}$ is a vector function in $H($ div; $\Omega)$ that satisfies the equilibrium equation

$$
\operatorname{div} \underset{\sim h}{p_{h}}+f_{h}=0 \quad \text { in } \Omega
$$

Conversely, to an equilibrium formulation (21.6) - (21.8) can be associated a hybrid method defined by (21.1) - (21.5). Contrary to what one might suspect at first glance, it is simpler to solve numerically (21.5) than (21.6). The numerical solution of (21.5) can be carried out by first eliminating the principal variable $\stackrel{\sim}{\mathrm{p}}_{\mathrm{h}}$. (This elimination is performed at the level of the n-simplex $T$ ). Then to calculate $\phi_{h}$, one must solve a linear system of the same structure as the one which results from a nonconforming finite element method, cf., in particular, FRAEIJS DE VEUBEKE (1974a).

Remark 21.2 . When the given function $f$ is trivial, or sufficiently simple that one can numerically reduce the problem to the case $f=0$, to solve (19.6) (equilibrium formulation) it suffices to know how to construct a basis for $V_{h}$. In dimension $\mathrm{n}=2$, using stream functions we can treat directly problem (21.6), cf. HASLINGER \& HLAVACEK (1975), (1976a), (1976b), HLAVACEK (1980), and HLAVACEK \& KRIZEK (1984). We have not chosen this presentation because it is not easily generalized in dimension $n=3$ to the case where $f$ is arbitrary and the boundary condition is a Robin's condition.

When $T_{h}$ is a triangulation of $\bar{\Omega}$ by $n$-simplexes $T$, if we take for spaces $Q_{T}$ the spaces $D_{k}(T)$, where $k$ is a given integer, $k \geq 1$, the described method is to take for spaces $Y_{T}$ the spaces $D_{k}(\partial T)$. Thus here we have

$$
\mathrm{w}_{\mathrm{h}}^{\mathrm{f}}=\left\{\underline{\mathrm{q}} \in H\left(\operatorname{div} ; T_{\mathrm{h}}\right) ; \forall \mathrm{T} \in T_{\mathrm{h}}, \underline{-q}_{\mathrm{T}} \in \mathrm{D}_{\mathrm{k}}(\mathrm{T})\right\} \cap \quad \mathrm{Q}^{\mathrm{f}_{\mathrm{h}}}\left(T_{\mathrm{h}}\right)
$$

and

$$
\mathrm{W}_{\mathrm{h}}=\mathrm{W}_{\mathrm{h}}^{0}=\left\{\underline{\mathrm{q}} \in H\left(\operatorname{div} ; T_{\mathrm{h}}\right) ; \forall \mathrm{T} \in T_{\mathrm{h}}, \underline{-}_{\mathrm{T}} \in\left(\mathrm{P}_{\mathrm{k}-1}(\mathrm{~T})\right)^{\mathrm{n}}\right\} \cap \mathrm{Q}^{0}\left(T_{\mathrm{h}}\right)
$$

where for the characterization of $W_{h}$ we have used the fact that each vector function in $D_{k}$ that is divergence free belongs in fact to $\left(P_{k-1}(T)\right)^{n}$. The associated space $V_{h}$ is then none other than 
(21.12)

$$
\mathrm{V}_{\mathrm{h}}=\left\{\underline{\mathrm{q}} \in H(\operatorname{div} ; \Omega) ; \operatorname{div} \underline{\mathrm{q}}=0 \text { on } \Omega \text { and s.t. } \forall \mathrm{T} \in T_{\mathrm{h}}, \mathrm{q}_{-\mathrm{T}} \in\left(\mathrm{P}_{\mathrm{k}-1}\right)^{\mathrm{n}}\right\},
$$

i.e. with the notation of (6.17)

$$
V_{h}=E_{h}^{(k)} \cap\left\{\underline{q} \in\left(L^{2}(\Omega)\right)^{n} ; \operatorname{div} \underline{q}=0 \text { on } \Omega\right\}
$$

For simplicity of exposition, to give error estimates we suppose that the family of triangulations $T_{h}$ is uniformly regular. With $Q_{T}=D_{k}(T)$ and $Y_{T}=D_{k}(\partial T)$ for each $T \in T_{h}$, there is no difficulty in showing that there is a constant $\beta_{0}$ $>0$, independent of $h$, such that

$$
\sup _{\mathrm{q}_{\mathrm{h}} \in \mathrm{W}_{\mathrm{h}}} \frac{\mathrm{b}\left(\mathrm{q}_{\mathrm{h}}, \psi_{\mathrm{h}}\right)}{\left\|\mathrm{q}_{\mathrm{h}}\right\|_{0, \Omega}} \geq \beta_{0} \mathrm{~h}^{-\frac{1}{2}}\left[\psi_{\mathrm{h}}\right]_{0, \partial T_{\mathrm{h}}} \quad \text { for all } \psi_{\mathrm{h}} \in \mathrm{M}_{\mathrm{h}},
$$

where the semi-norm $[\cdot]_{0, \partial T_{\mathrm{h}}}$ is defined by

$$
[\psi]_{0, \partial T_{\mathrm{h}}}=\left\{\sum_{\mathrm{T} \in T_{\mathrm{h}}}\left\|\psi_{\mathrm{T}}-\frac{1}{\operatorname{mes} \partial \mathrm{T}}\left(\int_{\partial \mathrm{T}} \psi_{\mathrm{T}} \mathrm{d} \sigma\right)\right\|_{0, \partial \mathrm{T}}^{2}\right\}^{\frac{1}{2}} .
$$

Thus we have

THEOREM 21.1. Suppose that, for each $\mathrm{T} \in T_{\mathrm{h}}$,

$$
Q_{T}=D_{k}(T), \quad Y_{T}=D_{k}(\partial T)
$$

Then there exists a constant $\mathrm{C}$, independent of $\mathrm{h}$, such that if $\left(\mathrm{p}_{\mathrm{h}}, \phi_{\mathrm{h}}\right)$ is the solution of (21.5), then

$$
\left\|\underline{p}-{\underset{\sim}{h}}_{h}\right\|_{0, \partial T_{h}}+h^{-\frac{1}{2}}\left\|\phi-\phi_{h}\right\|_{0, \partial T_{h}} \leq C h{ }^{k}\left\{|\underline{p}|_{k, \Omega}+|u|_{k+1, \Omega}\right\},
$$

provided, of course, that the solution $(\underset{\sim}{\mathrm{p}}, \mathrm{u})$ of $(15.2)$ and of $(15.8)$ belongs to $\left(\mathrm{H}^{\mathrm{k}}(\Omega)\right)^{\mathrm{n}} \times$ $\mathrm{H}^{\mathrm{k}-1}(\Omega) . \quad(\phi$ is expressed as a function of $\mathrm{u}$ in (20.6) ). 
Once the solution $\left(\underline{p}_{h}, \phi_{h}\right)$ of problem (21.5), with the spaces $Q_{T}$ and $Y_{T}$ as given in (21.13), has been obtained, we can easily calculate the function $u_{h} \in$ $L^{2}(\Omega)$ whose restriction to each $T \in T_{h}$ is the polynomial of degree at most $k-1$ defined by

$$
\begin{array}{r}
\int_{T} u_{h} \operatorname{div} q d x=-\sum_{i, j=1}^{n} \int_{T} A_{i j} p_{h j} q_{i} d x+\int_{\partial T} \phi_{h T} \underline{q} \cdot \nu_{T} d \sigma \\
\text { for each } \underline{q} \in D_{k}(T) .
\end{array}
$$

Using that $\left(\mathfrak{\sim}_{h}, \phi_{h}\right)$ satisfies $(21.5)$ we remark that this function $u_{h}$ satisfies

$$
\begin{aligned}
& \int_{\Omega} u_{h} \operatorname{div}{\underset{\sim}{h}}_{h} d x=-\sum_{i, j=1}^{n} \int_{\Omega} A_{i j} p_{h j} a_{h i} d x+\int_{\Gamma} \frac{1}{t}(g-\underset{h}{p} \cdot \underset{\sim}{\nu}) \underset{-h}{q} \cdot \nu d \sigma \\
& \text { for each }{ }_{{ }_{h}} \in H(\operatorname{div} ; \Omega) \text { such that }{ }_{-h}^{\mathrm{q}_{\mathrm{h}}} \in \mathrm{D}_{\mathrm{k}}(\mathrm{T}) \text { for each } \mathrm{T} \in T_{\mathrm{h}} \text {. }
\end{aligned}
$$

The pair $\left(\mathrm{p}_{h}, \mathrm{u}_{h}\right)$ is the unique solution of problem (15.14) (dual mixed formulation) obtained with (15.12) and (15.13). Thus we know $u_{h}$ is a approximation of $u$ of order $k$ relative to the norm $\|\cdot\|_{0, \Omega}$.

Now for each $T \in T_{h}$, we have both the approximation $\phi_{h T} \in D_{k}(\partial T)$ of $\phi_{T}=u_{1 \partial T}$ and the approximation $u_{h l} \in P_{k-1}(T)$ of $u_{1 T}$. These two approximations provide complementary information about $\mathrm{u}_{\mathrm{T}}$ and it is reasonable to try to use all of this information to obtain a better approximation of $u_{T}$. We take an example in dimension $n=2$ when $k$ is add. The relations

and

$$
\int_{\partial T}\left(\mathrm{u}_{\mathrm{h}}^{*}-\phi_{\mathrm{hT}}\right) \psi \mathrm{d} \sigma=0 \quad \text { for all } \psi \in \mathrm{D}_{\mathrm{k}}(\partial \mathrm{T})
$$

$$
\int_{T}\left(u_{h}^{*}-u_{h}\right) v d x=0 \quad \text { for all } v \in P_{k-3}(T) \text { (if } k \geq 3 \text { ) }
$$

define a unique function $u_{h}^{*} \in L^{2}(\Omega)$ whose restriction to each triangle $T \in T_{h}$ is a polynomial of degree at most $k$. Under the standard regularity hypotheses, one can show (ARNOLD \& BREZZI (1985, Theorem 2.2)) the order of approximation is thus improved :

$$
\left\|u-u_{h}^{*}\right\|_{0, \Omega}=O\left(h^{k+1}\right)
$$




\section{CHAPTER VI}

\section{EXTENSIONS AND VARIATIONS}

\section{2 - OTHER EXAMPLES OF MIXED AND HYBRID METHODS}

The mixed and hybrid finite element methods given in the preceding sections were developed almost exclusively for second order elliptic problems. Formulations of such methods for fourth order elliptic problems were noted. The mathematical framework constructed for the numerical analysis of these methods for approximating the solutions of partial differential aquations apparently ignores the physical origin of the model. We have not even sought to exploit the supplementary properties that might result from the fact in most cases we are concerned with the solutions of contrained minimization problems. This does not however imply that the elaboration of such approximation techniques can be successfully carried out without taking into account the physical properties of the underlying problem. There is no lack of examples in computational mechanics in the recent scientific literature. We give here only a brief overview.

Stokes' problem is a fundamental problem of fluid mechanics. The primary unknown $u$ is the velocity vector for the fluid displacement. It can be characterized as being the solution of a minimization problem in a subspace of the space

$$
\mathrm{V}=\left\{\underline{\mathrm{v}}=\left(\mathrm{v}_{\mathrm{i}}\right) ; \mathrm{v}_{\mathrm{i}} \in \mathrm{H}^{1}(\Omega), 1 \leq i \leq n \text {, s.t. } \operatorname{div} \underline{\mathrm{v}}=0\right\}
$$

The Lagrangian multiplier associated with the constraint that the velocity field be divergence free is identifield with a pressure. The approximation of the solution of this problem has been and will continue to be the object of many publications. The state of the art in this domain can be found in the book of GIRAULT \& RAVIART (1986) wich has recently appeared, and it does not seem opportune to duplicate here the bibliography of this work.

The system of linear elasticity constitutes another priviledged domain for the application of the theory of mixed and hybrid methods. The variational 
formulation of the particle displacement being taken as primal formulation, the dual formulation called the equilibrium formulation, is a variational formulation of the stress equation. This equilibrium formulation is a minimization principle in a subspace of the space

$$
S=\left\{\underset{\approx}{\sigma}=\left(\sigma_{i j}\right) ; \sigma_{i j} \in L^{2}(\Omega), \sigma_{i j}=\sigma_{j i}, 1 \leq i, j \leq n, \operatorname{div} \underset{\approx}{\sigma}=f\right\} .
$$

With some simplifying restrictions concerning the data and geometry of the problem under consideration, it is possible to use Airy functions to reduce the study of the equilibrium formulation to that of the two-dimensional biharmonic problem. The degree of sophistication necessary for constructing equilibrium finite element models is more easily understood with this analogy. Examples can be found in JOHNSON \& MERCIER (1978), (1979), KRIZEK (1982), (1983), and KRIZEK \& NEITTAAMAKI (1986). Analyses of mixed and hybrid formulations for the system of elasticity are proposed in AMARA \& THOMAS (1978), (1979), ARNOLD, BREZZI, \& DOUGLAS (1985), ARNOLD, DOUGLAS, \& GUPTA (1984), PITKARANTA \& STENBERG (1983), and STENBERG (1986),(1987a), (1987b). The principal source of difficulty in the approximation of the stress formulation comes from the necessity of respecting, rigorously or not, the symmetry relations that should be satisfied by the stresses. The article of ARNOLD \& FALK (1987) proposes a new formulation with one obtains distinct approximations of $\sigma_{\mathrm{ij}}$ and $\sigma_{\mathrm{ji}}$

Other recent articles concerning the numerical treatment of problems of solid mechanics with mixed and hybrid methods are referenced in the bibliography. While some of these articles may appear to lack a certain degree of mathematical rigor, experience has shown that the examples described therein can not, nevertheless, be neglected.

Another domain where quite a few models using mixed or hybrid finite elements have been constructed is the subdomain of fluid mechanics dealing with fluid flow in porous media and in particular petroleum reservoir engineering. These reservoir models are based on nonlinear equations too complex to be described here in a few lines. We refer the interested reader to the book of CHAVENT \& JAFFRE (1986), where in Chapter V some models directly inspired by the mixed methods developed above may be found. Other models are described and analyzed in CHAVENT, COHEN, DUPUY, JAFFRE \& RIBERA (1984), DOUGLAS (1984), DOUGLAS, EWING, \& WHEELER (1983), DOUGLAS \& RODERTS (1983), DARLOW, EWING $\hat{\&}$ WHEELEK (1984), EYMARD, GALLOUET, \& JOLY (1987), JAFFRE \& ROBERTS (1985), and WHEELER \& GONZALES (1984).

We conclude this section by mentioning that mixed finite elements have also been used for the equations of electromagnetism : NEDELEC (1980), BENDALI (1984), BOSSAVIT (1987). 
The methods of Lagrangian multipliers that have been presented here concern the approximation of elliptic problems. These methods have extended to the study of eigenvalue problems; cf. CANUTO (1978), MERCIER, OSBORN, RAPPAZ, \& RAVIART (1981). They have also been extended to the study of problems of evolution : parabolic problems as in JOHNSON \& THOMEE (1981) and in THOMEE (1984), hyperbolic problems as in CANUTO (1981), in DOUGLAS \& GUPTA (1986), and in QUARTERONI (1980b).

Certain mixed methods have been successful only because of the use of reduced numerical integration. Some examples can be found in JOHNSON \& PITKARANTA (1982), MALKUS \& HUGUES (1978), NOOR \& PETERS (1983), SHIMODAIRA (1985). Other examples of reduced integration are proposed in RAVIART \& THOMAS (1977b) to obtain the interpretation of certain primal hybrid methods as nonconforming methods.

In the preceding studies the approximation of elliptic problems of constrained minimization has been approached exclusively with the technique of dualization of the constraints using Lagrangian multipliers. The technique of penalization of the constraints constitutes an alternative way of attacking these minimization problems. These techniques can fortunately be combined. The resulting technique is called the augmented Lagrangian technique for which we refer the reader to the book of FORTIN \& GLOWINSKI (1983). Even though the penalization technique was not initially retained in the mixed and hybrid formulations of the problem, it can nonetheless be useful for the resolution of the linear system resulting from the mixed hybrid formulation; cf. BERCOVIER (1978), FELIPPA (1986) ; cf. also Section 17.

Other finite element methods such as nodal methods can be interpreted as being mixed or hybrid methods; cf. HENNART (1985), (1986), and HENNART, JAFFRE, \& ROBERTS (1986). The so called Trefftz methods can also be understood as being variantes of the dual mixed hybrid formulations; cf. JIROUSEK \& LAN GUEZ (1986) and ZIELINSKI \& ZIENKIEWICZ (1985).

As an extension of the classical abstract theory of Ritz-Galerkin, with the development of the theory of internal approximation of variational problems which has been seen to be fundamental for the analysis of "standard" finite element methods, the abstract theory of Babuska-Brezzi for the approximation of problems with Lagrangian multipliers has become popular because of its applications to the analysis of mixed and hybrid finite element methods. Other 
applications of the Babuska-Brezzi theory have been proposed more recently ; cf; for example the analysis of spectral methods proposed in the articles of BERNARDI, CANUTO, \& MADAY (1987), and of BERNARDI, MADAY, \& METIVET (1987) or the analysis of modal decomposition methods in the articles of STOLARSKI \& BELYTSCHKO (1986a), (1986b).

Domain decomposition methods for the solution of partial differential equations are concurrently enjoying a resurgence in popularity for their use in constructing algorithms well adapted to use on multiprocessing machines. The use of mixed and hybrid formulations seem adequate for this type of method; cf. WHEELER \& GLOWINSKI (1987) for a first example in this direction. 
ADAMS, R.A. (1975) : "Sobolev Spaces " Academic Press, London.

AMARA, M. : THOMAS, J.M. (1978) : Approximation por eléments finis equilibre du systeme de l'elasticité linéaire, C.R.A.s. Serie A 286, Paris, 1147-1150.

AMARA, M. ; THOMAS, J.-M. (1979) : Equilibrium finite elements for the linear elastic problem, Numer. Math. $33,367-383$.

ARNOLD, D.N. ; BREZZI, F. (1985) : Mixed and nonconforming finite element methods : implementation, postprocessing and error estimates, M2AN $19,7-32$.

ARNOLD, D.N. ; BREZZI, F. ; DOUGLAS, J.Jr. (1985) : Peers: a new mixed finite element for plane elasticity, Japan J. Appl. Math. $1,347-367$.

ARNOLD, D.N. ; DOUGLAS, J.Jr. ; GUPTA, C.P. (1984): A family of higher order mixed finite element methods for plane elasticity, Numer. Math. 45, 1-22.

ARNOLD, D.N. ; FALK, R. (1987) : A new mixed formulation for elasticity, to appear.

ATLURI, S.N. (1971) : A new assumed stress hybrid finite element model for solid continua, A.I.A.A. J. $9,1647-1649$.

ATLURI, S.N. ; TONG, P. ; MURAKAWA, H. (1983) : Recent studies in hybrid and mixed finite element methods in mechanics. in "Hybrid and Mixed Finite Element Methods" (S.N. Atluri. R.H. Gallagher, \& O.C. Zienkiewicz, Eds.). pp.51-71, John Wiley \& Sons, Chichester.

BABUSKA, I. (1971): Error bounds for finite element method. Numer. Math. $16,322-333$.

BABUSKA, I. (1973): The finite element method with Lagrange multipliers, Numer. Math., $20,179-192$.

BABUSKA, I. ; AZIZ. A.K. (1972) : Survey lectures on the mathematical foundations of the finite element method. in "The Mathematical Foundations of the Finite Element Method with Applications to Partial Differential Equations "( A.K. Aziz, Ed.), pp.3-359, Academic Press, New York.

BABUSKA, I. ; MILLER, A. (1984): The postprocessing approach in the finite element method. Part 1 : Calculation of displacements, stresses and other higher derivatives of the displacements, Internat. J. Numer. Meths. Engrg. 20, 1085-1109.

BABUSKA; I. ; ODEN, J.T. ; LEE, J.K. (1977) : Mixed-hybrid finite element approximations of second-order elliptic boundaryvalue problem -Part 1. Comput. Methods Appl. Mech. Engrg. 11. 175-206. 
BABUSKA, I. ; ODEN, J.T. ; LEE, J.K. (1978) : Mixed-hybrid finite element approximstions of second-order eiliptis boundaryvalue problem-Part 2: Weak-hybrid methods, Comput. Methods Appl. Mech. Engrg. $141,1-22$.

BABUSKA, I. ; OSBORN, J.E. (1983): Generalized finite element methods: their performance and their relation to mixed methods, SIAM J. Numer. Anal. $20,510-536$.

BABUSKA, I. ; OSBORN, J.E. ; PITKARANTA, J. (1980) : Analysis of mixed methods using mesh dependent norms, Math. Comput. 35. $1039-1062$.

BARLOW, J. (1986); A different view of the assumed stress hybrid method, Internat. J. Numer. Meths. Engrg. 22 , 11-16.

BENDALI, A. (1984a): Numerical analysis of the exterior boundary value problem for the time-hormonic Maxwell equations by a boundary finite element method-Part 1: The continuous problem. Math. Comput. $43,29-46$.

BENDALI, A. (1984b) : Numerical analysis of the exterior boundary value problem for the time-harmonic Maxwell equations by a boundary finite element method- Part 2: The discrete problem. Math. Comput. 43, 47-68.

BERCOVIER, M. (1978) : Pertubation of mixed variational problems. Applicotion to mixed finite element methods, R.A.I.R.O. Anal. Numer. $12,211-236$.

BERNARDI, C. (1986) : Contributions a l'Analyse Numerique de Problemes Non Linéaires, These d'Etat, Université Pierre et Marie Curie, Paris.

BERNARDI, C. ; CANUTO, C. ; MADAY, Y. (1987) : Generalized inf-sup condition for Chebyshev approximation of the Novier-Stokes equations, submitted to SIAM J. Numer. Anal.

BERNARDI, C. ; MADAY, Y. ; METIVET, B. (1987) : Calcul de la pression dans la résolution spectrale du probleme de Stokes, La Recherche Aerospatiale.

BOSSAVIT, A. (1987) : Mixed finite elements and the complex of Whitney forms, MAFELAP 87, London 1987, in " The Mathematics of Finite Elements and Applications ". Academic Press, New York, to appear.

BRAMBLE, J.H. (1981) : The Lagrange multiplier method for Dirichlet's problem, Math. Comput. $37,1-11$.

BRAMBLE, J.H. ; FALK, R.S. (1983) : Two mixed finite element methods for the simply supported plate Problem, R.A.I.R.O. Anal. Numér. $17,337-384$.

BRAMBLE, J.H. : FALK, R.S. (1985) : A mixed-Lagrange multiplier finite element method for the polyharmonic equation. M2AN $19.519-557$.

BREZZI, F. (1974a) : Theorèmes d'existence, diunicité et d'approximation numerique pour des problemes de point-selle. C.R.A.S. Serie A 278 , 839-842. 
BREZZI, F. (1974b) : On the existence, uniqueness and oppro$x$ imation of saddle-point problems arising from Lagrangian multipliers, R.A.I.R.O., Anal. Numer., 8, R2, 129-151.

BREZZI, F. (1974c) : Sur une méthode hybride pour l'approximation du probleme de la torsion d'une barre elastique, Ist. Lombardo (Rend. Sc.) A $108,274-300$.

BREZZI, F. (1975) : Sur la méthode des eléments finis hybrides pour le probleme biharmonique, Numer. Math. $24,103-131$.

BREZZ1, F. (1977) : Hybrid method for fourth order elliptic equations, in "Mathematical Aspects of Finite Element Methods " (I. Galligani \& E. Magenes, Eds.). Pp.35-46, Lecture Notes in Mathematics. Vol. 606 , Springer-Verlag, Berlin.

BREZZI, F. (1979) : Non-standard finite elements for fourth order elliptic problems, in "Energy Methods in Finite Element Analysis" (R. Glowinsky, E.Y. Rodin, \& O.C. Zienkiewicz, Eds.), Pp.193-211, John Wiley \& Sons, Chichester.

BREZZI, F. ; DOUGLAS, J.Jr. ; DURAN, R. ; FORTIN, M. (1987) Mixed finite elements for second order elliptic problems in three variables, to appear in Numer. Math.

BREZZI, F. ; DOUGLAS, J.Jr. ; FORTIN, M. ; MARINI, L.D. (1987): Efficient rectangular mixed finite elements in two and three variables, to appear in Numer. Math.

BREZZI, F. ; DOUGLAS, J.Jr. ; MARINI, L.D. (1985) : Two families of mixed finite elements for second order elliptic problems, Numer. Math. 47, 217-235.

BREZZI, F. ; FORTIN, M. (1986) : Numerical Approximation of Mindlin Reissner plates, Math Comp. 47, 151-158.

BREZZI, F. ; MARINI, L.D. (1975) : On the numerical solution of plate bending problems by hybrid methods, R.A.I.R.O. Anal. Numér. 9 , No $19,5-50$.

BREZZI, F. ; RAVIART, P.A. (1978): Mixed finite element methods for 4th order elliptic equations, in "Topics in Numerical Analysis III "( J. Miller, Ed.), pp.35-56, Acodemic Press, New York.

BROWN, D. C. (1982) : Alternating-direction iterative schemes for mixed finite element methods for second order elliptic problems, Thesis. The University of Chicago.

CANUTO, C. (1978) : Eigenvalue approximations by mixed methods, R.A.I.R.O., Anal. Numér., $12,27-50$.

CANUTO, C. (1981) : A hybrid finite element method to compute the free vibration frequencies of a clamped plate. R.A.I.R.O., Anal. Numer., $15,101-118$.

CAREY, G.F. ; CHOW, S.S. ; SEAGER, M.K. (1985) : Approximate boundary-flux calculations, Comput. Methods Appl. Mech. Engrg. $50,107-120$.

CHAVENT, G. ; COHEN, G. ; DUPUY. M. ; JAFFRE, J. ; RIBERA, I. 
(1984) : Simulation of two dimensional waterflooding using mixed finite elements, SPE J. $24,382-390$.

CHAVENT, G. ; JAFFRE J. (1986) : "Mathematical Models and Finite Elements for Reservoir Simulation" Studies in Mathematics and its Applications Vol. 17. North-Hol land, Amsterdam.

CIARLET, P.G. (1974) : Conforming and nonconforming finite element methods for solving the plate problem, in "Numerical Solution of Differential Equations "( G.A. Watson. Ed.). Pp.156176. Lecture Notes in Mathematics 363 . Springer Verlag, Berlin.

CIARLET, P.G. (1978) : "The Finite Element Method for Elliptic Problems ". Studies in Mathematics and its Applications Vol. 4. North-Holl and. Amsterdam.

CIARLET, P.G. (1982) : "Introduction à l'Analyse Numérique Matricielle et à 1 "Optimisation ", Masson, Paris.

CIARLET, P.G. ; DESTUYNDER, P. (1979a) : A justification of the two-dimensional linear plate model, J. Mecanique 18, 315-344.

CIARLET, P.G. ; DESTUYNDER, P. (1979b) : A justification of a nonlinear model in plate theory. Comp. Methods Appl. Mech. Engrg. 17. 227-258.

CIARLET, P.G. ; RAVIART, P.-A. (1972) : General Lagrange and Hermite Interpolotion in $R$ with opplications to finite element methods, Comput. Methods App 1. Mech. Engrg. 1 , 217-249.

CIARLET, P.G. ; RAVIART, P.-A. (1974): A mixed finite element method for the bihormonic equation, in "Mathematical Aspects of Finite Elements in Partial Differential Equations " (C.De Boor. Ed.). Pp.125-145, Academic Press. New York.

CIAVALDINI, J.F. ; NEDELEC, J.C. (1974): Sur l'élément de Fraeijs de Veubeke et Sander. R.A.I.R.O. Anal. Numer. R2, 29-45.

DAUTRAY, R. ; LIONS, J. L. (1984) ; Analyse Mathematique et Calcul Numérique pour les Sciences et les Techniques. Tome 1. Collection Commissariat à l'Energie Atomique. Masson. Paris.

DARLOW, B. ; EWING, R.E. ; WHEELER, M.F. (1984) : Mixed finite element method for miscible displacement problems in porous media, Soc. Pet. Eng. J. 24, pp. 391-398.

DAY, M.L. ; YANG, T.Y. (1982) : A mixed variational principle for finite element analysis, Internat. J. Numer. Meths. Engrg. 18. $1213-1230$.

DESTUYNDER, P. (1986) : Une Théorie Asymptotique des Plaques Minces en Elasticité Lineaire. Recherches en Mathématiques Appliquées 2. Masson, Paris.

DESTUYNDER, P. ; NEDELEC, J.-C. (1986) : Approximation numérique du cisailiement transverse duñs les plaques minces en flexion. Numer. Math. 48, 281-302.

DOUGLAS, J., Jr. (1962) : Alternating-direction methods for three space variables, Num. Math. 4, 41-63. 
DOUGLAS, J., Jr. (1984): Numerical methods for the flow of miscible fluids in porous media, in "Numerical Methods in Coupled Systems". (R.W. Lewis, P. Betess, E. Hinton), John Wiley \& Sons, Chichester.

DOUGLAS, J., Jr. ; DURAN, R. ; PIETRA, P. (1987a) : Formulation of alternating-direction iterative methods for mixed methods in three space, in Numerical Approximation of Partial Differential Equations (E. L. Ortiz, Ed.). Mathematics Studies 133, pp.21-30.

DOUGLAS, J., Jr. ; DURAN, R. ; PIETRA, P. (1987b) : Alternatingdirection iteration for mixed finite element methods, in "Computer Methods in Appl. Sci. and Engrg ", Vol. 7 (R. Glowinski, J.L. Lions, Eds.). North-Holl and, Amsterdam.

DOUGLAS, J., Jr. ; EWING, R.E. ; WHEELER, M.F. (1983): The approximation of the pressure by a mixed method in the simulation of miscible displacement, R.A.I.R.O. Anal. Numer. 17, 17-33.

DOUGLAS, J.. Jr. ; GUPTA, C.P. (1986) : Superconvergence for a mixed finite element method for elastic wave propagation in a plane domain. Numer. Math. $49,189-202$.

DOUGLAS, J., Jr. ; MILNER, A. (1985) : Interior and super convergence estimates for mixed methods for second order elliptic problems. M2AN $19,397-428$.

DOUGLAS, J.,Jr. ; PIETRA, P. (1986) : A description of some alternating-direction iterative techniques for mixed finite element methods, in "Mathematical and Computational Methods in Seismic Exploration and Reservoir Modeling" (W.E. Fitzgibbon, Ed.), pp. 37-53, SIAM. Philadelphia.

DOUGLAS, J., Jr. ; ROBERTS, J.E. (1982) : Mixed finite element methods for second order elliptic problems. Math. Apl. Comput. 1, 91-103.

DOUGLAS, J., Jr. ; ROBERTS, J.E. (1983) : Numerical methods for a model for compressible miscible displacement in porous media, Math. Comp. 41 , 441-459.

DOUGLAS, J., Jr. ; ROBERTS, J.E. (1985) : Global estimates for mixed methods for second order elliptic problems. Math. Comput. $44,39-52$.

DUVAUT, G. ; LIONS, J.-L. (1972): "Les Inéquations en Méconique et en Physique ", Dunod, Paris.

EWING, R.E. ; WHEELER, M.F. (1983) : Computational aspects of mixed finite element methods, in "Scientific Computing" ( $R$. Stepleman et al. Eds.), pp. 163-172, IMACS/North Holl and, Amsterdam.

EYMARD, R. ; GALLOUET, T. ; JOLY, P. (1987) : Ecoulement d'un fluide diphosique incompressible dans un milieu poreux en dimension 2 ou 3 , to appear.

FALK, R.S. (1976): A Ritz method based on complementary variational principle, R.A.I.R.O. Anal. Numér. 10,39-48.

FALK, R.S. (1978) : Approximation of the biharmonic equation by a mixed finite element method, SIAM J. Numer. Anal. 15,556567. 
FALK, R.S. ; OSBORN J.E. (1980) : Error estimates for mixed methods. R.A.I.R.O. Anal. Numér. 14, 249-277.

FELIPPA, C.A. (1986) : Penalty-function iterative procedures for mixed finite element formulations, Internat. J. Numer. Meths. Engrg. 22 , 267-279.

FIX, G.J. (1976) : Hybrid element methods, SIAM Rev. 18, 460-484.

FIX, G.J. ; GUNZBERGER, M.D. ; NICOLAIDES, R.A. (1979) : On finite element methods of least squares type. Comput. Methods App 1. Mech. Engrg. 10,175-198.

FIX, G.J. ; GUNZBERGER, M.D. ; NICOLAIDES, R.A. (1981) : On mixed finite element methods for first order ellptic systems, Numer. Math. $37,29-48$.

FORTIN, M. (1977) : Analysis of the convergence of mixed finite element methods, R.A.I.R.O. Anal. Numer. 11, R $4,341-354$.

FORTIN, M. (1985) : A three-dimensional quadratic nonconforming element. Numer. Math. $46,269-279$.

FORTIN, M. ; GLOWINSKI, R. (1983) : "Résolution de problèmes aux limites par des méthodes de Lagrangien augmenté". Dunod, Paris.

FORTIN, M. ; GLOWINSKI, R. (1983) : "Augmented Lagrangian Methods. Application to the Numerical Solution of the Boundary Value Problems ". Studies in Mathematics and its Applications Vol. 15, North-Holl and, Amsterdam.

FRAEIJS DE VEUBEKE, B. (1965) : Displacement and equilibrium models in the finite element method, Chapter 9 of "Stress Analysis " (0.C. Zienkiewicz, \& G.S. Holister, Eds.). pp.145-197. Wiley, New York.

FRAEIJS DE VEUBEKE, B. (1973) : Diffusive equilibrium models, University of Calgary Lecture Notes, Internat. Research Seminar on the Theory and Applications of the Finite Element Methods, Calgary.

FRAEIJS DE VEUBEKE, B. (1974a) : Finite element method in aerospace engineering problems in Computing Methods in Appl. Sci. and Engrg. (R. Glowinski \& J. L. Lions, Eds.), Lecture Notes on Computer Science, Vol.16, pp.224-258, Springer, Berlin.

FRAEIJS DE VEUBEKE, B. (1974b) : Variational principles and the patch test. Internat. J. Numer. Meths. Engrg. 8. 783-801.

FRAEIJS DE VEUBEKE, B. (1975) : Stress Function Approach, in "World Congress on Finite Element Methods in Structural Mechanics". Vol.1. pp.J.1-J.51, Bournmouth, Dorset. England.

FRAEIJS DE VEUBEKE, B. ; HOGGE, M.A. (1972): Dual analysis for heat conduction problems by finite elements, Internat. J. Numer. Meths. Engrg. 5, $65=82$.

FRAEIJS DE VEUBEKE, B. ; SANDER, G. (1968): An equilibrium model for plate bending. Internat. J. of Solids and Structures 4. $447-468$. 
GASTALDI, L. ; NOCHETTO, R. (1987) : Optimal L astimates for nonconforming and mixed finite element methods of lowest order. Numer. Math. 50, 587-611.

GIRAULT, V. ; RAVIART, P.-A. (1979) : "Finite Element Approximation of the Navier-Stokes Equations ", Lecture Notes in Mathematics 749 , Springer-Verlag. Berl in Heidelberg.

GIRAULT, V. ; RAVIART, P.-A. (1986) : "Finite Element Methods for Navier-Stokes Equations. Theory and Algorithms ". Lecture Notes in Computational Mathematics 5, Springer-Verlag. Berlin.

HASLINGER, J. ; HLAVACEK, I. (1975) : Curved element in a mixed finite element method, Applikace Matematiky 20 , 233-252.

HASLINGER, J. ; HLAVACEK, I. (1976a) : Convergence of on equilibrium finite element method based on the dual variational formulation. Applikace Matematiky $21,43-65$.

HASLINGER, J. ; HLAVACEK, I. (1976b) : A mixed finite element method close to the equilibrium model, Numer. Math. 26, 85-97.

HASLINGER, J. ; NEITTAANMAKI, P. (1984): On different finite element methods for approximating the gradient of the solution to the Helmholtz equation, Comput. Meths. Appl. Mech. Engrg. $42,131-148$.

HECHT F. (1981) : Construction d'une base d'un element fini $P$ non conforme ò divergence nulle dans $R$. RAIRo Anal. Numér. 15, 119-150.

HELLAN, K. (1967) : Analysis of elastic plates in flexure by a simplified finite element method, Acta Polytechnica Scandi navia, Civil Engineering Series 46. Trondheim.

HENNART, J.P. (1985) : Nodal schemes, mixed-hybrid finite elements and block-centered finite differences, Rapport de Recherche INRIA 386, Rocquencourt.

HENNART, J.P. (1986a) : A general family of nodal schemes, SIAM J. Sci. Stat. Comput. 3. 264-287.

HENNART, J.P. (1986b) : A general finite element framework for nodal methods, in "MAFELAP 1984, The Mathematics of Finite Elements and Applications "( J.R. Whiteman , Ed.),pp.309-316. Academic Press, London.

HENNART, J.P. ; JAFFRE, J ; ROBERTS, J.E. (1986) : A constructive method for deriving finite elements of nodal type, Rapport de Recherche INRIA 541, Rocquencourt.

HERRMANN, L.R. (1967) : Finite element bending analysis for plates, J. Eng. Mech. Div.,ASCE 93, No EM5, 13-26.

HERRMANN, L.R. (1983) : Mixed finite elements for couplestress analysis, in "Hybrid and Mixed Finite Element Methods "( S.N. Atlury, R.H. Gallagher, \& O.C. Zienkiewicz, Eds.), pp.1-17, John Wiley \& Sons, Chichester.

HLAVACEK, I. (1979) : Convergence of on equilibrium finite 

427-457.

HLAVACEK, I. (1980) : The density of solenoidal functions and the convergence of a dual finite element methods, Applikace Matematiky $25,39-55$.

HLAVACEK, I. ; KRIZEK, M. (1984) : Internal finite element approximations in the dual variational methods for second order elliptic problems with curved boundaries, Applikace Matematiky 29, 52-69.

IRONS, B.M. (1972) : An assumed-stress version of the Wilson 8-Nodes isoparametric brick. Computer Report CNME/56/. University of Wales. Swansea.

IRONS, B.M. (1984) : Alternative ways for the formulation of hybrid stress elements, Internat. J. Numer. Meths. Engrg. 20. 780-782.

IRONS, B.M. ; LOIKKANEN, M. (1983) : An engineers'defence of the patch test, Internat. J. Numer. Meths. Engrg. 19, 1391-1401.

IRONS, B.M. ; RAZZAQUE, A. (1972) : Experience with the patch test for convergence of finite elements, in "The Mathematical Foundations of the Finite Element Method with Applications to Partial Differential Equations "( A.K. Aziz. Ed.). pp.557-587, Acodemic Press, New York.

JAFFRE, J. (1984) : Elements finis mixtes et decentrage pour les equations de diffusion-convection, Calcolo $23,171-197$.

JAFFRE, J. ; ROBERTS, J.E. (1985) : Upstream weighting and mixed finite elements in the simulation of miscible displacements. M2AN $19,443-460$.

JIROUSEK, J. ; LAN GUEX (1986) : The hybrid-Trefftz finite element model and its application to plate bending. Internat. J. Numer. Meths. Engrg. 23, 651-693.

JOHNSON, C. (1973) : On the convergence of the mixed finite element method for plate bending problems. Numer. Math. $21,43-$ 62.

JOHNSON, C. ; MERCIER, B. (1978) : Some equilibrium finite element methods for two-dimensional elasticity problems. Numer. Math. $30,103-116$.

JOHNSON, C. ; MERCIER, B. (1979) : Some equilibrium finite element methods for two-dimensional problems in continuum mecha nics, in "Energy Methods in Finite Element Analysis "( R. Glowinski. E.Y. Rodin, \& O.C. Zienkiewicz, Eds.), pp.213-224, John Wiley \& Sons, Chichester.

JOHNSON, C. ; PITKARANTA, J. (1982): Analysis of some finite element methods related to reduced integration, iath. Comput. $38,375-400$.

JOHNSON, C. ; THOMEE, V. (1981) : Error estimates for some mixed element methods for parabolic type problems, R.A.I.R.O. Anal. Numer. 15, 41-78. 
JOLY, P. (1982) : La methode des élements finis mixtes appliquee au problème de diffusion-convection. Thèse de 3eme cycle, Université P. \& M. Curie, Paris.

JOLY, P. (1984) : Résolution de systèmes linéaires non symetriques par des méthodes de gradient conjugué. Publications du Laboratoire d'Analyse Numerique. Université Paris 6.

JOLY, P. (1986) : Presentation de synthèse des méthodes de gradient conjugue. M2AN 20,639-665.

KIKUCHI, F. (1973) : Some considerations of the convergence of hybrid stress method, in "Theory and Pratice in Finite Element Structural Analysis "( $Y$. Yamada \& R.H. Gallagher, Eds.), PP.2542. University of Tokyo Press.

KIKUCHI, F. (1983) : On a mixed method related to the discrete Kirchhoff assumption, in "Hybrid and Mixed Finite Element Methods "( S.N. Atlury, R.H. Gallagher, \& O.C. Zienkiewicz, Eds.), Pp.137-154, John Wiley \& Sons, Chichester.

KRIZEK, M. (1982): An equilibrium finite element method in three-dimensional elasticity. Applikace Matematiky 27, 46-75.

KRIZEK, M. (1983) : Conforming equilibrium finite element methods for some elliptic plane problems, R.A.I.R.O. Anal. Numer. 17 . 35-65.

KRIZEK, M. ; NEITTAANMAKI, P. (1986) : Internal FE approximation of spaces of divergence-free functions in three-dimensional domains, Int. J. Numer. Methods Fluids 6, 811-817.

LADYZHENSKAYA, O.-A. (1969): "The Mathematical Theory of Viscous Flow", (English Translation of Russian, 2nd Edition). Gordon and Breach, New York.

LASCAUX, P. ; LESAINT, P. (1973) : Conforming and non conforming finite elements for the plate bending problems, R.A.I.R.O. Anal. Numér. $1,9-53$.

LEE, S.W. ; RHIU, J.J. (1986): A new approach to the formulation of mixed finite element models for structural onalysis, Internat. J. Numer. Meths. Engrg. $21,1629-1641$.

LEE, S.W. ; WONG, S.C. ; RHIU, J.J. (1985) : Study of a nine node mixed formulation finite element for thin plates and shells, Computers and Structures $21,1325-1334$.

LE ROUX, M.-N. (1982) : A mixed finite element method for a weighted elliptic problem, R.A.I.R.O. Anal. Numer. $16,243-273$.

LESAINT, P. (1976) : On the convergence of Wilson's non conforming element for solving the elastic problem, Comput. Methods App 1. Mech. Engrg. $7,1-6$.

LESAINT, P. ; ZLAMAL, $M$. (1980) : Convergence of the non conforming Wilson element for arbitrary quadrilateral meshes, Numer. Math. $36,33-52$.

LI, Z.C. ; LIANG. G.P. (1981) : On the simplified hybrid- 
combined method, Math. Comput. 41, 13-25.

LIONS, J. L. ; MAGENES, E. (1968) : "Problemes aux Limites non Homogenes et Applications". Vol. 1, Dunod Paris

MALKUS, D.S. ; HUGHES, T.J.R. (1978) : Mixed finite element methods. reduced and selective integration techniques: A unification of concepts, Comput. Methods Appl. Mech. Engrg. $15,63-$ 81 .

MARINI, L.D. (1983) : An inexpensive method for the evaluation of the solution of the lowest order Raviart-Thomas mixed method, SIAM J. Numer. Anal. $22,493-496$.

MARSDEN, J.E. \& HUGHES, T.J.R. (1983) : "Mathematical Foundations of Elasticity ", Prentice-Hall, Inc., Englewood Cliffs, New Jersey.

MERCIER, B. (1974): Numerical solution of the biharmonic problem by mixed finite elements of class $C O$, Bull. Un. Mat. Ital. $10,133-149$.

MERCIER, B. ; OSBORN, J. ; RAPPAZ, J. ; RAVIART, P.-A. (1981) : Eigenvalue approximation by mixed and hybrid methods, Math. Comput. $36,427-453$.

MIGNOT, A.L. ; SURRY, C. (1981) : A mixed finite element family in plane elasticity. Appl. Math. Modelling 5, 259-262.

MILNER, F. (1985) : Mixed finite element methods for quasilinear second order elliptic problems, Math. Comput. 44, 303-320.

MIRZA, F.A. ; OLSON, M.D. (1980): The mixed finite element method in plane elasticity. Internat. J. Numer. Meths. Engrg. 15. 273-289.

MIYOSHI, T. (1973) : A finite element method for the solution of fourth order partial differential equations, Kumamoto J. Scl. (Math.) $9,87-116$.

MIZUKAMI, A. (1986) : A mixed finite element method for boundary flux computation, Comput. Meths. Appl. Mech. Engrg. 57. 239-243.

MIZUKAMI, A. ; HUGHES, T.J.R. (1985) : A Petrov-Galerkin finite element method for convection-dominoted flows: an accurate upwinding technique for satisfying the maximum principle, Comput. Meths. Appl. Mech. Engrg. 50, 181-193.

MORLEY, L.S.D. (1968) : The triangular equilibrium element in the solution of the plate bending problems, Aero. Quart. 19. 255-269.

NEDELEC, J.C. (1980) : Mixed finite elements in R3, Numer. Math. $35,315-341$.

NEDELEC, J.C. (1986) : A new family of mixed finite element in R3, Numer. Math. 50, 57-81.

NEITTAANMAKI, P. ; SARANEN, J. (1981): On the finite element approximation of gradient for solution of Poisson equation. 
Numer. Math. $37,333-337$.

NICOLAIDES, R. A. (1982) : Existence, uniqueness and approximation for generalized saddle point problems, SIAM J. Numer. Anal. 19. 349-357.

NOOR, A.K. ; PETERS, J.M. (1983) : Mixed models and reduced selective integration displacement models for vibration analysis of shells, in "Hybrid and Mixed Finite Element Methods " (S.N. Atlury, R.H. Gallagher, \& O.C. Zienkiewicz, Eds.), pp.537-564, John Wiley \& Sons, Chichester.

ODEN, J.T. (1973) : Some contributions to the mathematical theory of mixed finite element approximations, in "Theory and Pratice in Finite Element Structural Analysis "( $Y$. Yamado \& R.H. Gallagher, Eds.), Pp.3-23, University of Tokyo Press.

ODEN, J.T. ; LEE, J.K. (1975) : Theory of mixed and hybrid finite element approximations in linear elasticity. in "Proc. "IUTAM ". IUM Symp. Applications of Methods of Functional Analysis to Problems of Mechanics, Marseille. Sept. 75.

ODEN, J.T. ; REDOY, J.N. (1975): Some observations on properties of certain mixed finite element approximations, Internat. J. Numer. Meths. Engrg. $9,933-949$.

ODEN, J.T. ; REDDY, J.N. (1976a) : On mixed finite element approximations, SIAM J. Numer. Anal. $13,392-404$.

ODEN, J.T. : REDDY, J.N. (1976b) : "An Introduction to the Mathematical Theory of the Finite Elements" . John Wiley \& Sons, New York.

PEACEMAN, D.W. ; RACHFORD H.H., Jr. (1955) : The numerical solution of parabolic and elliptic differential equations, J. Soc. Ind. Appl. Math. 3, 28-41.

PIAN, T.H.H. (1964) : Derivation of element stiffness matrices by ossumed stress distributions, A.I.A.A. J. 2, 13331336.

PIAN, T.H.H. (1971): Formulations of finite element methods for solid continua, in "Recent Advances in Matrix Methods of Structural Analysis and Design "( R.H. Gallagher, Y. Yamada, \& J.T. Oden, Eds.), Pp.49-83, The University of Alabama Press.

PIAN, T.H.H. (1972) : Finite element formulation by variational principles with relaxed continuity requirements, in "The Mathematical Foundations of the Finite Element Method with Applications to Partial Differential Equations "( A.K. Aziz, Ed.), pp.671-687. Academic Press, New York.

PIAN, T.H.H. (1982): On the equivalence between incompatible displacement element and hybrid stress element. Appl. Math. Mech. (English Edition). Chung King, China, $3(6), 773-776$.

PIAN. T.H.H. (1983) : Reflections and remarks on hybrid and mixed finite element methods, in "Hybrid and Mixed Finite Element Methods" (S.N. Atlury, R.H. Gallagher. \& O.C. Zienkiewicz, Eds.), mn 565-570. John Wiley \& Sons, Chichester. 
PIAN T.H.H. $(1985)$ assumed stresses and displacements. and Design $1,131-140$.

lement based on consistently Finite Elements in Analysis

PIAN, T.H.H. : CHEN, D.P

formulation of hybrid stress ement Engrg. $18,1676-1684$.

PIAN, T.H.H. : CHEN, D.P. ; KANG, D. (1983) : A new formulation of hybrid-mixed finite elements, Comput. \& Structures $16,81-87$.

PIAN, T.H.H. ; KANG, D. ; WANG, C. (1987) : Hybrid plate elements based on balanced stresses and displacements, to be published in "State-of-the-Art Texts on Finite Element Methods in Plate and Shell Structural Analysis "( T.J.R. Hughes \& E. Hinton Eds.).

PIAN, T.H.H. ; LI, M.-S. ; KANG, D. (1986) : Hybrid stress elements based on natural isoparametric coordinates, in "Proceedings of the Invitational China-American Workshop on Finite "Element Methods" . Chengde, People's Republic of China, June 2-6 1986.

PIAN, T.H.H. ; SUMIHARA, K. (1984) : Rational approach for assumed stress finite elements, Internat. J. Numer. Meths. Engrg. 22 . 173-181.

PIAN, T.H.H. ; TONG, P. (1969) : Basis of finite element methods for solid cont inua. Internat. J. Numer. Methods. Engrg. 1 , 3-28.

PIAN, T.H.H. ; TONG, P. (1986) : Relations between incompatible displacement model and hybrid stress model, Internat. J. Numer. Meths. Engrg. $22,173-181$.

PITKARANTA, J. (1979) : Boundary subspaces for the finite elements methods with Lagrange multipliers. Numer. Math. $33,273-$ 289.

PITKARANTA, J. (1980a) : Local stability conditions for the Babuska method of Lagrange multipliers, Math. Comput. 35, 11131129 .

PITKARANTA, J. (1980b) : A conforming finite element method with Lagrange multipliers for the biharmonic problem, R.A.I.R.O. Anal. Numer. $14,309-324$.

PITKARANTA, J. (1981) : The finite element method with Lagrange multipliers for domains with corners, Math. Comput. 37 . $13-30$.

PITKARANTA, J. : STENBERG, R. (1983): Analysis of some mixed finite element methods for plane elasticity equations, Math. Comput. $41,399-423$.

POWELL M.J.D. (1969) : A method for nonlinear constraints in minimization problems, in "Optimization "( $R$. Fletcher Ed.). pp. 283-298, Acodemic Press, London.

PUNCH, E.F. ; ATLURI, S.N. (1984) : Development and testing of stable, invariant, isoparametric curvilinear 2-and 3-D hybrid- 
THOMEE, $\quad V . \quad(1984)$ : "Galerkin Finite Element Methods for "Porabolic Problems". Lecture Notes in Mathematics 1054 . SpringerVerlag, Berlin.

TIAN, Z.S. ; PIAN, T.H.H. (1985) : Axisymetric solid elements by a' rational hybrid stress method. Computers and Structures $20,141-149$.

TONG, P. (1969) : An assumed stress hybrid finite element method for incompressible materials, Internat. J. Solids and Structures $5,455-461$.

TONG, P. (1982) : A family of hybrid plate elements, Internat. J. Numer. Meths. Engrg. 15, 1771-1812.

TONG, P. ; PIAN, T.H.H. (1969) : A variational principle and the convergence on a finite element method based on assumed stress distribution. Internat. J. Solids Struct. $5,463-472$.

WHEELER, M. ; GLOWINSKI. R. (1987) : Domain decomposition for mixed finite element methods. presented at the First World Congress on Computational Mechanics, Austin. Texas, Sept. 1986, to appear.

WHEELER, M. ; GONZALEZ, R. (1984) : Mixed finite element methods for petroleum reservoir engineering problems, in "Computing Methods in Applied Sciences and Engineering. VI" (R. Glowinski \& J.-L. Lions, Eds.). pp. 639-657, North Holl and, Amsterdam.

WILSON, E.L. ; TAYLOR, R.L. ; DOHERTY, W.P. ; GHABOUSSI, J. (1973) : Incompatible displacement models, in "Numerical and Computer Methods in Structural Mechanics" (S.J. Fenves et al. Eds.). pp.43-57. Academic Press. New York.

WOLF, J.P. (1972a) : Structural averaging of stresses in the hybrid stress model. A.I.A.A. J. $10,843-845$.

WOLF, J.P. (1972b) : Generalized hybrid stress finite element models, A.I.A.A. J. $11,386-388$.

ZHONG-CI SHI , (1984a) : On the convergence properties of the quadrilateral elements of Sander and Beckers, Math. Comput. 42. 493-504.

ZHONG-CI SHI, (1984b) : A convergence condition for the quadrilateral Wilson element. Numer. Math. 44, 349-361.

ZHONG-CI SHI, (1984c) : An explicit analysis of the Stumel's patch' test examples, Internat. J. Numer. Meths. Engrg. $20,1233-1246$.

ZIELINSKI, A.P. ; ZIENKIEWICZ O.C. (1985) : Generalized finite element analysis with T-complete boundary solutions functions, Internat. J. Numer. Meths Engrg. $21,509-528$.

ZIENKIEWICZ, O.C. ; LI XI-KUI ; NAKASAWA, S. (1984) : Iterative solution of mixed problems and the stress recovery procedures, Research Report C/R476/84, Inst. for Numerical Methods in Engineering. University College of Swansea. Wales, U.K. 
element methods,. in "First World Congress on Computational "Mechanics ".Austin. Texas. Sept. 1986, to appear.

STEIN, E. : AHMAD, R. (1977) : An equilibrium method for stress calculation using finite element displacement models. Comput. Methods App 1. Mech. Engrg. 10, 175-198.

STENBERG, R. (1986) : On the construction of optimal mixed finite element methods for the linear elasticity problem, Numer. Math. $48,447-462$.

STOLARSKI, H. ; BELYTSCHOKO, T. (1986a) : On the equivalence of mode decomposition and mixed finite elements based on the HellingerReissner principle. Part I: Theory, Comput. Methods Appl. Mech. Engrg, 249-263.

STOLARSKI, H. ; BELYTSCHOKO, T. (1986b) : On the equivalence of mode decomposition and mixed finite elements based on the HellingerRe issner principle, Part II: Applications, Comput. Methods Appl. Mech. Engrg 58, 265-284.

STRANG, G. (1972) : Variational crimes in the finite element method, in "The Mathematical Foundations of the Finite Element Method with Applications to Partial Differential Equations" ( A. K. Aziz, Ed.). pp. 689-710. Academic Press, New York.

STRANG, G. ; FIX, G.J. (1973) : "An Analysis of the Finite Element Method". Prentice-Hall, Englewood Cliffs, New Jersey.

STUMMEL, F. (1979): The generalized patch test, SIAM J. Numer. Anal. $16,449-471$.

STUMMEL, F. (1980a) : The limitations of the patch test, Internat. J. Numer. Meths. Engrg. $15,177-188$.

STUMMEL, F. (1980b) : Basic compactness properties of non conforming and hybrid finite element spaces, R.A.I.R.O. Anal. Numér. $4,81-115$.

TAYLOR, R.L. ; BERESFORD, P.J. ; WILSON, E.L. (1976) : A non conforming element for stress analysis, Internat. J. Numer. eths. Engrg. 10 , 1211-1219.

TEMAM, R. (1979) : "Navier-Stokes Equations ", Studies in Mothematics and its Applications Vol. 2, North-Holland, Amsterdam.

THOMAS, J.-M. (1976) : Methode des elements finis hybrides duaux pour les problemes du second ordre, R.A.I.R.O.. Anal. Numér. 10 , No.12, 51-79.

THOMAS, J.-M. (1977) :" Sur l'Analyse Numérique des Méthodes "d'Eléments Finis Hybrides et Mixtes". These d'Etat, Université Pierre et Marie Curie. Paris.

THOMAS, J.-M. (19870) : Mixed finite elements methods for convection-diffiusion problems, in "Numerical Approximation of Portial Differential Equations". (E. L. Ortiz, Ed.). Mathematics Studies 133. Pp. 241-250, North-Hol land, Amsterdam.

THOMAS, J. - M. (1987b) : On the convergence of mixed finite elements methods, presented at the I.C.I.A.M. 87. Paris-La Villette. 
ZIENKIEWICZ, O.C. ; QU, S. ; TAYLOR, R.L. ;NAKAZAWA, S. (1986)

The patch test for mixed formulations, Internat. J. Numer. Meths.

Engrg. 23, 1873-1883. 
(3) 